\title{
EXAMINATION OF MICROVASCULAR REMODELING TO ENHANCE AUTOLOGOUS FAT GRAFTING
}

\author{
A Dissertation \\ Presented to the faculty of the School of \\ Engineering and Applied Science in partial \\ fulfillment of the requirements for the degree of \\ Doctor of Philosophy
}

\section{by ScOTT Alan SEAMAN}

May 2016

DEPARTMENT OF BIOMEDICAL ENGINEERING

UNIVERSITY of VIRGINIA 


\section{APPROVAL SHEET}

This dissertation is in partial fulfillment of the requirements for the degree of Doctor of Philosophy in Biomedical Engineering

Scott Alan Seaman

Author

This dissertation has been read and approved by the examining committee:

Shayn Peirce-Cottler, Ph.D

Dissertation Advisor

Department of Biomedical Engineering

Song Hu, Ph.D

Committee Chair

Department of Biomedical Engineering

Chris Campbell, M.D.

Committee Member

Department of Plastic Surgery

Patrick Cottler, Ph.D.

Committee Member

Department of Plastic Surgery

Norbert Leitinger, Ph.D.

Committee Member

Department of Pharmacology

Accepted for the School of Engineering and Applied Science:

Craig H. Benson, Ph.D.

Dean

School of Engineering and Applied Science 


\section{DEDICATION}

First and foremost, I'd like to thank my research advisor, my mentor, and my friend, Dr. Shayn Peirce-Cottler. Shayn - your passion, optimism, and enthusiasm for science is pervasive and has inspired me to strive to become as good of a scientist, mentor, and leader as you are. I remember countless meetings where I felt discouraged having reported no new data or progress, but our brainstorming sessions (often $2+$ hours) made me excited to get back to work and somehow always made me feel positive about my projects. This positive energy that you bring to your trainees, colleagues, and collaborators is something that I wish to emulate in the future. You are directly responsible for my professional, academic, and personal development and I am forever indebted to you for that. Thank you so much for being the best advisor and mentor I could have asked for and thank you for being a true friend during my graduate school career. I've learned by your great example, Shayn, and am excited to go share it with the rest of the world.

During my time here at the University of Virginia, it has been a pleasure to work with and form collaborations with so many great people. Their encouragement and support is really remarkable and has made the trip through graduate school that much more fulfilling. I would like to thank my thesis committee - Dr. Chris Campbell, Dr. Patrick Cottler, Dr. Song Hu, and Dr. Norbert Leitinger - for all of your feedback and advice. To Dr. Campbell, I appreciate you teaching me the correct plastic surgery vernacular and for helping me become more clinically aware. To Patrick, thank you for helping coordinate many of my experiments with the busy UVa plastic surgery team and for always being able to crack a joke or put a smile on my face. To Song, thank you for encouraging me to think about different ways to image blood vessels, for being a collaborator, and for serving as the chair of my committee. To Norbert, I appreciate you lending me some of your immunology expertise and for helping shape many of my studies focused on macrophages. You all have pushed me past my comfort zone and forced me to become a better scientist because of it. Thank you all for serving on my committee. The UVa Department of Plastic Surgery has been a source of many collaborations, advice, and resources and I would like to thank my past collaborators and coauthors for their help and support - Dr. Shruti Tannan, Dr. Kant Lin, Dr. Thomas Gampper, Dr. Bryce Olenczak, Dr. 
Angela Pineros-Fernandez, and Lisa Salopek. I would also like to thank Dr. Cassandra Fraser and Chris DeRosa in the UVa Department of Chemistry for allowing me to be a part of the development of an exciting clinical diagnostic device.

To the past and present Peirce Pirates/members of the lab, it has been a pleasure working with all of you. I'll miss experiencing the joys (and difficulties) of research, our coffee runs, Last Thursday happy hours, croquet matches, our shared commiserating, and bocce tournaments, but I look forward to our continued friendships. To Bryan, Jason, and Tom, thank you for showing me the ropes and getting me started when I was a new graduate student. Alongside me throughout my graduate work - Joe, Kyle, and Anthony - thank you for being great friends, coworkers, and scientists to help refine my work. Anthony, you deserve a special thank you for keeping the lab running smoothly even when we tried to veer off course. To Molly, Bruce, and Clif, I appreciate the help that you all have provided and hope that I have reciprocated in some way to your work and look forward to seeing all of your successes in the future. To the undergraduates that worked directly with me - Yiqi, Ethan, and Cat - thank you for helping with experiments, analyzing data, and for teaching me about the UVa culture. I'm very fortunate to work with all of these talented individuals and am excited for our future friendships.

Thank you to all of my fellow graduate students, their significant others, and my other Charlottesville friends that have been a part of many fun and exciting adventures along the way. I've forged a new set of friends here in Charlottesville (way too long to list) and I am looking forward to our continued relationships. I would especially like to thank Eric, Sameer, and Edik for their continued friendship and encouragement. Eric, thanks for teaching me everything there is to know about beer and for explaining the game of rugby to me (maybe one day I'll understand it). Sameer, thank you for always being willing to talk about sports and, in general, arguing (discussing?) Georgia Tech and UVa sports. Edik, thanks for being so positive about everything and for your happy-go-lucky/West coast attitude that brought a fresh perspective to my life. I am truly lucky to develop and maintain so many great relationships during graduate school.

My family deserves much of the credit for my successes. Mom and Dad, thank you for always pushing me to try my hardest and for enabling me to journey down 
this privileged path. While my parents don't know exactly what I do, they have never stopped telling me how proud they are of me and have always been my number one supporter. To answer your recurring question Mom and Dad, "school is going well". I love you, Mom and Dad. To my brother/role model/best friend Marc, thank you for setting such a great standard for me to live up to. I've followed your example from when we were young and will continue to look up to you. To Amanda, I also want to thank you for your support over the years. I also owe gratitude to extended family members that have supported me along the way - Aunt Nancy, AK and UA, the Drewiczs, the Ewings, the Centofantis, and the Swansons. To all of my friends and family that are not explicitly listed, thank you all for your love and support.

And finally, to my wife, Stephanie. Thank you for your unwavering love, support, and motivation during this chapter of our lives. You uprooted your life, trusted me, and displayed patience all for me to chase a dream and I can't thank you enough for that. I'm so grateful that you have been here to support me and I look forward to a new chapter in our lives. I love you.

- Scott

February 2016

Charlottesville, VA

This work was made possible by the generosity of the University of Virginia, the Biotechnology Training Program, National Institutes of Health Funding, and the Hartwell Foundation. 


\section{ABstract}

There exists a clinical need for repairing soft tissue defects resulting from pathological conditions, trauma, congenital disorders, and post-operative cancer resections. Autologous fat has been regarded as an appealing tissue for these surgeries due to its innate biocompatibility, high degree of vascularity, and low risk for immune rejection. Indeed, autologous fat grafting in plastic and reconstructive surgery is increasing and typically manifests in two different practices/scenarios: (i.) fat grafting, which typically involves non-surgical injection of micro-volumes of fat, and (ii.) flap transfer, which involves surgical composite tissue transfer of macrovolumes of fat, muscle, and often skin. Despite the popularity of using autologous fat in reconstructive surgery, graft survival and long-term volume retention is highly variable and unpredictable. Further, resorption rates of grafted fat ranges from $25-80 \%$ strengthening the need for investigation into fat graft survival. This resorption process is poorly understood and the underlying mechanism(s) responsible for long-term volume retention of fat grafts remains to be elucidated. I submit that fat engraftment and resorption are dynamic processes resulting from the intersection of key mechanisms, including adipocyte adaptations, vascular remodeling, and inflammation. Using a comprehensive and integrative strategy, my dissertation evaluates these three mechanisms in culture systems and in different murine models. I develop and employ novel imaging strategies, transgenic mice, and analysis metrics, with the ultimate goal of designing therapeutic interventions for enhancing adipose tissue regeneration and graft survival. Toward this end, my research makes four key contributions: (i) determining how vascular network remodeling in flaps (macro-grafts) and fat grafts (micro-grafts) both affect and are affected by perfusion and inflammation, (ii) identifying macrophages as a novel drug target and evaluating a new pharmacological treatment for improving longterm survival of fat grafts, (iii) evaluating and optimizing enzymatic digestion and stromal vascular fraction enhancement for micro-graft volume retention and adipocyte viability, and (iv) validating a novel imaging approach for monitoring oxygen perfusion in tissues. Together, my insights and innovations substantially

advance the nascent field of adipose tissue engineering and motivate new tools and approaches that could have significant clinical impact. 


\section{CONTENTS}

DEDICATION

$\begin{array}{lll}\text { CONTENTS } & \text { VII }\end{array}$

\begin{tabular}{ll|l} 
LIST OF FIGURES & IX
\end{tabular}

\section{Chapter 1}

INTRODUCTION

Background 2

Autologous fat grafting $\quad 3$

$\begin{array}{lr}\text { Microvascular system } & 9\end{array}$

Adipose tissue anatomy and microstructure $\quad 16$

$\begin{array}{ll}\text { Monocytes and macrophages } & 18\end{array}$

Overview of thesis $\quad 22$

Chapter 2

MACROPHAGE RECRUITMENT AND POLARIZATION DURING COLLATERAL VESSEL REMODELING IN MURINE ADIPOSE TISSUE $\quad 29$

$\begin{array}{ll}\text { Abstract } & 30\end{array}$

Introduction $\quad 31$

Materials and methods $\quad 34$

Results $\quad 45$

Discussion $\quad 54$

$\begin{array}{ll}\text { Conclusions } & 62\end{array}$

CHAPTER 3

COLLATERAL VESSEL REMODELING IN MURINE ADIPOSE TISSUE IS CONTINGENT ON MACROPHAGE POPULATIONS

$\begin{array}{ll}\text { Abstract } & 64\end{array}$

Introduction $\quad 66$

Materials and methods $\quad 70$

Results $\quad 80$

Discussion $\quad 92$

$\begin{array}{ll}\text { Conclusions } & 102\end{array}$

\section{ChAPTER 4}

DIFFERENTIAL EFFECTS OF PROCESSING TIME AND DURATION OF COLLAGENASE DIGESTION ON HUMAN AND MURINE FAT GRAFTS $\quad 103$

$\begin{array}{ll}\text { Abstract } & 104\end{array}$

$\begin{array}{lr}\text { Introduction } & 106\end{array}$ 
Materials and methods $\quad 109$

$\begin{array}{ll}\text { Results } & 117\end{array}$

Discussion $\quad 121$

$\begin{array}{lr}\text { Conclusions } & 128\end{array}$

Chapter 5

EFFECTS OF COLLAGENASE DIGESTION AND SVF SUPPLEMENTATION ON VOLUME RETENTION OF FAT GRAFTS $\quad 129$

$\begin{array}{lr}\text { Abstract } & 130\end{array}$

Introduction $\quad 132$

Materials and Methods 136

Results

Discussion $r 4$

$\begin{array}{lr}\text { Conclusions } & 155\end{array}$

Chapter 6

PARADOXICAL ADIPOSE HYPERPLASIA AND CELLULAR EFFECTS AFTER CRYOLIPOLYSIS: A CASE REPORT $\quad 156$

Abstract $\quad 157$

Introduction $\quad 159$

Report of the case $\quad 162$

Discussion $\quad 169$

Conclusions 174

Chapter 7

USING DUAL-EMISSIVE OXYGEN-SENSITIVE PLA NANOPARTICLES TO MONITOR LOCAL WOUND OXYGENATION $\quad 175$

$\begin{array}{ll}\text { Abstract } & 176\end{array}$

Introduction $\quad 178$

Materials and Methods 182

$\begin{array}{lr}\text { Results } & 188\end{array}$

Discussion $\quad 194$

$\begin{array}{ll}\text { Conclusions } & 199\end{array}$

Chapter 8

DisCUSSION AND FUTURE DIRECTIONS $\quad 200$

Overview 201

History of reconstructive surgery $\quad 202$

Contributions and extended applications 204

Future Directions $\quad 210$

$\begin{array}{ll}\text { Final remarks } & 219\end{array}$

$\begin{array}{lc}\text { REFERENCES } & 221\end{array}$ 


\section{LIST OF FIGURES}

Figure 1.1: Micro-graft (fat graft) vs. macro-graft (flap) $\quad 6$

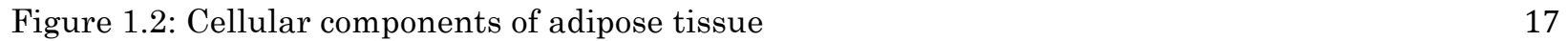

Figure 2.1: In vivo murine model of flap delay $\quad 35$

Figure 2.2: Vascular flow profile of flap delay model 38

Figure 2.3: Assessment of angiogenesis in flap delay model $\quad 41$

Figure 2.4: Diameter quantification of collateral vessel $\quad 48$

Figure 2.5: Monocyte recruitment to collateral vessel 49

Figure 2.6: Macrophage recruitment to collateral vessel 50

Figure 2.7: Anti-inflammatory macrophage recruitment to 51

collateral vessel $\quad 51$

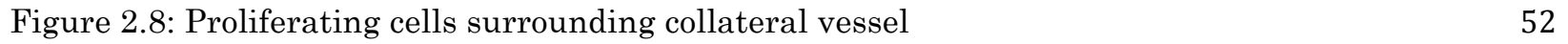

Figure 3.1: Fluorescent labeling of bone marrow derived macrophages $\quad 73$

Figure 3.2: In vivo injection of bone marrow-derived macrophages $\quad 81$

Figure 3.3: Injection of bone marrow-derived macrophages into remodeling inguinal fat pad 85

Figure 3.4: FTY720 application to remodeling inguinal fat pad 86

Figure 3.5: Clodronate depletion of macrophages within inguinal fat pad 88

Figure 3.6: Injection of clodronate liposomes within inguinal fat pad 89

Figure 4.1: Viability of adipocytes in murine tissue 112

Figure 4.2: Viability of adipocytes in human lipoaspirate $\quad 114$

Figure 4.3: Viability of interstitial cells in murine tissue 118

$\begin{array}{ll}\text { Figure 4.4: Viability of interstitial cells in human lipoaspirate } & 120\end{array}$

Figure 5.1: Experimental groups and experimental outline 139

Figure 5.2: Micro-CT scanning of fat grafts 144

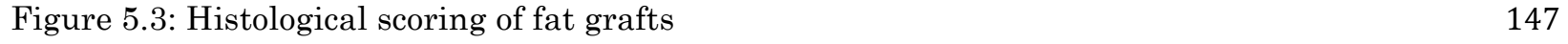

Figure 6.1: Pre-procedure and post-procedure photos of case 163

Figure 6.2: LIVE/DEAD visualization and quantification of control and cryolipolysis-treated tissue

Figure 6.3: Adiposity and vascularity of control and cryolipolysis-treated tissue 166

Figure 6.4: Morphological assessment of control and cryolipolysis-treated tissue 168

Figure 7.1: Fluorescent and phosphorescent properties of BNPs $\quad 184$

Figure 7.2: Application of BNPs to murine full thickness skin wound 190

Figure 7.3: Healing time course and oxygenation levels within wound 191

Figure 7.4: Quantification of wound healing time course 193

Figure 8.1: Two-dimensional versus three-dimensional visualization of adipose tissue 205

Figure 8.2: Photoacoustic microscopy of adipose tissue 215 


\title{
EXAMINATION OF MICROVASCULAR REMODELING TO ENHANCE AUTOLOGOUS FAT GRAFTING
}

\author{
By Scott Alan Seaman
}

"The wise know their weakness too well to assume infallibility: and he who knows most, knows best how little he knows." 
Chapter 1

\section{INTRODUCTION}




\section{Background}

Adipose tissue, or fat, is a complex tissue that serves as both an endocrine signaling organ and a storage organ of excess energy. Adipose tissue is highly vascularized, which provides blood flow to aid in adipose tissue growth/expansion as well as maintaining proper metabolic function. It is well known that excessive adipose tissue has major implications in the pathogenesis of obesity-related disorders such as Type 2 diabetes and the role of the inflammatory system in these disorders is highly studied. Recently, surgeons have started using adipose tissue as a means to reconstruct soft tissue defects due to its innate biocompatibility, its low risk of immune rejection, its high degree of vascularity, and its relative ease of harvest. Central to both of these outlined instances is the marriage of the vascular system and the immune system. The overarching goal of this thesis is to better understand the relationship between microvascular remodeling and the immune cell compartment in adipose tissue to potentially improve autologous fat graft retention. Previous studies have implicated the microvascular system as a crucial determinant in the survival of the grafted tissue, but little to no work has looked at the interplay between immune cell recruitment and microvascular adaptations within adipose tissue. Better knowledge of these interwoven processes may lead to better clinical outcomes of autologous fat grafting. At the end of this introduction section, I will describe how I addressed these biological processes in adipose tissue at large and small scales by using unique tools and techniques in vitro and in vivo. 


\section{Autologous fat grafting}

\section{Clinical motivation}

There exists a significant clinical need for repairing soft tissue defects resulting from pathological conditions, trauma, congenital disorders, and post-operative cancer resections with over 5.7 million reparative surgeries in $2013^{1,2}$. Autologous fat has been regarded as an appealing tissue to use for these surgeries due to its innate biocompatibility, high degree of vascularity, and low risk of immune rejection ${ }^{3}$. Other advantages of using autologous fat include an abundance of donor fat in patients, relative ease of harvest, and a more cost-effective alternative to synthetic or allogeneic implants ${ }^{4,5}$. There has been a dramatic increase of using autologous fat in both large-volume fat grafting utilized in breast and buttock reconstruction, as well as in small-volume fat grafting utilized in facial reconstructive applications ${ }^{6-8}$. In 2011 there were 455,444 autologous fat graft procedures performed worldwide which accounted for $5.5 \%$ of the 8.3 million total nonsurgical procedures ${ }^{9}$. Of the 95,589 breast reconstructive surgeries in 2013 , $20.4 \%$ of these surgeries involved autologous fat for reconstruction ${ }^{2}$.

Despite the popularity of using autologous fat in reconstructive surgeries, fat graft survival and long-term retention is highly variable and unpredictable with studies estimating resorption rates ranging from $25-80 \%{ }^{5}$. To combat the imminent resorption of injected or transferred fat, clinicians often overcorrect the amount of transferred or injected fat up to as much as $140 \%$ so that the final result is in line with the patient's expectations ${ }^{10}$. Still, some patients will require follow-up 
surgeries/fat injections several months after the initial surgery to correct for this resorption. Significant overcorrection to circumvent the resorption process can also increase the incidence of fat necrosis, calcification, and severe infection ${ }^{11-13}$.

\section{Resorption of autologous fat grafts}

This resorption process is poorly understood and the underlying mechanism(s) responsible for long-term volume retention of fat grafts and flaps remains to be elucidated. Advances in survival rate have been limited mainly due to this poor understanding, but proper vascularization and sufficient oxygen/nutrient delivery seem to be the crux of graft and flap survivals. The current paradigm, which is supported by clinical ${ }^{14,15}$ and pre-clinical ${ }^{16,17}$ studies, is that fat survival and longterm volume retention is correlated to the amount of vascularization at the recipient site and the rate at which revascularization within the graft or flap occurs. A prominent theory regarding graft survival is the "diffusion/angiogenesis" theory, which postulates that adipocytes survive via oxygen diffusion for the first 7-14 days post-grafting until angiogenesis occurs to provide a blood supply with oxygen/nutrients to the grafted cells ${ }^{18}$. This theory has led clinicians to inject small, discrete volumes of fat when grafting to prevent fat necrosis that is imminent if the volume of fat is too great. A competing theory conjectures that the dead cellular debris within the graft act as a "matrix" or "scaffold" through which macrophages are recruited and recipient-site stem cells mediate angiogenesis/adipogenesis to retain fat volume ${ }^{18}$. These competing theories may be correct and likely are not 
mutually exclusive processes, but rather working in tandem. Although both of these theories focus on proper vascularization for fat graft survival, little to no work has been conducted examining the changes at the microvascular network level. Studies aiming to improve fat graft retention have tried to exploit these theories without a sound basis of how this revascularization process is occurring.

\section{Scales of fat grafting}

Autologous fat grafting typically manifests itself in two different practices/scenarios: (1) fat grafting, which typically involves non-surgical injection of micro-volumes of fat, and (2) flap transfer, which involved surgical composite tissue transfer of macro-volumes of fat, muscle, and often skin. While both scenarios have differing sizes as depicted in Figure 1.1, there are also fundamental differences between the two. Micro-volumes of fat also called fat grafts consist solely of adipocytes and the adipose tissue interstitial cells. Fat grafts are typically injected to fill small defects ranging from facial creases, small contour deficits, and hemi-facial atrophies. Typically, fat grafts are isolated from one part of the body using a suction lipectomy technique and purified via centrifugation to remove oil and tumescent fluid before injection into the recipient site. While vascular cells are present as part of the interstitial cell compartment, the fat graft does not have an intact vascular supply that is reconnected upon injection. Macro-volumes of fat, termed "flaps", consist of a composite tissue of fat, muscle, and often skin (Figure 1.1) that is transferred to a recipient site. Flaps are considerably larger and are 
used to reconstruct more significant defects such as breasts following a mastectomy. A differentiating factor between flaps and fat grafts, apart from the size, is the presence of an intact vascular supply in a flap that is reconnected upon transfer to the recipient site (Figure 1.1). There are several types of flaps depending on the proportion of tissue (fat vs. muscle), the orientation of the vascular supply system, and the intended use of the flap, which is discussed in detail by Mathes and Nahai ${ }^{19}$. In Chapter 2, we develop a murine model examining microvascular remodeling in a purely adipose tissue flap.
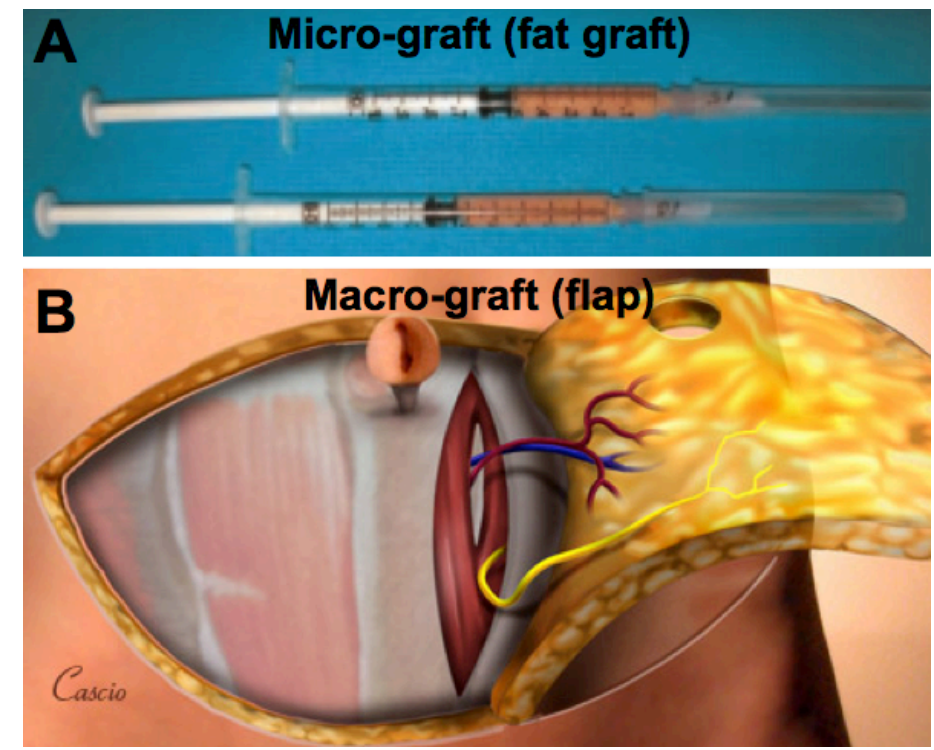

Figure 1.1: Micro-graft (fat graft) vs. macro-graft (flap)

A.) Human fat graft prior to injection with $1 \mathrm{~mL}$ syringe. Graft does not have intact vascular supply and consists solely of adipocytes and interstitial cells. Figure adapted from Cui et al. ${ }^{20}$ B.) Schematic of a macro-graft (flap) abdominal flap composed of skin and adipose tissue with an intact vascular supply. Figure adapted from University Plastic Surgery Copyright 2011.

Techniques to combat autologous fat resorption

Improving vascular retention and mitigating resorption of fat grafts and flaps has been a substantial problem for researchers, clinicians, and the healthcare field for a 
long time. Surgeons and researchers have developed techniques to improve autologous fat retention at both scales (fat grafts and flaps), but many of the techniques are based on gross observation and the mechanism(s) responsible for graft and flap survival are not well understood. While the resulting outcomes of improving the grafts or flaps may be advantageous to overall retentions, it is important to understand the mechanism(s) in order to potentially improve the processes.

Supplementation, or "enhancing", of fat grafts (micro-grafts) with the stromal vascular fraction (SVF), specifically adipose-derived stem cells (ASCs), has garnered significant interest by both researchers ${ }^{3,5,7,8}$ and companies (Cytori Therapeutics, The GID group, among others) in the past decade as a means to improve volume retention and surgical outcomes. The SVF is a compartment of adipose tissue that is rich in preadipocytes, mesenchymal stem cells such as ASCs, endothelial progenitor cells (EPCs), and immune cells including macrophages, T cells, B cells, and mast cells $^{21,22}$. The SVF is generally easy to isolate by using enzymatic digestion with an enzyme such as collagenase ${ }^{23,24}$, centrifugation ${ }^{25}$, mechanical isolation via vigorous shaking ${ }^{26}$, and decanting following density gradient separation ${ }^{27}$. ASCs isolated from the SVF are mesenchymal, multipotent cells with the ability to differentiate down chondrogenic, osteogenic, and adipogenic lineages ${ }^{23,28}$. It is believed that these differentiation capacities contribute to the improvement of graft survival upon supplementation or enhancement with ASCs or the SVF. Yoshimura et al. ${ }^{8}$, among 
others, have used SVF supplementation for the past decade to improve aesthetic outcomes of breast reconstructions.

Transfer of tissue flaps from a patient's donor site to the recipient site immediately incites an ischemic environment and failure to quickly revascularize the flap will lead to necrosis and failure ${ }^{29}$. To combat this possibility of necrosis, surgeons have developed a surgical pre-treatment technique termed "flap delay" that dates back to $16^{\text {th }}$ century when the technique was used in a nasal reconstructive surgery ${ }^{30}$. During flap delay, the main feeding vessel into the flap is ligated (sutured closed to impede blood flow) 10-14 days prior to tissue transfer. It is believed that this alteration in blood flow induces microvascular remodeling within the flap to better withstand an ischemic environment, and, upon transfer the autologous flap has a higher rate of survival ${ }^{31}$. While flap delay is performed routinely in the clinical setting, the findings of improved graft retention are based on observation with little empirical evidence of the vascular mechanism(s) induced by performing the procedure.

\section{Previous work to understand resorption process}

A significant amount of research in the past decade has focused on improving autologous fat graft survival and long-term volume retention on both the micrograft scale ("fat grafts") and the macro-graft scale ("flaps"). Previous studies on delayed macro-graft flaps and their survival have focused on changes at the cellular and protein level ${ }^{31}$ - citing increases in angiogenic/growth factor secretion (VEGF, 


\section{CHAPTER 1: INTRODUCTION}

bFGF) $)^{32,33}$, upregulation of angiogenic transcription factors (HIF-1- $\left.\alpha\right)^{34}$, and increases in endothelial progenitor cell recruitment ${ }^{35}$. Studies examining micrografts ("fat grafts") and their survival following grafting have focused on gross visualization and volume retention of the fat, blood vessel ingrowth into the grafted fat, and angiogenic protein secretion assays ${ }^{36}$. Other studies suggest that supplementing fat micro-grafts with stromal vascular fraction (SVF) isolated using collagenase improves graft survival ${ }^{5,7,37}$, but it is unknown how collagenase digestion affects adipocytes and the interstitial cells that comprise the SVF. Little to no studies have examined changes at the vascular network level, and specifically in regards to structural remodeling of the microvessels and microvascular networks.

Here, we aim to study angiogenesis and arteriogenesis at both the micro-graft and macro-graft scale so that we may harness or manipulate this innate remodeling response to improve autologous fat graft survival.

\section{Microvascular system}

\section{Overview of microvascular system}

The microvasculature is a ubiquitous organ system within the body that is responsible for distributing oxygen and nutrients to tissues as well as removing waste products from perfused $\operatorname{areas}^{38}$. The microvascular system consists of arterioles, capillaries, and venules, and forms a branching, tapered network in order to distribute blood efficiently to organs and tissues ${ }^{39,40}$. While organs have differing microvascular architectures, the fundamental elements (arterioles, 
capillaries, and venules) of the microcirculation remain consistent across all vascular beds ${ }^{39,41}$.

Arteries are responsible for bringing oxygenated blood away from the heart and it is these arteries that give rise to smaller arterioles within the microcirculatory system. Arterioles are generally considered to be less than $500 \mu \mathrm{m}$ in diameter in humans and are coated with smooth muscle cells that are circumferentially arranged around the internal endothelial layer that blood passes through $^{39}$. Arterioles have the ability to modulate their vascular tone and actively manipulate their diameters, an important attribute used to regulate proper blood flow to organs and tissues ${ }^{42,43}$. The pre-capillary (terminal) arterioles that drain into the capillary bed are generally considered to be less than $30 \mu \mathrm{m}$ in diameter for humans ${ }^{39,43}$.

Capillaries are the smallest blood vessels in the human body and consist of a single layer of endothelial cells (ECs) forming a tube with diameters ranging from 4$10 \mu \mathrm{m}$ in humans ${ }^{44}$. The capillary bed, an extensive network of capillaries, is where exchange of gases, nutrients, and water occur between the bloodstream and the tissues $^{39,40}$. The capillary bed is well-suited for this exchange because of the highly permeable endothelial barrier ${ }^{40}$. Another important cell type of the microvasculature is the pericyte which is an abluminally-located, stabilizing perivascular cell located on capillaries and post-capillary venules ${ }^{45-48}$. Pericytes possess long, extending processes and previous reports have highlighted their ability to regulate capillary blood flow patterns, assist in the guidance of 
extravasated leukocytes, and affect ECs and neighboring cells via paracrine effects $^{45,46,49-52}$.

Blood drains into post-capillary venules (lack a smooth muscle cell layer), which allow extravasation of recruited circulating blood cells (i.e. leukocytes) into surrounding tissue ${ }^{39,53}$. Venules are also permeable to plasma proteins and serve as a major site for transvascular protein exchange ${ }^{39,54}$. As the post-capillary venules increase in caliber, they gain a coat of irregularly arranged smooth muscle cells ${ }^{55}$. Venules are generally two to three times larger in diameter than the corresponding arteriole and have been reported to be more prevalent than arterioles in specific tissues $^{39}$.

\section{Microvascular remodeling}

The highly orchestrated microvascular system is essential to maintain tissue health by delivering nutrient-rich blood (arterioles), permitting exchange of nutrients and gases (capillaries), and allowing extravasation of blood-derived cells and plasma proteins (venules) ${ }^{56,57}$. The microvasculature is a dynamic system that is able to adapt its structure and function in response to biochemical/biomechanical cues, often to meet the metabolic demands of the perfused tissue. Compensatory changes of the vascular network can be beneficial, as is the case with increased capillary density in skeletal muscle following increased exercise ${ }^{58}$, or detrimental and pathological, such as during rampant, aberrant vascular growth during proliferative diabetic retinopathy ${ }^{59,60}$ or tumor progression ${ }^{61}$. Because the microcirculation is the 
interface of the bloodstream and the perfused tissue, adaptations within the microvasculature can often dictate whether proper function of the tissue will be restored or if a pathological case will be exacerbated.

Microvascular remodeling can be divided into five events or processes: angiogenesis, arteriogenesis, vasculogenesis, intussusception, and regression. Each of these processes are elicited by different environmental stimuli or cues. For example, prolonged ischemia and subsequent hypoxia within a tissue or organ can initiate new blood vessel growth from pre-existing blood vessels (angiogenesis) ${ }^{62-65}$, while an increase in fluid shear stress (FSS) in small arterioles can induce the enlargement of this arteriole (arteriogenesis) ${ }^{66-68}$. Angiogenesis is induced in response to endothelial cells sensing hypoxia, while arteriogenesis is induced in response to physical/mechanical forces sensed by the endothelial cells within the arteriole ${ }^{69}$. Both processes will be discussed in further detail below. Vasculogenesis, intussusception, and regression are also important processes of the microvascular remodeling continuum and respond to different environmental cues ${ }^{57}$. Vasculogenesis, the de novo formation of blood vessels, manifests during embryonic development when angioblasts differentiate into endothelial cells to form primitive vascular networks ${ }^{70}$. Intussusception is the division of pre-existing vessels into smaller caliber vessels and is important in the development of many organs, wound healing, and tumor angiogenesis ${ }^{71-73}$. Finally, vessel regression is the pruning of pre-existing blood vessels to aid in the creation and maintenance of a functional, organized vascular network ${ }^{57,74}$. Together, these microvascular remodeling 
processes exemplify the dynamic nature of the microvascular system and highlight their importance in creating functional, patent vascular networks. While other modes of microvascular remodeling are indeed important, this thesis will focus on angiogenesis and arteriogenesis

\section{Angiogenesis}

Angiogenesis, the growth of new blood vessels from pre-existing blood vessels, is required for development, growth, and repair of all tissues within the body ${ }^{62,75}$. It is well known that hypoxia (oxygen deficiency) is a powerful stimulus for vascular growth $^{62-65}$. During the embryonic and developmental stage of life, the vascular bed relies on sprouting and maturation of endothelial cells to form a patent vasculature in order to supply nutrients for tissue development and growth ${ }^{62,76,77}$. During the growth and expansion of the tissue, simple diffusion of oxygen is no longer sufficient or able to the necessary oxygen to the tissue ${ }^{62}$. To circumvent this limitation, the presence of hypoxia induces the activation of a signaling network through hypoxia inducible transcription factors (HIFs) with one of the most studied HIFs being hypoxia-inducible factor 1 (HIF-1 $)^{62,78}$. Upon activation, HIFs activate numerous angiogenic genes but the most prominent, and arguably most important in the context of angiogenesis, is vascular endothelial growth factor A (VEGF-A) ${ }^{62,79}$. VEGF-A is a potent angiogenic protein that signals through the Notch1-DLL4 signaling axis to activate endothelial cell sprouting and eventually form patent, mature vascular networks ${ }^{80-82}$. 
Some of the work presented in this thesis focuses on angiogenesis at the network level, that is, observations of changes of the capillary beds themselves. Significant research has been conducted examining angiogenesis at the transcription factor and protein level but this will not be a focus of this work. Previously, our group has studied angiogenesis and network level changes in the mouse retina ${ }^{83}$ and the mouse spinotrapezius muscle ${ }^{84-86}$. Here, we focus on angiogenesis and the adaptation of the capillary network within adipose tissue and aim to understand microvascular remodeling to improve autologous fat grafting.

\section{Arteriogenesis}

Arteriogenesis, the expansion in diameter of pre-existing arteries/arterioles, is a compensatory mechanism to increase blood flow to a tissue experiencing a loss/reduction of blood flow and nutrients ${ }^{69,87}$. The reduction of blood flow to the tissue is usually caused by blood vessel occlusion such as the case in peripheral artery disease $(\mathrm{PAD})$ in skeletal muscle ${ }^{88}$. After occlusion, blood is rerouted to neighboring collateral vessels and it is these vessels that initiate the process of arteriogenesis. The endothelial cells lining these collateral vessels experience an increase in fluid shear stress (FSS) and blood pressure, which, in turn, activates the endothelial cells that comprise the lumen of the blood vessel ${ }^{69,87}$. Interestingly, arteriogenesis appears to be independent of the presence of hypoxia and is solely activated by the increase in FSS $^{69}$. This activation of the endothelial cells induces upregulation of genes encoding for the production of chemoattractants, cytokines, and adhesion molecules, with the goal of recruiting circulating cells from the blood 


\section{CHAPTER 1: INTRODUCTION}

stream $^{69,89}$. A major chemoattractant protein, monocyte chemoattractant protein-1 (MCP-1), is immobilized at the endothelial cell surface, which results in significant recruitment of monocytes ${ }^{90,91}$. Endothelial cell activation in response to an increase in FSS also induces expression of selectins, intercellular adhesion molecule-1 (ICAM-1) and ICAM-2, and vascular cell adhesion molecule-1 (VCAM-1) ${ }^{69,92}$. Coupling of the MCP-1 gradient with these selectins and adhesion molecules fosters an environment for monocyte recruitment and adhesion to luminal surface of the blood vessel. Following adhesion of the recruited monocytes through receptor-ligand binding (i.e. Mac-1 with ICAM-1) ${ }^{90}$, the monocyte will extravasate (transmigrate) through the vessel wall into the interstitial space of the tissue ${ }^{93}$. Monocytes secrete potent proteases such as matrix-metalloproteinase-2 (MMP-2) and MMP-9 that degrade nearby extracellular matrix and allow for expansion of the collateral vessel wall ${ }^{94}$. Once extravasating from the vessel, the monocytes can differentiate into macrophages, which can secrete growth factors (i.e. fibroblast growth factor-2 (FGF2)) to stimulate SMC and EC proliferation and expansion of the collateral vessel itself $93,95,96$. Macrophages also provide cues for SMCs to differentiate from a mature, contractile phenotype to a more synthetic, proliferative phenotype ${ }^{92}$. This concerted bone marrow cell-directed inflammatory process also includes $\mathrm{T}$ cells ${ }^{97,98}$, natural killer (NK) cells ${ }^{98}$, neutrophils ${ }^{94,99}$, and possibly mast cells ${ }^{100}$, but the specific focus in this thesis will be on the involvement of macrophages during this process. For a more thorough review of the arteriogenesis process please see the articles by Carmeliet ${ }^{101}$ and by Schaper and Scholz ${ }^{95}$. 
The work presented in Chapters 2 and 3 focuses on arteriogenesis and the interactions with the recruited and tissue-resident macrophage populations within adipose tissue, which has not been studied extensively in the past. Specifically, we characterize how a pre-existing collateral arteriole structurally adapts in concert with the recruited monocytes and macrophages. Previous work in our lab has studied arteriogenesis in skeletal muscle using an ischemic ligation murine model ${ }^{84-86,102}$, but our work in this thesis aims to understand arteriogenesis in a clinically relevant murine model of autologous fat grafting.

\section{Adipose tissue anatomy and microstructure}

Adipose tissue is a metabolically active, complex organ that is essential to maintain and regulate metabolism in both health and disease ${ }^{103,104}$. As an endocrine organ, adipose tissue secretes various adipokines (i.e. leptin, resistin, adiponectin) to aid in this regulation of total body energy and lipid homeostasis ${ }^{105-107}$. Two types of adipose tissue exist in humans and mice, brown adipose tissue (BAT) and white adipose tissue (WAT) ${ }^{108,109}$. BAT is thermogenic meaning that BAT can create heat from the lipids that it stores, while the main objective of WAT is to store excess triglycerides to be used as energy ${ }^{108,110}$. BAT is prevalent in small mammals that cannot produce heat from shivering and in newborns ${ }^{111}$. WAT is far more prevalent in human adults and is located in two primary depots or locations, subcutaneous adipose tissue depots and visceral adipose tissue depots ${ }^{112}$. Subcutaneous adipose tissue is found below the skin, while visceral adipose tissue is found lining internal organs and has been implicated in many obesity-related diseases ${ }^{105}$. WAT, 
specifically subcutaneous adipose tissue of human and mice, will be the main subject of this dissertation.

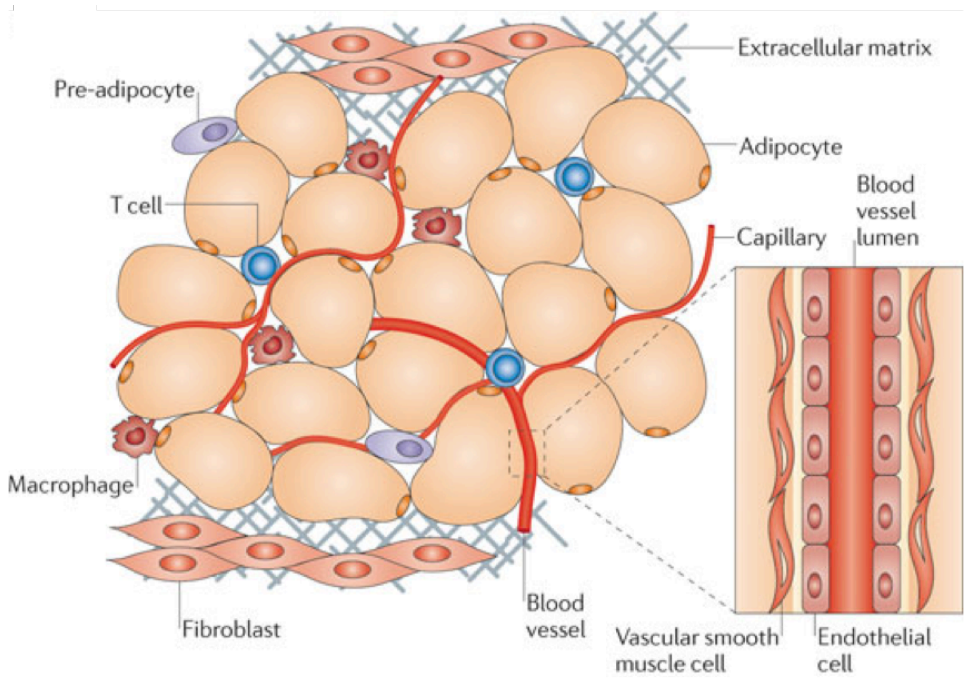

Figure 1.2: Cellular components of adipose tissue

The microenvironment of adipose tissue is heterogeneous with numerous cell types including adipocytes, fibroblasts, vascular cells, pre-adipocytes, and immune cells. Figure adapted from Ouchi et al. ${ }^{106}$.

It is well known that the microenvironment of adipose tissue is heterogeneous and contains numerous cell types that are highly coordinated to comprise the overall functional unit ${ }^{106,113}$. Figure 1.2 depicts the adipose tissue microenvironment and illustrates the main components of adipose tissue ${ }^{106}$. The most prominent cell of adipose tissue is the adipocyte, which is an oblong-shaped cell whose primary role is the storage of energy in the form of lipids ${ }^{113}$. Notably, adipose tissue is highly vascularized with a well-developed capillary bed (endothelial cells) and a significant arteriole and venule presence (vascular smooth muscle cells and endothelial cells) ${ }^{106,114}$. It is thought this high degree of vascularity permits the rapid growth and expansion of adipose tissue during times of nutrient excess $^{114}$. Also present in adipose tissue are pre-adipocytes (adipocyte progenitor 
cells) that are able to differentiate into adipocytes during adipogenesis and provide a source for adipocytes during tissue expansion ${ }^{115}$. Tissue-resident immune cells (macrophages, T cells, mast cells, dendritic cells, among others) are present within adipose tissue and have important roles in maintaining local tissue homeostasis ${ }^{16,117}$. In obese adipose tissue the immune cell compartment is imbalanced and has been implicated in obesity-related local and systemic inflammation. Fibroblasts also play an important role within adipose tissue by producing extracellular matrix that can aid or hinder adipose tissue expansion ${ }^{106}$.

The focus of this thesis will be on the adipocytes, vascular cells, monocytes, and macrophages of the adipose tissue.

\section{Monocytes and macrophages}

Monocytes and macrophages are phagocytic cells that play vital and distinct roles within the body's immune system ${ }^{118}$. The mononuclear phagocytic system, introduced in the 1960s by van Furth et al., suggests that tissue-resident macrophages are derived from monocytes and these monocytes are derived from hematopoietic stem cells (HSCs) residing in the bone marrow ${ }^{119,120}$. Monocytes, the intermediate cell in the mononuclear phagocytic system, are phagocytic leukocytes that play various roles in the innate immune system ranging from antigen presentation to $\mathrm{T}$ cells, differentiation into macrophages after extravasation from the vasculature, and clearing cellular debris during an inflammatory event ${ }^{121-123}$. There are several phenotypes of macrophages with roles ranging from the ingestion of microbes during the inflammatory process ${ }^{118,124}$, secretion of growth factors and 


\section{CHAPTER 1: INTRODUCTION}

cytokines to aid in growth and proliferation ${ }^{121,125-127}$, and activation of other immune cells including $\mathrm{T}$ cells ${ }^{128}$. Recent studies have suggested that macrophages have different origins than originally proposed by van Furth et al. ${ }^{119}$ and have shown the ability of tissue-resident macrophages to self-renew ${ }^{129}$. While the origin of macrophages is interesting (and currently still evolving), this work focuses on the role that macrophages play during microvascular remodeling and thus the origin of macrophages will not be discussed further. For a more extensive discussion on the origin(s) of macrophages, please see Hashimoto et al. ${ }^{129}$.

\section{Macrophage phenotypes}

The classification of macrophages is a constantly evolving and debated topic within the immunology field and can be loosely defined into a continuum of phenotypes ${ }^{130-}$ 133. The current paradigm is to classify macrophages as either the M1 or M2 phenotype, which was motivated by the T-helper cell nomenclature ${ }^{131,132,134}$. M1 macrophages, also designated "classically activated macrophages", are considered the pro-inflammatory macrophage ${ }^{131,134,135}$. M1 macrophages are stimulated by interferon gamma (IFN-Y), microbial products, lipopolysaccharide (LPS), and tumor necrosis factor-alpha (TNF-a) (among others) and secrete pro-inflammatory cytokines such as interleukin-1 (IL-1), IL-6, IL-12, IL-23 to trigger type I inflammation and Th1 response ${ }^{131,132,135}$. M2 macrophages, often referred to as "alternatively activated macrophages", are considered to be anti-inflammatory macrophages and aid in the inflammatory resolution process ${ }^{133,136}$. M2 macrophages 


\section{CHAPTER 1: INTRODUCTION}

are stimulated by IL-4, IL-10, IL-13, glucocorticoid (among others) and secrete antiinflammatory cytokines such as transforming growth factor-B (TGF-B) and IL-10 to aid in type II inflammation and the Th2 response ${ }^{133,135,136}$. Recent studies have expanded the M2 classification into four separate phenotypes, M2a, M2b, M2c, M2d, in which the classically defined M2 phenotype is now the M2a phenotype ${ }^{136}$. Other work by Kadl et al. has proposed a separate, distinct macrophage phenotype, $\operatorname{Mox}^{135}$.

In Chapters 2 and 3, we examine the role of macrophages, specifically M2 macrophages, in adipose tissue and their presumed role in microvascular remodeling. To characterize the macrophages within the adipose tissue, we rely on immunofluorescence by using antibodies targeted against cell surface markers such as cluster of differentiation (CD)68, CD206, and F4/80. For a thorough examination of techniques to classify and characterize macrophage phenotypes, please the reviews by Murray et al. ${ }^{132}$ and Corliss et al ${ }^{121}$.

\section{Macrophage and microvascular remodeling}

While it is well known that macrophages are an important part of the inflammatory process, recent studies have suggested a significant role of macrophages in microvascular growth and remodeling. Recent studies suggest a paracrine effect of macrophages angiogenesis as evidenced by the secretion of numerous proangiogenic growth factors and cytokines such as basic fibroblast growth factor (bFGF), VEGFA, angiopoietin-2 (Ang-2), and insulin-like growth factor-1 (IGF-1), among many 
others $^{121}$. Interestingly, the polarization state of the macrophages can influence the profile of cytokines secreted, which may imply that different macrophage subsets influence different stages of angiogenesis ${ }^{137}$. Recent work may connect macrophages to the process of arteriogenesis via paracrine signaling. Ischemia-inducing ligation studies conducted in murine skeletal muscle have shown recruitment of monocytes to the injured tissue followed by differentiation of these monocytes into macrophages, specifically M2 macrophages. Studies by Awojoodu et al. and Bruce et al. suggest that these recruited monocytes and macrophages have a paracrine effect on the remodeling arterioles during arteriogenesis ${ }^{86,138}$. Takeda et al. show that expansion of M2 macrophages within skeletal muscle following hindlimb ischemia aid in collateral vessel formation via release of arteriogenic factors to enhance SMC recruitment and growth ${ }^{139}$. Macrophages have also been shown to directly interact with vascular cells such as pericytes, vascular SMC, and endothelial cells, during microvascular growth and remodeling. For example, macrophages can form facilitating "bridges" between endothelial cells during sprouting angiogenesis in mouse and zebrafish hindbrain ${ }^{140}$. In an inflammation model of the mouse ear, macrophages were "instructed" by resident arteriolar and capillary pericytes after extravasating from the post-capillary venule ${ }^{49}$.

Based on these previous studies and others not mentioned, it is apparent that macrophages have a significant role in microvascular growth and remodeling and is thus a central thread in this thesis work. 


\section{Overview of thesis}

Little to no studies have examined changes at the vascular network level, and specifically in regards to structural remodeling of the microvessels and microvascular networks. Here, we aim to study angiogenesis and arteriogenesis at both the micro-graft and macro-graft scale so that we may harness or manipulate this innate remodeling response to improve autologous fat graft survival. Our overarching strategy of assessing both angiogenesis and arteriogenesis is unique, as previous studies have only focused on one or the other without considering the differential factors and outcomes associated with these two different modes of microvascular remodeling. Our evaluation of vascular growth and remodeling, while considering the contributions of the innate immune system (monocytes and macrophages) in these processes, is also highly innovative and is not typically considered in the setting of fat grafting.

Herein, I present my work on microvascular remodeling and the contribution of the immune system on autologous fat survival. Throughout the course of this work, I used a number of innovative and unique techniques to understand the vascular remodeling response within adipose tissue. We formulated a clinically relevant murine model of flap delay to examine vascular remodeling events in a macro-graft using several tools and methods. Our lab has extensive experience imaging whole-mount immunofluorescently-labeled tissue (skeletal muscle) using confocal microscopy ${ }^{85,86,102}$. This technique afforded us the ability to image the vascular networks in their innate conformation, to visualize cell-type specific recruitment to the tissue, and allowed us to visualize vascular changes post-surgery 
or intervention. Previous studies have not looked into this level of detail of vascular remodeling or cell recruitment to the adipose tissue. We successfully adapted this whole-mounting technique for use in fat, which has provided us the ability to visualize microvascular responses and immune cell recruitment in concert. We coupled the whole-mounting technique with cell-type specific transgenic fluorescent reporter mice, which allowed us to visually track immune cell (CX3CR1 $1^{+/ G F P}$ reporter mice) recruitment to remodeling arterioles and venules (NG2-DsRed reporter mice) with spatial resolution.

Building on our published flap delay model and our preliminary findings that M2 macrophages are partly responsible for the observed microvascular remodeling, we performed several interventions as gain-of-function and loss-of-function studies. As gain-of-function studies, we injected in vitro differentiated M2 macrophages ${ }^{135}$ into the remodeling fat and we delivered a novel FDA approved drug, FTY720, to the remodeling fat. Both studies were novel interventions that helped us confirm our original findings in the murine flap delay model. For loss-of-function studies, we applied clodronate liposomes to the remodeling fat to diminish the number of macrophages in the fat pad, something that has not been conducted in adipose tissue before.

To understand the effect of collagenase digestion and SVF supplementation on micro-grafts, we used x-ray microtomography (micro-CT) to serially and noninvasively track the volume retention of human adipose tissue in a modified, published xenograft model ${ }^{141,142}$. This afforded the ability to serially track the 
volume of the grafted fat without the need to sacrifice the animal at each time point, something that has not been performed extensively in the literature.

In Chapter 2, we adapted a previously published model to formulate a new pre-clinical model of flap delay to study microvascular remodeling within the flap using aforementioned techniques and tools. In our model, we selectively ligate the main artery, the epigastric artery, which supplies the subcutaneous inguinal fat pad with blood. This reduction in blood flow is similar, though not exact, to a clinical case in which a surgeon performs "flap delay" and alters blood flow in a flap to be transferred. By altering blood flow into the murine inguinal fat pad we were able to observe the microvascular remodeling response downstream of this ligation and owe the responses observed to arteriogenesis rather than angiogenesis. We observed an enlargement in diameter in the collateral vessels and did not observe an increase in angiogenesis in the fat pad. In agreement with our studies in skeletal muscle we observed a preferential recruitment of anti-inflammatory macrophages to the remodeling tissue. Chapter 2 formed the basis for a journal article that has been published in Microcirculation.

In Chapter 3 we build on our original findings in Chapter 2 that antiinflammatory macrophages are primarily responsible for the remodeling responses observed in our model. We performed a series of gain-of-function and loss-offunction studies in which we increase or decrease the number of macrophages in the remodeling tissue. First, we directly injected in vitro differentiated M2 macrophages into the inguinal fat pad and observed an even more pronounced arteriogenic 
response (increase in collateral vessel enlargement). In a separate set of experiments, we apply a novel drug, FTY720, to the inguinal fat pad by implanting drug-loaded PLAGA films. In skeletal muscle FTY720 has been shown to preferentially recruit anti-inflammatory monocytes to remodeling tissue. We observed an increase in the number of M2 macrophages to the tissue and again observed an increase in arteriogenic response, which is consistent with our direct injection studies above. We also performed a "macrophage knockdown" experiment in which we inject clodronate liposomes to the inguinal fat pad to reduce the number of macrophages present in the tissue. We observed a $60-70 \%$ reduction in the number of macrophages present in the tissue and a diminished arteriogenic response after ligation, likely due to the decrease in macrophage presence. Taken together, these data and experiments indicate the importance of macrophages, specifically M2 macrophages, in the remodeling response induced following flap delay. Chapter 3 will form the basis for a paper that will be submitted to Microcirculation as a follow-up study to our original publication.

In Chapter 4, we examine human and murine micro-grafts and the effect of collagenase digestion and time ex vivo on the viability of the adipocytes and interstitial cells. Despite the prevalence of using collagenase digestion as a means to isolate the SVF, a standardized time for collagenase digestion has not been reached with times varying between 20 and 90 minutes $^{143-145}$ and the effect of digestion time on the adipocytes and interstitial cells has not been examined thoroughly. Our findings in vitro indicate that increasing durations of collagenase 
time are detrimental to adipocytes and interstitial cell health - increasing digestions time leads to a decrease in adipocyte and interstitial cell viability. This result has implications in the plastic surgery and laboratory settings, as collagenase digestion is used routinely for SVF isolation in the clinic and adipocyte studies in the laboratory. This study highlights the importance of monitoring the enzymatic activity of the digestion buffer prior to performing experiments in a laboratory setting on interstitial cells/adipocytes, or using the cells as part of a fat grafting procedure in a clinic. The findings reported in Chapter 4 have been previously published in Plastic and Reconstructive Surgery journal.

In Chapter 5 we aimed to build on our findings from Chapter 4 by determining the effect of collagenase digestion on in vivo graft survival. In Chapter 4 we determined that prolonged collagenase digestion reduces adipocyte and interstitial cell viability. We sought to determine if pre-digestion of the adipocytes that comprise the grafts reduces the overall volume retention of the graft postimplantation. Further, we aimed to determine if SVF supplementation aids in the volume retention of these implanted grafts. We utilized a published murine xenografts model in which we implanted human adipose tissue (collagenase digested or not, SVF supplemented or not) on top of murine scalps. We performed micro-CT scans at various time points up to twelve weeks and calculated estimated volume of the adipose tissue by three dimensional volume rendering. We harvested the tissue at the terminal time point and recorded the mass of the tissue and histologically evaluated the grafts. We found that collagenase pre-digestion of the 
adipocytes significantly reduces the volume of fat after implantation with nearly no adipose tissue left in the collagenase digested groups after two weeks. SVF supplementation improved the retention of the collagenase-digested group but was not able to rescue the volume retention to a level that was observed in non-digested tissue. The results of $H \& E$ staining and masses of the grafts were consistent with observation from the micro-CT scans. Chapter 5 will form the basis for an article to be submitted to Plastic and Reconstructive Surgery.

In Chapter 6, we were presented with a unique case study in which we applied techniques learned in Chapter 4 to understand a pathological condition which presents as a side effect following a commonly used adipose tissue reduction treatment. CoolSculpting®, a non-invasive technique that uses cold exposure to reduce adiposity within a patient has a rare side effect termed paradoxical adipose hyperplasia $(\mathrm{PAH})$ in which the adipose tissue expands following treatment. We obtained tissue samples from a patient that experienced PAH and were able to compare cryolipolysis-treated adipose tissue to control (untreated) tissue. We used techniques and immunofluorescent stains optimized in Chapter 4 to assess adipocyte and interstitial cell viability across the two treatment groups. Our findings from this case study were published in the Aesthetic Surgery Journal and are the basis of Chapter 6 .

Chapter 7 describes a collaborative project with Dr. Cassandra Fraser's chemistry lab at the University of Virginia. Her lab has formulated a biomaterial that is oxygen-sensitive and is responsive to physiological oxygenation levels. In 
collaboration with members of her lab, we designed an imaging platform coupled with oxygen-sensitive nanoparticles for use as a wound diagnostic device. The overall goal of the project is to design a device capable of resolving local oxygenation levels within a wound bed to aid in wound diagnosis in the clinic. We believe that the device has applications in the plastic surgery field as well ranging from diagnosis of proper oxygenation levels within a transferred tissue flap or the monitoring of an evolving burn or injury. We obtained promising results in these preliminary proof-of-concept studies with the ability to resolve oxygenation levels within a murine full thickness skin wound. These findings, along with the material characterization studies, are being submitted to the Journal of the American Chemical Society and comprise Chapter 7 of the thesis.

Lastly, in Chapter 8, I discuss my overarching goal of these studies improving autologous fat graft retention. First, I discuss the key contributions to the field as well as extended applications that arose from this body of work. I continue the chapter with a discussion of exciting next steps for this line of work and conclude the chapter with final reflections about these studies. 


\title{
Chapter 2
}

\section{MACROPHAGE RECRUITMENT AND POLARIZATION DURING COLLATERAL VESSEL REMODELING IN MURINE ADIPOSE TISSUE}

\author{
Acknowledgements: Yiqi Caoa, Chris A. Campbellb, Shayn M. Peircea,b \\ ${ }^{a}$ Department of Biomedical Engineering, University of Virginia \\ ${ }^{b}$ Department of Plastic Surgery, University of Virginia
}

The text included in this chapter has been published here:

Seaman SA, Cao Y, Campbell CA, Peirce SM. Macrophage recruitment and polarization during collateral vessel remodeling in murine adipose tissue. Microcirculation 2016; 23(1): 75-87. 


\begin{abstract}
Objective

During autologous flap transplantation for reconstructive surgeries, plastic surgeons use a surgical pre-treatment strategy called "flap delay", which entails ligating a feeding artery into an adipose tissue flap 10-14 days prior to transfer. It is believed that this blood flow alteration leads to vascular remodeling in the flap, resulting in better flap survival following transfer; however, the structural changes in the microvascular network are poorly understood. Here, we evaluate microvascular adaptations within adipose tissue in a murine model of flap delay.

\section{Methods and Results}

We used a murine flap delay model in which we ligated an artery supplying the inguinal fat pad. Although the extent of angiogenesis appeared minimal, significant diameter expansion of pre-existing collateral arterioles was observed. There was a 5 -fold increase in recruitment of $\mathrm{CX} 3 \mathrm{CR} 1^{+}$monocytes to ligated tissue, a 3 -fold increase in $\mathrm{CD}^{+} / \mathrm{CD} 206^{+}$macrophages in ligated tissue, a $40 \%$ increase in collateral vessel diameters supplying ligated tissue, and a 6 -fold increase in the number of proliferating cells in ligated tissue.

\title{
Conclusions
}

Our study describes microvascular adaptations in adipose in response to altered blood flow and underscores the importance of macrophages. Our data supports the development of therapies that target macrophages in order to enhance vascular remodeling in flaps. 


\section{Introduction}

The microvasculature is a dynamic system that changes its function and structure in response to biochemical and biomechanical stimuli. Prolonged ischemia, (e.g. the loss of blood flow to a region of tissue), is a particularly potent stimulus for angiogenesis, the formation of new blood vessels from pre-existing blood vessels ${ }^{146-}$ 148 and arteriogenesis, the process by which pre-existing arteries/arterioles remodel to increase their diameter $68,95,149$. Angiogenesis and arteriogenesis are capable of restoring blood flow to ischemic tissue ${ }^{95,150}$, providing injured tissue with the necessary nutrients to regenerate, and protecting against future ischemic damage ${ }^{151}$. While angiogenesis and arteriogenesis are physiological processes that occur in health (e.g. during exercise $\mathrm{e}^{91,152}$ and menstruation ${ }^{153}$ ) and during disease (e.g. in response to ischemic insult $68,95,149$ and endometriosis ${ }^{154}$ ), the plastic surgery community has developed surgical strategies that presumably leverage these processes to aid in the repair of soft tissue defects through the use of autologous flaps.

Soft tissue defects commonly result from trauma, congenital disorders, or post-operative cancer resections ${ }^{1}$. Autologous flaps, which can be composed of fat, muscle, and/or skin, are surgically transferred from one region of the body to the site of the defect for reconstruction. Partial autologous flap loss due to inadequate blood flow can lead to multiple operations and a persisting defect. A pretreatment surgical technique termed "flap delay" involves the ligation of the main feeding vessel into the fat 10-14 days prior to tissue transfer to the recipient site. It is 
believed that this alteration in blood flow leads to growth and remodeling of the microcirculation, which allows for transfer of more tissue and improves autologous flap survival ${ }^{155,156}$. Although flap delay is performed routinely in the clinical setting and has been for many years, dating back to the first delayed flap for nasal reconstruction by Gaspare Tagliacozzi in the $16^{\text {th }}$ century ${ }^{30}$, the type of vascular remodeling that occurs within an autologous flap (angiogenesis, arteriogenesis, or both) and the types of vessels that undergo remodeling within the network (i.e. arterioles, capillaries, and/or venules) remain in question. Although the autologous flap is a composite tissue, the majority of the volume comes from the adipose tissue itself, and therefore a purely "adipose flap" will be the subject of our studies.

Prior studies have focused on the cellular composition and protein levels within an autologous flap, examining metabolic changes of the adipocytes, angiogenic protein secretion (VEGF, bFGF) ${ }^{33}$, upregulation of hypoxia-inducible factor $1-\alpha^{34}$, and increased recruitment of endothelial progenitor cells ${ }^{35}$. Other studies have examined vessel dilation responses following vascular delay by using non-invasive laser Doppler flowmetry ${ }^{157}$ and perfused tissue sections ${ }^{158}$; however, few studies to date have examined how the microvascular network dynamically and structurally adapts in concert with the immune cell compartment following ischemic ligation in a clinically relevant model of flap delay.

In this study, we investigated how the microvasculature in a purely adipose flap remodels - at the network level - in response to ischemic ligation of a feeding artery. By evaluating both angiogenesis and arteriogenesis, as well as immune cell 
recruitment in a published murine model of flap delay ${ }^{147}$, our data show that arteriogenesis predominates over angiogenesis and that macrophages aid in the arteriogenic response following recruitment to the delayed adipose flap after 1-3 days. We examine the remodeling response at early time points to observe the interplay between immune cell recruitment and subsequent differentiation with the microvascular remodeling response. Understanding how the microvasculature of adipose tissue structurally adapts in response to ligation can suggest therapeutic mechanism(s) to further enhance autologous flap survival following flap delay, and improve long-term volume retention which will lead to better patient outcomes, fewer corrective procedures, and reduced cost burden on the healthcare system. 


\section{Materials and Methods}

\section{Inguinal fat pad ligation surgery}

All procedures were performed in accordance with the Institutional Animal Care and Use Committee of the University of Virginia. Eight to sixteen week old C57BL/6 mice were used for all studies, unless otherwise specified, with the number of mice denoted in figure legends. A modified, previously published inguinal fat pad ligation model ${ }^{147}$ was used. Briefly, mice were anesthetized with ketamine/xylazine/atropine $(60 / 4 / 0.2 \mathrm{mg} / \mathrm{kg})$ and a $1.5 \mathrm{~cm}$ incision was made in the skin overlying the left inguinal fat pad using a scalpel blade. Skin and underlying connective tissue were carefully undermined using blunt dissection to expose the epigastric artery, which feeds the inguinal fat pad. The murine inguinal fat pad is a closed-network with one main feeding artery and typically three to four smaller collateral vessels that enter the lateral side of the fat pad from the skin (Figure 2.1A). A 10-0 nylon suture (Ethicon, Somerville, NJ) was used to ligate the epigastric artery after carefully separating the artery from its paired vein. The epigastric artery was severed following ligation, and loss of blood flow was confirmed visually using a surgical microscope under 3x magnification. The incision was closed with 8-0 nylon suture (Ethicon, Somerville, NJ). At subsequent time points (30 minutes, 12 hours, 24 hours, 72 hours), adipose tissue was harvested from euthanized mice, as described below. Sham surgery was performed on the contralateral side of each mouse. For the sham surgery, a 10-0 nylon suture was passed underneath the epigastric artery but was not tied, and the incision was closed with 8-0 nylon suture. 

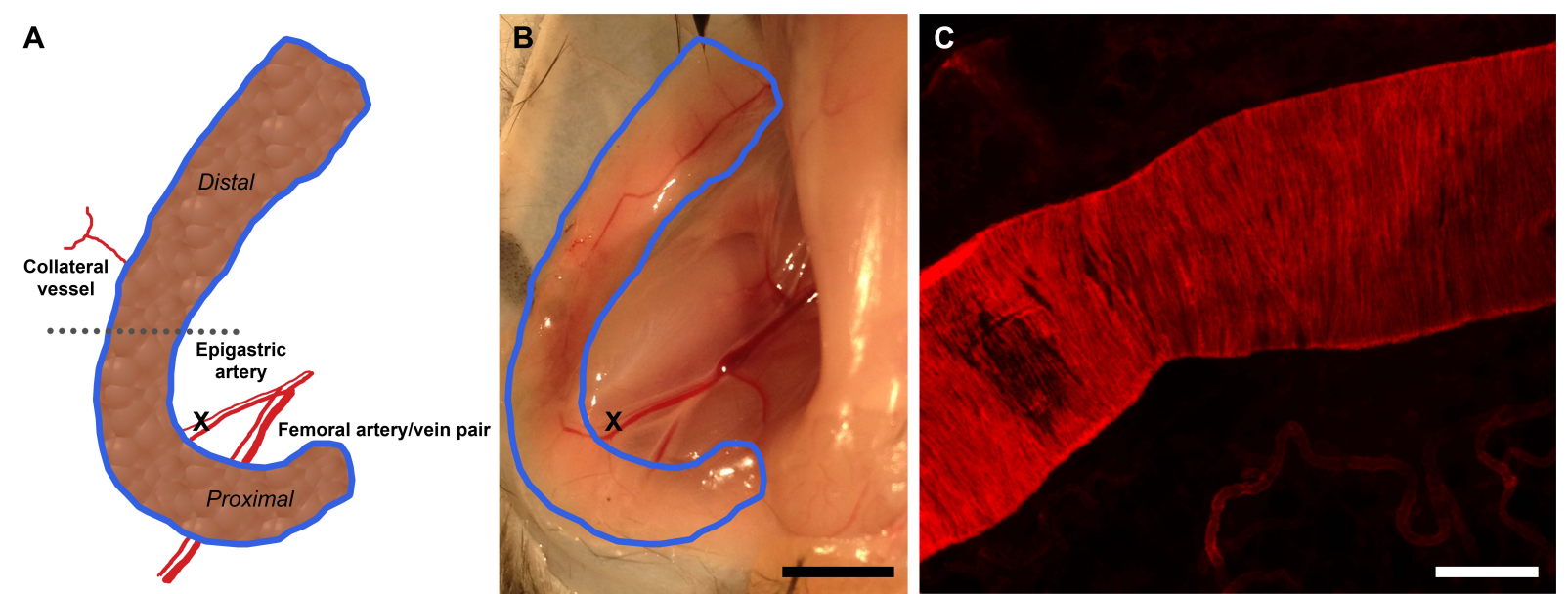

Figure 2.1: In vivo murine model of flap delay

A.) Schematic of inguinal fat pad including epigastric artery (ligation site), femoral artery, collateral vessel, and areas of interest. B.) Macroscopic view of inguinal fat pad (outlined in blue) and ligation site. Scale bar $=5 \mathrm{~mm}$. C.) Confocal micrograph of epigastric artery stained with $\alpha$-smooth muscle actin. Scale bar $=100 \mu \mathrm{m}$.

\section{Intravascular perfusion of isolectin to visualize blood flow}

The presence of flow in blood vessels was determined using intravascular perfusion of isolectin in Tie2-GFP reporter mice. Following ligation or sham surgery at specified time points (30 minutes, 12 hours, 24 hours, and 72 hours), the jugular vein was surgically exposed in anesthetized mice. $50 \mu \mathrm{g}$ of Alexa Fluor 647 conjugated Isolectin GS-IB 4 from Griffonia simplicifolia (Life Technologies, Grand Island, NY) was suspended in $150 \mu \mathrm{L}$ of sterile PBS (Life Technologies, Grand Island, NY) and drawn into a 28-gauge insulin syringe (Smiths Medical, Dublin, $\mathrm{OH})$. The needle was carefully inserted into the jugular vein and the isolectin solution was slowly injected to prevent vessel rupture. Isolectin was allowed to circulate for 10 minutes, and then the mouse was humanely euthanized via $\mathrm{CO}_{2}$ 
asphyxiation. Samples of the inguinal fat pad were harvested and whole-mounted for confocal microscopy, as described below.

\section{Harvest of adipose tissue}

Mice were euthanized via $\mathrm{CO}_{2}$ asphyxiation. Mice were positioned supine, and all four paws were pinned to corkboard. A horizontal incision was made in the abdomen to expose the peritoneal membrane without puncturing. The skin was peeled back from the mouse to expose the subcutaneous inguinal fat pad. Areas of the inguinal fat pad or collateral vessels of interest were carefully excised. The "proximal" region was defined as the area of the inguinal fat pad immediately adjacent to where the epigastric artery enters the fat pad, the "distal" region was defined as the area adjacent to where the middle collateral vessel enters the inguinal fat pad, and the "distal" region was defined as the area of the adipose tissue furthest from where the epigastric artery enters the fat pad (Figure 2.1). Adipose tissue and collateral vessels were immunostained for confocal microscopy or used in other assays, as described below.

\section{Whole mounting and confocal imaging of excised adipose tissue}

Harvested and immunostained samples of adipose tissue were allowed to adhere to gelatin coated microscope slides for 5 minutes. Slides were sealed with coverslips in 50:50 PBS/glycerol (Sigma-Aldrich, St. Louis, MO) solution. All fluorescentlylabeled samples were imaged using a Nikon TE 2000-E2 microscope (Nikon 
instruments, Melville, NY) equipped with a Melles Griot Argon Laser System (Melles Griot Carlsbad, CA) and a Nikon D-Eclipse C1 confocal attachment. $40 \mu \mathrm{m}$ Z-stacks with $2 \mu \mathrm{m}$ step size were acquired of whole-mounted tissue to account for tissue depth. Magnification power varied from 10x magnification to $60 \mathrm{x}$ magnification and is indicated by scale bars on presented images. Multiple FOVs were acquired for each tissue, as listed for each study in the figure legend.

\section{Quantification of isolectin perfusion in adipose microvessels}

Separate images were taken for both the endogenous Tie2-GFP reporter (which labels blood vessels) and the intravascularly perfused isolectin (which labels blood vessels that have flow). 200x images (at least four unique FOVs per sample) were thresholded and converted to black and white to reduce background using Image ${ }^{159}$. Images were then skeletonized so that each blood vessel was one pixel wide to account for differences in blood vessel caliber. The percent area of black pixels covering each image was calculated, and the ratio of the percent area of lectin perfused blood vessels to the percent area of the Tie2-GFP+ blood vessels was calculated. A ratio of 1.0 is indicative of complete isolectin perfusion of the Tie2$\mathrm{GFP}^{+}$blood vessels. 


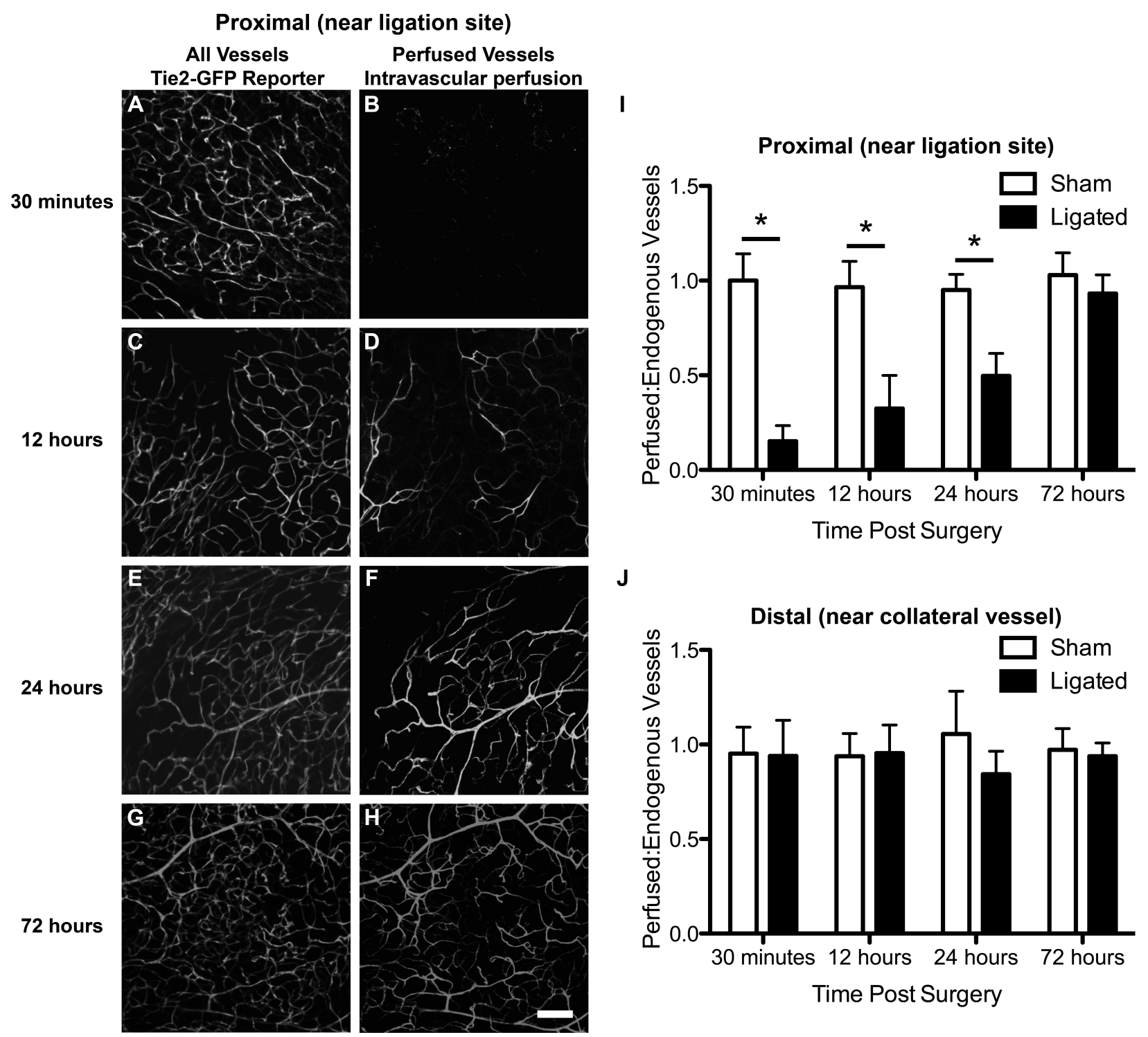

Figure 2.2: Vascular flow profile of flap delay model

Confocal micrographs reveal nearly complete flow recovery 72 hours post-ligation in the distal area. All vessels $(A, C, E, G)$ are visualized using the Tie2-GFP mouse, while perfused vessels are visualized using isolectin intravascularly perfused (B,D,F,H). $A, B$.) All vessels and perfused vessels 30 minutes post-ligation. C,D.) Endogenous and perfused vessels 12 hours post-ligation. E,F.) Endogenous vessels and perfused vessels 24 hours post-ligation. G,H.) Endogenous and perfused vessels 72 hours postligation. I.) Quantification of perfusion to all vessels in the proximal region reveals a significant decrease in perfusion in ligated tissue 30 minutes, 12 hours, and 24 hours post-surgery (p-value <0.05, Two-way ANOVA. J.) Quantification of perfusion to all vessels in the distal region reveals no significant decrease in perfusion in ligated tissue 30 minutes, 12 hours, and 24 hours post-surgery (p-value $<0.05$, Two-way ANOVA. Three mice were used for each time point and four FOVs were quantified. Scale bar $=100 \mu \mathrm{m}$. Data presented are mean + standard deviation for graphs. Microvessel network structural analysis 
Samples of adipose from ligated and sham inguinal fat pads were harvested 3 days post surgery from the specified regions (proximal or distal) and fixed overnight in $4 \%$ PFA at $4^{\circ} \mathrm{C}$. Samples were washed three times with PBS and submerged in 100 $\mu \mathrm{L}$ of $0.3 \%(\mathrm{v} / \mathrm{v})$ Triton X-100/PBS for 3 hours at room temperature to permeabilize the tissue. Following permeabilization, the tissue was submerged in $100 \mu \mathrm{L}$ of Alexa Fluor 568 conjugated isolectin diluted in $0.3 \%$ Triton X-100/PBS at 1:300. Samples were incubated in antibody solution on a rocker at $4^{\circ} \mathrm{C}$ overnight protected from light. Following staining, samples were washed five times for five minutes per wash with $0.3 \%$ Triton X-100/PBS. Samples were mounted on glass slides. Images (at least four unique FOVs per sample) were acquired using confocal microscopy. Images were acquired of regions that included only capillaries and excluded larger caliber vessels (e.g. arterioles and venules) to assess the extent of angiogenesis within the capillary bed specifically. A previously published MATLAB GUI, Rapid Analysis of Vessel Elements (RAVE) ${ }^{160}$, was used to analyze blood vessel characteristics, specifically VLD and VVF.

\section{In vitro angiogenesis sprouting assay}

Tissue was harvested 3 days post surgery from ligated and sham inguinal fat pads at the specified regions (proximal and distal) in six mice. A previously published model for determining angiogenic capability ${ }^{161}$ was used to assess the angiogenic capability of ligated and sham tissue. Briefly, six small $\sim 1 \mathrm{~mm}^{3}$ pieces of sample tissue were embedded into $40 \mu \mathrm{L}$ Growth Factor Reduced BD Matrigel Matrix 
Phenol-Red Free (BD, Franklin Lakes, NJ). After Matrigel solidified, the plug was covered with $200 \mu \mathrm{L}$ of Endothelial Cell Growth Medium (EGM-2, Lonza, Basel, Switzerland). Media was changed every other day. Four, 40x brightfield images of each adipose tissue explant were acquired using a Nikon TE 2000-E2 microscope, and the percent of explants exhibiting capillary sprouting were calculated. Explants were considered positive for sprouting when new capillary networks were observed on the periphery of the adipose tissue explant.

\section{Quantification of collateral vessel diameter}

Three days post surgery, five mice were euthanized via $\mathrm{CO}_{2}$ asphyxiation and both left and right inguinal fat pads were surgically exposed, as described above. The collateral vessel entering the fat pad was located in the distal region of the fat pad. The fascia above the collateral vessel was removed to expose the vessel. The collateral vessel was carefully removed to retain structural integrity and was placed on a gelatin-coated slide. The collateral vessel was allowed to adhere for five minutes and the collateral vessel was encircled with a hydrophobic pen. $100 \mu \mathrm{L}$ of 18.3 $\mu \mathrm{M}$ adenosine in Ringer's solution was superfused onto the vessel for five minutes to ensure vessels were maximally dilated, which allowed direct comparison between the collateral vessels of ligated and sham adipose tissues. Adenosine was aspirated, and $100 \mu \mathrm{L}$ of $4 \%$ PFA was superfused onto the vessels. Vessels were stored in a hydrated petri dish overnight at $4^{\circ} \mathrm{C}$. 
A

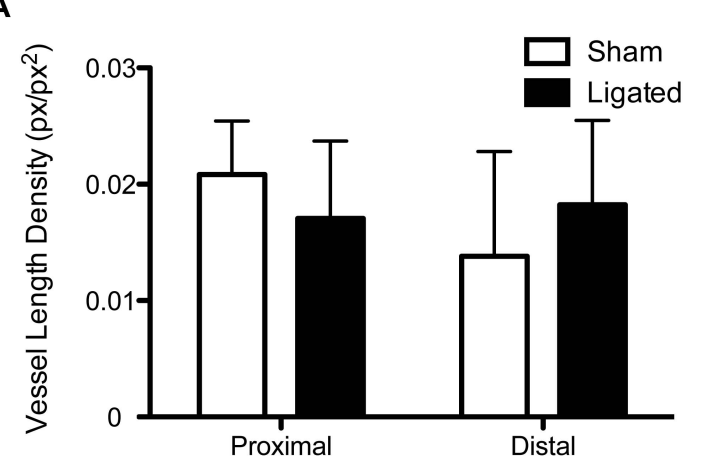

B

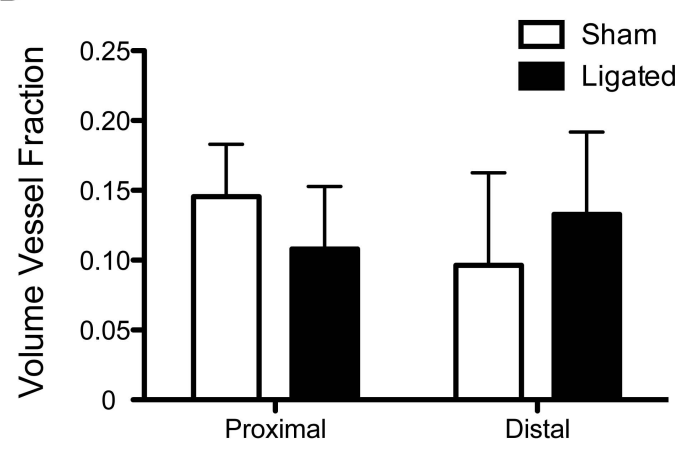

C

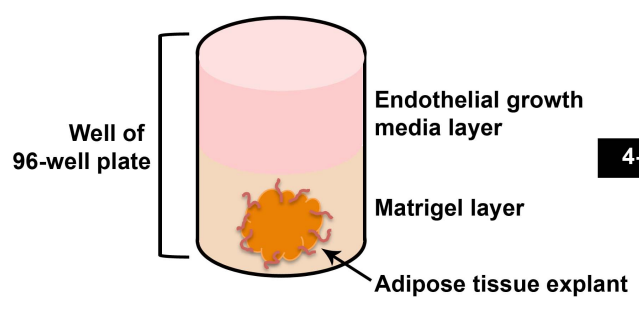

D
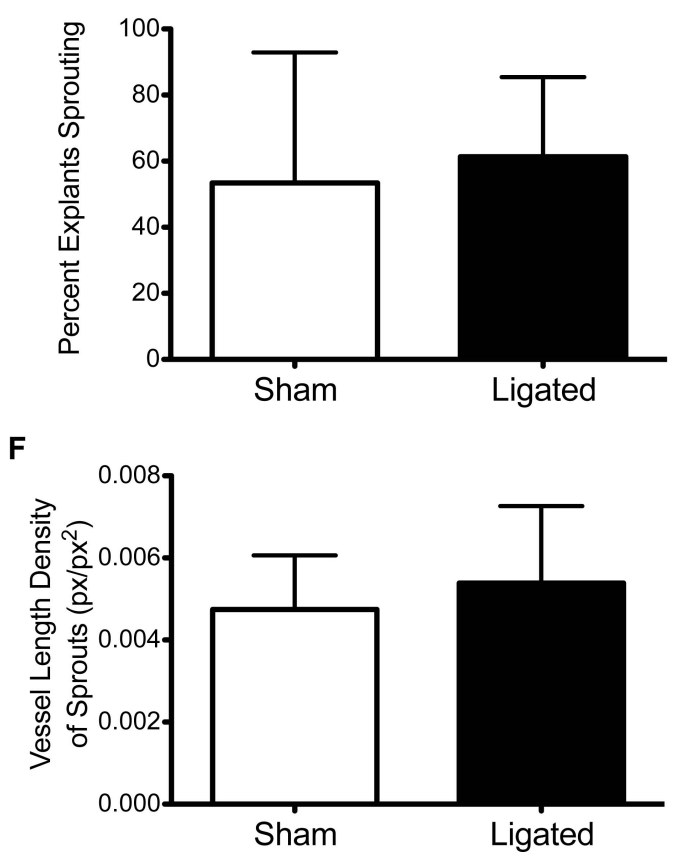

4-5 days

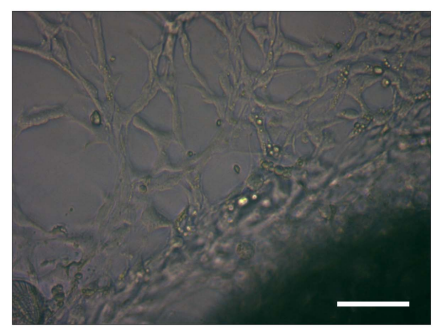

E
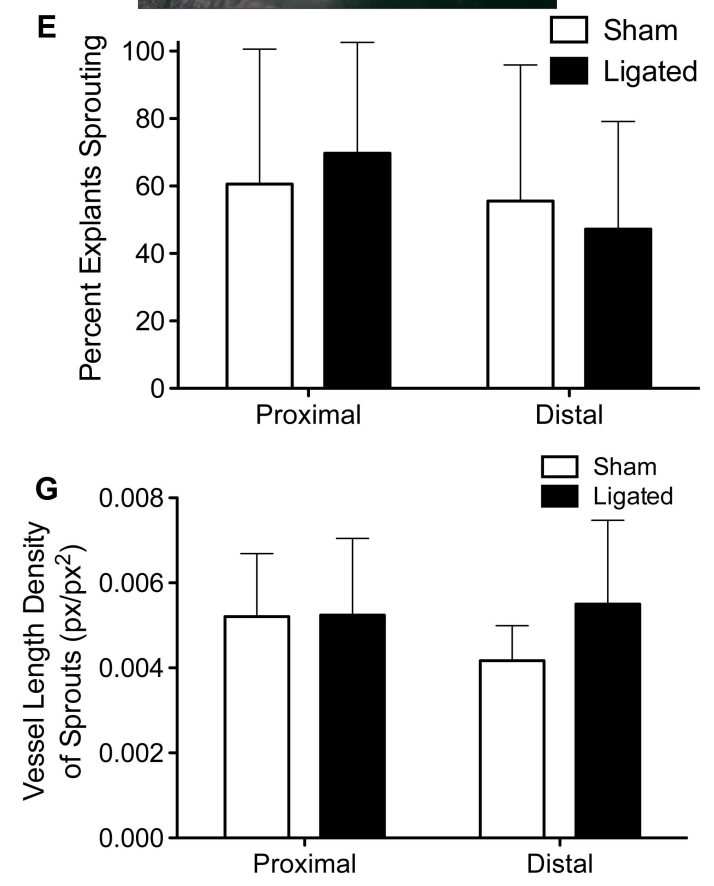

Figure 2.3: Assessment of angiogenesis in flap delay model

No difference in angiogenic capability between sham and ligated tissue. En face visualization of adipose tissue and quantification using RAVE reveals no difference in VLD (A) or VVF (B). Three mice with four FOVs were used for calculation of VLD and VVF. Schematic of angiogenic capability assay (C) to assess sprouting prevalence $(D, E)$ and vessel length density of sprouting events $(F, G)$ in sham and ligated tissue by region of interest. Six mice were used and six samples were taken from each for use in the angiogenic capability assay. Scale bar $=100 \mu \mathrm{m}$. p-value < 0.05, Two-way ANOVA. Data presented are mean + standard deviation for all graphs. 
Vessels were then immunostained to visualize the vascular smooth muscle cells, as follows. The PFA fixative solution was first aspirated and $100 \mu \mathrm{L}$ of $5 \%$ (volume/volume) mouse serum in $0.3 \%$ Triton X-100/PBS was superfused for blocking and permeabilization of the vessel for 3 hours at room temperature. The blocking and permeabilization solution was removed, and $100 \mu \mathrm{L}$ of monoclonal anti-actin, $\alpha$-smooth muscle Cy3 (Sigma-Aldrich, St. Louis, MO, Clone 1A4) diluted in $5 \%$ mouse serum in $0.3 \%$ Triton $\mathrm{X}-100 / \mathrm{PBS}$ at 1:200 was added. Samples were incubated overnight at $4^{\circ} \mathrm{C}$ protected from light. Samples were washed five times with $0.3 \%$ Triton X-100/PBS for five minutes per wash. Slides were sealed with glass coverslips in 50:50 PBS/glycerol solution.

200x images of the collateral vessels were taken using confocal microscopy. Images included the feeding branch, the first branch in the adipose tissue, and the second branch in the adipose tissue. ImageJ was used to calculate the vessel diameters. Each individual sham diameter (e.g. $1^{\text {st }}$ animal, $2^{\text {nd }}$ branch, sham) was paired with the corresponding individual ligated diameter (e.g. $1^{\text {st }}$ animal, $2^{\text {nd }}$ branch, ligated) and a paired t-test was run.

\section{CX3CR1-eGFP+ cell recruitment in inguinal fat}

Three CX3CR1-eGFP mice crossed with NG2-DsRed mice were used for the monocyte and macrophage recruitment studies ${ }^{86}$. CX3CR1-eGFP cells consist mainly of monocyte-derived macrophages, but dendritic cells and NK cells will also exhibit eGFP fluorescence ${ }^{162}$. Ligation or sham surgery was performed as described 
previously. 24 hours post-surgery, mice were anesthetized, and the jugular vein was surgically exposed. $50 \mu \mathrm{g}$ of Alexa Fluor 647 conjugated Isolectin GS-IB 4 from Griffonia simplicifoli was injected into the jugular vein and was allowed to circulate for 10 minutes, as described above. Mice were euthanized via $\mathrm{CO}_{2}$ asphyxiation. Samples of adipose were harvested at the distal location (described above) and whole-mounted for confocal imaging. 200x images (four FOVs per sample) were acquired with the collateral vessel centrally located in each image. CX3CR1-eGFP ${ }^{+}$ cells located outside of the collateral vessels were quantified per field of view using ImageJ.

\section{$C D 68^{+}$and $C D 206^{+}$macrophage quantification in inguinal fat}

Ligation or sham surgery was performed as described above. Three days postsurgery, mice were humanely euthanized and samples of adipose tissue from the proximal and distal regions of each inguinal fat pad were harvested and fixed overnight in $4 \%$ PFA at $4^{\circ} \mathrm{C}$. Samples were washed three times with PBS and submerged in $100 \mu \mathrm{L}$ of $0.3 \%(\mathrm{v} / \mathrm{v})$ Triton X-100/PBS for 3 hours at room temperature to permeabilize the tissue. Following permeabilization, the tissue was submerged in either (1) $100 \mu \mathrm{L}$ of Alexa Fluor 488 conjugated isolectin (1:300) and Alexa Fluor 647 anti-mouse CD68 (AbD Serotec, Raleigh, NC, Clone FA-11) (1:200) diluted in $0.3 \%$ Triton X-100/PBS, or (2) $100 \mu \mathrm{L}$ of Alexa Fluor 546 conjugated isolectin (1:300), Alexa Fluor 647 anti-mouse CD68 (AbD Serotec, Raleigh, NC, Clone FA-11) (1:200), and Alexa Fluor 488 anti-mouse CD206 (AbD Serotec, 
Raleigh, NC, Clone MR5D3) (1:200) diluted in 0.3\% Triton X-100/PBS. Samples were incubated in antibody solution on a rocker at $4^{\circ} \mathrm{C}$ overnight protected from light. Following staining, samples were washed five times with $0.3 \%$ Triton X100/PBS for five minutes per wash. Samples were mounted on glass slides. 200x images (at least four unique FOVs for each sample) were acquired using confocal microscopy, and $\mathrm{CD}_{68}^{+}$and $\mathrm{CD} 206^{+}$cells were quantified using ImageJ.

\section{Quantification of proliferating cells}

Samples of adipose tissue from the proximal and distal regions were acquired as described above under "Macrophage recruitment". Tissue samples were submerged in $100 \mu \mathrm{L}$ of Alexa Fluor 488 anti-mouse F4/80 (AbD Serotec, Raleigh, NC, Clone A3-1) (1:100), Alexa Fluor 546 conjugated isolectin (1:300), and Alexa Fluor 647 anti-mouse Ki-67 (Biolegend, San Diego, CA, Clone 16A8) (1:300) and diluted in 0.3\% Triton X-100/PBS. Images were acquired using confocal microscopy as described above, and $\mathrm{Ki}-67^{+}$cells, as well as $\mathrm{F} 4 / 80^{+} / \mathrm{Ki}-67^{+}$cells, were quantified in ImageJ.

\section{Statistical analysis}

A two-way ANOVA or a Student's t-test was performed, as indicated in each figure caption. Statistical significance was asserted at p-values $<0.05$. All data are presented as average + standard deviation. 


\section{Results}

Surgical ligation eliminates blood flow through the epigastric artery but perfusion is recovered 72 hours after ligation

The reproducible vascular anatomy of the inguinal fat pad (Figure 2.1A) enabled us to study vascular remodeling in both the collateral vessels and in the vessels downstream of the ligation in the epigastric artery. To study vascular growth and remodeling in different regions of the inguinal fat pad, we divided it into two regions, relative to where the epigastic artery enters the fat pad: proximal and distal (Figure 2.1A). A macroscopic view of the inguinal fat pad (Figure 2.1B) shows the femoral artery, as well as the ligation site within the epigastric artery. Immunofluorescent staining for $\alpha$-smooth muscle actin was performed to measure the diameter of the epigastric artery, which was roughly $150 \mu \mathrm{m}$ (Figure 2.1C). Loss of blood flow downstream of the ligation site in the epigastric artery was visually confirmed following ligation, and in the contralateral fat pad undisturbed blood flow was visually confirmed following sham surgery.

Use of the Tie2-GFP reporter mouse allowed for visualization of arterioles, capillaries, and venules in the inguinal fat pad. Endothelial cells in these mice express GFP under the control of an endothelial-specific receptor tyrosine kinase (Tie2) promoter. Confocal micrographs of all samples revealed the expected lacy structure of the vascular network in the adipose tissue (Figures $2.2 \mathrm{~A}, 2.2 \mathrm{C}, 2.2 \mathrm{E}$, $2.2 \mathrm{G})$. Intravascular perfusion of isolectin allowed for visualization of perfused vessels, and comparison with Tie2 expressing vessels in the same FOV (Figures $2.2 \mathrm{~B}, 2.2 \mathrm{D}, 2.2 \mathrm{~F}, 2.2 \mathrm{H})$. We hypothesized that we would see a loss in perfusion 
surrounding the ligation site (proximal) immediately following ligation, but would observe a gradual increase in perfusion due to vessel remodeling in the distal region of the inguinal fat pad. We also hypothesized that there would be little to no alteration in the perfusion of the distal blood vessels where the collateral vessel enters. Representative confocal micrographs and quantification of images such as these revealed a near complete loss of perfusion in ligated tissue in the proximal region 30 minutes post-surgery (Figure $2.2 \mathrm{~B}$ and $2.2 \mathrm{I}$ ). Gradually and linearly over the next 72 hours, ligated fat pads experienced full recovery of perfusion (Figure $2.2 \mathrm{H}$ and 2.2I). In contrast, the ratio of perfused isolectin to Tie2-GFP expressing vessels in the sham fat pad maintained a relatively constant ratio close to 1.0, indicating no loss of perfusion following sham surgery (Figure 2.2I). Additionally, examination of confocal micrographs within the distal regions of the sham and ligated tissues (images not shown) and quantification of these images (Figure 2.2J), showed no alteration in perfusion in the distal regions of either sham or ligated tissues.

Vessel length density and volume vessel fraction are not affected by arterial ligation An increased VLD and/or an increased VVF is indicative of angiogenesis. Therefore, to determine the extent of angiogenesis in the inguinal fat pad following surgical ligation of the epigastric artery, we used an automated image analysis program, RAVE to calculate VLD and VVF in samples obtained from different anatomical regions of the ligated and sham tissues. Images were acquired from isolectin 
stained, whole-mounted tissue. No significant difference in VLD or VVF was observed between ligated and sham tissue, irrespective of the anatomical region (Figure 2.3A). We also compared the VVF between ligated and sham tissue based on anatomical region and saw no significant differences (Figure 2.3B).

Angiogenic sprouting capability is not affected by ligation

To test whether ligated tissue has a higher angiogenic capability than sham tissue, we used a previously published in vitro assay of functional angiogenic capability. Small pieces of adipose tissue $\left(\sim 1-2 \mathrm{~mm}^{3}\right)$ obtained from ligated and sham tissues were embedded into growth factor reduced Matrigel supplemented with endothelial growth media (Figure 2.3C). After 4-5 days endothelial sprouting and network formation were observed (Figure 2.3C). There was no significant difference in the percent of explants that exhibited endothelial sprouting between ligated and sham tissues (Figure 2.3D) regardless of the anatomical location from which the explants were harvested (Figure 2.3E). Consistent with the en face visualization of the capillary network, we did not observe an increase in vessel length density from the sprouts observed (Figure 2.3F,G) during the angiogenic capability assay from ligated tissue when compared to sham tissue.

\section{Collateral vessel diameters enlarge following ligation}

We were able to carefully excise the distal collateral vessel and keep it structurally intact for diameter measurement under maximal dilation (Figure 2.4A). We 
removed the collateral vessel from the animal so that we could measure diameter changes in the first and second branch within the inguinal fat pad, something that is not possible using intravital microscopy due to the opacity of the adipose tissue. The measurements were taken following adenosine dilation at the feeding vessel entering the inguinal fat pad, at the first branch, and at the second branch. Relative to the distal collateral vessel on the contralateral sham side, the ligated distal collateral vessel experienced a statistically significant increase in the vessel diameter (Figure 2.4B). The average percent increase from sham to ligated tissue was $56.9 \%$ for the feeding vessel, $38.7 \%$ for the first branch, and $41.3 \%$ for the second branch.
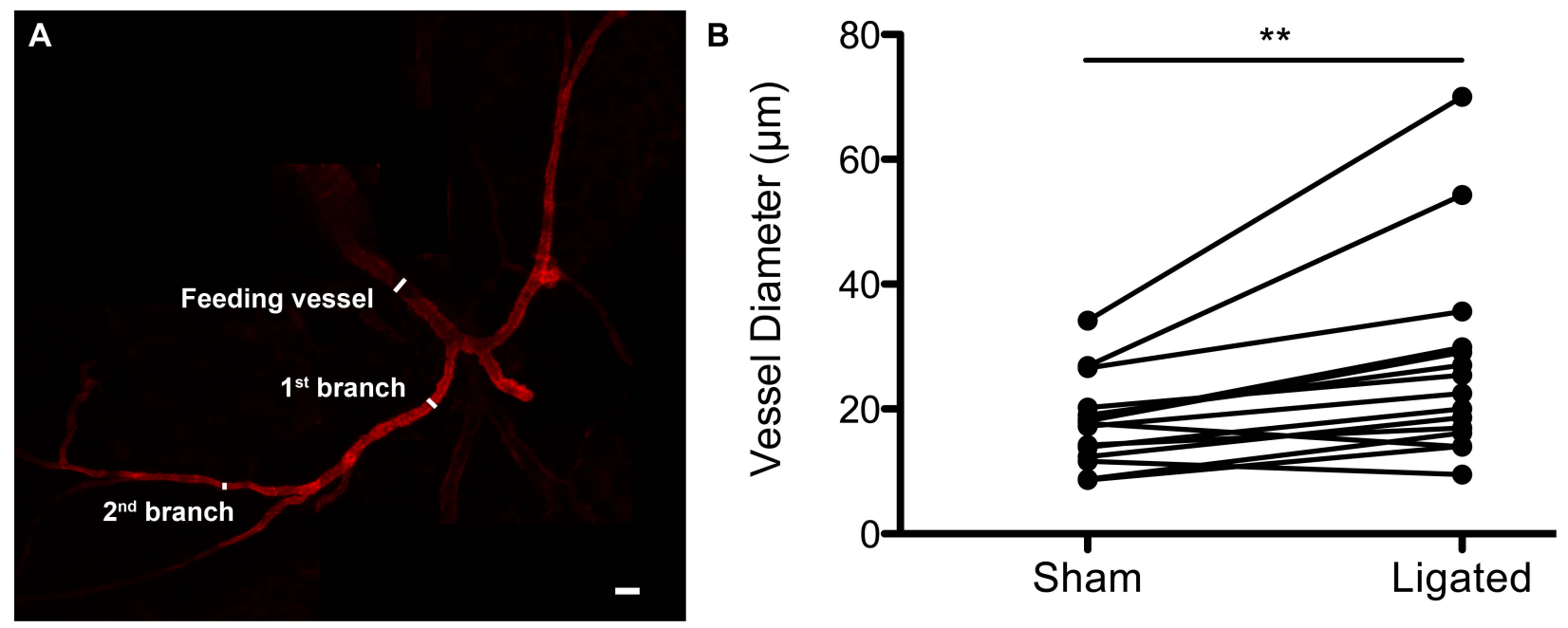

Figure 2.4: Diameter quantification of collateral vessel

Confocal micrographs of excised, structurally conserved collateral vessels reveal an increase in vessel diameter in ligated tissue when compared to paired sham tissue. A.) Excised collateral vessel stained with $\alpha$-smooth muscle actin with feeding vessel, $1^{\text {st }}$ branch, and $2^{\text {nd }}$ branch identified. B.) Individual diameter measurements for sham tissues were paired with corresponding diameter measurements for ligated tissues (in the same mouse) and a paired $t$-test was run (p-value $<0.01)$. Five mice were used to quantify collateral vessel diameters. Scale bar $=50 \mu \mathrm{m}$. 


\section{CX3CR1-eGFP monocyte recruitment is increased 24 hours post ligation}

Monocyte recruitment to remodeling arterioles is a hallmark of arteriogenesis ${ }^{163,164}$, and monocytes have been shown by our lab to extravasate from the venules as early as 6 hours post-ligation in skeletal muscle ${ }^{86}$. By using CX3CR1-eGFP mice crossed with NG2-DsRed mice and intravascularly perfusing isolectin, we were able to distinguish arterioles from venules ${ }^{55}$ and examine the time course of recruitment of monocytes $\left(\mathrm{eGFP}^{+}\right.$cells) to the remodeling tissue in the distal area of the inguinal fat pad. 24 hours after surgery, a statistically significant increase in CX3CR1$\mathrm{eGFP}^{+}$cells was seen in the ligated tissue when compared to the sham tissue (Figures 2.5A, 2.5B). Quantification revealed an average of $68 \mathrm{eGFP}^{+}$cells in the ligated tissue per FOV as compared to $10 \mathrm{eGFP}^{+}$cells in the sham tissue per FOV after 24 hours (Figure 2.5C).
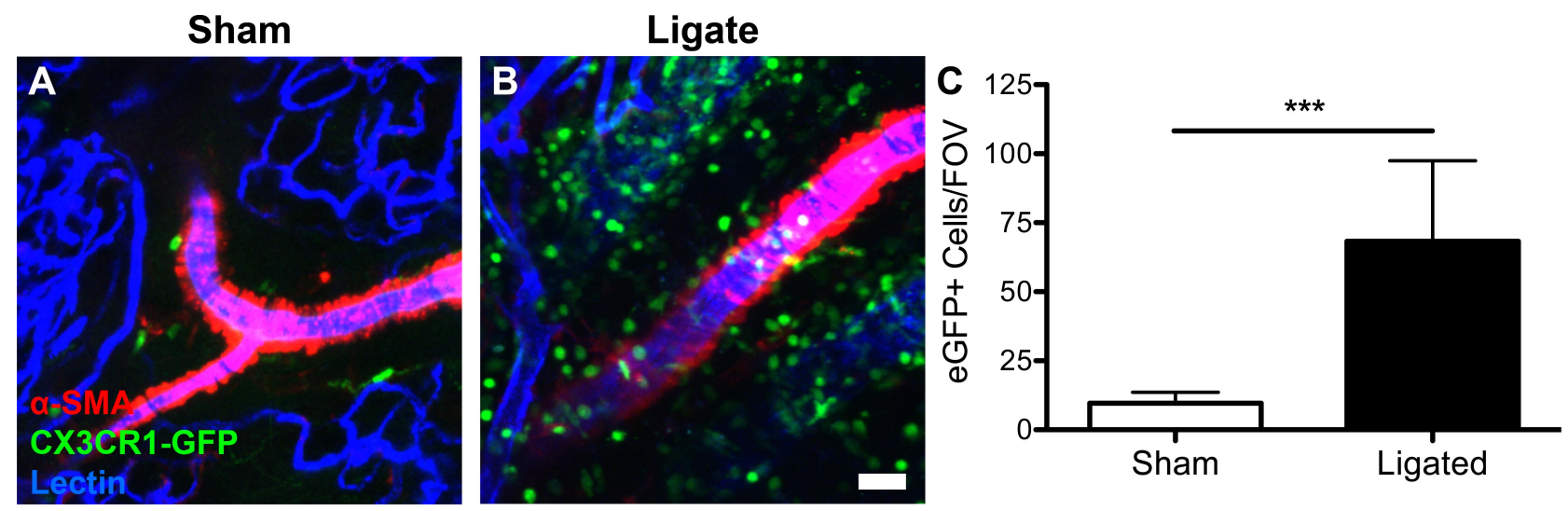

Figure 2.5: Monocyte recruitment to collateral vessel

Confocal microscopy revealed an increased presence of recruited monocytes to ligated tissue. A,B.) Confocal micrographs show an increase in CX3CR1-eGFP+ cells (green) in ligated tissue when compared to sham tissue. C.) Quantification of eGFP+ ${ }^{+}$cells per $F O V$ reveals an increase in eGFP+ cells in ligated tissue (p-value $<0.001$, Student's $t$ test). Three mice were used and four FOVs were quantified. Scale bar $=50 \mu \mathrm{m}$. Data presented are mean + standard deviation. 
$C D 68^{+}$and $C D 206^{+}$cell recruitment is increased 72 hours post ligation

72 hours post-ligation, an increase in $\mathrm{CD}^{2} 8^{+}$cells (i.e. macrophages ${ }^{165}$ ) was seen in the distal region of the ligated tissue (Figure 2.6A) when compared to the distal region of the sham tissue (Figure 2.6B). Quantification of the number of $\mathrm{CD}^{+} 8^{+}$cells per FOV revealed that there were roughly twice as many $\mathrm{CD} 68^{+}$cells per FOV in the ligated distal tissue $\left(113 \mathrm{CD}^{+} 8^{+}\right.$cells/FOV) as compared to the sham distal tissue (66 CD68 ${ }^{+}$cells/FOV) (Figure 2.6C). There was no significant difference in the number of $\mathrm{CD}^{+} 8^{+}$cells in either the proximal region of the ligated tissue or the proximal region of the sham tissue (Figure 2.6C).
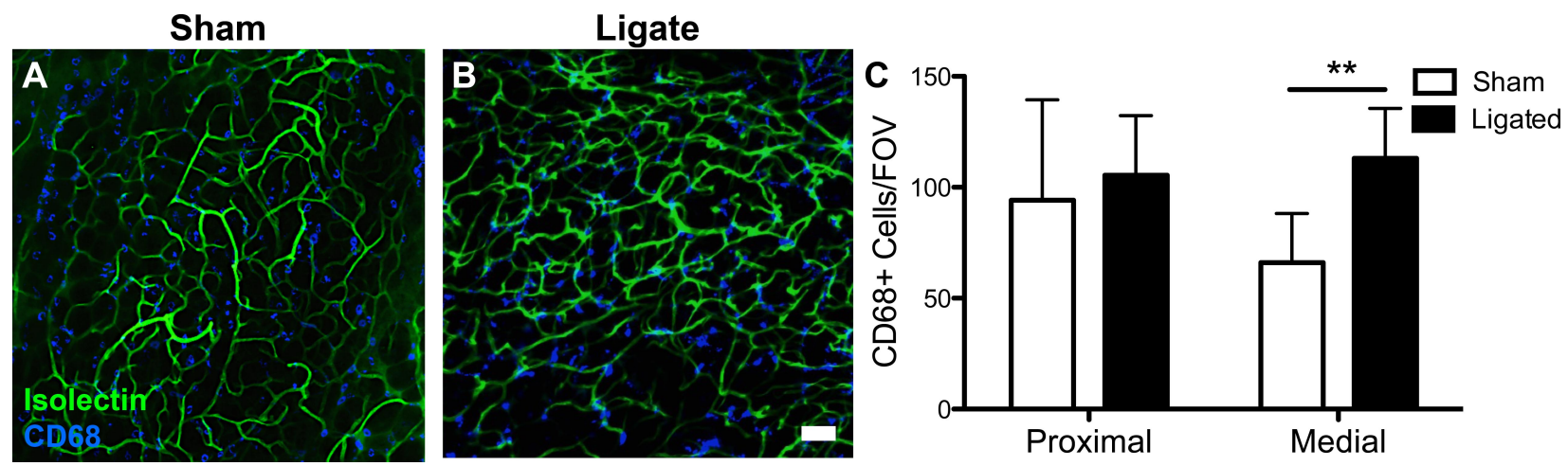

Figure 2.6: Macrophage recruitment to collateral vessel

Immunofluorescent staining revealed an increase in the number of macrophages within ligated tissue. A,B.) Confocal micrographs show an increase in CD68+ cells (blue) in ligated tissue when compared to sham tissue. C.) Quantification of $\mathrm{CD}^{6} 8^{+}$ cells per FOV reveals an increase in $\mathrm{CD} 68^{+}$cells in ligated tissue in the distal region (p-value<0.01, Two-way ANOVA). Three mice were used and four FOVs were quantified. Three mice were used and four FOVs were quantified. Scale bar $=50 \mathrm{\mu m}$. Data presented are mean + standard deviation.

72 hours post-ligation, we observed an increase in the number of $\mathrm{CD}^{+} / \mathrm{CD}^{206} 6^{+}$cells in the distal tissue when compared to the sham distal tissue (Figures 2.7A, 2.7B). We used CD206, the murine mannose receptor, coupled with the CD68 antibody to identify M2 polarized macrophages based on previous 
literature and studies $86,166-168$. Interestingly, the shape, size, and location of these CD68+/CD206 ${ }^{+}$cells were markedly different in ligated as compared to sham tissue. In ligated tissues, $\mathrm{CD} 68^{+} / \mathrm{CD} 206^{+}$cells were much larger, as compared to the $\mathrm{CD}^{+} / \mathrm{CD} 206^{+}$cells in sham tissue (Figure $2.7 \mathrm{~A}, \mathrm{~B}$ ). Moreover, CD68 ${ }^{+} / \mathrm{CD} 206^{+}$cells in sham tissues were located on vessels, appearing to wrap around the capillaries, but in ligated tissues $\mathrm{CD} 68^{+} / \mathrm{CD} 206^{+}$cells did not appear to be associated with or adjacent to blood vessels in this manner. Further, the $\mathrm{CD} 68^{+} / \mathrm{CD} 206^{+}$cells in the ligated tissue were frequently present in pairs, which was an infrequent observation in sham tissue.
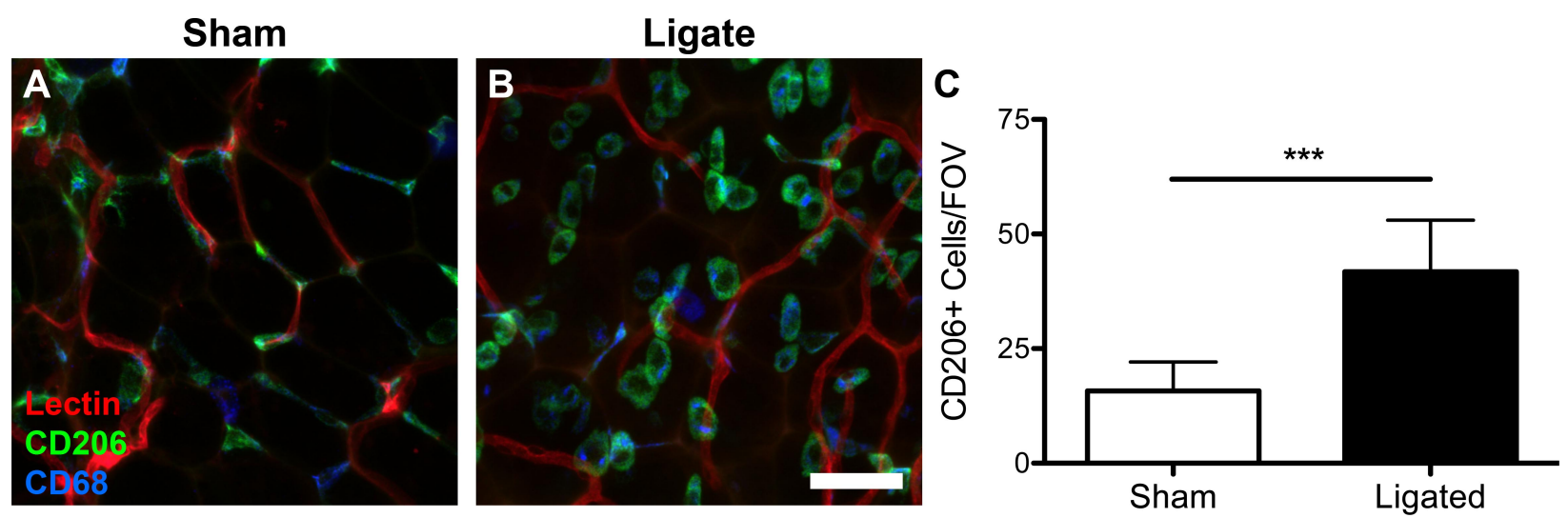

Figure 2.7: Anti-inflammatory macrophage recruitment to collateral vessel

More anti-inflammatory macrophages were found in ligated tissue. A,B.) Confocal micrographs show an increase in $\mathrm{CD}^{206^{+}}$cells (green) in ligated tissue when compared to sham tissue. C.) Quantification of $\mathrm{CD}^{206^{+}}$cells per FOV reveals an increase in $\mathrm{CD}^{206^{+}}$cells in ligated tissue in the distal region (p-value $<0.001$, Student's t-test). Three mice were used and four FOVs were quantified. Scale bar = $50 \mu \mathrm{m}$. Data presented are mean + standard deviation.

Ki- $67^{+}$cells are increased in ligated tissue

To evaluate the number of proliferating cells, a cellular marker for entry into the active phases, Ki-67, was used. The Ki-67 protein is absent when cells are resting 
$\left(\mathrm{G}_{0}\right)$ and present when cells are in active phases $\left(\mathrm{G}_{1}, \mathrm{~S}, \mathrm{G}_{2}\right.$, and mitosis). Seventytwo hours post-ligation, we observed an increase in Ki- $67^{+}$cells in the distal region of the ligated tissue (Figure 2.8A) when compared to the distal region of the sham tissue (Figure 2.8B). Quantification revealed roughly six times more Ki-67+ cells in the ligated distal tissue (average $39 \mathrm{Ki}-67^{+}$cells) than in the sham ligated tissue (average $175 \mathrm{Ki}-67^{+}$cells) (Figure $2.8 \mathrm{C}$ ).
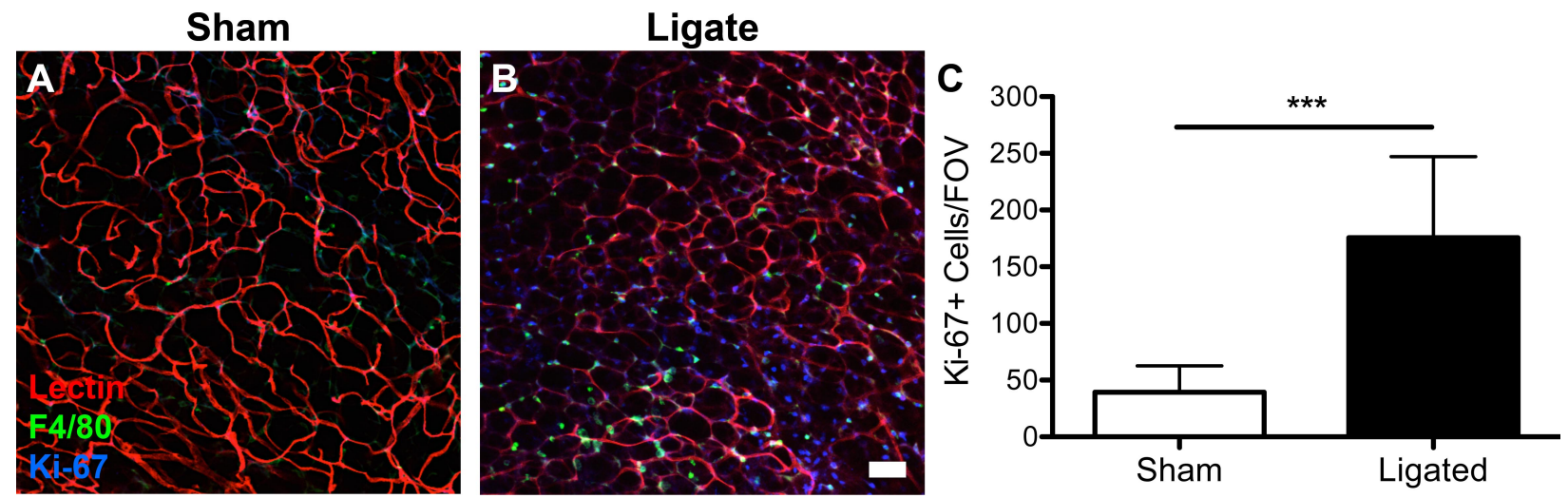

Figure 2.8: Proliferating cells surrounding collateral vessel

Cell proliferation was increased in ligated tissue. A,B.) Confocal micrographs show an increase in $\mathrm{Ki}-67^{+}$cells (blue) in ligated tissue when compared to sham tissue. C.) Quantification of $\mathrm{Ki}-67^{+}$cells per $\mathrm{FOV}$ reveals an increase in $\mathrm{Ki}-67^{+}$cells in ligated tissue in the distal region (p-value $<0.001$, Student's $t$-test). Three mice were used and four FOVs were quantified. Scale bar $=50 \mu \mathrm{m}$. Data presented are mean + standard deviation.

Co-labeling of excised ligated and sham fat pads with F4/80 and Ki-67 revealed enhanced co-localization of F4/80 and Ki-67 in ligated tissues compared to sham tissues, suggesting that macrophage proliferation is/was occurring. There were, on average, $63 \mathrm{~F} 4 / 80^{+} / \mathrm{Ki}-67^{+}$cells in ligated tissue per FOV with $45.4 \%$ of F4/80 ${ }^{+}$cells also staining positively for Ki-67. In sham tissue, there were, on average, $8 \mathrm{~F} 4 / 80^{+} / \mathrm{Ki}-67^{+}$cells per $\mathrm{FOV}$ with $13.1 \%$ of $\mathrm{F} 4 / 80^{+}$cells also staining positively for Ki-67. Co-localization of F4/80 and Ki-67, coupled with the timing of 
the $\mathrm{CD}^{+}{ }^{+} / \mathrm{CD} 206^{+}$recruitment to ligated tissue (72 hours), agrees with the notion that M2 macrophages are being recruited to ligated tissue where they are proliferating. Co-labeling with $\mathrm{CD}^{+} / \mathrm{CD} 206^{+}$(Figure 2.7B) revealed enlarged, paired M2 macrophages, which may also be indicative of active cell proliferation. 


\section{Discussion}

Microvascular growth and remodeling in response to altered blood flow is an important adaptive response that has been widely studied in heart ${ }^{164}$, skeletal muscle $^{86,151,169}$, and brain ${ }^{170}$, among other organs. Far less is known about these processes in adipose tissue, despite the fact that the body's response to altered blood flow in skin, fat, and muscle is currently exploited in the clinical use of flap delay. The goal of our study was to examine structural adaptations of the microvasculature - at the network level - in response to altered blood flow in the murine inguinal fat pad. By using a combination of in vitro and in vivo assays, we found that arteriogenesis, not angiogenesis, predominates in adipose three days after surgical ligation of the main feeding artery. Further, we found that CX3CR1 ${ }^{+}$ monocytes are actively recruited during collateral vessel diameter enlargement in fat. Localized proliferation of $\mathrm{F} 4 / 80^{+}$cells around remodeling collateral arterioles in the inguinal fat pad 72 hours after ligation was synchronized with the observed increase in the number of $\mathrm{CD} 68^{+} / \mathrm{CD} 206^{+}$cells, suggesting a potential role for macrophages in collateral vessel remodeling, as has been observed in other tissues $86,87,93,149$.

Mechanisms of collateral vessel remodeling have been widely studied in skeletal muscle in the context of peripheral artery disease ${ }^{171,172}$. Briefly, it has been suggested that obstruction in blood flow causes a redistribution of blood flow through collateral vessels leading to alterations in the wall shear stress levels experienced by the vascular endothelium, which increases cytokine (e.g. monocyte chemotactic protein-1) production by endothelial cells ${ }^{94,173}$. Hypoxia and/or ischemia 
may also contribute to collateral vessel enlargement, as epicardial collateral vessel enlargement in the coronary circulation has been shown to occur in the absence of pressure gradients or wall shear stress changes following partial embolization in coronary arterioles ${ }^{174}$. Recruitment and activation of monocytes downstream of ischemia and/or hypoxia is an important process in collateral vessel enlargement. Our lab has recently shown that in murine skeletal muscle, monocytes extravasate from venules that are paired with collateral arterioles following ligation. Recruitment of monocytes, followed by elevated levels of $\mathrm{CD}^{+} / 8^{+} \mathrm{CD} 206^{+}$ macrophages, was associated with vascular smooth muscle cell proliferation in collateral arterioles and collateral vessel diameter expansion by 72 hours $^{86}$. Our data collected in adipose tissue are consistent with these observations in skeletal muscle that implicate monocytes and monocyte-derived macrophages in collateral arteriole diameter expansion ${ }^{86}$. Here, we measured an increase in monocyte recruitment to the remodeling arteriole 24 hours post-ligation. We also observed a significant increase in $\mathrm{CD}^{+} 8^{+}$macrophages and in $\mathrm{CD} 68^{+} / \mathrm{CD} 206^{+}$macrophages in the ligated tissue when compared to the sham tissue three days post-surgery. This was accompanied by an increase in $\mathrm{Ki}-67^{+}$cells that were $\mathrm{F} 4 / 80^{+}$. Approximately $45 \%$ of $\mathrm{F} 4 / 80^{+}$cells also stained for Ki-67 in ligated tissue as compared to $13 \%$ of $\mathrm{F} 4 / 80^{+}$cells in sham tissue staining positive for Ki-67. Together, our findings suggest that monocyte-derived macrophages recruited from the circulation and proliferation of macrophages in the tissue play important roles in collateral vessel remodeling in adipose tissue. Whether or not $\mathrm{F} 4 / 80^{+}$macrophages originated from 
recruited, monocyte-derived $\mathrm{CX} 3 \mathrm{CR} 1^{+}$cells and the extent to which these recruited cells overlap the $\mathrm{CD} 68^{+} / \mathrm{CD} 206^{+}$macrophage population was not evaluated by our study. It is notable, however, that the observations we present here in murine adipose tissue mimic what has previously been observed in murine skeletal muscle $^{86}$. We suggest that parallels in the time course of collateral vessel remodeling in muscle and fat may be related to the fact that the time course of the murine immune response and the diameters of the collateral vessels in both tissues are relatively equivalent, even though the metabolic demand between these tissue beds may differ substantially ${ }^{175}$.

In our study, we observed diameter increase in the collateral arterioles that were consistent with previous studies involving the ligation of a feeder artery ${ }^{102,151}$. Moreover, we found that arteriogenesis - the diameter expansion of existing collateral arterioles due to structural remodeling of the vessel wall - predominated over angiogenesis in adipose tissue following surgical ligation of the main feeding artery to the inguinal fat pad. The initiating stimulus in our model is still in question; we did not determine the extent to which hypoxia vs. wall shear stress changes contributed to the observed collateral enlargement in this model. In future work, it will be informative to measure wall shear stress in the collateral arterioles that experienced diameter enlargements, and to evaluate whether or not the adipose tissue becomes hypoxic after ligation.

While the role of angiogenesis in response to skeletal muscle ischemia appears to be essential, both in pre-clinical models and in clinical data 
studies $^{93,146,176,177}$, the role of angiogenesis in vessel adaptations to ischemia in adipose tissue remains unclear. Indeed, there is a dichotomy of findings from previous studies examining the role of angiogenesis and new blood vessel growth in adipose tissue - some studies cite an increase in angiogenesis, while others suggest no change or even a decrease ${ }^{158,178}$. Our studies in the murine inguinal fat pad agree with the latter - angiogenesis is not a major component of the adipose tissue's response to arterial ligation, at least within the first three days following ligation. We confirmed this finding with in vitro assays as well as en face visualization of the vascular network within the adipose tissue. It is possible that we did not observe an increase in angiogenesis because the collateral vessel's ability to rapidly remodel (three days) obviates the need for new capillary growth. Perhaps inducing a more severe degree of ischemia in the inguinal fat pad by ligating the femoral artery, similar to Suga et al. ${ }^{147}$ (ligation of all collateral blood vessels), would provide a stimuli more inductive of angiogenesis. Adipose tissue has been shown to be tolerant of hypoxia (as evidenced by viable adipocytes in hypoxic, obese adipose tissue $^{179}$ ) and has a lower resting metabolic demand when compared to skeletal muscle ${ }^{175}$. Both of these factors may mitigate the need for new capillary growth into adipose tissue, and may partially explain why we did not observe angiogenesis in our ligation model.

Our goal was to study the microvascular remodeling responses to vessel ligation in adipose tissue in order to better understand why there are improved clinical surgical outcomes associated with flap delay. However, our use of a murine 
model of flap delay makes several assumptions and oversimplifies the clinical flap delay procedure. For example, the composition and size of the flap that we examined was different than most surgical flaps used in clinical reconstructive surgery. While some surgical flaps consist solely of adipose tissue, most flaps are a composite tissue consisting of adipose tissue, muscle, and skin ${ }^{19}$. While our murine model is a simplification of the clinical scenario, we feel that our study can offer valuable insight because the adipose tissue component of flaps is the most voluminous part of the flap and is usually the main determinant of overall volume retention. Specifically, breast reconstructive flaps often consist entirely of adipose tissue and only a small amount of skin. We analyzed the remodeling response in the flap 1-3 days post-surgery rather than 10-14 days post-surgery as is practiced clinically and in previously published pre-clinical in vivo models ${ }^{157,158}$ used to investigate flap delay. We chose earlier time points for our studies because we were interested in observing the transient recruitment of monocytes and macrophage differentiation (0-3 days) during the remodeling response that happen much sooner than the 10-14 days, which is the clinical time frame for flap delay. Notably, the size of the "flap" we used in our murine model was much smaller relative to the flaps that are preconditioned with flap delay procedures in humans. The smaller size of our murine "flap" may protect it from oxygen diffusion limitations, and this may explain why we did not see a potent induction of angiogenesis in our studies. Moreover, it has been shown that the murine immune system differs quite substantially from the human immune system ${ }^{180}$; therefore, the analogous 
macrophage subpopulations in humans may be different from those studied here in mice. It may be possible to address some of the limitations of our study by performing future work in rat or rabbit models. We chose to use a published murine model because we made use of cell-type specific fluorescent reporters, which is difficult in rats or rabbits. However duplicating our study in a larger, more complex flap, such as the transverse rectus abdominis muscle flap ${ }^{178,158}$, would allow for a better comparison to human flaps. Our study motivates future work that should examine how the enhancement and/or depletion of monocytes and macrophages affect collateral vessel remodeling in adipose tissue. Although our current studies focused on monocyte and macrophage recruitment to the remodeling adipose tissue, examining other cell types ( $\mathrm{T}$ cells, B cells, dendritic cells and other monocyte subsets ${ }^{106}$ ) within the adipose tissue would be of value for future studies.

We feel that our studies add to the previous studies that examined flap delay in various pre-clinical models. While our animal model does not equate perfectly to the human anatomy that a plastic surgeon encounters when performing the flap delay procedure in the clinic, we believe that our findings highlight the importance of collateral vessel enlargement (arteriogenesis) over new vessel sprouting (angiogenesis). Our studies provide evidence that the remodeling response may initiate much sooner (within 1-3 days) than previously observed in other pre-clinical models ${ }^{157,158}$ and clinically. This finding may be of potential interest to surgeons that are forced by clinical circumstance to perform the flap transfer procedure earlier than 14 days, as there may already be potential benefit from flap delay 
procedure at 1-3 days. We also provide a possible therapeutic mechanism (recruitment of anti-inflammatory macrophages) that may be targetable by pharmacological means ${ }^{138}$.

A surgeon will evaluate several metrics of the flap prior to deciding which artery to ligate for the flap delay procedure. Based on the volume of the tissue that needs to be transferred, a surgeon will perform a more aggressive or less aggressive flap delay procedure. For very large volume flaps ( 600-800 grams), a surgeon will try to ligate most of the feeding arterioles, but for small volume flaps ( 200-300 grams) a surgeon will ligate only a few feeding arteries into the flap. The implicit rationale for this practice is that the extent of microvascular remodeling will be proportional to the extent of arterial ligation, and larger flaps will require more microvascular remodeling and hence more extensive ligations. However, the doseresponse relationship between number of ligations and resulting arteriogenesis has not been explored. Although we do not directly address this in our study, our study shows that ligating even a single feeding artery can cause dramatic diameter expansion in smaller diameter collateral arterioles (e.g. 50 to 100 micrometers in diameter). Further, our data suggest that it is important to consider the remodeling responsiveness of smaller diameter arterioles that are capable of undergoing diameter enlargement. This is a particularly important finding, given the fact that in the clinical setting, these vessels are invisible to the surgeon and too small to be ligated but may be important contributors to the clinical benefit of flap delay. 
In summary, our study closely examined the microvascular adaptations that occur in adipose tissue following ligation of a major feeding artery and provides potential cell targets (i.e. monocytes and macrophages) to enhance blood flow within delayed flaps. Understanding how the microvasculature of adipose tissue structurally adapts in response to surgically-induced ligation can suggest therapeutic mechanisms to further enhance flap survival following flap delay and may even eliminate the need for this pre-reconstructive surgical technique leading to fewer corrective procedures and reduced cost burden on the healthcare system. 


\section{Conclusions}

Autologous adipose tissue grafting is being increasingly used to correct soft tissue trauma defects, congenital disorders, and disease resections, but resorption of grafted adipose tissue is high and unpredictable due to supposed improper vascularization. This study examines microvascular remodeling and macrophage recruitment in adipose tissue following ligation of the epigastric artery feeding the inguinal fat pad. We show that macrophage recruitment and synchronized arteriogenesis are responsible for microvascular changes within the adipose tissue. While macrophage polarization and collateral diameter expansion/arteriogenesis following induced ischemia have been studied extensively in skeletal muscle, we are the first to study these processes in adipose. We hope that this paper will serve as a platform for new studies examining microvascular remodeling within adipose tissue and ultimately improve grafted adipose tissue retention rates. 


\section{Chapter 3}

\section{COLLATERAL VESSEL REMODELING IN MURINE ADIPOSE TISSUE IS CONTINGENT ON MACROPHAGE POPULATIONS}




\section{Abstract \\ Objective}

A common surgical pre-treatment strategy, "flap delay", is a technique employed by plastic surgeons prior to autologous flap transplantation in which the main feeding artery into an adipose flap is ligated 10-14 days prior to transfer. By using a previously published murine flap delay model, we observed that the alteration in blood flow into the flap induces microvascular changes, namely arteriogenesis accompanied with a local increase in M2 macrophages. Here, we aim to test our hypothesis that recruited M2 macrophages are required for this arteriogenic enhancement within the flap by performing gain-of-function and loss-of-function studies.

\section{Methods and Results}

We used our previously published murine model of flap delay to study the effects of locally depleting and enhancing the macrophage population within the adipose flap. For gain-of-function studies, we (1) directly injected M2 differentiated macrophages into the flap and (2) applied a thin biomaterial film loaded with a pharmacological agent (FTY720) that has been previously shown to preferentially recruit M2 macrophages to remodeling tissue. For loss-of-function studies, we depleted local macrophage populations within the inguinal fat pad by clodronate liposome delivery. All interventions were applied to the fat pad during surgery, and collateral vessel diameter enlargement (arteriogenesis) and macrophage numbers within the tissue were quantified. We observed an increase in the collateral vessel diameter after ligation surgery for fat pads directly supplemented with M2 macrophages and 
for fat pads with FTY720-loaded films. Concurrently, we observed an increase in CD206 $^{+}$cells in M2 supplemented tissues and FTY720 treated tissues. Clodronate liposomes decreased the number of CD68+ cells and decreased the collateral vessel diameter measurements after ligation surgery. Taken together, these data suggest that macrophages, and specifically M2 macrophages, are important in the arteriogenesis remodeling response observed in our murine flap delay model.

\section{Conclusions}

This study underscores the importance of M2 macrophages during microvascular adaptations (mainly arteriogenesis) induced by flap delay. These studies suggest a mechanism for a translatable therapeutic target that may be used to enhance the flap delay procedure. 


\section{Introduction}

Microvascular growth and remodeling is an adaptive process that has been widely studied in skeletal muscle $86,151,169$, heart ${ }^{164}$, and brain ${ }^{170}$, among many other organs. Arteriogenesis and angiogenesis represent two compensatory mechanisms that are crucial to restore proper blood flow and maintain tissue health in response to biomechanical/biochemical cues (i.e. hypoxia or increased shear stress). Arteriogenesis is the process by which collateral vessel increase their diameter $68,95,149$ while angiogenesis is the formation of new blood vessels from preexisting blood vessels ${ }^{146-148}$. Far less is known about these microvascular remodeling responses in adipose tissue despite plastic surgeons harnessing these innate microvascular adaptations to improve autologous fat grafting retention rates by using a pre-surgery technique called "flap delay"155,156. Flap delay involves the ligation of the main vascular pedicle into the flap 10-14 days prior to tissue transfer. It is believed that the induction of altered blood flow into the flap elicits a remodeling response within the flap (increased vascular growth, for example), which leads to better graft survival. In our preceding studies, we adapted a preexisting model ${ }^{147}$ to formulate a clinically relevant murine model of flap delay ${ }^{181}$. Our findings in Chapter 2 suggest that recruitment and proliferation of antiinflammatory M2 macrophages and subsequent enlargement of a pre-existing collateral vessel (arteriogenesis) is the main mechanism responsible for the observed microvascular changes within the flap ${ }^{181}$. In order to better understand the proposed mechanism responsible during flap delay, we conducted a series of gain-of-function and loss-of-function studies in this pre-clinical model of flap delay, 
harnessing the published capabilities of the following interventions: (1) exogenous macrophage delivery (gain-of-function), (2) treatment with sphingosine-1-phosphate receptor agonist (gain-of-function), and (3) clodronate liposome delivery (loss-offunction).

Macrophages are relatively easy to isolate from mouse bone marrow or the mouse peritoneal cavity and their accessibility facilitates the study of macrophage phenotypes and function in vitro ${ }^{135,182,183}$. Subsequent polarization of isolated macrophages into M1 or M2 phenotypes is a straightforward process made possible by cytokine media supplementation, such as IL-4 for M2 macrophages and LPS or IFN $\gamma$ for M1 macrophages ${ }^{135,184,185}$. Previous studies have fluorescently labeled harvested macrophages with dyes and MRI contrast agents in vitro ${ }^{186,187}$ and reintroduced these labeled macrophages intravenously ${ }^{186}$ or via direct injection ${ }^{187-}$ 189 into various models/tissue to study effects of macrophages on obesity ${ }^{186}$, myocardial infarctions ${ }^{187}$, and hindlimb ischemia ${ }^{188}$. Labeling macrophages prior to injection allows them to be tracked to the tissue of interest, where they may contribute to the observed healing and regenerative outcomes. Based on these previous studies, we supplemented inguinal fat pads after surgical ligation of a feeding arteriole with labeled, in vitro M2-differentiated macrophages to determine the effect of macrophages on the arteriogenic response that we documented in the studies reported in Chapter 2.

Sphingosine-1-phosphate (S1P) is a short-lived bioactive lipid that serves as a ligand to five $\mathrm{G}$ protein-coupled receptors $\left(\mathrm{S}_{\mathrm{P}} \mathrm{P}_{1-5}\right)^{190,191}$. These five receptors are 
ubiquitously expressed and regulate many downstream signals, which allows the regulation of many physiological processes ${ }^{190}$ including angiogenesis and vascular maturation ${ }^{192-194}$, arteriogenesis ${ }^{138,195}$, heart development ${ }^{196}$, and immunity ${ }^{197-199}$. FTY720 (fingolimod), a $\mathrm{S}_{1} \mathrm{P}_{1,3}$ agonist, is an FDA approved drug that is currently used to treat flare-ups of multiple sclerosis ${ }^{200}$. Recent studies conducted in collaboration with our group has found that application of an FTY720-loaded PLAGA film lead to increased anti-inflammatory monocyte/macrophage recruitment ${ }^{138}$ as well as an increase in arteriogenesis in skeletal muscle ${ }^{195}$. In these studies we use the application of an FTY720-loaded film as a gain-of-function intervention to study the recruitment of M2 macrophages and subsequent arteriogenic effects.

Clodronate liposomes provide a means to transiently and locally deplete macrophage populations within a tissue of interest ${ }^{201}$. Clodronate liposomes are sub-micron spheres that consist of a drug, dichloromethylene-bisphosphonate (CI2MBP or clodronate), as cargo encapsulated within a phospholipid bilayer "shell"201,202. Upon presentation to phagocytic cells (macrophages), the liposomes are readily ingested and clodronate is released into the intracellular space from the liposomes, which causes apoptosis by the phagocytic cell ${ }^{202}$. A substantial number of studies conducted in various tissues (skeletal muscle ${ }^{203}$, tendon ${ }^{204}$, kidney ${ }^{205}$ ) have successfully utilized clodronate liposomes to transiently deplete macrophages and study this depletion effect locally. In this study, we use injections of clodronate- 
containing liposomes in our pre-clinical model of flap delay as a means to locally deplete macrophage populations within the inguinal fat pad.

In summary, we aimed to further investigate the mechanisms underpinning vascular adaptations in adipose tissue that we documented in the studies reported in Chapter 2 by testing the hypothesis that the recruitment of anti-inflammatory (M2) macrophages is necessary for arteriogenesis following the flap delay procedure. Our gain-of-function studies successfully increased anti-inflammatory macrophage presence and increased the extent of arteriogenesis in collateral vessels feeding the delayed flap. Conversely, loss-of-function studies that effectively reduced the macrophage population significantly attenuated the arteriogenic response. Together, these studies confirm that recruited M2 macrophages are instrumental in orchestrating vascular remodeling in fat and help elucidate the interplay between the microvasculature and immune system in remodeling adipose tissue. More broadly, these findings support the therapeutic targeting of macrophages to enhance and/or accelerate the desirable vascular adaptations invoked by the flap delay procedure. 


\section{Materials and Methods}

Bone marrow isolation for macrophage injection study

A previously published protocol ${ }^{135}$ was used to isolate bone marrow of twelve week old male $\mathrm{C} 57 \mathrm{BL} / 6$ mice. Briefly, mice were euthanized via $\mathrm{CO}_{2}$ asphyxiation and the skin of the back legs was removed using sterile iris scissors. Muscle, fat, and fascia surrounding the fibula, tibia, and femur were removed with sterile forceps and No. 11 scalpel blades. After removing the majority of muscle, fat, and fascia, the back legs were excised from the animal and stored in a sterile container with sterile PBS. Under a biosafety cabinet, the femur was dissociated from the tibia/fibula at the knee joint and complete removal of the fascia, fat, and muscle proceeded with a No. 11 scalpel blade. After the bones (fibula and femur) were void of surrounding tissue, the ends of the bones were cut with sterile iris scissor to expose the internal bone marrow compartment. A 23-gauge needle was used to pierce two holes in the bottom of sterile $200 \mu \mathrm{L}$ polymerase chain reaction (PCR) tubes. Open-ended bones (one fibula and one femur) were placed in the PCR tubes and closed and these closed PCR tubes were placed into sterile $2 \mathrm{~mL}$ microcentrifuge tubes (two total tubes per mouse). The tubes were spun at $3000 \mathrm{RPM}$ in a microcentrifuge for 10 seconds to extract the bone marrow compartment via centrifugal force. Successful extraction was confirmed by observation of a dark red pellet at the bottom of the $2 \mathrm{~mL}$ microcentrifuge tube and bones that were no longer red in color. Repeated centrifugation was necessary in some trials to entirely extract bone marrow. The bone marrow pellet was resuspended in $1 \mathrm{~mL}$ of erythrocyte lysis buffer (eBioscience) and incubated at room temperature for 8 minutes. After incubation, 
samples from one mouse were combined into a $15 \mathrm{~mL}$ centrifuge tube and spun at $1100 \mathrm{RPMs}$ for 5 minutes. The supernatant was discarded and the pellet from one mouse was suspended in $50 \mathrm{mLs}$ of media (RPMI media with $1 \%$ penicillin/streptomycin, $20 \mathrm{mM} \mathrm{HEPES,} \mathrm{10 \%} \mathrm{fetal} \mathrm{bovine} \mathrm{serum,} \mathrm{and} \mathrm{10 \%} \mathrm{L929} \mathrm{cell}$ conditioned media). $10 \mathrm{mLs}$ of cell suspension were added to each $100 \mathrm{~mm}$ cell culture dish (total of 5 dishes per mouse) and were placed in an incubator that maintained $37^{\circ} \mathrm{C}$ and $5 \% \mathrm{CO}_{2}$.

\section{Culture of bone marrow derived macrophages}

Cells were checked daily for contamination and the media was changed every four days until use. The bone marrow cells will be fully differentiated into macrophages after seven days in culture. After seven days of differentiation in the L929 conditioned media, the media was switched to RPMI media without L929 conditioned media (1\% penicillin/streptomycin, $20 \mathrm{mM}$ HEPES, 10\% fetal bovine serum). Cells were maintained in culture in this RPMI media for no more than two weeks and were not passaged.

\section{Differentiation of macrophages into M2 phenotype}

Media was supplemented with interleukin 4 (IL-4) to polarize bone marrow derived macrophages into the M2 phenotype. $10 \mu \mathrm{L}$ of $10 \mu \mathrm{g} / \mathrm{mL}$ IL- 4 was added to $10 \mathrm{mLs}$ of fresh RPMI media (without L929 conditioned media) for a final concentration of IL- 
4 of $10 \mathrm{ng} / \mathrm{mL}$. Spent media was removed and the IL-4 containing media was added and incubated for 24 hours prior to use of the cells.

\section{Vybrant DiI labeling of M2 macrophages prior to injection}

After polarization with IL-4 supplemented media, the cells were labeled with Vybrant DiI cell-labeling solution (ThermoFisher Scientific) in order to visualize the cells post-injection into the inguinal fat pad. Cells were removed from dishes with a 15 minute incubation Accutase (Innovative Cell Technologies) and resuspended in RPMI media (without L929 conditioned media) at a concentration of one million cells per milliliter. $5 \mu \mathrm{L}$ of Vybrant DiI cell-labeling solution per milliliter of cell suspension was added directly to the suspension and mixed well via gentle pipetting. The cells were incubated on ice for 15 minutes protected from light. Cells were centrifuged at 1100 RPM for five minutes and washed with sterile PBS. This process was repeated three times for a total of three PBS washes and centrifugations. After the final centrifugation, the supernatant was discarded and cells were suspended at a final concentration of 300,000 cells $/ \mathrm{mL}$.

\section{Injection of Vybrant DiI labeled and unlabeled cells into inguinal fat pad}

Wild type C57BL/6 mice were anesthetized and a one-centimeter incision was made over the left and right epigastric artery. The collateral vessel entering the inguinal fat pad was found on both sides by undermining fascia and surrounding adipose tissue (Figure 2.1). Using a 27G syringe (BD Soloshot ${ }^{\mathrm{TM}} 301793$ ), $100 \mu \mathrm{L}$ of the DiI- 
labeled cell solution $(300,000$ cells $/ \mathrm{mL})$ was injected directly into the inguinal fat pad. $100 \mu \mathrm{L}$ of unlabeled cells (no Vybrant DiI labeling, 300,000 cells/mL) were injected directly in the right inguinal fat pad. Incisions were sutured closed with 8-0 nylon suture.
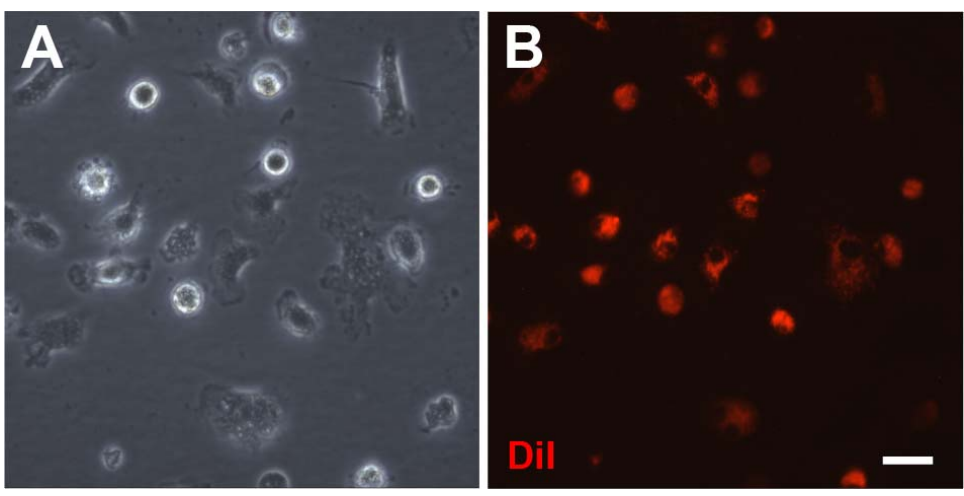

Figure 3.1: Fluorescent labeling of bone marrow derived macrophages

In vitro culturing of bone marrow derived macrophages can be fluorescently labeled with DiI. A.) Bone marrow derived macrophages display prototypical "fried egg" morphology in culture. B.) DiI, a membrane dye, labels macrophages prior to injection and is observable in same field of view. Scale bar $=50 \mu \mathrm{m}$.

Harvest and immunostaining of adipose tissue following direction cell injection

Mice were euthanized via $\mathrm{CO}_{2}$ asphyxiation three days post-injection to assess the retention of injected Vybrant DiI labeled cells and the total number of macrophages present post injection. The area of the inguinal fat pad surrounding the collateral vessel was harvested as described and depicted in Figure 2.1. Samples of the tissue injected with Vybrant DiI labeled cells were divided into three, small $\left(\sim 1 \mathrm{~mm}^{3}\right)$ pieces for immunostaining. All three samples were submerged in $50 \mu \mathrm{L}$ of $4 \%$ paraformaldehyde solution and incubated overnight at $4^{\circ} \mathrm{C}$ protected from light. All 
samples were washed with PBS three times. Sample 1 was mounted on a gelatincoated slide and imaged immediately after PBS washing as described previously in Chapter 2. Samples 2 and 3 were placed in $100 \mu \mathrm{L}$ of $5 \%$ mouse serum in $0.3 \%$ (volume/volume) Triton X-100/PBS for 3 hours at room temperature to permeabilize and block the tissue. The permeabilization and blocking solution was removed. Sample 2 was submerged in $100 \mu \mathrm{L}$ of Alexa Fluor 488 conjugated isolectin (1:300) and Alexa Fluor 647 anti-mouse CD68 (1:200) diluted in 5\% mouse serum in 0.3\% Triton X-100/PBS. Sample 3 was submerged in $100 \mu \mathrm{L}$ of Alexa Fluor 488 conjugated isolectin (1:300) and Alexa Fluor 647 anti-mouse CD206 (1:200) diluted in $5 \%$ mouse serum in $0.3 \%$ Triton X-100/PBS. Samples 2 and 3 were incubated overnight on a rocker at $4^{\circ} \mathrm{C}$ protected from light. Samples were washed five times for five minutes each wash with $0.3 \%$ Triton X-100/PBS and then mounted on gelatin-coated slides as described previously in Chapter 2. All samples were imaged using confocal microscopy as described previously in Chapter 2.

\section{Injection of Vybrant DiI labeled cells during ligation surgery}

In a separate study, ligation surgeries were performed on the left and right side of C57BL/6 mice as described previously in Chapter 2. Immediately after severing the epigastric artery on the left side, 30,000 Vybrant DiI labeled M2 polarized cells (100 $\mu \mathrm{L}$, concentration of 300,000 cells $/ \mathrm{mL}$ ) were injected directly into the inguinal fat pad surrounding the area where the collateral vessel enters. To serve as a vehicle control for the same mouse, $100 \mu \mathrm{L}$ of sterile PBS was injected directly into the right 
inguinal fat pad where the collateral vessel enters immediately after severing the epigastric artery. Incisions on both sides were closed with 8-0 nylon suture.

Harvest and immunostaining of collateral vessel and surrounding adipose tissue after ligation

Collateral vessel and surrounding adipose tissue was harvested three days post surgery and stained for $\alpha$-smooth muscle FITC (1:200), and Alexa Fluor 647 antimouse CD206 (1:200) as described previously in Chapter 2. Vessel diameters were quantified for the feeding vessel, the first branch, and the second branch, and $\mathrm{CD}^{206^{+}}$cells were quantified.

\section{Fabrication of FTY720 loaded and unloaded PLAGA films}

A previously published solvent-casting technique was used to fabricate PLAGA thin films loaded with FTY720138. Briefly, $350 \mathrm{mg}$ of PLAGA (Sigma, 50:50 lactide:glycolide, $0.59 \mathrm{dL} / \mathrm{g}$ inherent viscosity) was mixed with $2 \mathrm{~mL}$ of dichloromethane in a glass scintillation vial until the polymer was completely dissolved. For drug loaded thin films, $1.75 \mathrm{mg}$ of FTY720 (Cayman Chemical) was mixed into the polymer/solvent solution prior to casting. For unloaded thin films, this step was omitted. The polymer/solvent/drug solution was quickly poured into a Bytac Teflon lined P35 glass Petri dish. The Petri dish was sealed with Parafilm and was stored and allowed to dry at $-20^{\circ} \mathrm{C}$ for 7 days. After drying, films were stored at room temperature in a desiccator until ready to use. 
Film preparation for in vivo use

Prior to implantation into animal model, the PLAGA thin films were sterilized. 1 $\mathrm{mm}$ diameter thin films were extracted using a $1 \mathrm{~mm}$ diameter biopsy punch (Milltex). Films were soaked in 70\% ethanol for thirty seconds followed by a soak in sterile PBS for 30 seconds.

\section{Inguinal ligation and sham surgeries and thin film placement}

Ligation surgeries were performed on left and right side of $\mathrm{C} 57 \mathrm{BL} / 6$ mice as described previously in Chapter 2. Immediately after severing the epigastric artery, a $1 \mathrm{~mm}$ diameter, FTY720-loaded or unloaded PLAGA thin film was placed immediately adjacent to the collateral vessel entering the inguinal fat pad. The thin film was placed underneath the fascial layer so as to minimize displacement away from the inguinal fat pad and region of interest (Figure 3.4A,B). The left side of the mouse had the FTY720 loaded PLAGA film while the right side had an unloaded PLAGA film. Sham surgeries were performed on separate mice on both the left and right side of $\mathrm{C} 57 \mathrm{BL} / 6$ mice. Again, the left side had the drug loaded thin film and the right side had the unloaded thin polymer film. Incisions were closed with 8-0 nylon suture (Ethilon). The process described above was also repeated with sham surgeries on the left and right side in a separate set of experiments. 
Harvest and immunostaining of collateral vessel and surrounding adipose tissue after ligation

Collateral vessel and surrounding adipose tissue around the PLAGA thin film was harvested three days post surgery and stained for Alexa Fluor 488 anti-mouse CD206 (1:200), $\alpha$-smooth muscle Cy3 (1:200), and Alexa Fluor 647 anti-mouse CD68 (1:200) as described previously in Chapter 2. Vessel diameters were quantified for the feeding vessel, the first branch, and the second branch, and CD206 $6^{+}$cells were quantified.

\section{Injection of clodronate and control liposomes into inguinal fat pad}

To assess the effectiveness of clodronate liposome-mediated depletion of macrophages within the inguinal fat pad, we performed direct injection of clodronate loaded liposomes and control liposomes (PBS loaded liposomes) into the inguinal fat pad. A $1 \mathrm{~cm}$ incision was made above the epigastric artery and blunt dissection was used to locate the collateral vessel entering the side of the inguinal fat pad (Figure 2.1). Using a 27G syringe (BD Soloshot ${ }^{\mathrm{TM}} 301793$ ), $100 \mu \mathrm{L}$ of clodronate-loaded liposomes and control liposomes were injected directly into the inguinal fat pad at the insertion site of the collateral vessel. Successful injection was confirmed by visual observation of a bubble formed within the inguinal fat pad. Clodronate liposomes were injected into the right inguinal fat pad of the mouse and control liposomes were injected into the left inguinal fat pad. After injection the incision was closed with 8-0 nylon suture (Ethilon). 
Immunostaining of adipose tissue and macrophage quantification following liposome injection

To determine the temporal effect of clodronate liposome injection on macrophage depletion, adipose tissue surrounding the collateral vessel was harvested as described previously in Chapter 2 at 6 hours, 12 hours, and 24 hours. Harvested adipose tissue was stained with Alexa Fluor 568 conjugated isolectin GS-IB from $_{4}$ Griffonia simplicifoli (1:300) and Alexa Fluor 647 anti-mouse CD68 (1:200) as described previously in Chapter 2. Three unique fields of view were acquired for each sample as $40 \mu \mathrm{m}$ z-stacks and the number of CD68 ${ }^{+}$cells were quantified using ImageJ to assess macrophage depletion.

\section{Ligation of epigastric artery coupled with liposome injections}

After discerning the temporal effect of clodronate liposomes, we performed a separate set of experiments. To evaluate the effect of macrophage depletion on the vascular remodeling observed within the inguinal fat pad (Chapter 2), we injected liposomes directly into the inguinal fat pad during surgery as described in Chapter 2. Briefly, following ligation of the epigastric artery in C57BL/6 mice, $100 \mu \mathrm{L}$ of clodronate loaded liposomes (left side of mouse) or control liposomes (right side of mouse) were injected directly into the inguinal fat pad at the insertion site of the collateral vessel. Surgical incisions were closed with 8-0 nylon suture and mice were allowed to recover for three days post surgery prior to tissue harvest. 
Harvest and immunostaining of collateral vessel and surrounding adipose tissue after ligation

As described in Chapter 2, the collateral vessel was carefully removed to retain structural integrity in order to evaluate vessel diameter. Adipose tissue surrounding the collateral vessel was also harvested during the removal process and was used for macrophage quantification. Harvested collateral vessels and surrounding adipose tissue samples were stained concurrently with $\alpha$-smooth muscle Cy3 (1:200) and Alexa Fluor 647 anti-mouse CD68 (1:200) as described previously in Chapter 2. Four unique fields of view were acquired for each sample as $40 \mu \mathrm{m}$ z-stacks and the number of $\mathrm{CD} 68^{+}$cells were quantified using ImageJ. The diameter of the feeding vessels, the first branch, and the second branch were quantified in ImageJ.

\section{Statistical analysis}

Statistical tests performed are indicated in each figure caption. Statistical significance was asserted at $\mathrm{p}$-values $<0.05$. All data are presented as average + standard deviation. 


\section{Results}

DiI labeling allows for visualization of M2 differentiated macrophages

Murine bone marrow was harvested, and bone marrow-derived macrophages were cultured for 7 days in macrophage differentiation media as described above. After polarization to M2 phenotype by IL-4 supplementation, the in vitro cells were stained with DiI and fluorescence microscopy was used to visually confirm successful staining. The in vitro M2 macrophages displayed the expected "fried egg" appearance as is typical in culture (Figure 3.1A). Labeling the cells with DiI allowed for visualization of the cell membrane via florescence microscopy (Figure 3.1B).

\section{DiI labeled cells remain in inguinal fat pad three days post-injection}

To assess the retention capability of the directly injected DiI-labeled macrophages, we injected 30,000 labeled cells into the inguinal fat pad at the site where the collateral vessel enters. On the contralateral side, we injected unlabeled macrophages to serve as a fluorescence control. We wanted to determine if the injected cells were retained in the tissue for three days post-injection, which is the time frame in which we observed the remodeling response in Chapter 2. Tissue was excised three days post-injection for DiI-labeled and unlabeled cells. After fixation with $4 \%$ PFA and without permeabilizing the tissue (Sample 1 above), we mounted the tissue and visualized a substantial number of retained $\mathrm{DiI}^{+}$cells in the region of interest (Figure 3.2B). We did not observe any fluorescence signal in the tissues that were injected with unlabeled cells (Figure 3.2A), which indicates that the red signal observed in Figure 3.2B is that of the injected cells. 

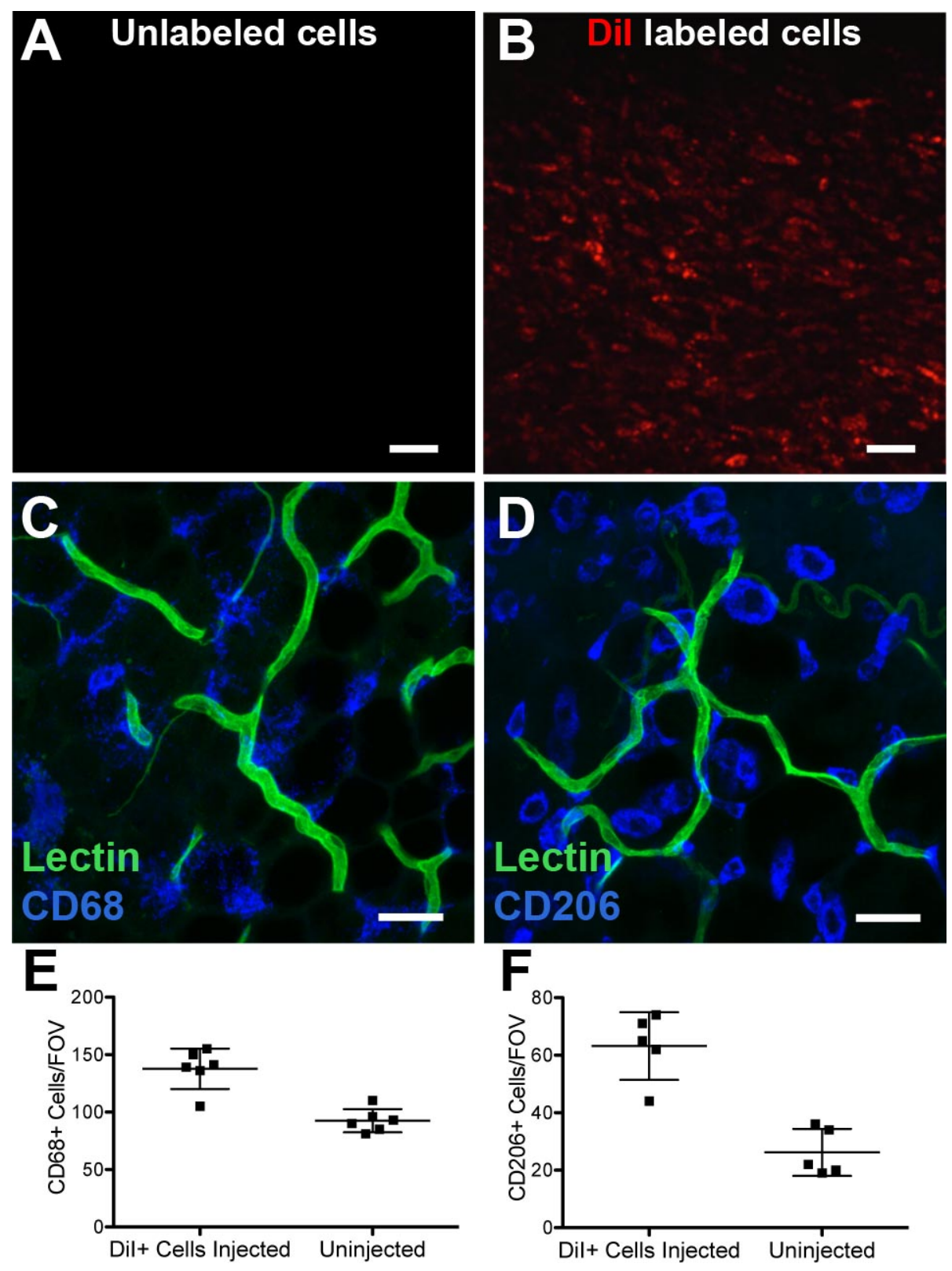

Figure 3.2: In vivo injection of bone marrow-derived macrophages Fluorescent labeling reveals an increase in $\mathrm{CD}^{2} 8^{+}$and $\mathrm{CD}^{206^{+}}$cells in injected inguinal fat pads. A.) No fluorescent signal observed in inguinal fat pads injected with unlabeled cells. Scale bar $=50 \mu \mathrm{m}$. B.) Significant presence of DiI labeled cells in injected inguinal fat pads. Scale bar $=50 \mu \mathrm{m}$. C.) Fluorescent labeling of inguinal fat pad reveals increased $\mathrm{CD} 8^{+}$cell presence in injected inguinal fat pads. Scale bar $=20 \mu \mathrm{m}$. D.) Fluorescent labeling of inguinal fat pad reveals increased $\mathrm{CD}^{206^{+}}$cell presence in injected inguinal fat pads. Scale bar $=20 \mu \mathrm{m}$. E.) Increased number of $\mathrm{CD} 68^{+}$cells in injected inguinal fat pads when compared to uninjected inguinal fat pads. $n=3$ mice. F.) Increased number of $\mathrm{CD}^{206^{+}}$cells in injected inguinal fat pads when compared to uninjected inguinal fat pads. $n=3$ mice. Data are mean \pm standard deviation. 
DiI-labeling in injected macrophage is lost after tissue processing

We reserved pieces of adipose tissue from cell-injected inguinal fat pads to determine if the cells injected could be co-labeled with macrophage markers as stated above in the materials and methods section (Samples 2 and 3). This colabeling of injected cells with macrophage markers (CD68 and CD206) would allow us to visualize if the injected DiI-labeled cells were still expressing macrophage phenotypes in vivo. Prior to staining with macrophage antibodies, a permeabilization step was required to facilitate antibody binding with the adipose tissue. We did not observe DiI signal (red channel) in either of the samples, but detected robust CD68, CD206, and lectin staining in these tissues (Figure 3.2C,D). The lack of DiI signal is likely due to the permeabilization step and the ensuing egress of the DiI dye from the cellular membrane ${ }^{206}$. We believe that the injected cells are still present in Samples 2 and 3 based on our findings in Figure 3.2B in which we observed many $\mathrm{DiI}^{+}$cells within the tissue.

Increase in $\mathrm{CD}^{6} 8^{+}$and $\mathrm{CD}^{206^{+}}$cells in cell injected tissue without ligation surgery We quantified the number of $\mathrm{CD} 68^{+}$and $\mathrm{CD} 206^{+}$cells in cell injected and uninjected tissue to determine if injection of in vitro differentiated macrophages increased the total number of macrophages three days post-injection. We performed these experiments prior to injecting cells during ligation surgeries to ensure that the $\mathrm{CD}^{+} 8^{+}$and $\mathrm{CD} 206^{+}$cells were increased in injected tissues. We observed an increase in both $\mathrm{CD} 68^{+}$cells and CD206 ${ }^{+}$cells in injected inguinal fat pads when compared 
to uninjected inguinal fat pads (Figure $3.2 \mathrm{E}, \mathrm{F}$ ). While we were not able to directly correlate the injected DiI labeled cells with this observed increase due to the lack of DiI signal as discussed above, we believe that this increase in $\mathrm{CD}_{6} 8^{+}$and $\mathrm{CD} 206^{+}$ cells is due to our injection of in vitro macrophages.

Direct injection of M2 macrophages increases $C D 206^{+}$cell counts within inguinal fat pad after ligation surgery

During ligation surgeries, 30,000 M2 differentiated macrophages (100 $\mu \mathrm{L})$ were injected into the left inguinal fat pad, while $100 \mu \mathrm{L}$ of sterile PBS was injected into the right inguinal fat pad. Three days after ligation surgery, we observed a significant increase in the number of $\mathrm{CD} 206^{+}$cells in the tissue injected with M2 differentiated cells when compared to PBS injected fat pads (p-value < 0.05) (Figure 3.3A). Within cell injected fat pads we found an average of $87 \pm 23$ (S.D.) CD206 ${ }^{+}$ cells, while in PBS injected fat pads we found an average of $51 \pm 10$ (S.D.) CD206 ${ }^{+}$ cells (Figure 3.3A). Interestingly, the $\mathrm{CD}^{206^{+}}$cell counts within PBS injected fat pads were in close agreement with $\mathrm{CD} 206^{+}$cell counts observed in ligation surgeries performed in Chapter 2 (Figure 2.7C). Taken together with our previous retention studies (above), these CD206 ${ }^{+}$cell count data suggest that injected M2 macrophages reside within the fat pad three days post-surgery. 
Direct injection of M2 macrophages increases collateral vessel diameters after ligation surgery

We quantified collateral vessel diameters and observed a significant increase upon pairing each vessel branch with its corresponding branch in the contralateral fat pad, whose feeding artery also underwent surgical ligation but received vehicle control (PBS) injection instead of cells (Figure 3.3B). We also compared these data to original data in Chapter 2 and observed similar diameters for PBS injected tissues and tissues that had only been ligated (Figure 3.3B). Interestingly, it appears that direct injection of M2 macrophages leads to an enhanced enlargement of the collateral vessel following ligation surgery. Based on these data, it seems plausible that direct supplementation of M2 differentiated macrophages enhances the arteriogenic response of the collateral vessel observable by collateral vessel enlargement.

FTY720-loaded and unloaded films remain in place post-implantation Efficient drug delivery is crucial for these studies and therefore optimization of placement of the film was necessary in order for the film to not shift after placement. A thin fascial layer is present above the mouse inguinal fat pad and this was used to hold the film in place as described in the methods. After three days post-implantation the film was located within a few micrometers of the region where it was implanted (Figure 3.4A,B) in all mice used in the studies. Notably, the FTY720 and unloaded PLAGA films were opaque and white in color upon harvest at 
day three. This finding is consistent with previous work in our lab and it is unknown the cause of this color change.
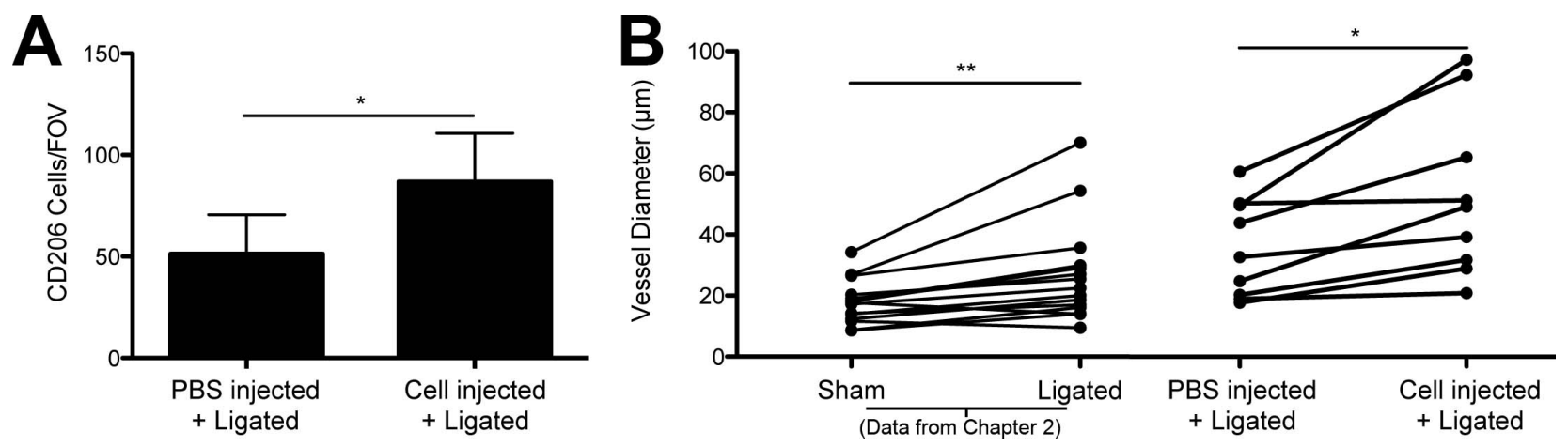

Figure 3.3: Injection of bone marrow-derived macrophages into remodeling inguinal fat pad

Injection of bone marrow-derived macrophages increased the number of CD206 ${ }^{+}$cells and increased the diameter of the collateral vessel when compared to PBS injected inguinal fat pads. A.) Quantification of the number of CD206 $6^{+}$cells/FOV. Student's t-test, ${ }^{*}=p$-value $<0.05$. Data are mean + standard deviation. B.) Individual diameter measurements for PBS injected tissues were paired with corresponding branch diameter measurements for cell injected tissues (in the same mouse). Cell injected collateral vessels have increased diameters when compared to corresponding $P B S$ injected collateral vessels. Sham and ligated data from Chapter 2 for presented for comparison purposes. Paired t-test, ${ }^{* *}=p$-value $<0.01,{ }^{*}=p$-value $<0.05 . n=3$ mice for all experiments, 4 unique FOVs for CD206 $6^{+}$cell quantification.

FTY720-loaded films increase $\mathrm{CD}_{206^{+}}$cell recruitment to ligated tissue

FTY720-loaded films significantly increased the number of CD206 ${ }^{+}$cells in the ligated tissue compared to unloaded film three days after ligation surgery ( $\mathrm{p}$-value $<$ 0.001). FTY720-loaded films had an average of $93 \pm 25$ (S.D.) CD206 ${ }^{+}$cells/FOV while unloaded films had an average of $51 \pm 14$ (S.D.) $\mathrm{CD}_{206^{+}}$cells/FOV (Figure 3.4C). In comparison to our original ligation studies in Chapter 2 (Figure 2.7C), the unloaded films had approximately the same number of $\mathrm{CD}^{206^{+}}$cells/FOV suggesting that the unloaded film does not induce significant changes in the 

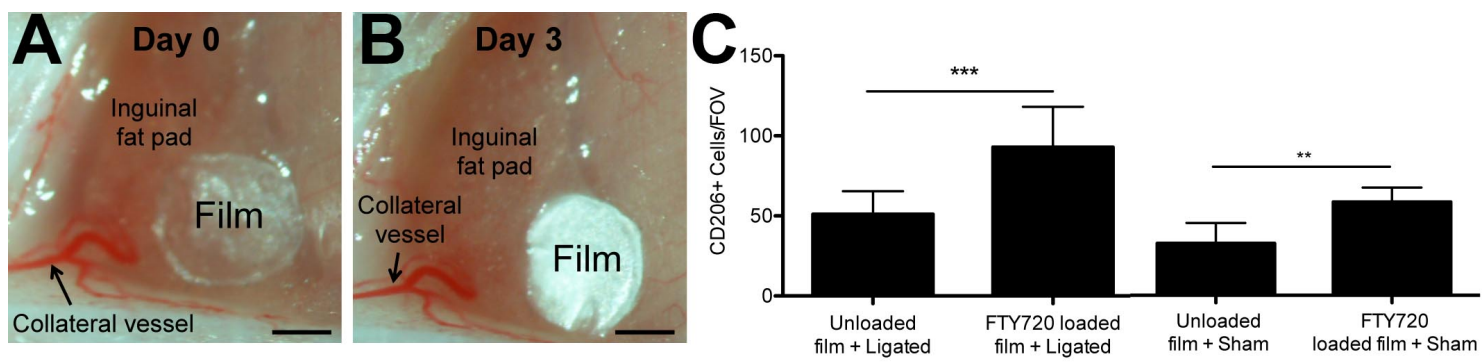

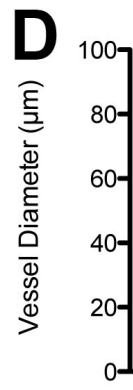

$* *$
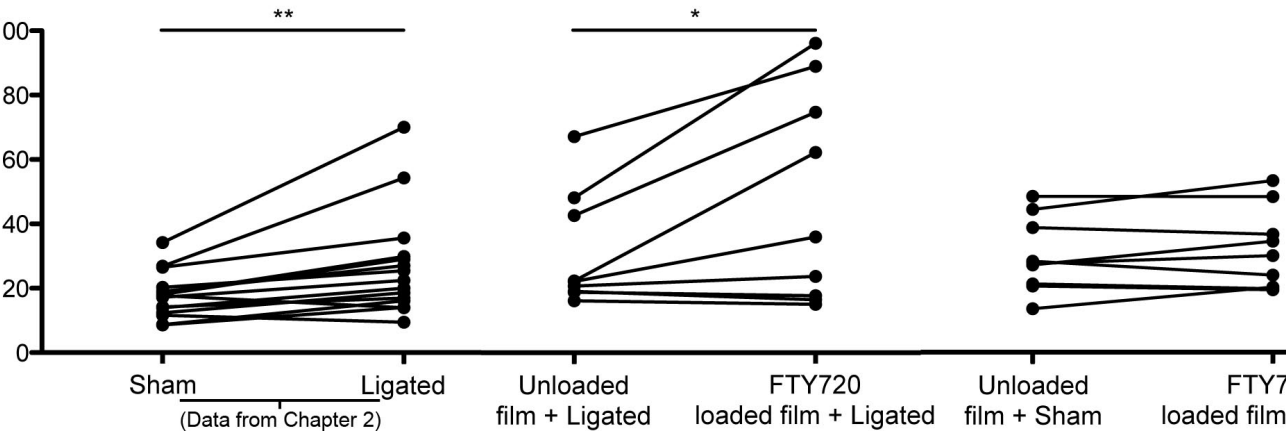

Figure 3.4: FTY720 application to remodeling inguinal fat pad

FTY720-loaded and unloaded PLAGA films were applied to remodeling fat pads and enhanced arteriogenic response was observed by FTY720-loaded films. A.) Representative image of PLAGA film placement during surgery. Scale bar $=500 \mu \mathrm{m}$. B.) Representative image of PLAGA film three days postsurgery in near exact location as Panel A. Scale bar $=500 \mu \mathrm{m}$. C.) Quantification of $\mathrm{CD}^{206^{+}}$cell counts for unloaded and FTY720-loaded PLAGA films three days after sham and ligation surgery. Quantification reveals a significant increase in the number of $C D 206^{+}$cells in ligated tissue for FTY720-loaded films when compared to unloaded films. After sham surgery a significant increase in the number of $\mathrm{CD}^{206^{+}}$cells is observed in FTY720 treated tissue. Student's t-test, ${ }^{*}=p$-value $<0.05$. D.) Individual diameter measurements for collateral vessel of FTY720-loaded film fat pads were paired with corresponding branch diameter measurements for unloaded film (in the same mouse). A significant increase in the diameter of the collateral vessels were observed for FTY720-loaded film fat pads when compared to the unloaded film fat pads. No significant increase was seen in the sham surgery trials between FTY720-loaded and unloaded films. Sham and ligated data from Chapter 2 for presented for comparison purposes. Paired t-test, ${ }^{*}=$ p-value $<0.05 . n=3$ mice for all experiments, 4 unique FOVs for $\mathrm{CD}^{206^{+}}$cell quantification.

remodeling response. Interestingly, the FTY720-loaded films had a much greater number of $\mathrm{CD}^{206^{+}}$cells/FOV compared to our original studies (93 vs. 45 CD206 ${ }^{+}$ cells/FOV). Taken together, these data suggest that FTY720 aids in the recruitment 
of anti-inflammatory macrophages to the remodeling fat pad, similar to what has been observed in skeletal muscle ${ }^{138}$.

\section{FTY720-loaded films enhance collateral vessel enlargement in ligated tissue}

Pairing collateral vessels of inguinal fat pads treated with unloaded versus FTY720loaded films reveals that FTY720-loaded films significantly increased collateral vessel diameters following ligation surgeries (Figure 3.4D). Data from Chapter 2 revealed an increase in the diameter of collateral vessel of ligated fat pads when compared to sham fat pads and was indicative of an arteriogenic response (Figure 2.4B). Importantly, the vessel diameters of the collateral arterioles in the ligated tissue treated with unloaded film are not significantly different than those that were ligated but did not receive any film, suggesting that the PLAGA by itself does not have a significant effect on the collateral vessel remodeling process (Figure 3.2D). These present studies suggest that FTY720 amplifies the observed increase in collateral vessel diameters following ligation and that coupling the ligation surgery with FTY720 delivery may induce more profound vascular remodeling within adipose tissue.

FTY720 does not affect vessel diameter following sham surgery

FTY720-loaded and unloaded films were applied following sham surgeries. There was no significant difference in collateral vessel diameters for FTY720-loaded or unloaded films following the sham procedure (Figure 3.4D). Interestingly, there was 
a statistically significant increase in the number of CD206 ${ }^{+}$cells in FTY720-loaded tissue (average $59 \pm 9$ (S.D.) CD206 ${ }^{+}$cells/FOV) when compared to unloaded film tissue $\left(33 \pm 13\right.$ (S.D.) CD206 ${ }^{+}$cells/FOV) (p-value < 0.05) (Figure 3.4C). These data suggest that while FTY720 recruits CD206 ${ }^{+}$cells to the tissue in the absence of ligation, collateral vessel enlargement is contingent on alteration of blood flow in the main feeding artery. Further, these data suggest that, at least in the absence of ligation-induced blood flow alterations, FTY720 does not directly affect the proliferation and activation of vascular smooth muscle cells or endothelial cells in the collateral vessel.
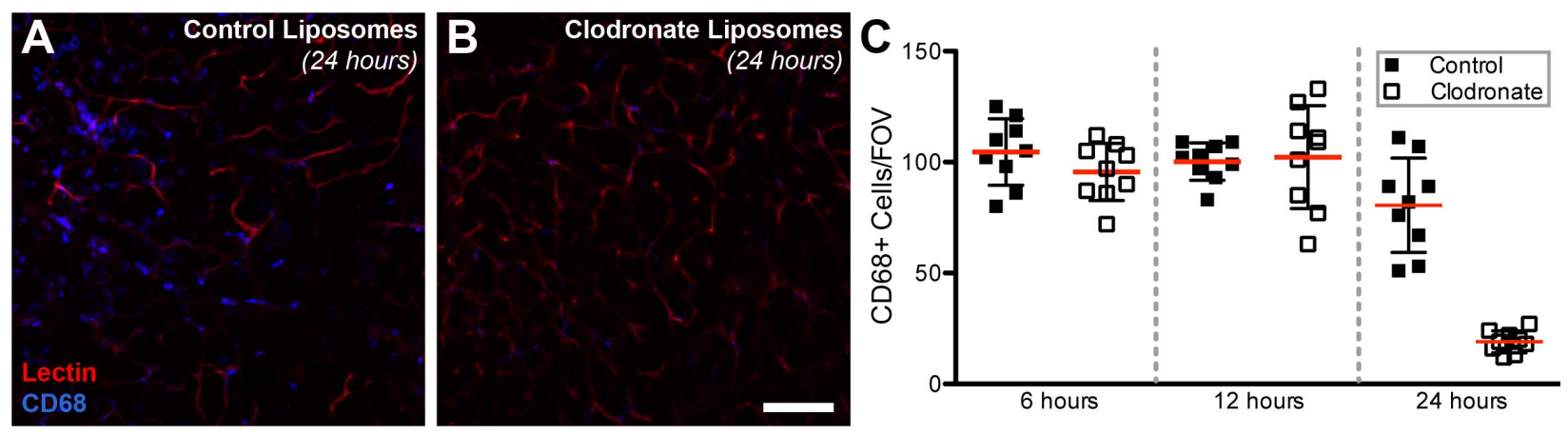

Figure 3.5: Clodronate depletion of macrophages within inguinal fat pad Direct injection of clodronate liposomes into inguinal fat pad are able to significantly deplete local macrophage populations (CD68 cells) after 24 hours. A.) Representative image of control liposome injected inguinal fat pad at 24 hours shows expected $\mathrm{CD} \mathrm{8}^{+}$cell presence. B.) Representative image of clodronate liposome injected inguinal fat pad at 24 hours shows significantly fewer CD68 $8^{+}$cells. C.) Quantification of $\mathrm{CD}^{2} 8^{+}$cells at 6 hours, 12 hours, and 24 hours. A significant decrease in the number of $\mathrm{CD}^{+} 8^{+}$cells was observed 24 hours post-injection in clodronate liposome injected inguinal fat pads. Control liposome injected fat pads did not have depleted CD68 cells. Scale bar $=100 \mu \mathrm{m}, n=3$ mice for each time point, 3 unique FOVs for $\mathrm{CD} 68^{+}$cell quantification.

Clodronate liposomes deplete local macrophage populations 24 hours after injection

Following injection of clodronate-loaded liposomes or control liposomes we quantified the number of $\mathrm{CD} 68^{+}$cells within the region of interest in the inguinal 
fat pad. Twenty-four hours after injection of clodronate liposomes, we observed a reduced number of $\mathrm{CD} 68^{+}$cells in the tissue relative to tissue injected with control liposomes (Figure 3.5A,B). The number of $\mathrm{CD}^{+} 8^{+}$cells/FOV was significantly reduced to an average of $19 \pm 5$ (S.D.) $\mathrm{CD}^{+} 8^{+}$cells/FOV when compared to control liposomes average of $80 \pm 19$ (S.D.) $\mathrm{CD}^{+} 8^{+}$cells/FOV (Figure 3.5C). After six and twelve hours, there was no a significant difference in the number of $\mathrm{CD}^{+} 8^{+}$cells between control and clodronate liposome treated inguinal fat pads. Taken together, these data suggest that clodronate liposome injection significantly reduces macrophage population size in the adipose tissue and that control liposomes do not significantly affect adipose macrophage counts.
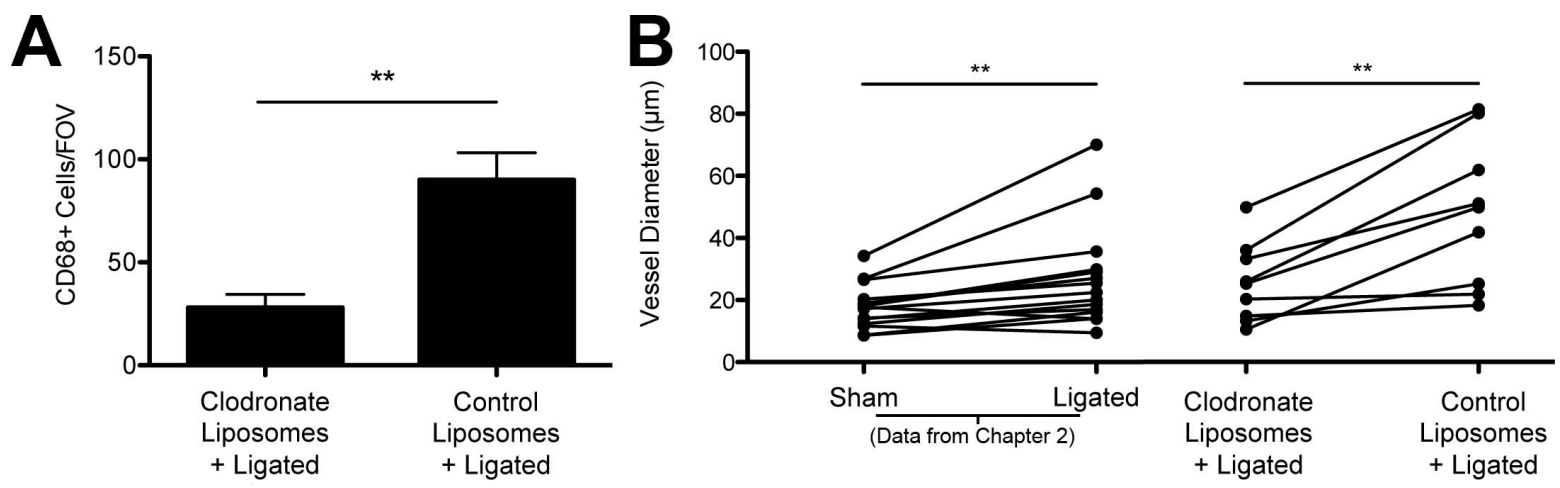

Figure 3.6: Injection of clodronate liposomes within inguinal fat pad Clodronate liposomes depleted the local macrophage population after ligation surgery altering microvascular remodeling response. A.) Quantification of $C D 68^{+}$cells reveal a significant decrease in the number of $C D 68^{+}$cells within clodronate liposome treated tissue compared to control liposome treated tissue. Student's t-test, ${ }^{* *}=p$-value $<0.01 \mathrm{~B}$.) Individual diameter measurements for clodronate liposome injected tissues were paired with corresponding branch diameter measurements for control liposome injected tissues (in the same mouse). Control liposome injected collateral vessels have increased diameters when compared to corresponding clodronate liposome injected collateral vessels. Sham and ligated data from Chapter 2 for presented for comparison purposes. Paired $t$-test, ${ }^{* *}=p$-value $<0.01 . n=3$ mice for all experiments, 4 unique FOVs for $\mathrm{CD} 68^{+}$cell quantification. 
Injection of clodronate liposomes reduces $C D 68^{+}$cell counts after ligation surgery

Tissue treated with either control or clodronate-loaded liposomes were harvested three days after the ligation surgery was performed. Fat pads that had been injected with clodronate liposomes experienced a significant reduction in the number of $\mathrm{CD}^{2} 8^{+}$cells (average $28 \pm 7$ (S.D.) $\mathrm{CD} 68^{+}$cells/FOV) when compared to control liposomes injected fat pads (average $90 \pm 13$ (S.D.) $\mathrm{CD}^{+}$cells/FOV) (pvalue $<0.01$ ) (Figure 3.6A). These data support that clodronate liposomes effectively reduce macrophage counts in the inguinal flap delay model.

Injection of clodronate liposomes reduces collateral vessel diameters following ligation surgeries

Pairing respective collateral vessel branches between clodronate liposome-treated and control liposome-treated fat pads revealed a significant decrease in the collateral vessel diameters of clodronate liposome fat pads (Figure 3.6B). These data suggest that macrophages play an important role in the enlargement of collateral vessel diameters, and that this enlargement may be abrogated by depletion of the macrophage population within the tissue.

Further, comparison between adipose tissues that only underwent surgical ligation of the feeding artery (Chapter 2) and those that underwent clodronate liposome treatment in addition to feeding artery ligation reveals a relative decrease in the overall diameters of the collateral vessels of tissues that received clodronate liposomes (Figure 3.6B). While this comparison spans two trials that were 
conducted independently, it suggests that depletion of macrophages within the remodeling fat pad reduces the arteriogenic response. 


\section{Discussion}

The dynamic nature of the microvascular system allows for rapid adaptation of its structure and function in response to biochemical and biomechanical cues. The plastic surgery community has exploited these microvascular remodeling responses by utilizing a surgical pre-treatment technique to aid in the repair of soft tissue defects by using autologous flaps. "Flap delay" involves the alteration of blood flow into the tissue flap prior to transfer to recipient site and it is presumed that this alteration leads to better survival upon transplantation to the recipient site. In our previous studies (Chapter 2), we formulated a murine model of flap delay and observed a significant arteriogenic response (enlargement of collateral vessel) in the adipose flap in response to the ligation of the main vascular pedicle. Preceding this arteriogenic response, we observed an increase in the recruitment of circulating monocytes and local increases in the number of CD206 ${ }^{+}$cells (M2 macrophages). We also observed local proliferation of recruited macrophages surrounding the collateral vessels, as evidenced by positive Ki-67 staining. Based on these findings, we hypothesized that M2 macrophages play a significant role in the arteriogenic response observed within the flap. The aim of these current studies was to confirm this putative mechanism by performing gain-of-function (increase local M2 macrophage populations) and loss-of-function studies (depletion of local macrophage populations) in our previously published model of flap delay. We found that both direct injection of M2 macrophages within the flap and preferential recruitment of M2 macrophages to the tissue via pharmacological means increased the remodeling collateral vessel diameter and the number of M2 macrophages in the tissue. We also 
used clodronate liposomes to locally deplete macrophage populations within the remodeling fat pad and observed decreased collateral vessel enlargement in these studies.

Although this study is the first, to our knowledge, to confirm a role for macrophages in orchestrating collateral vessel remodeling in adipose tissue, the process of collateral vessel enlargement has been studied extensively in skeletal muscle. Our group, as well as others, have demonstrated the importance of M2 macrophages in the arteriogenic response following an alteration in blood flow ${ }^{86,139}$ in skeletal muscle. Indeed, collateral vessel enlargement, a hallmark of arteriogenesis, has been shown in other tissues to be reliant on the recruitment of circulating monocytes followed by their extravasation into the interstitial tissue. Extravasated monocytes differentiate into macrophages and then secrete potent growth factors such as FGF-2, which stimulate smooth muscle cell and endothelial cell proliferation. This localized proliferation of the vascular cells leads to the expansion of the collateral vessel itself and leads to an increase in blood flow. While our study did not delineate between recruited and tissue-resident macrophage populations or assess local growth factor levels, it is a first step toward these logical follow-on studies.

Previous studies have directly supplemented or reintroduced cultured macrophages into various tissues in order to study the potential effects of macrophages on diseases or other pathological conditions. These studies have introduced macrophages intravenously ${ }^{186}$ and directly ${ }^{187-189}$ and have observed 
striking retention of these cells after delivery. We aimed to determine the effect of direct supplementation of M2 macrophages on the arteriogenic response of the inguinal fat pad. We successfully cultured bone marrow-derived macrophages and differentiated these macrophages into the M2 phenotype by using IL-4 media supplementation (Figure 3.1A). The differentiation step has been previously shown to induce M2 polarization in culture and a significant number of studies have used in vitro M2 differentiated macrophages ${ }^{135,189}$. In order to track the cultured macrophages post-injection and assess their retention time within the inguinal fat pad, we used DiI to stain the cellular membrane (Figure 3.1B). Three days after injection we observed a significant number of $\mathrm{DiI}^{+}$cells in the tissue implying that the injected cells remained within the inguinal fat pad (Figure 3.2B). We also observed an increase in the number of $\mathrm{CD}^{+} 8^{+}$cells and $\mathrm{CD} 206^{+}$cells in cell-injected tissue when compared to uninjected tissue, which corroborated the finding that the injected cells were retained within the tissue three days after injection (Figures 3.2C-F). Unfortunately, we did not observe dual staining of $\mathrm{DiI}^{+}$and $\mathrm{CD}^{+} 8^{+}$or $\mathrm{DiI}$ and CD206 and thus we were not able to "track" injected macrophages in subsequent studies. The staining protocol we have optimized staining adipose tissue requires a tissue detergent (Triton X-100) and we postulate that the detergent permeabilized the cellular membrane and subsequently released the DiI stain. However, based on observations of significant $\mathrm{DiI}^{+}$cell presence and increased $\mathrm{CD}^{+} 8^{+}$cells and $\mathrm{CD}^{206^{+}}$cells, we are confident that the in vitro cultured macrophages were retained in the tissue three days post-injection. 
We coupled ligation surgeries in the murine model of flap delay with injections of M2 differentiated macrophages. As expected, we observed a significant increase in the number of CD206 $6^{+}$cells within the cell injected tissue after ligation surgery confirming our findings from Figure 3.2. In tissues injected with M2 macrophages, there was a significant increase of collateral vessel diameters when paired with the corresponding collateral vessel of the PBS injected vehicle control inguinal fat pad (Figure 3.3B). When compared these data with our original findings presented in Chapter 2, direct cell injected fat pads have an even more pronounced collateral vessel enlargement following ligation surgery suggesting that the M2 macrophages have an effect on the enlargement (Figure 3.3B). Interestingly, the collateral vessel diameters of PBS injected tissue were approximately the same as the collateral vessels of ligated tissue from our original studies (Figure 3.3B).

Our observations from these direct injection studies implicate the importance of M2 macrophages on the arteriogenic response but several factors may temper these conclusions. First, it is unknown whether the injected cells maintain the M2 phenotype after injection into the inguinal fat pad and therefore it definitive that the injected, M2 macrophages are responsible for the observed arteriogenic response. Macrophages are extremely plastic and can polarize between phenotypes upon different environmental cues and therefore it is possible that the M2 macrophages injected no longer retain that phenotype ${ }^{133}$. For example, Jetten et al. injected M2 differentiated macrophages into healing skin wounds and observed a decrease in healing time despite the often reported anti-inflammatory capabilities of 
M2 macrophages ${ }^{189}$. Jetten et al., while not directly staining or quantifying macrophage populations during healing, surmised that M2 macrophages were polarizing in vivo to the M1 phenotype ${ }^{189}$. We were not able to co-label injected cells (DiI) with M2 macrophage markers (CD206) due to our staining protocol, but, in the future, it may be possible to utilize a different cell stain (i.e. PKH-26 ${ }^{207}$, calcein$\mathrm{AM}^{208}$ ) that is more resilient to permeabilization techniques. It may also be possible to use flow cytometry to confirm the increase in $\mathrm{CD}_{20} 26^{+}$cells within the tissue and may allow for the quantification of cells expressing DiI (or other dyes) and M2 macrophage markers. An interesting (and important) follow up experiment would be to inject non-polarized macrophages (M0) into the remodeling fat pads and see if there is an effect on the remodeling collateral vessels. If these proposed studies do not elicit the same effect as injected M2 macrophages, this may provide confidence that the enhancement in collateral vessel enlargement is due to the M2 macrophages that are retaining their phenotype in vivo. Second, we injected 30,000 M2 macrophages per inguinal fat pad, but believe that it would be important to determine the dose effect and titrate the number of injected cells. It is possible that we injected a supraphysiological number of M2 macrophages into the fat pad and the enhanced arteriogenic response observed would not be observed in a normal inflammatory response within the fat pad. In our previous studies we observed increased local proliferation of macrophages within remodeling fat pads around the collateral vessel ${ }^{181}$. It has been shown that local proliferation of macrophages within remodeling tissue is in part responsible for the observed arteriogenic response ${ }^{86,138}$. 
It is important to consider if these injected macrophages are proliferating within the fat pad as we observed in our previous studies.

A previous study conducted by Awojoodu et al. in collaboration with our group revealed that FTY720 preferentially recruited anti-inflammatory macrophages to remodeling skeletal muscle and increased the arteriogenic response in skeletal muscle ${ }^{138}$. After observing increases in collateral vessel diameter in M2 macrophage injected fat pads, we aimed to determine if FTY720 would elicit the same result due to the mechanism discussed by Awojoodu et al. FTY720 delivery via PLAGA films has been performed extensively and localized, sustained release of the drug is possible for several weeks post-implantation ${ }^{209}$. Figures $3.4 \mathrm{~A}$ and $3.4 \mathrm{~B}$ illustrate the placement of our PLAGA films during and three days after surgery and reveal minimal shifting in the film after placement. We observed significant increases in the number of CD206 ${ }^{+}$cells in the FTY720 treated tissue for both ligated and sham surgeries when compared to fat pads with unloaded films, which is expected based on previous studies (Figure 3.4C) ${ }^{138}$. Importantly, the number of $\mathrm{CD}^{206^{+}}$cells in ligated tissue and sham tissue treated with unloaded films is nearly the same as our previous findings in which we solely performed ligation or sham surgery on the epigastric artery (Figure 2.7C) ${ }^{181}$. These data suggest that the PLAGA itself does not have an effect on the recruitment of M2 macrophages and that the drug, FTY720, is likely responsible for the increased M2 macrophage presence. 
FTY720-loaded films following ligation surgery significantly increased collateral vessel diameters when compared to inguinal fat pads treated with unloaded films (Figure 3.4D). In comparison with studies in Chapter 2, the collateral vessel diameters exhibit an even greater increase in diameter in response to FTY720 presumably due to the significant increase in M2 macrophage recruitment. Following sham surgeries, the collateral vessel diameters were not significantly different between FTY720 and unloaded film treated fat pads suggesting that collateral vessels may not remodel/enlarge without an alteration in blood flow. Interestingly, though, there was an increase in CD206 ${ }^{+}$cells in these tissues suggesting that FTY720 may act on tissue-resident macrophages and polarize them into the M2 phenotype rather than relying on recruited monocytes/macrophages. The enlargement of collateral vessels after ligation surgery is expected based on previous studies ${ }^{138}$ and aligns with our hypothesis that M2 macrophages are implicated in the arteriogenic response.

While we did not quantify local FTY720 delivery or release into the surrounding tissue, we are confident that the drug was delivered based on previous release studies citing release for several weeks and the long half life (7 days) of FTY720209,210. In the future, it is important to consider the dose of FTY720 delivered as we only considered one concentration of drug within the PLAGA films. It is also possible that FTY720 is acting on other cell types within the adipose tissue and recruiting cells to aid in the arteriogenic response observed. It is well known that FTY720, or S1P, acts on a number of various cell types (mast cells ${ }^{211}$, 
neutrophils ${ }^{212,213}$, mural cells ${ }^{209}$ ), a number of which have been implicated in arteriogenesis.

In our loss-of-function study, we aimed to determine the effect of macrophage depletion on the arteriogenic remodeling response. We were able to successfully deplete the local macrophage population within inguinal fat pads 24 after injection of clodronate liposomes (Figure 3.5). Importantly, injection of control liposomes had no effect on the total number of macrophages $\left(\mathrm{CD}^{+} 8^{+}\right.$cells) within the inguinal fat pad after 6, 12, or 24 hours (Figure 3.5). Building on these findings, we coupled liposome injection (control and clodronate) with ligation of the epigastric artery to determine if the collateral vessel enlargement is abrogated following local macrophage depletion. As expected and consistent with our findings in Figure 3.5, the number of $\mathrm{CD}^{+}$cells in the clodronate liposome injected tissue was significantly decreased when compared to control liposome injected tissue (Figure 3.6A). This result was not surprising but was important to confirm efficacy of the liposome delivery during ligation surgery. Macrophage depletion had a drastic effect on the ability of the collateral vessel to enlarge as evidenced by Figure 3.6B. In fat pads that received clodronate liposomes during surgery, the collateral vessels were significantly smaller in diameter than fat pads that received control liposomes (Figure 3.6B). When compared to data collected from ligation or sham surgeries presented in Chapter 2, it appeared that diameters of collateral vessels of clodronate liposome fat pads had diameters more similar to vessel diameters of sham surgeries. Interestingly, these data suggest that the macrophage population is 
necessary for the enlargement in collateral vessel caliber following ligation surgery and that without a macrophage presence there is no enlargement. These results support our hypothesis that macrophages, specifically M2 macrophages, are in part responsible for the arteriogenic response and may be necessary in this process.

Clodronate liposomes serve as a good tool to transiently and locally deplete macrophage populations within a tissue and have been used extensively in the literature $203,205,214$. There are several considerations to take into account when considering the results of these studies. First, there are numerous phagocytic cells types other than macrophages (mast cells, neutrophils, dendritic cells) within adipose tissue that would be subjected to the phagocytic, apoptotic mechanism enacted by clodronate liposomes. While we demonstrate the ability of clodronate liposomes to deplete macrophage populations, there is also a possibility that the clodronate liposomes are depleting other cell populations within the inguinal fat pad. Importantly, some of these phagocytic cell types have been implicated in the arteriogenic response. For example, Heissig et al. showed that mast cells are necessary in the revascularization of skeletal muscle in a hindlimb ischemia model and propose that the secretion of MMP-9 and VEGF is central to this process ${ }^{100}$. The role of neutrophils in arteriogenesis is not well known but a study by Soehnlein et al. suggest that neutrophils play a role in recruitment of circulating monocytes ${ }^{99,123}$. Ohki et al. report that G-CSF administration aids in revascularization through protein secretion by neutrophils ${ }^{215}$. It is possible that phagocytic cells other than macrophages are depleted in our studies, but control 
liposomes (PBS loaded) serve as an important contralateral control that provide confidence in our findings. We injected the liposomes during the ligation surgeries and based on our previous findings expected that the macrophage population within clodronate liposome treated tissue to be significantly depleted at 24 hours (Figure 3.5). Clodronate liposome-mediated macrophage depletion is a transient effect ${ }^{201}$ and it is unclear how long the depletion effect lasts. The literature reports ranging durations of macrophage depletion ( 1 week $^{216}, 3$ weeks ${ }^{217}$ ) depending on application technique, tissue type, and volume of liposomes delivered. We are unaware of any studies in which clodronate liposomes are directly injected adipose tissue and as such cannot be certain that the macrophage depletion remains effective three days post-surgery when tissue is harvested. Further, we inject clodronate liposomes during surgery rather than 24 hours prior to surgery and thus during the first 24 hours after surgery there will still be a population of macrophages present within the tissue. In order to inject liposomes into a specific location of the inguinal fat pad (where the collateral vessel enters), we must make an incision to find the collateral vessel and cannot do this through the skin. While it would be ideal to inject clodronate liposomes into the fat 24 hours prior to injection, it is not possible without performing two surgeries, which is not permissible by our approved animal protocol. We are confident that clodronate liposome delivery during surgery is an efficient way to deplete macrophage populations for our short three-day terminal time point as evidence by the decreased $\mathrm{CD}^{+} 8^{+}$cell counts in Figure 3.6A. 


\section{Conclusions}

These studies help confirm our hypothesis and initial findings that macrophages, specifically M2 macrophages, are necessary for the collateral vessel remodeling response and arteriogenesis observed in the murine model of flap delay. Here, we demonstrate that direct addition of M2 macrophages into the inguinal fat pad and pharmacological recruitment of M2 macrophages to the inguinal fat pad increase the arteriogenic response observed. Further, we perform loss-of-function macrophage depletion studies and abolish the arteriogenic response in our murine model of flap delay. Taken together, these studies underscore the importance of macrophages in microvascular remodeling, specifically arteriogenesis, within adipose tissue. These studies may suggest a mechanism for a translatable therapeutic target that may be used to enhance the flap delay procedure.

\section{Acknowledgements}

The authors would like to thank Dr. Samantha Adamson and Dr. Norbert Leitinger for advice and assistance in harvesting and culturing the bone marrow derived macrophages. 


\section{Chapter 4}

\section{DIFFERENTIAL EFFECTS OF PROCESSING TIME AND DURATION OF COLLAGENASE DIGESTION ON HUMAN AND MURINE FAT GRAFTS}

Acknowledgements: Shruti C. Tannan, Yiqi Cao ${ }^{\mathrm{b}}$, Shayn M. Peircea,b, Kant Y. Lin ${ }^{a}$

${ }^{a}$ Department of Plastic Surgery, University of Virginia

${ }^{b}$ Department of Biomedical Engineering, University of Virginia

The text included in this chapter has been published here:

Seaman SA, Tannan SC, Cao Y, Peirce SM, Lin KY. Differential effects of processing time and duration of collagenase digestion on human and murine fat grafts. Plastic and Reconstructive Surgery 2015; 136(2): 189-199. 


\section{Abstract \\ Background}

Autologous fat graft retention is unpredictable and mechanisms of optimization are poorly understood. Attempts at improving retention utilize collagenase experimentally and clinically to isolate the stromal vascular fraction to "enhance" fat grafts. However, no standardized duration for collagenase digestion or time following fat graft harvest has been established. This study investigates the effect of 1.) time after fat graft harvest and 2.) collagenase digestion time on interstitial cell and adipocyte viability in murine fat and human lipoaspirate.

\section{Methods and results}

Murine fat and human lipoaspirate were incubated ex vivo after harvest at room temperature for 120 minutes. Additional groups were incubated with collagenase for increasing five minute intervals from 30-60 minutes. Samples from each group were stained with BODIPY to quantify intact adipocytes and LIVE/DEAD kit to quantify interstitial cell viability. With increased time post-harvest, the number of intact adipocytes in murine fat and human lipoaspirate remained unchanged. Human interstitial cells were resistant to the effect of increased time ex vivo, while murine interstitial cells decreased in viability. In both populations, increased collagenase digestion time significantly decreased the number of viable adipocytes (murine: $\mathrm{p}$-value $\leq 0.001$, human: $\mathrm{p}$-value $\leq 0.001$ ) and interstitial cells (murine: $\mathrm{p}$ value $\leq 0.001$, human: $p$-value $\leq 0.001)$ 


\section{Conclusions}

Human and murine adipocytes and human interstitial cells appear resistant to deleterious effects of increasing time following harvest. However, murine interstitial cells including are sensitive to increased time and prolonged collagenase digestion. These studies highlight the complex cellular components of fat grafts and how they respond differentially to time and collagenase digestion. 


\section{Introduction}

Clinical indications for autologous fat grafting have increased in both reconstructive and aesthetic plastic surgery in the past decade ${ }^{6,218-224}$. There has been a surge in both large-volume fat grafting utilized in breast and buttock reconstruction, as well as small-volume fat grafting in facial aesthetic and reconstructive applications, 6,8,223.

Intense interest in autologous fat derives from advantages including abundance of donor fat, ease of harvest, relative decreased cost compared to allogeneic and implant alternatives, and avoidance of foreign non-autologous implant material and related complications ${ }^{5,224}$. However, long-term volume retention of autologous fat grafts are highly unpredictable, with resorption rates ranging from $20-75 \% 5$.

Fat grafts are heterogeneous and are composed of adipocytes and interstitial cells, including endothelial cells, macrophages, pericytes, fibroblasts, adiposederived stem cells, and other blood-derived cells ${ }^{225}$. Methods for "enhancing" or supplementing the fat graft are being developed to improve graft retention rates $^{8,223,226,227}$. Adipose stem cells (ASCs) have been studied extensively for this application and other therapeutic applications. A number of recent studies have suggested that supplementation, or "enhancing" fat grafts with adipose-derived stem cells (ASCs), increases the graft retention rates ${ }^{5,8,223,226,227}$.

The most common technique for enhancing fat grafts in clinical practice and in pre-clinical murine models involves collagenase digestion of a portion of the fat graft to isolate the stromal vascular fraction (SVF) and supplementation of the fat graft with this ASC-rich solution. Collagenase dissociates the collagen-rich 
extracellular matrix (ECM) of the adipose tissue, which liberates the ASCs from the interstitial space. Although collagenase digestion is not approved for this indication in the United States, this technique is currently in clinical practice in a number of other countries, including Japan, Spain, and Korea ${ }^{8,223,226,228}$. In the laboratory setting, isolation of individual adipocytes using collagenase is commonly used in murine and human populations to study the adipogenesis cascade ${ }^{229,230}$, adipokine secretion from adipocytes ${ }^{231-233}$, adipocyte metabolism ${ }^{233}$, and immune cell recruitment to adipose tissue/adipocytes ${ }^{186,234}$.

Despite the prevalence of using collagenase digestion as a means to isolate the SVF and individual adipocytes, a standardized time for collagenase digestion of human and murine adipose tissue has not been determined. Collagenase digestion duration must be long enough to allow for adequate disaggregation of the adipose tissue and release of the interstitial cells, while also short enough to prevent deleterious cell disruption or cell death. Digestion times reported in the literature range from 30 minutes to 90 minutes, with no consensus supported by scientific evidence ${ }^{143-145}$. This study seeks to identify the optimal collagenase digestion duration that minimally disrupts viability of both adipocytes and interstitial cells.

A second important variable in both the clinical and laboratory settings is the time after adipose tissue removal from the organism and prior to transplantation (ex vivo time), which can be significant and is often overlooked as a factor affecting graft retention. For example, during large volume fat graft harvest, lipoaspirate may be ex vivo for two hours prior to reinjection. In a laboratory setting, time ex 
vivo following removal from a human or laboratory subject may also be significant prior to analysis. No studies to date have examined the effect of this time ex vivo on the viability of the adipocytes or interstitial cells in human and murine adipose tissue.

In the current study, we sought to determine: 1) how the amount of time after harvest and prior to injection transplantation (ex vivo time) affects adipocyte and interstitial cell health, and 2) if the duration of collagenase digestion affects adipocyte and interstitial cell health. We hypothesize that increasing collagenase digestion will decrease adipocyte and interstitial cell viability in both human and murine adipose tissue. Further, we hypothesize that time ex vivo will not significantly affect the viability of adipocytes and interstitial cells in human and murine adipose tissue.

A better understanding of how time after harvest and collagenase digestion affects the adipocytes and interstitial cells within adipose tissue will inform procedures for fat handling and manipulation that will improve autologous fat graft retention in the clinical setting. Further, these studies may help to standardize potential confounding variables in the laboratory research setting - the effect of time ex vivo and collagenase digestion on murine adipose tissue. 


\section{Materials and Methods}

Murine adipose tissue harvest

All procedures were performed in accordance with the University of Virginia Institutional Animal Care and Use Committee. Eight to twelve week old BALB/c mice (Charles River, Washington, MA) were humanely euthanized with $\mathrm{CO}_{2}$ asphyxiation with 3-4 mice used for each group. Immediately following asphyxiation, both inguinal fat pads were surgically excised and stored in phosphate buffered saline (PBS) until use. The time of removal ( $\mathrm{t}=0$ minutes) was noted to ensure accurate time points for subsequent studies.

\section{Human adipose tissue harvest}

Subcutaneous adipose tissue was obtained according to an approved protocol by The University of Virginia's Institutional Review Board. All human adipose tissue was acquired from intraoperative suction lipectomy from nondiabetic patients undergoing elective surgical procedures at the Department of Plastic Surgery at The University of Virginia. All six patients were female, non-diabetics and ranged in age from 25-65 with body mass indexes ranging from 22.5-27.5. Adipose tissue was incubated in a sealed container at room temperature $\left(25^{\circ} \mathrm{C}\right)$ and time of removal was noted to ensure accurate time points for subsequent studies.

Ex vivo time studies

Murine and human adipose tissues were incubated ex vivo in PBS at room temperature $\left(25^{\circ} \mathrm{C}\right)$ for time points up to 120 minutes. After incubation was 
complete, the tissue samples were divided evenly into two groups for each time point: one for BODIPY (Life Technologies, Grand Island, NY) staining and the other for LIVE/DEAD staining (Life Technologies, Grand Island, NY). Samples that were stained with BODIPY were submerged in $500 \mu \mathrm{L}$ of $4 \%(\mathrm{v} / \mathrm{v})$ paraformaldehyde (PFA) and incubated at $4^{\circ} \mathrm{C}$ overnight. Samples to be stained with the LIVE/DEAD kit were not incubated in 4\% PFA prior to staining.

\section{Collagenase digestion of murine and human adipose tissue}

Both murine and human tissue were digested at a concentration $1 \mathrm{~g}$ tissue $/ \mathrm{mL}$ of collagenase-containing digestion buffer consisting of $0.1 \%$ (weight/volume) collagenase Type I (Worthington Biochemical Corporation, Lakewood, NJ), 2.5\% (weight/volume) bovine serum albumin (Jackson ImmunoResearch West Grove, PA), 20 mM HEPES (Life Technologies, Grand Island, NY), 200 nM adenosine (Sigma,

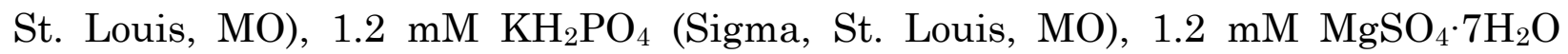
(Sigma, St. Louis, MO), 120 mM NaCl (Sigma, St. Louis, MO), 4.7 mM KCl (Sigma, St. Louis, MO), $1.3 \mathrm{mM} \mathrm{CaCl} 2 \cdot 2 \mathrm{H}_{2} \mathrm{O}$ (Fisher Scientific, Pittsburgh, PA) ${ }^{144}$. The murine tissues were divided into two control groups: untreated and mechanically dissociated only. Untreated murine tissue remained intact and was incubated in the same buffer above except without collagenase (collagenase-free buffer) for 60 minutes. Mechanically dissociated murine tissue was incised with scissors for one minute to simulate the effects of suction lipectomy in human tissue. Collagenasetreated murine tissue was also mechanically dissociated and subsequently 
enzymatically digested with collagenase-containing digestion buffer at increasing five minute intervals from $30-60$ minutes. Tissue samples were incubated in a $37^{\circ} \mathrm{C}$ water bath and agitated every 5 minutes to ensure uniform digestion. After digestion was complete, the tissues were washed three times with PBS to remove remaining collagenase based on previous studies ${ }^{235}$. Tissue samples were divided into two groups for each time point: one for BODIPY staining and the other for LIVE/DEAD staining. Samples to be stained with BODIPY were submerged in 500 $\mu \mathrm{L}$ of $4 \%(\mathrm{v} / \mathrm{v})$ paraformaldehyde (PFA) and incubated at $4^{\circ} \mathrm{C}$ overnight. Samples to be stained with the LIVE/DEAD kit were not incubated in 4\% PFA prior to staining.

Untreated human tissue harvested from suction lipectomy was incubated without collagenase buffer for 60 minutes. The treated human tissue was enzymatically digested with collagenase-containing buffer according to the same protocol outlined above for murine tissue.

\section{BODIPY Staining}

Samples were washed two times with PBS to remove residual 4\% PFA. $100 \mu \mathrm{L}$ of 10 $\mu \mathrm{g} / \mathrm{mL}$ BODIPY 558/568 $\mathrm{C}_{12}$ (Life Technologies D-3835)/Hanks Balanced Salt Solution (HBSS) was added to the samples and then incubated at $37^{\circ} \mathrm{C}$ for 20 minutes in the dark. Samples were washed three times with HBSS prior to mounting for imaging. 


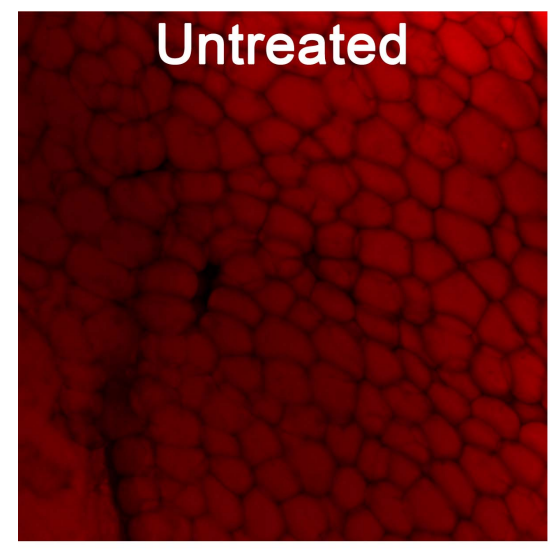

Time of collagenase digestion

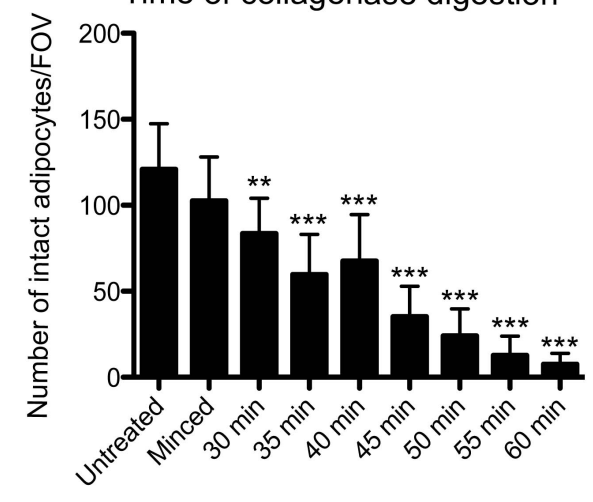

\section{0 minutes}

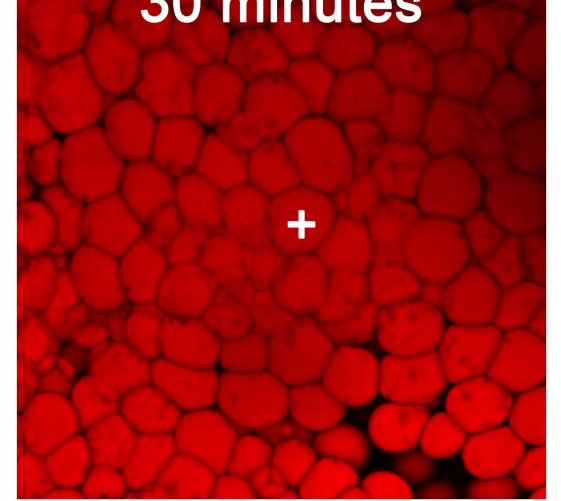

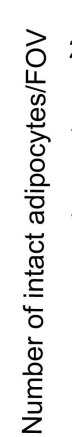

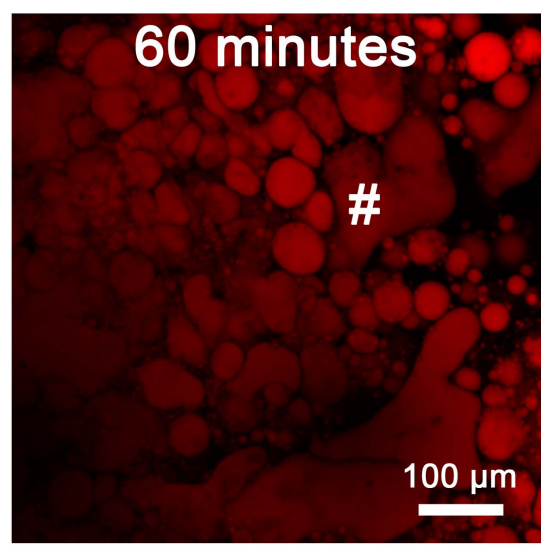

Time ex vivo

Figure 4.1: Viability of adipocytes in murine tissue

Murine inguinal fat pads were digested with collagenase at increasing five-minute intervals, stained with BODIPY and imaged using confocal microscopy. The "Untreated" fat pad was neither minced nor digested with collagenase. Also shown are representative images for collagenase digestion durations of 30 and 60 minutes. Left graph: Four trials $(n=4)$ using eight murine fat pads were quantified for intact adipocytes using three fields of view (FOVs) for each time point or control group. Confocal images and the quantification of intact adipocytes reveal that in murine inguinal fat pads, increasing the duration of collagenase digestion results in a decreased number of intact adipocytes when compared to untreated tissue (One-way ANOVA, Tukey's Post-hoc test, *: p-value $\leq 0.05^{* *}: p$ - value $\leq 0.01^{* * *}$ : p-value $\leq$ 0.001). Right graph: Three trials $(n=3)$ using six murine inguinal fat pads were quantified for intact adipocytes using three fields of view (FOVs) for each time point. Confocal images and the quantification of intact adipocytes reveal that increasing time after fat harvest does not have a statistically significant effect on the overall number of intact adipocytes in murine inguinal fat pads when compared to 20 minute ex vivo tissue (One-way ANOVA, Tukey's Post-hoc test). A representative intact adipocyte is marked with "+," and a representative lysed adipocyte is marked with "\#." Data shown are mean plus standard deviation. 


\section{LIVE/DEAD Staining}

After incubation $e x$ vivo or immediately following the wash steps after collagenase digestion, the LIVE/DEAD Cell Viability Kit (Life Technologies L7013) was used to stain tissue and assess cell viability. For murine tissue, Component $\mathrm{A}$ and Component B were diluted 1:500 in HBSS to make a working staining solution. For human tissue, Components A and B were diluted 1:250. $100 \mu \mathrm{L}$ of the working staining solution was added to each of the samples and tissue was incubated at room temperature for 30 minutes protected from light. Following staining, samples were washed three times with HBSS prior to mounting for imaging.

\section{Whole mounting tissue}

Samples were allowed to adhere to gelatin coated microscope slides for 5 minutes and sealed with coverslips in 50:50 PBS/glycerol solution.

\section{Confocal Imaging}

Samples were imaged on a Nikon TE 2000-E2 microscope (Nikon Instruments, Melville, NY) equipped with a Melles Griot Argon Ion Laser System (Melles Griot, Carlsbad, CA) and a Nikon D-Eclipse C1 confocal attachment. To account for tissue depth, $40 \mu \mathrm{m}$ Z-stacks were acquired with a $2 \mu \mathrm{m}$ step size. For both LIVE/DEAD and BODIPY samples, three, 200x fields of view were obtained. The entire field of view (FOV) was filled by adipocytes when imaging the BODIPY stained 
samples. FOVs with large vascular structures when imaging the LIVE/DEAD stained samples were excluded.

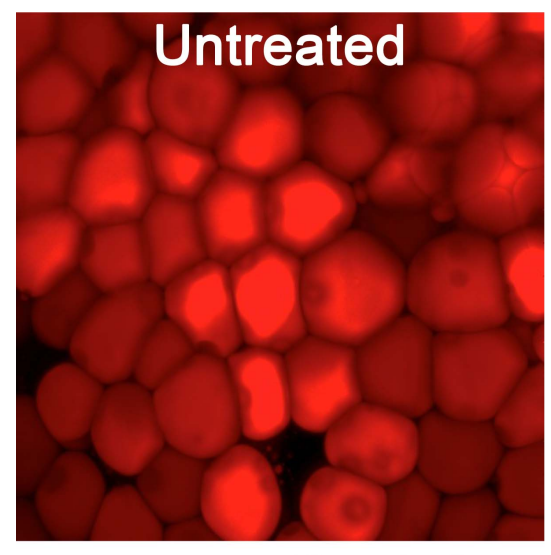

Time of collagenase digestion

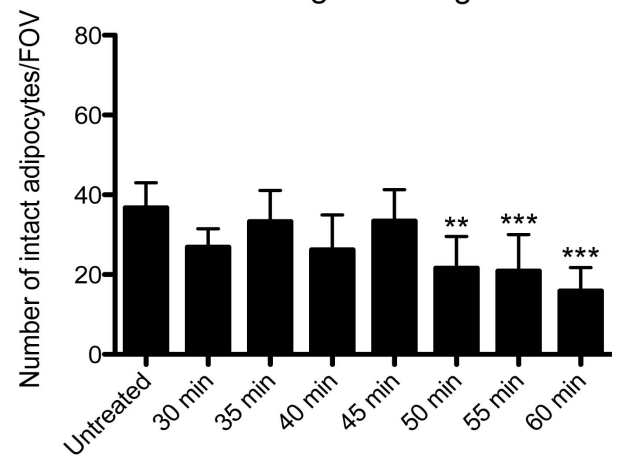

30 minutes
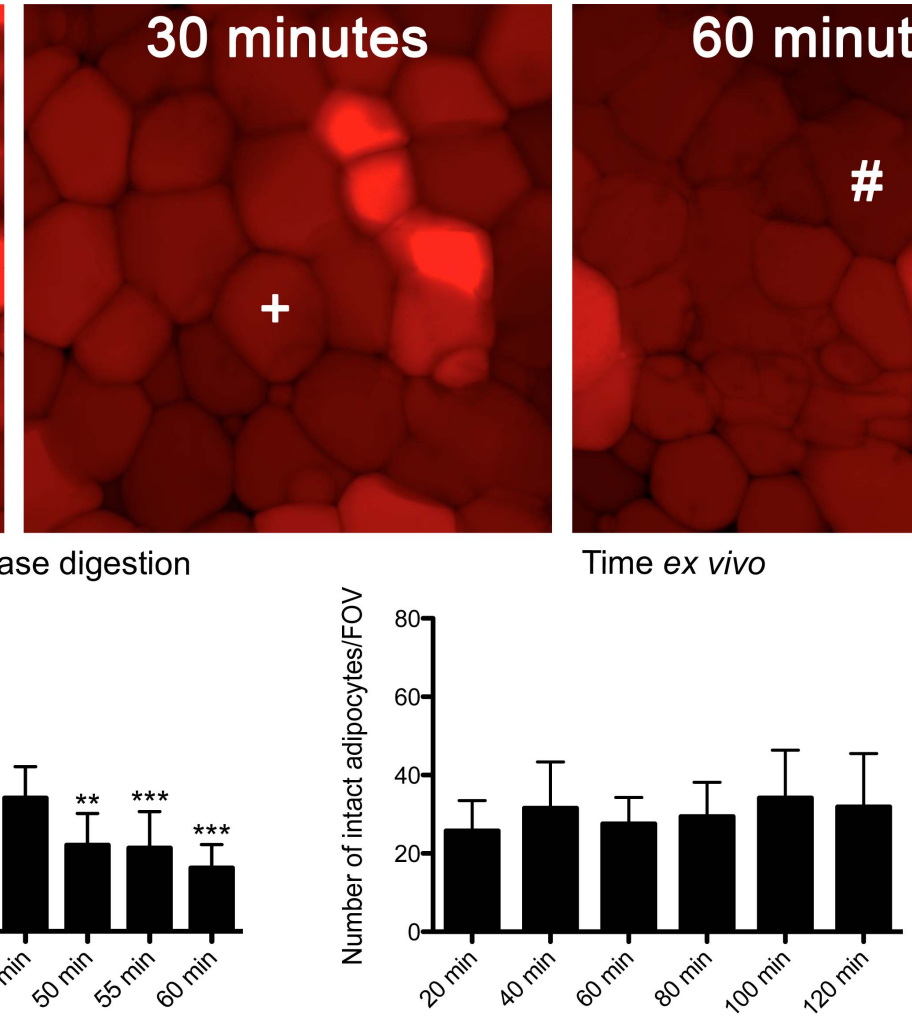

Figure 4.2: Viability of adipocytes in human lipoaspirate

Human lipoaspirate harvested using the Coleman technique was treated with collagenase at increasing five-minute intervals, stained with BODIPY and imaged using confocal microscopy. The "Untreated" fat pad was neither minced nor digested with collagenase. Also shown are representative images for collagenase digestion durations of 30 and 60 minutes. Left graph: Human lipoaspirate from three subjects $(n=3)$ were quantified for intact adipocytes using three fields of view (FOVs) for each time point or control group. Both confocal images and the quantification of intact adipocytes reveal that in human lipoaspirate, increasing the duration of collagenase digestion results in a decreased number of intact adipocytes when compared to untreated tissue (One-way ANOVA, Tukey's Post-hoc test *: p-value $\leq 0.05^{* *}$ :p-value $\leq 0.01^{* * *}:$-value $\left.\leq 0.001\right)$. Right graph: Human lipoaspirate from four subjects $(n=4)$ were quantified for intact adipocytes using three fields of view (FOVs) for each time point. Confocal images and the quantification of intact adipocytes reveal that increasing the duration of time up to 120 minutes after fat harvest does not have a statistically significant effect on the overall number of intact adipocytes in human lipoaspirate when compared to 20 minute ex vivo tissue. A representative intact adipocyte is marked with "+," and a representative lysed adipocyte is marked with "\#.” Data shown are mean plus standard deviation. 


\section{BODIPY Quantification}

BODIPY stains the lipids in adipocytes and allows visualization of these cells. Images acquired of BODIPY stained samples were examined with confocal microscopy to assess architectural integrity of adipocyte, and images were analyzed using ImageJ Software (National Institutes of Health, Bethesda, MD). The number of intact adipocytes for each image was counted by a single, trained and blinded observer. Adipocytes were considered intact if no lysis was evident, no lipid droplets were present and if the shape of the adipocyte was consistent with untreated adipose tissue.

\section{LIVE/DEAD Quantification}

Images acquired of LIVE/DEAD stained samples were analyzed using ImageJ software (National Institutes of Health, Bethesda, MD). Images acquired were split into the respective channels (red and green) and cells were counted manually by a single, trained and blinded observer. The quantity of red cells constituted the number of nonviable cells. The quantity of green cells constituted the total number of cells. Percent of viable cells were calculated using the following equation.

$$
\text { Percent of viable cells }=\frac{\text { Total cells }- \text { Dead cells }}{\text { Total Cells }} \times 100
$$

\section{Statistics}

A one-way ANOVA with a Tukey's Post-hoc test was used to compare all measurements and test for significance as appropriate with significance asserted at 
$p$-value $\leq 0.05$. For more details regarding statistical testing, please see figures and corresponding captions. 


\section{Results}

Duration ex vivo does not significantly affect adipocyte health in murine or human adipose tissue

Whole-mounted images of BODIPY stained murine and human tissue reveal no significant change in adipocyte viability as time ex vivo increases. The number of intact adipocytes did not change significantly for murine (Figure 4.1, bottom right) or human tissue (Figure 4.2, bottom right). Human adipocytes were larger in area than the murine adipocytes, consistent with previous findings ${ }^{236}$.

Duration ex vivo affects interstitial cell viability in both murine and human adipose tissue

Whole-mounted images of LIVE/DEAD stained murine adipose tissue reveal that the time ex vivo significantly affects the percentage of viable interstitial cells - as time is increased, the percentage of viable cells decreases. In murine fat grafts, after 20 minutes ex vivo, $80 \%$ of interstitial cells are viable and after 120 minutes $40 \%$ of interstitial cells are viable. Quantified numbers of murine cell viability are reported in bottom right panel of Figure 4.3. Images of LIVE/DEAD stained human tissue reveal the time ex vivo significantly affects the percentage of viable interstitial cells, albeit to a lesser degree in human tissue than murine tissue (Figures 4.3, 4.4). In human fat grafts, after 20 minutes ex vivo, $75 \%$ of interstitial cells are viable, and after 120 minutes $60 \%$ of interstitial cells are viable. Quantified numbers of human interstitial cells and the percentage that were viable are shown in the bottom right panel of Figure 4.4. 


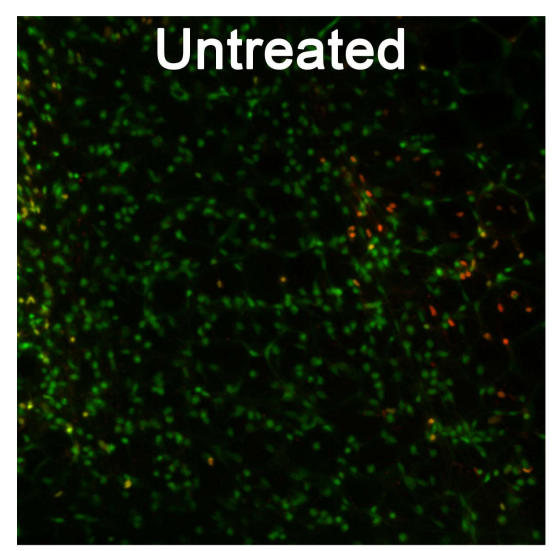

Time of collagenase digestion

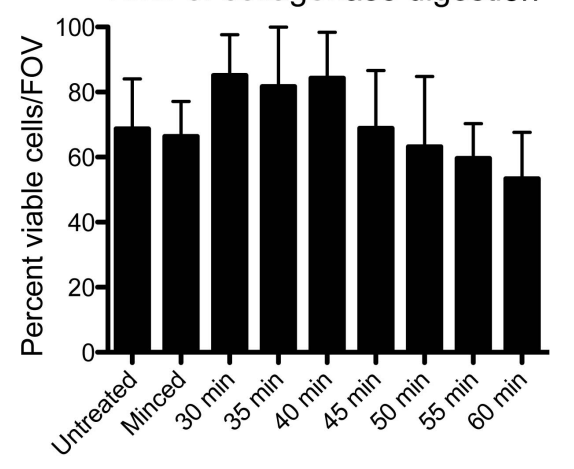

\section{0 minutes}

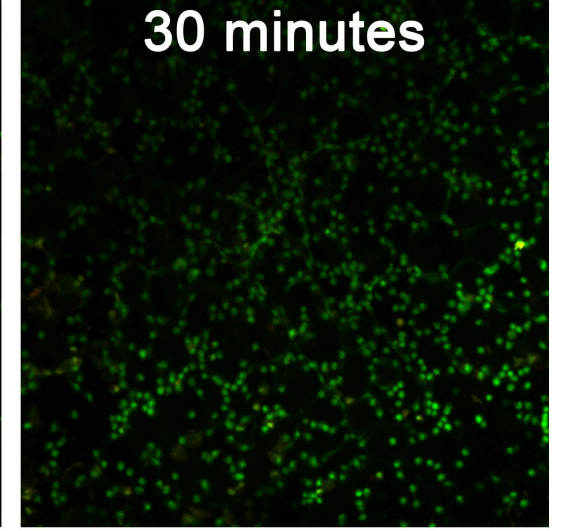

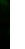

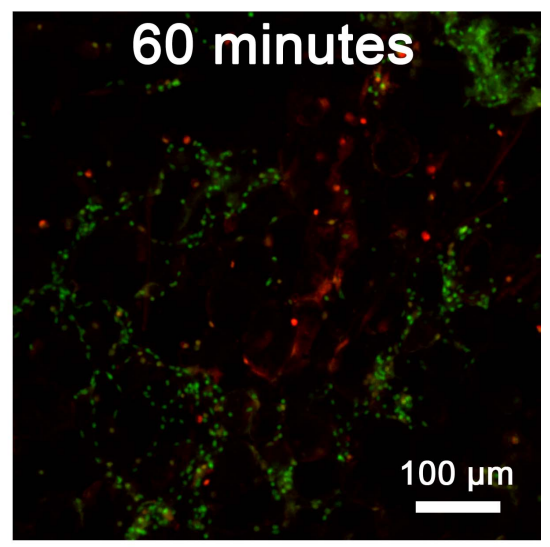

Time ex vivo

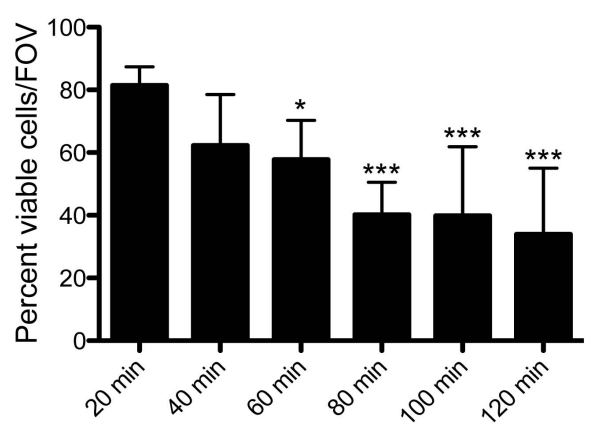

Figure 4.3: Viability of interstitial cells in murine tissue

Murine inguinal fat pads were treated with collagenase at increasing five-minute intervals, stained with LIVE/DEAD kit and imaged using confocal microscopy. Interstitial cells that are stained green are viable, and cells stained red or yellow are nonviable. The "Untreated" fat pad was neither minced nor digested with collagenase. Also shown are representative images for collagenase digestion durations of 30 and 60 minutes. Left graph: Four trials $(n=4)$ using eight murine fat pads were quantified for the percent of viable interstitial cells using three fields of view (FOVs) for each time point. Confocal images and the quantification of interstitial cells reveal that in murine inguinal fat pads, increasing the duration of collagenase digestion results in a decrease in the percentage of viable interstitial cells. Right graph: Three trials $(n=3)$ using six murine inguinal fat pads were quantified for the total number of interstitial cells using three fields of view (FOVs) for each time point. Confocal images and the quantification of interstitial cells reveal that in murine inguinal fat pads, increasing time after fat harvest results in a statistically significantly decreased percentage of viable interstitial cells when compared to 20 minute ex vivo tissue (One-way ANOVA, Tukey's Post-hoc test *: pvalue $\leq 0.05^{* *}:$ - value $\leq 0.01^{* * *}:$ p-value $\left.\leq 0.001\right)$. Data shown are mean plus standard deviation. 
Duration of collagenase digestion affects murine and human adipocyte viability

Collagenase digestion of murine adipocytes significantly decreases the number of intact adipocytes as the duration of collagenase digestion is increased (Figure 4.1). Mechanically dissociating the murine tissue (minced) resulted in a slight but not significant decrease in number of intact adipocytes. There is a significant decrease in intact adipocytes as collagenase digestion duration is increased in murine tissue. Similar to what was observed in murine adipose tissue, human adipose tissue exhibited a statistically significant decrease in the number of intact adipocytes as duration of collagenase digestion increased (Figure 4.2).

Duration of collagenase digestion affects murine and human interstitial cell viability Representative images of whole-mounted images of murine and human adipose tissue revealed that the amount of time that the tissue was exposed to collagenase digestion affected the viability of interstitial cells contained within the tissue (Figures 4.3, 4.4). Increasing the time required for collagenase digestion significantly decreased the percentage of viable interstitial cells in both murine and human adipose tissue. 

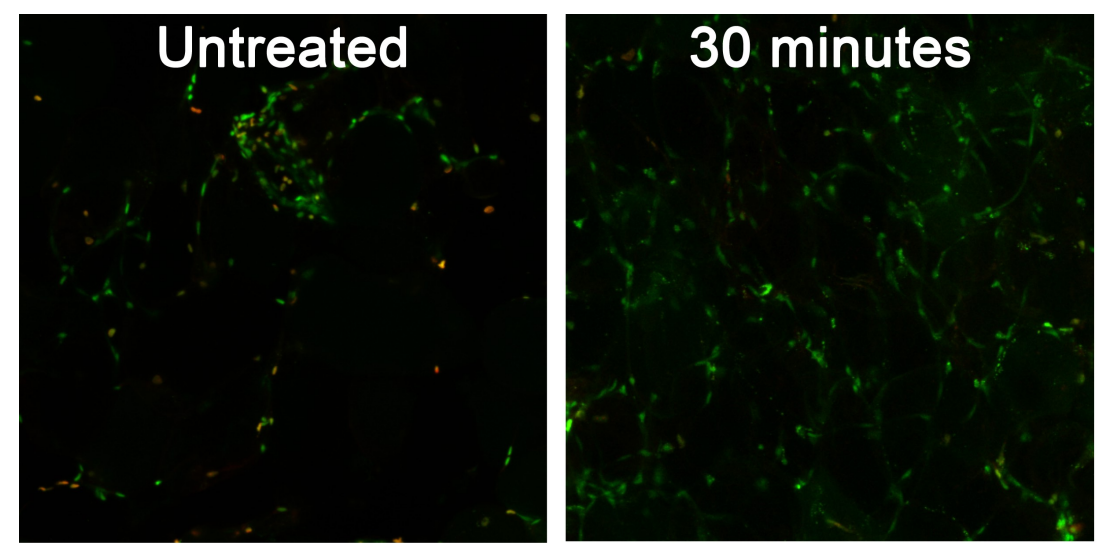

Time of collagenase digestion

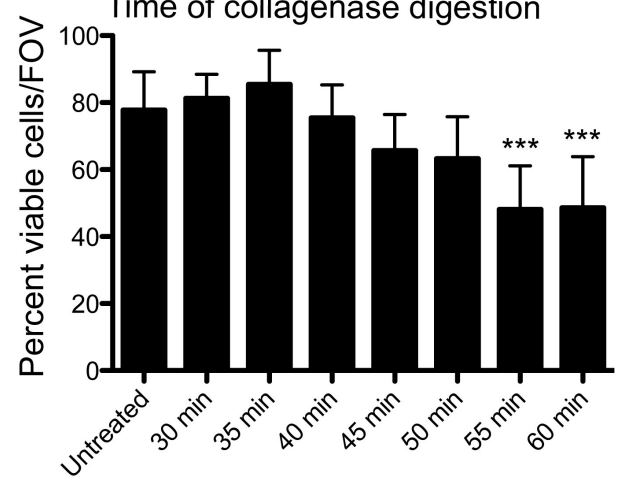

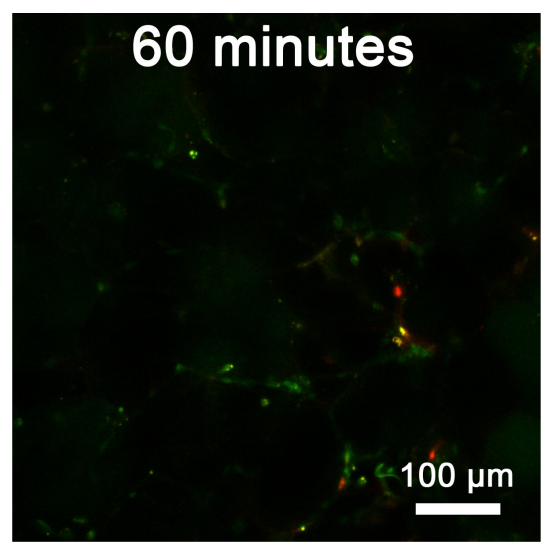

Time ex vivo

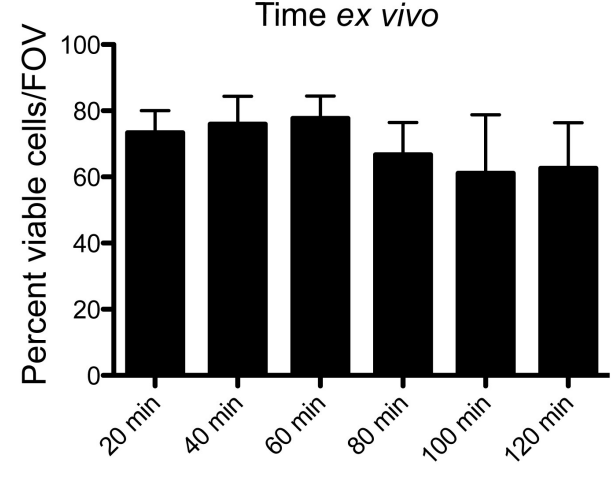

Figure 4.4: Viability of interstitial cells in human lipoaspirate

Human lipoaspirate harvested using the Coleman technique was treated with collagenase at increasing five-minute intervals, stained with LIVE/DEAD kit and imaged using confocal microscopy. Interstitial cells that are stained green are viable, and cells stained red or yellow are nonviable. The "Untreated" fat pad was neither minced nor digested with collagenase. Also shown are representative images for collagenase digestion durations of 30 and 60 minutes. Left graph: Human lipoaspirate from three subjects $(n=3)$ were quantified for the percent of viable interstitial cells using three fields of view (FOVs) for each time point. Confocal images and the quantification of interstitial cells reveal that in human lipoaspirate, increasing the duration of collagenase digestion results in a decreased percentage of viable interstitial cells when compared to untreated tissue (One-way ANOVA, Tukey's Post-hoc test *: p-value $\leq 0.05 * *$ : - value $\leq 0.01 * * *$ : p-value $\leq 0.001)$. Right graph: Human lipoaspirate from four subjects $(n=4)$ were quantified for the percent of viable interstitial cells using three fields of view (FOVs) for each time point. Confocal images and the quantification of interstitial cells reveal that in human lipoaspirate, increasing time after fat harvest does not result in a statistically significant difference in the percentage of viable cells when compared to 20 minute ex vivo tissue. Data shown are mean plus standard deviation. 


\section{Discussion}

Autologous fat grafting has increasing indications in both reconstructive and aesthetic plastic surgery. The benefits of fat grafting have been well described, however the conditions to improve retention of fat grafting are still being elucidated $6,8,218,220,221,223,224,237$. The aim of this study was to evaluate the effects of time and collagenase on human lipoaspirate and experimental mouse adipose tissue to optimize conditions affecting fat graft retention in clinical and research laboratory settings. Human lipoaspirate and murine adipose tissue were systematically examined using multiple modalities over increasing time ex vivo and over increasing collagenase digestion intervals. Human adipocytes and murine adipocytes remained intact over increased time intervals up to two hours. Murine and human interstitial cells within adipose tissue exhibited decreased viability over increased time to varying degrees. Murine interstitial cells were seemingly more affected by time ex vivo than human interstitial cells. Increased collagenase digestion time significantly decreased viability of both human and murine adipocytes and interstitial cells.

Determining the effect of increasing temporal delays after harvest of the graft and prior to injection of the fat graft was a goal of this present study, which used multiple modalities to quantify viability of cell populations within adipose tissue. The finding that adipocytes (human and murine) are resilient enough to remain viable two hours ex vivo is consistent with findings by Eto et al. ${ }^{238}$. Specifically in a human fat graft, the adipocytes and the cells in the interstitial space, including endothelial cells and adipose derived stem cells, have been found previously to 
maintain viability one day after removal and when maintained in serum-free medium under hypoxic conditions ${ }^{238}$. We report that adipose tissue (human and murine) viability is not significantly impaired up to 2 hours when maintained in saline solution at room temperature, which we feel is more akin to a surgical setting or laboratory setting. Our findings agree with the findings of Eto et al. and build on their findings, potentially providing more insight into the survival of adipose tissue in a clinical or laboratory setting. Temporal delays after harvesting murine adipose tissue for pre-clinical experiments may be substantial, but the viability of murine adipocytes was not significantly attenuated over time in this study.

In our ex vivo studies, the interstitial cell component of the adipose tissue harvested was affected differently in both species studied. The human interstitial cells were resistant to the effect of increased time ex vivo, while the murine interstitial cells decreased in viability with increased time ex vivo. This may be in part explained by the technical differences in the treatment of the murine fat graft (manual dissociation required to simulate liposuction) as compared to the human fat graft (harvested via traditional liposuction). Further study into this is required to understand the differences in murine interstitial cells compared to human interstitial cells, as this is the first report of this observed difference interstitial cell viability between different species with respect to time.

Results revealed that increased duration of collagenase digestion significantly decreased adipocyte and interstitial cell viability in both human and murine populations. This is consistent with prior work by Piasecki and colleagues ${ }^{239}$ 
who found that human fat grafts fragmented at higher rates with increased duration of collagenase digestion with times ranging from $0-180$ minutes ${ }^{239}$. No other studies to date have examined the effect of collagenase digestion duration on murine adipocytes and murine interstitial cells. Although our studies were carried out in two different species (murine and human), the results for each species are valuable to the scientific and surgical communities, as pre-clinical murine models play a critical role in the therapy development pipeline.

Our data show that collagenase may be detrimental to interstitial cell viability and adipocyte integrity and that it is therefore very important to deactivate or wash out any residual collagenase prior to enhancing fat grafts with SVF or using adipose tissue for preclinical studies. Failure to do so may lead to unwanted fat necrosis, a false sense of correction of the deformity intraoperatively, and a low rate of retention of the fat graft in the postoperative period.

It is imperative to consider and tailor the time, temperature, concentration, and type of collagenase used for specific studies or experimental trials. Bacterialderived collagenase remains the most common proteolytic enzyme used for tissue dissociation $^{240}$ and has growing implications in the plastic surgery and reconstructive field for use in adipose tissue. Collagenase disrupts the collagen-rich extracellular matrix of adipose tissue allowing for easy harvest of interstitial cells and single adipocytes.

"Crude" collagenases (several types exist) are often used and are not pure collagenase, but rather a mixture of several enzymes in addition to collagenase 
capable of dissociating the ECM. For the present studies, type I collagenase was used. This is typically used for adipose dissociation but there is no standard in the literature, and other authors have utilized other collagenase types for dissociation. Also important in the collagenase digestion process is the temperature and collagenase concentration used, with varying temperatures and concentrations used in the literature. Although our studies used one type of collagenase (Type I), one temperature for digestion $\left(37^{\circ} \mathrm{C}\right)$, and one concentration $(1 \mathrm{mg}$ collagenase $/ \mathrm{mL})$, they highlight the importance and the possible deleterious effects of collagenase on the adipocytes and interstitial cells in both human and murine tissue.

Currently, the use of collagenase to isolate SVF and enhance fat grafts is not an approved practice by the United States Food and Drug Administration (FDA) but is practiced in other countries ${ }^{8,223}$. Further, collagenase as a biologic has been recently approved by the FDA (XIAFLEX ${ }^{\circledR}$, Auxilium Pharmaceuticals) to treat Dupuytren's contracture ${ }^{241}$ and Peyronie's disease ${ }^{242}$. With proper characterization and future studies to examine the effect of collagenase in more detail, FDA approval to enhance fat grafts with collagenase-isolated SVF may be imminent.

A few specific limitations temper the conclusions drawn from this study. First, mice lack sufficient volumes of subcutaneous adipose tissue to perform liposuction in vivo, thus inguinal fat pads were manually dissociated as previously shown to adequately fragment the fat for the purposes of study ${ }^{243}$. This surgical technique is well established as an adequate proxy for liposuction; however, it may increase interstitial cell susceptibility to cell death. Additionally, the small sample 
sizes also limit the conclusions that can be drawn, however the evidence of parallel findings consistent across multiple modalities in this study add strength to the conclusions. All murine tissue was treated in the same manner and thus serves as an internal control. Secondly, we used architectural integrity after BODIPY staining as a marker for viable adipocytes (no lysis evident, no lipid droplets present, shape consistent with untreated adipose tissue). Because our chosen assays rely on architectural integrity, it is possible that only cells with severely compromised membranes are being detected as non-viable. There may be a subpopulation of cells with irreversible damage but do not have compromised membranes and thus we may be underestimating the percent of non-viable cells. Reliably determining a viable, intact adipocyte from a nonviable adipocyte has proven to be a challenge that has been identified by the literature, and it is necessary to employ a combination of viability assays or stains. Suga et al. ${ }^{244}$ conclude that three common viability assays (XTT, MTT, trypan blue) cannot be used singly and must be employed in a combinatorial fashion for adipocyte specificity. Suga et al. ${ }^{244}$ used Hoechst 33342 and propidium iodide to distinguish viable, intact adipocytes following collagenase digestion. We coupled our BODIPY staining with a LIVE/DEAD stain to similarly provide a global view of overall adipose tissue viability that is consistent with their use in other published studies ${ }^{25,245,246}$. The combination of these assays and the consistencies of the findings from each assay adds to the strength of the conclusions that can be drawn. Our studies were conducted using liposuction samples from all female patients 
potentially limiting the generalizability of the findings to both sexes. However, this is consistent with published national trends showing that $89 \%$ of patients undergoing autologous fat grafting in 2013 were female ${ }^{247}$. Although this limits the generalizability of the findings in this study to the female gender, most patients undergoing autologous fat transfer nationally are female thus the findings are highly relevant. Further, there are several examples in the literature outlining the potential impact of donor gender on ASC behavior such expansion and differentiation capabilities. Aksu et al. showed that male-derived human ASCs differentiate down an osteogenic pathway more effectively ${ }^{248}$, while Ogawa et al. showed that female murine ASCs differentiate down the adipogenic pathway more effectively ${ }^{249}$. To our knowledge, no studies have been conducted examining the viability of the interstitial cells or adipocytes between male and female donors. Our studies do not characterize the cell type composition of the interstitial cell compartment and do not examine the individual cell types. However, these future studies are feasible and would be interesting to couple these findings with an in vivo retention model of fat grafting (e.g. dorsal subcutaneous implantation) to correlated specific cell types responsible for fat graft survival.

Our approach provides insight into both the interstitial cells within the adipose tissue, as well as the adipocytes themselves. We are confident that these two staining protocols allow us to differentiate healthy and viable adipose tissue from adipose tissue that has been detrimentally affected. Altering the collagenase concentration, the temperature in which the digestion is performed, and the type of 
collagenase will very likely affect both clinical and research study outcomes. Thus at the present time, while we are not able to definitively identify an "optimal" digestion time as it is likely dependent on these aforementioned parameters; our results provide insight into the detrimental effects of increasing collagenase digestion duration. 


\section{Conclusions}

Our results suggest that adipose tissue can withstand an ex vivo time period of up to two hours with no significant effect on adipocyte viability. This information can inform guidelines for operating procedures and provide surgeons with a timeframe for fat grafting that ensures cell viability and may lead to better engraftment outcomes and volume retention. Autologous fat grafting is frequently performed in the setting of larger-volume suction lipoplasty, which would require the harvest of more adipose tissue and delay the surgical time to grafting. Our data suggest that surgeons may proceed without fear of loss of graft viability from this temporal delay of up to two hours.

While our study was not designed to identify an optimal digestion duration that would preserve adipocyte and interstitial cell viability while allowing for complete dissociation of adipose tissue for isolating of SVF, our findings show that prolonged collagenase digestion decreases the number of viable interstitial cells and adipocytes in murine and human tissue. Our study allows us to conclude that increased collagenase digestion duration may be detrimental to cell viability and not likely beneficial for autologous fat grafting. 


\section{Chapter 5}

\section{EFFECTS OF COLLAGENASE DIGESTION AND SVF SUPPLEMENTATION ON VOLUME RETENTION OF FAT GRAFTS}

Acknowledgements: Jonathan B. Olenczaka ${ }^{a}$ Catherine E. Davis ${ }^{b}$, Lisa S. Salopek ${ }^{a}$, Angela Pineros-Fernandez ${ }^{a}$, Kant Y. Lina, Shayn M. Peirce ${ }^{a, c}$, Patrick S. Cottler ${ }^{a}$

${ }^{a}$ Department of Plastic Surgery, University of Virginia ${ }^{b}$ Department of Biology, University of Virginia

${ }^{c}$ Department of Biomedical Engineering, University of Virginia 


\section{Abstract \\ Background}

The use of autologous fat as a soft tissue defect filler has increased over the past decade in reconstructive and aesthetic surgeries. Enhancement of autologous fat grafts with SVF has been reported to improve long-term volume retention. SVF is most commonly isolated using enzymatic digestion and it is unknown what effect this digestion has on the adipocytes and SVF cells that comprise the graft. Previous studies by our group found that increasing collagenase digestion duration detrimentally affected the viability of adipocytes and SVF cells. Here, we aim to determine if collagenase digestion of adipocytes prior to grafting is detrimental to graft retention and if SVF supplementation can abrogate these potential deleterious effects.

\section{Methods and Results}

Here, we utilize a published xenograft model in which human lipoaspirate was implanted above the scalps of immunocompromised mice to study the effect of collagenase digestion on in vivo graft survival after 12 weeks. We used four experimental groups: grafts comprised of collagenase-digested and non-digested adipocytes (50 minute digestion) and grafts with and without SVF supplementation. We used micro-CT to non-invasively quantify the volume of the graft, in conjunction with $H \& E$ staining of histological cross-sections of implanted and excised grafts to assess overall tissue health. We found that adipocytes that were collagenasedigested prior to implantation had significantly lower retention rates at 12 weeks and had a lower quality of tissue health. Further, we found that SVF 
supplementation of the digested grafts improved graft survival, but not to the level observed in undigested grafts.

\section{Conclusions}

We conclude that collagenase digestion adversely affects the long-term volume retention of fat grafts, but graft retention is ameliorated by SVF supplementation. This pre-clinical study can serve as a template for the design of clinical studies to further clarify the efficacy and safety of using collagenase-digested fat grafts and SVF in clinical fat grafting. 


\section{Introduction}

The use of autologous fat has increased in both reconstructive and aesthetic plastic surgery cases over the past decade ${ }^{4,250}$. Autologous fat provides a means to repair and fill soft tissue defects ranging from small-volume contour irregularities of the face to large-volume breast and buttock reconstructions $218,221,228,251$. Benefits of using autologous fat are the relative ease of harvest, the abundance of tissue available to surgeons, innate biocompatibility, and the avoidance of using a synthetic or foreign implant material ${ }^{3-5}$. While the use of autologous fat in the clinic is rising, the long-term retention rate of grafted autologous fat is still unpredictable, with resorption rates ranging from $25-80 \%{ }^{5}$.

While the mechanism(s) responsible for fat graft survival remain unclear, proper vascularization and subsequent sufficient oxygen/nutrient delivery appear to be the crux of fat graft survival156,251-253. Clinicians and scientists have aimed to improve the long-term retention rate of fat grafts, and these approaches have been the subject of many studies over the past decade. Central to most of these studies is the "enhancement" of fat grafts with the stromal vascular fraction (SVF), or more specifically, adipose-derived stem cells (ASCs) ${ }^{8,254,255}$. The SVF, a compartment of adipose tissue that is easily isolated from lipoaspirate, contains preadipocytes, ASCs, endothelial progenitor cells, macrophages, and vascular cells ${ }^{21,22}$. It is believed that the progenitor and stem cells within the SVF form the basis of better fat graft retention after implantation by providing a source for new adipocytes, vascular cells, and support cells in the grafted tissue ${ }^{23,256}$. The SVF is most commonly isolated from the adipose tissue using an enzyme ${ }^{257}$ such as collagenase ${ }^{24}$ 
or an enzymatic "cocktail", which contains various collagenases (i.e. Liberase Blendzyme ${ }^{258}$ ). Less common and less effective techniques used to isolate SVF include centrifugation ${ }^{25}$, mechanical isolation via vigorous shaking ${ }^{26}$, and decanting after density gradient separation ${ }^{27}$. While the enhancement of fat grafts with SVF is a commonly practiced technique, the treatment of the fat grafts prior to implantation, specifically the adipocyte portion, is not a standardized practice. Herein, we focus on fat grafting techniques that utilize enzymatic digestion, specifically collagenase, to harvest SVF and enhance fat grafts prior to grafting.

Previous studies have reported the use of enzymatic digestion of lipoaspirate material prior to using the SVF and adipocytes as part of the autologous graft. Typically, the enzymatically isolated SVF is added to adipocytes that have not been enzymatically digested (reserved portion of lipoaspirate) ${ }^{8,259}$, but reports in other countries have described the use of collagenase-digested adipocytes as part of the graft 7,260 . Youn et al. used collagenase-digested adipocytes to surgically correct infraorbital dark circles ${ }^{260}$. Youn et al. cite that collagenase digestion of the adipose tissue increased the contouring ability of the fat graft by making it a "more liquid filler" and reported safe and efficacious results ${ }^{260}$. While the use of SVF/adipocytes harvested from collagenase-digested lipoaspirate is not currently approved for use by the United Stated Food and Drug Administration (FDA), collagenase has been recently approved for the treatment of Dupuytren's contracture ${ }^{261}$ and Peyronie's disease $^{262}$ in the United States. Furthermore, with the increasing use of enhanced fat grafting specifically in large volume reconstructions and in cases of patients 
with low adiposity, it will be important to study the effect of collagenase digestion on the adipocytes. During cases of large volume reconstructions and in patients with low adiposity, it is may be necessary to use all of the fat taken from the patient and not to discard potentially useable fat for reconstruction. Future studies conducted on collagenase-digested adipocytes and SVF may provide grounds for FDA approval of collagenase-isolated SVF and adipocytes in fat grafts.

In our previously published studies ${ }^{263}$ described in Chapter 4 , we report the in vitro effect of collagenase digestion on adipocytes and SVF for both human and murine adipose tissue. We found that increasing the duration of collagenase digestion is detrimental to both the SVF and the adipocytes, causing decreases in the viability of both interstitial cells (which comprise the SVF compartment) and adipocytes. For both adipocytes and interstitial cell viabilities, the effect of collagenase digestion was gradual with a "threshold" of tolerance (Figures 4.1-4.4). For human tissue, the viability of adipocytes and SVF was significantly decreased at 50 minutes of digestion (Figures 4.2 and 4.4). While these in vitro studies were the first to indicate the potentially detrimental effects of collagenase digestion on SVF and adipocytes, it is imperative to consider the effect of collagenase digestion on in vivo graft survival. To our knowledge, no studies to date have examined the effect of collagenase digestion on in vivo graft survival.

In this study, we utilized a previously published xenograft model ${ }^{141}$ in which we implanted human fat grafts above the scalps of immunocompromised mice to study the effect of collagenase digestion on in vivo graft survival. We employed the 
use of X-ray microtomography (micro-CT) to non-invasively track the volumes of implanted fat grafts over the course of 12 weeks. We coupled micro-CT data with H\&E staining to provide a unique outlook at the overall tissue health and viability. Our findings suggest that adipocytes that were collagenase digested prior to grafting had significantly lower retention rates and were nearly undetectable at the 12 week terminal time point. Collagenase-digested grafts had a lower quality of tissue health, as evidenced by $\mathrm{H} \& \mathrm{E}$ histological scoring. Further, our findings suggest that SVF supplementation of the digested grafts improved graft survival, but not to a level we observed in undigested grafts.

A better understanding of the impact of collagenase digestion on in vivo graft survival is of importance to clinicians and will help clarify the efficacy of using digested adipocytes/SVF during autologous fat grafting. This study builds on our in vitro findings and aims to determine the potential detrimental effect on in vivo graft retention. 


\section{Materials and Methods}

Human adipose tissue harvest

All human tissue was obtained in accordance with The University of Virginia Institutional Review Board. Human subcutaneous adipose tissue was acquired from two patients undergoing elective intraoperative suction lipectomy performed by the Department of Plastic Surgery at The University of Virginia. Both patients were nondiabetic female, ages 36 and 42, with body mass indexes of 32.0 and 34.0 respectively. After removal from the body, the lipoaspirate was incubated in a sealed, sterile container at room temperature $\left(25^{\circ} \mathrm{C}\right)$ until use.

\section{Isolation of adipocytes from collagenase-digested human adipose tissue}

Human adipose tissue was digested in a collagenase-containing buffer as described previously in Chapter 4 and in previous studies ${ }^{144,263}$. Briefly, adipose tissue was digested for at a concentration of gram of tissue per milliliter of collagenasecontaining buffer consisting of $0.1 \%$ (weight/volume) collagenase Type I, 2.5\% (weight/volume) bovine serum albumin, $20 \mathrm{mM}$ HEPES, $200 \mathrm{nM}$ adenosine, $1.2 \mathrm{mM}$ $\mathrm{KH}_{2} \mathrm{PO}_{4}, 1.2 \mathrm{mM} \mathrm{MgSO} \cdot 7 \mathrm{H}_{2} \mathrm{O}, 120 \mathrm{mM} \mathrm{NaCl}, 4.7 \mathrm{mM} \mathrm{KCl}, 1.3 \mathrm{mM} \mathrm{CaCl} \cdot 2 \mathrm{H}_{2} \mathrm{O}$. Samples were incubated in a $37^{\circ} \mathrm{C}$ water bath under constant agitation for 50 minutes. After digestion was complete, the samples were washed with PBS three times for five minutes each wash to remove residual collagenase. After PBS washes, samples were centrifuged at 1100 RPM for five minutes and the adipocytes were isolated using a micropipette. These adipocytes were termed "collagenase-digested" 
adipocytes and were stored in sterile microcentrifuge tubes at room temperature until use. All procedures were performed in a biosafety cabinet to maintain sterility.

\section{Isolation of adipocytes from untreated human adipose tissue}

Adipocytes were also derived from undigested adipose tissue in the same way as above but were incubated in collagenase-free buffer for 50 minutes. After incubation in the collagenase-free buffer, samples were washed three times with PBS as stated above and were centrifuged at 1100 RPM for five minutes. The "untreated" adipocytes were isolated carefully by using a micropipette and were stored in sterile microcentrifuge tubes at room temperature until use.

\section{Stromal vascular fraction isolation}

After the "collagenase-digested" adipocytes were removed via micropipette as stated above, the remaining portion of the sample was used to isolate the stromal vascular fraction (SVF) for subsequent studies. After centrifugation, the sample stratifies into three layers based on densities - the top layer is the adipocytes (used as stated above), the middle layer is the tumescent fluid and washing fluid, and the bottom layer contains the stromal vascular fraction and blood cells. The adipocytes (top layer) were removed for use and the middle layer was aspirated leaving the bottom pellet containing the SVF. $1 \mathrm{~mL}$ of DMEM/F12 media was added to the pellet and gently mixed by pipetting. The solution was centrifuged at $1100 \mathrm{RPM}$ for five minutes and $500 \mu \mathrm{L}$ was removed from the sample. $1 \mathrm{~mL}$ of erythrocyte lysis buffer 
(eBioscience) was added and the solution was gently vortexed to resuspend the pellet. The sample was incubated for five minutes at room temperature, which was followed by the addition of $1.5 \mathrm{~mL}$ of $\mathrm{DMEM} / \mathrm{F} 12$. This solution was sterile filtered through a $40 \mu \mathrm{m}$ centrifuge tube mesh and centrifuged at 1100 RPM for five minutes. After centrifugation, the supernatant was removed and the pellet was resuspended in $1 \mathrm{~mL}$ of DMEM/F12. The cells were counted using a hemocytometer and were resuspended in sterile PBS into a known volume for the concentration desired for the subsequent studies.

\section{Preparation of adipose grafts}

Four separate groups were used for these studies: 1.) undigested adipocytes without SVF supplementation, 2.) undigested adipocytes with SVF supplementation, 3.) digested adipocytes without SVF supplementation, and 4.) digested adipocytes with SVF supplementation (Figure 5.1A). All implanted adipose tissue grafts were 250 $\mu \mathrm{L}$ and grafts that were SVF supplemented contained $12,500 \mathrm{SVF}$ cells $/ 250 \mu \mathrm{L}$ of fat based on findings from a previous paper that titrated the concentration of SVF cells in fat grafts ${ }^{254}$. Grafts were prepared based on the groups above and were loaded into $1 \mathrm{~mL}$ syringes coupled to a sterile 16 gauge catheter tubing for injection.

\section{Implantation of adipose graft implants}

A xenograft adipose tissue graft model was modified in which adipose grafts were implanted above the scalp of recipient mice ${ }^{141}$. NOD SCID mice were anesthetized 
with $2 \%$ isoflurane and the hair on the top of head and around the ears were shaved and depilated. Following depilation, the area was sterilized with three alternating ethanol and povidone-iodine wipes. A small incision $(\sim 1 \mathrm{~cm})$ was made at the base of the skull with sterile surgical scissors and the fascia and skin was undermined with forceps to make a "pocket" for implantation of adipose graft. The adipose graft (250 $\mu \mathrm{L}$ ) was injected slowly through the 16-gauge catheter tubing and special consideration was taken to ensure that no adipose tissue emanated from the incision after injection. The incision was closed with 6-0 nylon suture.

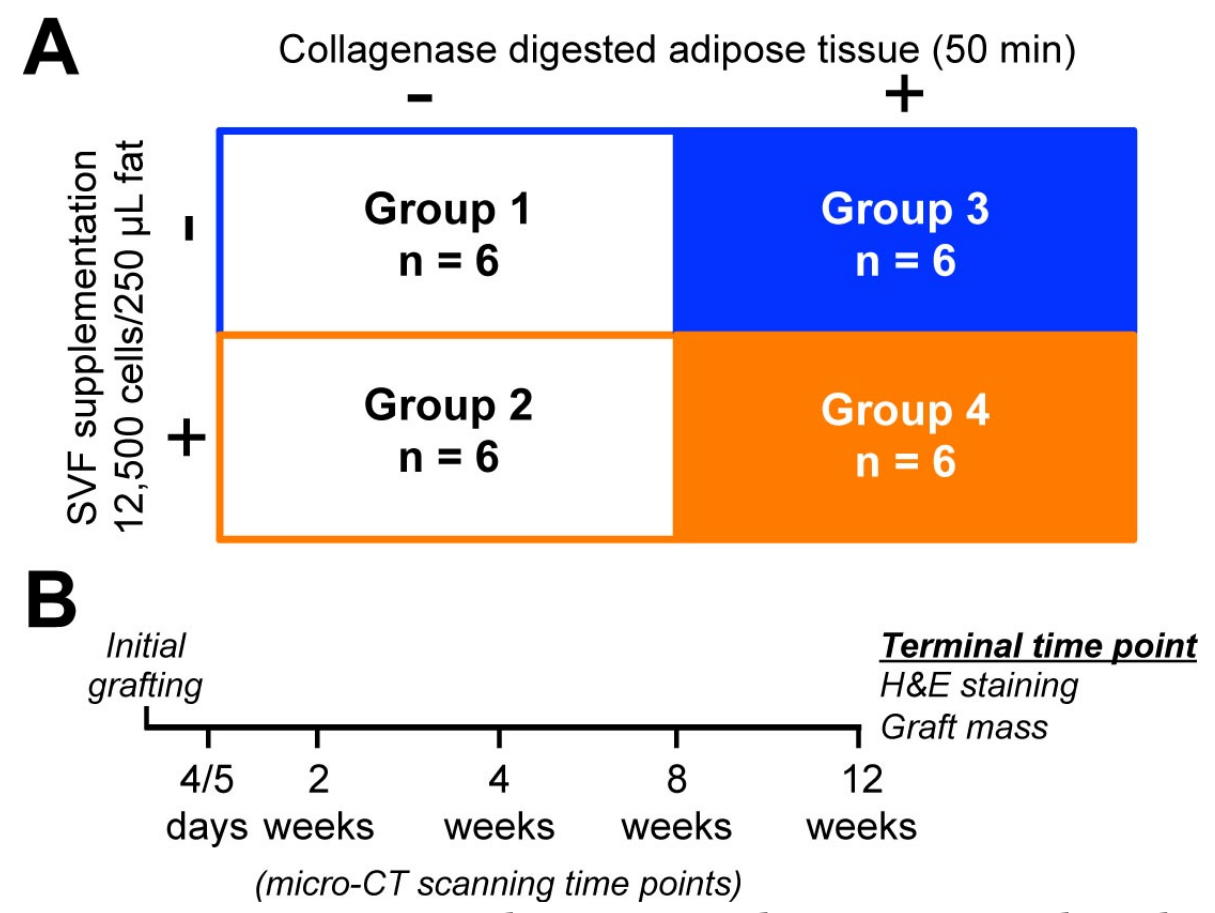

Figure 5.1: Experimental groups and experimental outline A.) Experimental Groups 1-4 consisting of collagenase-digested and undigested adipocytes with or without SVF supplementation. B.) Experimental timeline depicted micro-CT scanning time points and terminal time point. 
Micro computed tomography scanning of grafts

A Scanco vivaCT 40 micro-CT instrument (Scanco Medical) was used to scan recipient mice at various time points after implantation of human adipose tissue grafts as depicted in Figure 5.1B. micro-CT scanning allowed for non-invasive quantification of the graft volume and provided topographical information of the adipose graft. Mice were anesthetized with $2 \%$ isoflurane prior to and during the entirety of the scanning procedure. Settings used on the micro-CT instrument were as follows: tube voltage $-55 \mathrm{kV}$, tube current - $145 \mathrm{pA}$, power $-8 \mathrm{~W}$, integration time $-316 \mathrm{msec}$, and voxel size $-70 \mu \mathrm{m}$. All mice were scanned four or five days postimplantation, two weeks post-implantation, four weeks post-implantation, eight weeks post-implantation, and twelve weeks post-implantation (Figure 5.1B).

\section{Harvest of adipose tissue grafts}

At the terminal time point of twelve weeks post-implantation, mice were euthanized via $\mathrm{CO}_{2}$ asphyxiation. The skin above the scalp was carefully excised and the adipose graft was exposed. An image of the adipose graft was taken with a handheld camera to provide a macroscopic view of the graft. After imaging, the adipose graft was carefully excised from the skin and the mass of the graft was recorded. Adipose tissue was stored in $4 \%$ paraformaldehyde at $4^{\circ} \mathrm{C}$ for seven days to allow for complete fixation. In some recipient mice (Group 3), the adipose graft was undetectable and was not harvested. 


\section{Analysis of acquired micro-CT scans}

Fiji, an image processing package based on ImageJ, was used to process the DICOM files generated from the micro-CT scans. "Segmentation Editor", a plugin within Fiji, was used to analyze the DICOM files and to quantify the graft volume. The DICOM files were imported into Fiji as an "Image Sequence" and converted to 8-bit grayscale (Figure 5.2A). The imported stack of images was loaded into "Segmentation Editor" and the freehand tracing tool was used to outline the adipose graft on every fifth slice until the adipose graft was no longer visualized (Figure 5.2B). The adipose graft was distinct from the surrounding skin based on the color and contrast of the image. After tracing every fifth slice, interpolation was performed to create selections/traces for slices between the previously traced slices. A 3D projection of these slices was constructed and the volume of the adipose tissue graft was calculated after setting the voxel size for scaling purposes (Figure $5.2 \mathrm{C}, \mathrm{D})$.

\section{$H \& E$ staining and scoring}

After fixation, adipose grafts were processed for paraffin embedding by the University of Virginia Research Histology Core. Samples were cut $5 \mu \mathrm{m}$ thick and were stained with hematoxylin and eosin (H\&E) stain. Individual 100X images were acquired using an EVOS XL microscope (Life Technologies) and montages were made of the entirety of the adipose graft for analysis. Montaged images were scored by three blinded, trained observers on a scale of 0-5 (0: non-existent, 5 : significant 
presence) and were evaluated for the presence of inflammation, cysts/vacuoles, membrane integrity, and fibrosis, as a combinatorial metric of tissue health as described previously ${ }^{255,264}$. Representative examples of fibrosis, cysts/vacuoles, and inflammation are depicted in Figure 5.3A-C as well as average scores in Figure 5.3D. 


\section{Results}

Collagenase digestion of adipocytes prior to implantation decreases volume retention Calculation of adipose graft volumes from 3D projections revealed that collagenase digestion of adipocytes prior to grafting significantly reduced and detrimentally affected volume retention post-implantation. The calculated graft volume of the collagenase treated group with SVF supplementation (Group 4, $83.90 \mathrm{~mm}^{3}$ ) was roughly one-third of the initial grafted volume $\left(250 \mathrm{~mm}^{3}\right)$ after only four/five days post-implantation (Figure 5.2D). The group that was collagenase treated without SVF supplementation (Group 3) was nearly undetectable via micro-CT scanning after four/five days with an average calculated volume of only $0.87 \mathrm{~mm}^{3}$. The groups containing adipocytes that were not collagenase digested prior to implantation (Groups 1 and 2) had much higher calculated volumes immediately postimplantation (Figure 5.2D). The group with undigested adipocytes without SVF supplementation (Group 1) had an average volume of $192.06 \mathrm{~mm}^{3}$, while the group with undigested adipocytes with SVF supplementation (Group 2) had an average volume of $163.21 \mathrm{~mm}^{3}$ (Figure 5.2D).

This observed decrease in calculated graft volume for digested adipocytes was observed for all time points post-implantation up to twelve weeks. At the terminal time point of twelve weeks, the collagenase-digested group without SVF (Group 3) was undetectable and the collagenase-digested group with SVF supplementation (Group 4) had an average volume of $4.98 \mathrm{~mm}^{3}$. The undigested group without SVF had an average calculated volume of $109.69 \mathrm{~mm}^{3}$ while the undigested group with SVF had an average calculate volume of $86.01 \mathrm{~mm}^{3}$ (Figure 5.2D). Based on these 
data, it is apparent that collagenase digestion prior to grafting is detrimental to the retention of the graft (Figure 5.2D).
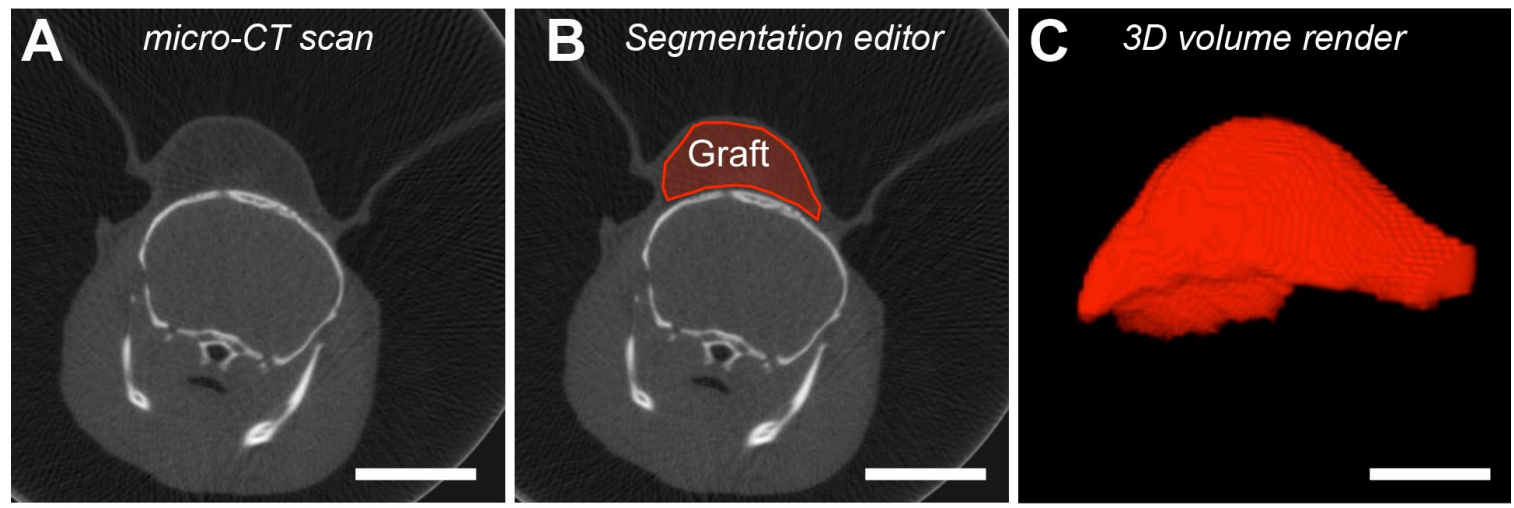

D

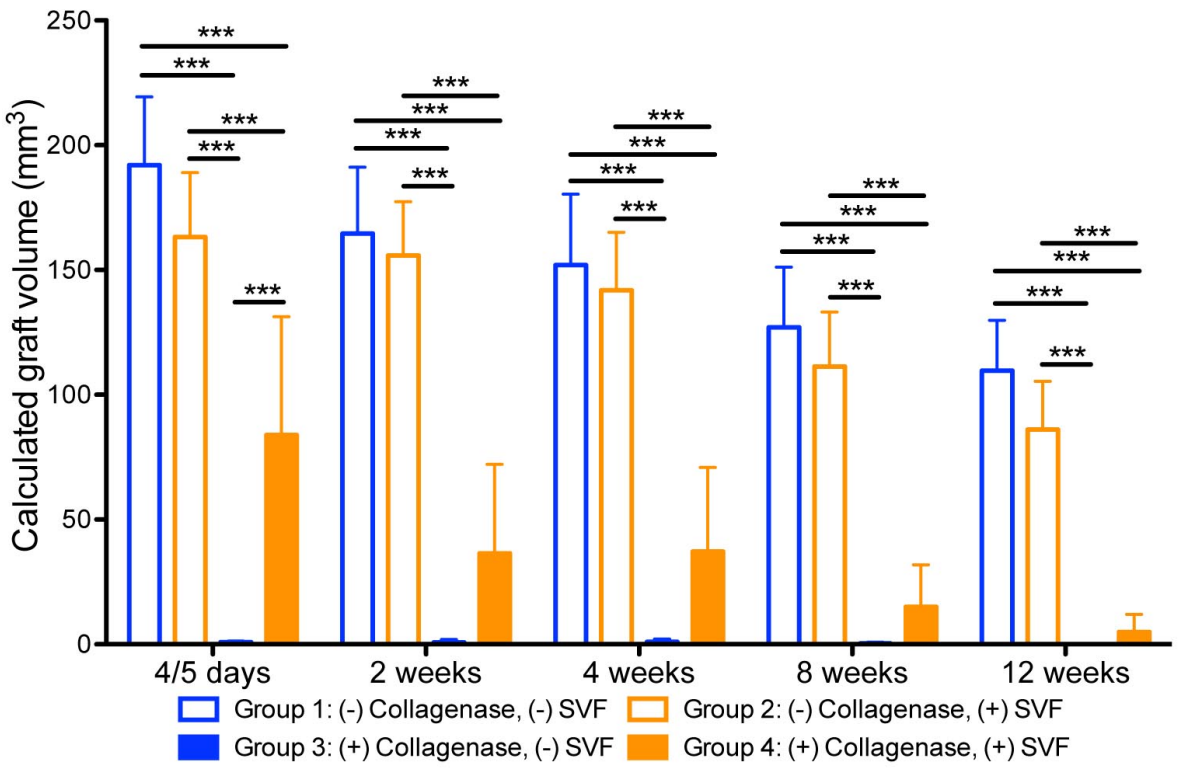

Figure 5.2: Micro-CT scanning of fat grafts

Micro-CT allows for non-invasive tracking of graft volume up to 12 weeks. A.) Example image of micro-CT scan performed on recipient mice. Scale bar $=1$ $\mathrm{cm}$. B.) Red area depicts outlined adipose graft using segmentation editor. Graft is distinguished from skin by contrast. Scale bar $=1 \mathrm{~cm}$. C.) Threedimensional volume render constructed from outlines of adipose graft. Calculation of graft volume is performed in Segmentation Editor and is reported in Panel D. Scale bar $=0.4 \mathrm{~cm}$. D.) Average calculated graft volumes for the experimental groups at time points post-implantation. Two-way ANOVA, ${ }^{* * *}=p$-value $<0.001$. All data are mean + standard deviation. $n=6$ animals for each group. 
SVF supplementation aids in volume retention of collagenase-digested grafts but does not increase volume retention of undigested grafts

As stated above, after $4 / 5$ days post-implantation the collagenase-digested grafts without SVF were nearly undetectable with an average calculated volume of 0.87 $\mathrm{mm}^{3}$, while the collagenase-digested grafts with SVF supplementation had an average calculated volume of $83.90 \mathrm{~mm}^{3}$. These data suggest that SVF supplementation of the collagenase-digested adipose grafts increased the volume retention and mitigated the resorption of the graft post-implantation. Interestingly, SVF supplementation of undigested grafts (Groups 1 and 2) did not increase the volume retention with average calculated volumes of the SVF supplemented group being slightly lower, but not statistically lower, than the unsupplemented groups at all time points (Figure 5.2D).

Collagenase digestion decrease overall tissue health as evidenced by $H \& E$ scoring metrics

The collagenase-digested grafts (Group 4) had, on average, higher scores for inflammation, cysts/vacuole presence, and fibrosis when compared to grafts that had not been collagenase digested prior to implantation (Groups 1 and 2) (Figure 5.3D). Since there were no grafts remaining at week 12 for Group 3 (collagenase digested, no SVF supplementation), H\&E scoring was not performed on this group. There was a significant increase in the amount of inflammation present, the number of cysts/vacuoles observed, and the amount of fibrosis in collagenase- 
digested when compared to undigested grafts (Figure 5.3D). There was also a striking difference in the membrane integrity of the groups - the undigested grafts (Groups 1 and 2) had much more uniform, intact adipocytes than the collagenasedigested group (Figure 5.3D). There were no statistically significant differences between Groups 1 and 2 for any metric, suggesting that SVF supplementation did not have an impact on the overall tissue health. Taken together, these data suggest, and are in agreement with volume retention outcomes, that collagenase digestion is detrimental to graft retention and overall tissue health.

\section{Terminal adipose graft masses are consistent with calculated graft volumes}

After twelve weeks, the adipose grafts were harvested and massed. The masses of the undigested groups without SVF and with SVF were 0.116 grams and 0.104, respectively (Figure 5.3E). Assuming that the initial graft was 0.225 grams (0.9 $\mathrm{g} / \mathrm{mL}$ density of adipose tissue ${ }^{265}$ ), this equated to roughly $40 \%$ volume retention rate after twelve weeks, which is consistent with the literature ${ }^{266}$. These masses were also consistent with the trends of the calculated graft volumes at twelve weeks and the $40-50 \%$ volume retention rate observed in those calculations (above). The unsupplemented grafts that were collagenase digested prior to implantation (Group 3) were undetectable at twelve weeks and not harvested (Figure 5.3E). Consistent with the calculated volumes using micro-CT scanning, the collagenase digested grafts with SVF supplementation (Group 4) had an average mass of 0.017 grams (Figure 5.3E). Taken together, these data corroborate that collagenase digestion is 
detrimental to graft survival and that SVF supplementation of digested grafts aids in volume retention.

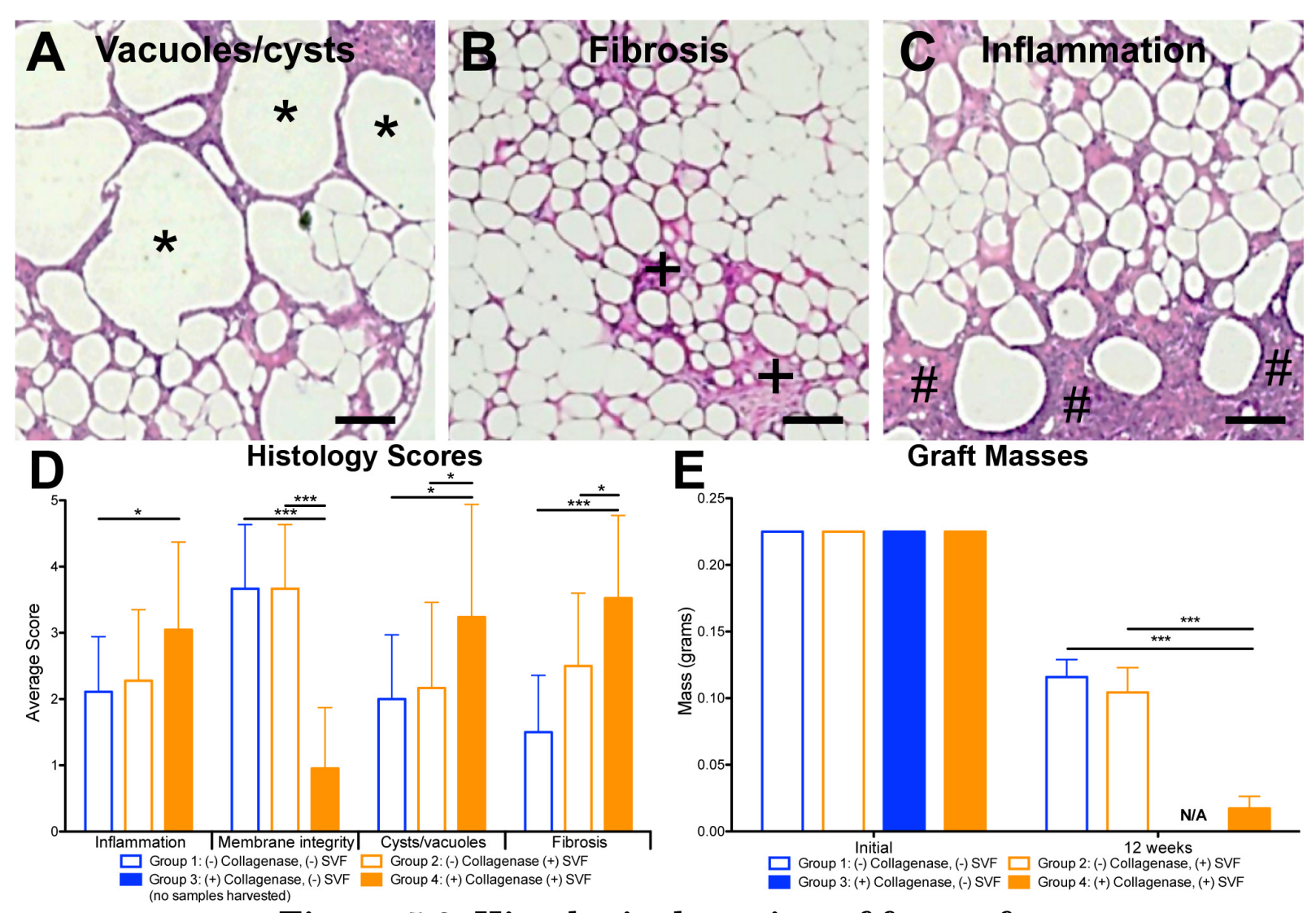

Figure 5.3: Histological scoring of fat grafts

Collagenase-digested grafts were qualitatively less healthy as compared to undigested grafts. A.) Example image of $H \& E$ stain depicting the presence of cysts and vacuoles. * denotes cyst or vacuole. B.) Example image of H\&E stain depicting the presence of fibrosis. + denotes presence of fibrosis. C.) Example image of $H \& E$ stain depicting the presence of inflammation. \# denotes presence of inflammation. $D$.) Average histological scores for each of the four metrics quantified. One-way ANOVA between each metric, ${ }^{*}=p$-value $<0.05,{ }^{* * *}=p$-value $<0.001$. E.) Average mass of grafts at 12 weeks compared to assumed mass of grafts at initial time point. Grafts were not detectable for Group 3. One-way ANOVA, *** = p-value < 0.001. All scale bars $=100 \mu \mathrm{m}$. All data are mean + standard deviation. $n=6$ animals for each group. 
Implantation of xenografts does not significantly alter body mass over the duration of the experiment

Body masses of each mouse were recorded before surgery and before each micro-CT scan was performed. As is typical for growing mice, the masses of each treatment group rose steadily from roughly 23 grams before surgery to approximately 30 grams at the terminal time point of twelve weeks (data not shown). There were no significant differences between any of the treatment groups at any of the time points measured. 


\section{Discussion}

The use of autologous fat to fill soft tissue defects has become more prevalent in reconstructive and aesthetic surgeries over the past decade ${ }^{4,228}$. Autologous fat is biocompatible, is typically readily available within a patient, easy to harvest, and allows clinicians to avoid the use of synthetic or foreign implant materials. Longterm resorption rates of autologous fat grafts range from $25-80 \%$ and are unpredictable, which has prompted clinicians and scientists to develop strategies to combat this adverse resorption process. Clinicians have started to "enhance" fat grafts by supplementing with the stromal vascular fraction that is most commonly isolated from lipoaspirate using enzymatic digestion (i.e. collagenase digestion). While other techniques can be used to isolate the SVF (mechanical, centrifugation, etc.), our current studies focused on fat grafting techniques that utilize enzymatic digestion, specifically collagenase, prior to fat grafting. Many fat grafting studies have reported the use of enzymatic digestion to isolate the SVF and subsequent supplementation of the adipocyte portion with the enzymatically-isolated $\mathrm{SVF}^{8,259,260}$. Additionally, other groups have used the collagenase-digested adipocyte portion as part of the graft without complete understanding of the effect of digestion on the adipocytes. In our previous study, we found that increasing duration of collagenase digestion decreased the viability of adipocytes and the interstitial cells within the adipose tissue. In the present study, we examined the effect of collagenase digestion on adipocytes and the impact of digestion on in vivo graft survival. We modified a published xenograft model ${ }^{141}$ and implanted human lipoaspirate as a fat graft and then serially evaluated volume retention and tissue 
health over a twelve-week time course. We found that collagenase digestion prior to grafting significantly decreased the long-term volume retention of the implanted adipose, as evidenced by non-invasive micro-CT, and decreased the overall tissue health, as determined by H\&E staining. Further, we found that SVF supplementation of collagenase-digested grafts slightly improved the volume retention, but not to the level observed using undigested grafts.

Collagenase digestion of the adipocytes prior to implantation not only detrimentally affected the long-term volume retention and overall tissue health, but micro-CT scans and volume calculations revealed rapid resorption of collagenasedigested adipose at the earliest time point ( 4 or 5 days post-implantation). The grafts from Group 3 were nearly undetectable, and the grafts from Group 4 retained only $40 \%$ of their initial volume. H\&E evaluation revealed an overall decrease in tissue health for collagenase-digested grafts (Group 3) with the adipocytes having lower membrane integrity scores (more fragmented adipocytes), higher levels of inflammation/fibrosis, and a more significant presence of cysts and vacuoles when compared to adipocytes that were not collagenase digested (Figure 5.3D). The mass of the collagenase-digested grafts was also significantly lower at the terminal time point (Figure 5.3E). Taken together, these findings suggest that collagenasedigestion prior to grafting is detrimental to the long-term volume retention of fat grafts.

Currently, it is unclear why the collagenase digestion of adipocytes prior to implantation/grafting decreases the volume retention, but previous studies may 
provide some insight. In our previous in vitro studies, for example, we observed that increasing durations of exposure to collagenase had an increasingly detrimental effect on human adipocytes and interstitial cell viability ${ }^{263}$ (Figures 4.2, 4.4). These in vitro studies suggest that in the present study, the collagenase-digested grafts had reduced viability at the onset of grafting. In another study conducted by Ruan et al., isolation of primary adipocytes from murine epididymal fat pads via collagenase digestion increased several genes that encoded several proinflammatory mediators ${ }^{267}$. Specifically, Ruan et al. reported a significant increase in TNF- $\alpha$ expression by primary adipocytes that were isolated using collagenase digestion ${ }^{267}$. Based on these findings, we speculate that collagenase digestion of the adipocytes prior to grafting induces an inflammatory environment within the graft via TNF-a secretion that leads to increased resorption of the graft. In support of this theory, Thompson et al. showed that IL-6 levels are also altered in adipocytes during collagenase digestion and that varying collagenase concentrations/rotational speeds during digestion modulates the IL-6 expression ${ }^{268}$. IL-6 and TNF- $\alpha$ have been linked to obesity-related inflammation and are generally considered proinflammatory cytokines ${ }^{269,270}$. Therefore, future studies investigating the effects of collagenase on adipose tissue retention should examine levels of these two proinflammatory cytokines, among others ${ }^{271}$, in the tissue following graft implantation.

Both pre-clinical and clinical studies have reported significant improvements in fat graft volume retention by "enhancing" or supplementing the fat grafts with SVF prior to implantation. We observed an improvement in volume retention for 
SVF-treated grafts that were collagenase-digested prior to implantation. Indeed, supplementation of collagenase-digested grafts with SVF prior to implantation (Group 4) delayed the resorption and increased the volume of the graft at each time point. Our findings are consistent with many other groups that have reported increased volume retention and delayed resorption following the supplementation of grafts with $\mathrm{SVF}^{254,259}$. It is also possible that the collagenase digestion "stripped" the adipose tissue graft of the interstitial cells and supplementing the adipose graft with SVF cells "repopulated" the interstitial cell compartment, which appears to be necessary for graft survival. We have previously reported that increasing collagenase digestion decreases the number of interstitial cells within adipose tissue 263 . This "stripping" of the interstitial cell compartment may also provide further explanation as to why collagenase digestion negatively impacts graft retention.

Interestingly, SVF supplementation of grafts that were not collagenase digested had no effect on the overall volume retention for the experimental time points. This finding differs from what has been reported in the literature, which is that SVF supplementation improves volume retention ${ }^{272}$. Our cell dosage $(12,500$ SVF cells for each $250 \mu \mathrm{L}$ fat graft) was based on a similar study conducted by Paik et al. where the concentration of SVF cells within adipose tissue was titrated to achieve an optimal effect ${ }^{254}$. Paik et al. reported that concentrations of SVF cells above a certain threshold (10 million cells $/ \mathrm{mL}$ ) decreased tissue health and caused greater lipodegeneration in a similar xenograft model. Paik et al. further reported 
that 50,000 SVF cells/mL adipose tissue was an ideal concentration, and hence this was the SVF concentration used in our study. However, this concentration is much lower than concentrations demonstrated in other studies to significantly increase volume retention. For example, Gentile et al. supplemented clinical fat grafts with $250,000 \mathrm{SVF}$ cells $/ \mathrm{mL}$ and reported less volume loss over 18 months $^{272}$. Studies which supplement grafts with ex vivo expanded ASCs have also used much higher concentrations of cells per volume of adipose tissue, with concentrations as high as $20 \times 10^{6} \mathrm{ASC} / \mathrm{mL}$ fat ${ }^{5}$. Because we did not conduct a dose-response study, we cannot draw conclusions about whether higher (or lower) doses of SVF might yield an improvement in fat graft volume retention in this murine model.

There are several other caveats to our study. First, we examined the effect of collagenase digestion on in vivo graft retention by using one digestion duration (50 minutes). This digestion duration was based on our previous study in which we found that 50 minutes was the "tipping point" beyond which adipocytes and interstitial cells started to become less viable 263 . It would be important to evaluate both shorter (30 minutes) and longer (90 minutes) collagenase digestion times to help elucidate the potential detrimental effect of collagenase digestion on in vivo graft survival. It is possible that a short collagenase digestion (less than 50 minutes) may, in fact, be beneficial to graft survival without jeopardizing the viability of the adipocytes within the graft. Short digestion durations may provide benefit to graft survival by increasing the surface area to volume ratio (smaller parcels of fat), which may promote more rapid revascularization. This theory has 
not been tested in these studies but would be interesting to consider in future. Another important consideration in our studies is the fact that all studies were conducted in immunocompromised mice (NOD SCID mice), which lack a complete immune system. While the xenograft model allows for the grafting of human adipose tissue into a mouse without rejection, the lack of a complete immune system omits a significant factor in the grafting and inflammatory process. Specifically, these mice have defective $\mathrm{T}$ and $\mathrm{B}$ cell development and have deficient natural killer (NK) cell function ${ }^{273,274}$, and it is possible that these cell types play a significant role in the resorption process. We evaluated tissue "health" by scoring several metrics (inflammation, fibrosis, membrane integrity) using $\mathrm{H} \& \mathrm{E}$ staining at twelve weeks. While these scores were subjective, they were compiled from three trained, blinded observers, and they have been used in the literature ${ }^{255,264}$ to report fat graft tissue health. Therefore, we feel confident that our scoring system provides insight into the overall tissue health of the grafts. 


\section{Conclusions}

This study extends our previous in vitro findings ${ }^{263}$, that collagenase digestion is detrimental to adipocyte and interstitial cell viability, to a clinically-relevant in vivo model. The current study suggests that collagenase digestion of adipose grafts prior to implantation adversely affects the long-term in vivo retention over the course of twelve weeks. Further, we show that supplementing collagenase-digested fat grafts with SVF enhances volume retention and delays the resorption process. Our preclinical findings help to clarify the safety and efficacy of using enzymatically digested adipocytes and SVF during autologous fat grafting. Further, this study helps to define the risks and benefits that different adipose treatments pose and may guide protocols for processing and preparing fat grafts in the clinical setting. 


\title{
Chapter 6
}

\section{PARADOXICAL ADIPOSE HYPERPLASIA AND CELLULAR EFFECTS AFTER CRYOLIPOLYSIS: A CASE REPORT}

\author{
Acknowledgements: Shruti C. Tannana, Yiqi Cao ${ }^{b}$, Shayn M. Peircea,b, \\ Thomas J. Gamppera
}

${ }^{a}$ Department of Plastic Surgery, University of Virginia

${ }^{b}$ Department of Biomedical Engineering, University of Virginia

The text included in this chapter has been published here:

Seaman SA, Tannan SC, Cao Y, Peirce SM, Gampper TJ. Paradoxical adipose hyperplasia and cellular effects after cryolipolysis: a case report. Aesthetic Surgery Journal 2016; 36(1): 6-13. 


\section{Abstract \\ Background}

Cryolipolysis is a noninvasive technique for the reduction of subcutaneous adipose tissue by controlled, localized cooling, causing adipocyte apoptosis reportedly without affecting surrounding tissue. Although cryolipolysis has a low incidence of adverse side effects, 33 cases of paradoxical adipose hyperplasia (PAH) have been reported, although the precise pathogenesis is poorly understood.

\section{Methods and results}

This present case study of a rare case of $\mathrm{PAH}$ aims to characterize the pathological changes in the adipose tissue of PAH on a cellular level by using multiple different assays to identify the underlying mechanism of PAH and reduce the prevalence of $\mathrm{PAH}$ in the future (H\&E staining, LIVE/DEAD staining, BODIPY staining). Tissue with PAH had fewer viable cells and significantly decreased quantities of interstitial cells $(\mathrm{p}=0.04)$ and fewer vessels per adipose tissue area when compared to the control tissue. Adipocytes from the PAH tissue were on average slightly smaller than the control adipocytes. Adipocytes of PAH tissue had irregularly contoured edges when compared to the smooth, round edges of the control tissue. These findings from a neutral third party are contrary to prior reports from the inventors of this technique regarding effects of cryolipolysis on both the microvasculature and interstitial cells in adipose tissue. 


\section{Conclusions}

Our use of different assays to compare cryolipolysis-treated PAH tissue with untreated adipose tissue in the same patient showed adipose tissue that developed PAH was hypocellular and hypovascular. Contrary to prior reports from the inventors, cryolipolysis may cause vessel loss, which could lead to ischemia and/or hypoxia that further contributes to adipocyte death. 


\section{Introduction}

Cryolipolysis is a noninvasive technique for the reduction of subcutaneous adipose tissue by controlled, localized cooling275,276. During this procedure, a fold of the adipose tissue is drawn between two cooling plates, and the temperature is lowered to $0^{\circ} \mathrm{C}$. Cold exposures are well-tolerated and result in fat loss without causing significant skin injury 277,278 . This procedure reportedly triggers adipocyte apoptosis without affecting surrounding tissue 275,279 . Histologic studies of porcine preclinical models show that cryolipolysis induces an inflammatory response in which adipocytes become surrounded by neutrophils, macrophages, and other phagocytic cells ${ }^{277}$. The procedure results in decreased size and disaggregation of adipocytes, which leads to a reduction in the thickness of the subcutaneous fat tissue ${ }^{277}$. Jalian et al. report that as of 2014 , there have been 650,000 cryolipolysis treatments worldwide since the introduction of the procedure ${ }^{280}$. A blinded comparison of pretreatment photos and post-cryolipolysis treatment photos of 50 subjects showed that physician reviewers were able to differentiate the two sets of photographs $92 \%$ of the time, hinting at the efficacy of cryolipolysis ${ }^{281}$. In addition, a pilot study in which six patient abdominal subcutaneous fat deposits were treated with cryolipolysis revealed that after 6 months, the clinical treatment group showed an average of $19.6 \%$ fat reduction by ultrasound imaging 279 . The American Society for Aesthetic Plastic Surgery (ASAPS) ranks nonsurgical fat reduction as the tenth most frequent nonsurgical cosmetic procedure and demonstrates its growing popularity by citing a $42.7 \%$ increase in nonsurgical fat reduction procedures from 94,922 in 2013 to 135,448 in $2014^{282}$. 
Cryolipolysis is generally considered a safe procedure with side effects ranging from edema, bruising, erythema, and transient neuralgia, and these side effects are typically self-resolving within two weeks posttreatment ${ }^{276,281}$. Stevens et al. examined medical records for over 500 patients treated with cryolipolysis and found that only three patients reported mild to moderate pain or neuralgia and found no adverse events reported ${ }^{276}$. Although cryolipolysis has a low incidence of serious adverse side effects, 33 cases of paradoxical adipose hyperplasia (PAH) (out of roughly 650,000 cryolipolysis treatments) have been reported to device manufacturers ${ }^{280}$. Derrick et al. mined the literature for articles published on cryolipolysis and found clinical reports for 1445 patients, with only two of these patients $(0.14 \%)$ reporting $\mathrm{PAH}^{283}$. In these rare cases of $\mathrm{PAH}$, the adipose tissue at the treatment site increases in mass and is clearly visible at the macroscopic scale $^{280}$. The precise pathogenesis of $\mathrm{PAH}$ is not well understood with few studies examining this phenomenon. Jalian et al. reported a case study of enlarged tissue at the treatment site and looked at this hyperplastic tissue at the microscopic and macroscopic level280. They used two modalities, magnetic resonance imaging (MRI) and hematoxylin and eosin (H\&E) staining, to study macroscopic and microscopic differences in PAH tissue as compared to untreated tissue. MRI data revealed growth of the cryolipolysis-treated tissue, while H\&E staining revealed decreased adipocyte organization, septal thickening around fat globules, and an increase in vascularity in the cryolipolysis-treated tissue. 
This present case study aims to characterize the pathological changes in the adipose tissue of PAH on a cellular level by using multiple assays (H\&E staining, LIVE/DEAD staining, BODIPY staining), an important step towards identifying the underlying mechanism of PAH and reducing the prevalence of PAH in the future. 


\section{Report of the Case}

The patient is a 48 year old female who underwent two total cryolipolysis treatments with the CoolSculpting® device (Zeltiq ${ }^{\circledR}$, Pleasonton, CA) in back-toback months. Both treatments were performed to her abdomen, posterior trunk and bilateral flanks. She had developed palpable firmness and a visible increase in the adipose tissue of her abdomen and posterior trunk three months after the second cryolipolysis treatment (Figure 6.1). These clinical findings remained unchanged six months after the second cryolipolysis treatment and were consistent with PAH. Six months after the second cryolipolysis treatment, the patient presented to our office the following month in November of 2013 and subsequently elected to undergo suction lipectomy of these hyperplastic areas. Suction lipectomy was performed 7 months after the second cryolipolysis treatment (one month after presenting to our office). At the time of the procedure, adipose tissue from both her previously treated anatomic areas with $\mathrm{PAH}$ and untreated deposits (control tissue) were sampled by direct open excision. Analysis of the tissue samples was performed by researchers blinded to the tissue source (cryolipolysis-treated tissue with subsequent PAH versus untreated, control tissue). Tissue was acquired according to an approved protocol by the Institutional Review Board at The University of Virginia. 

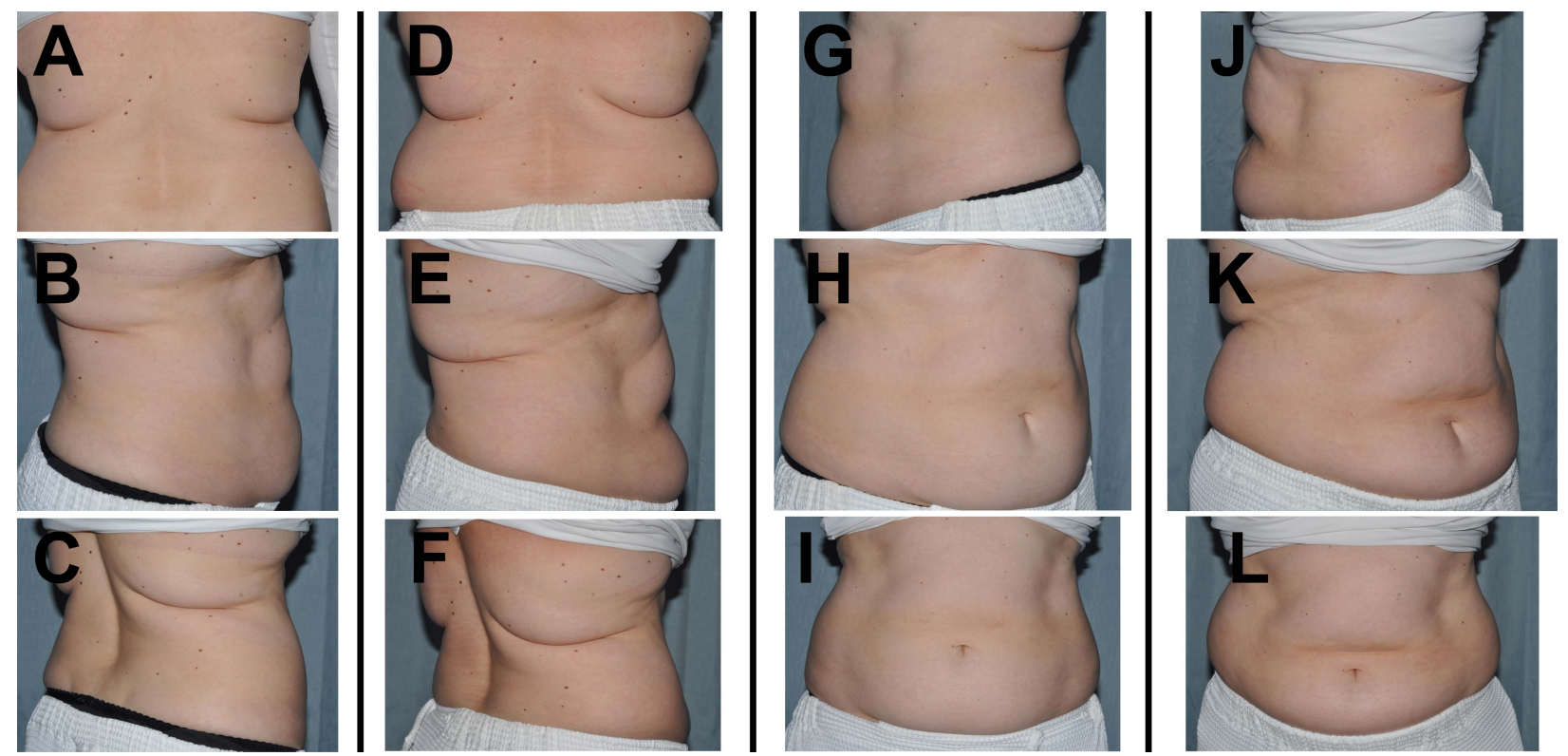

Figure 6.1: Pre-procedure and post-procedure photos of case

Post-procedure growth of adiposity is seen in the posterior brassiere-line (panels D-F) and the abdomen (panels J-L) when compared to pre-procedure photos (panels A-C, $G-I)$ of 48 year old female patient. Pre-procedure photos are shown in panels A-C and G-I. Photos taken four months after the patient's second cryolipolysis treatment are shown in panels $D-F$ and $J$-L. The entire abdomen, posterior trunk brassiere-line and flanks were treated with cryolipolysis according to manufacturer's guidelines. Photo Credit: B. Straka.

Small $\left(\sim 5 \mathrm{~mm}^{3}\right)$, excised tissue samples from control and cryolipolysis-treated areas with PAH were stained with a LIVE/DEAD kit (Life Technologies L-7013, Grand Island, NY) to assess interstitial cell viability and total number of interstitial cells (Figures 6.2A, 2B). Briefly, Components A and B were diluted 1:250 in phosphate buffered saline (PBS) and $100 \mu \mathrm{L}$ of staining solution was added to each sample tube and were incubated at room temperature for 30 minutes protected from light. Samples were washed three times with PBS and were mounted on gelatincoated slides for imaging. All samples were imaged using a Nikon TE 2000-E2 microscope equipped with a confocal attachment. Viable cells stained green and dead nonviable cells stained red, allowing for quantification of percent of viable cells 
and total number of cells. Representative images (200x magnification, $40 \mu \mathrm{m} \mathrm{Z}$ stacks) and quantification of three unique fields of view (FOV) per sample reveal a lower percentage of viable cells in the tissue with PAH when compared to the control tissue (Figure 6.2C). In addition, the total number of interstitial cells (which include vascular cells, immune cells, perivascular cells) in the tissue with PAH is significantly decreased $(\mathrm{p}=0.04)$ when compared to the control tissue (Figure 6.2D).
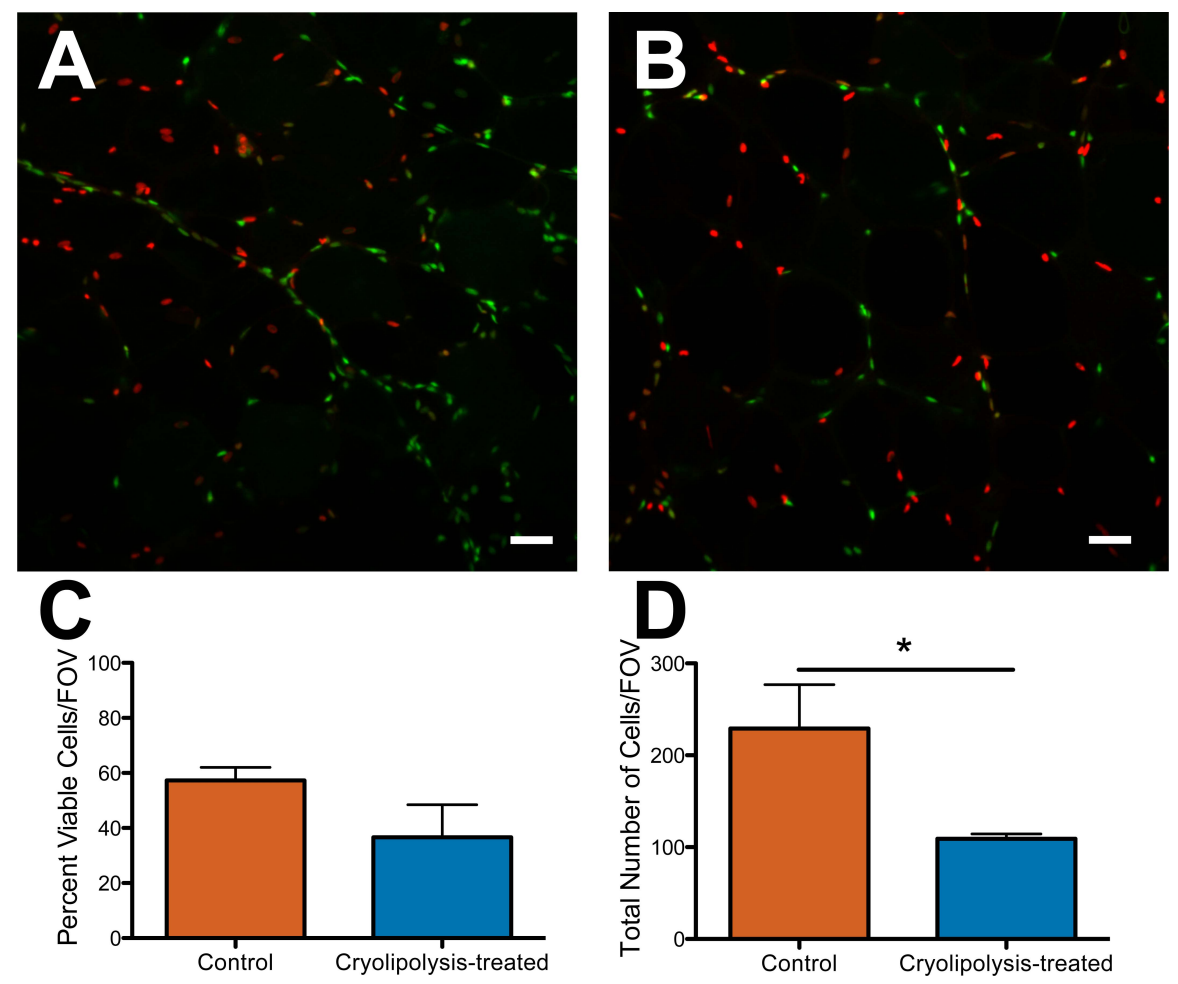

Figure 6.2: LIVE/DEAD visualization and quantification of control and cryolipolysis-treated tissue

LIVE/DEAD staining reveals a decrease in the percent of viable interstitial cells and total number of interstitial cells in cryolipolysis-treated tissue when compared to control tissue. Tissue samples from control (panel A) and cryolipolysis-treated tissue (panel B) were stained with a LIVE/DEAD kit to assess interstitial cell viability (panel $C$ ) and total number of interstitial cells (panel D). Representative images and quantification of three fields of view (FOV) reveal a lower percentage of viable cells in the cryolipolysis-treated tissue when compared to the control tissue and a statistically significant decrease in the total number of interstitial cells in the cryolipolysis-treated tissue (panel D). A two-tailed paired t-test was used to evaluate statistical significance (statistical significance asserted at p-value $<0.05$ ) for both quantification methods. Scale bar $=50 \mu \mathrm{m}$. 
Small $\left(\sim 5 \mathrm{~mm}^{3}\right)$, excised tissue samples from control and cryolipolysis-treated areas with $\mathrm{PAH}$ were fixed with $4 \%(\mathrm{v} / \mathrm{v})$ paraformaldehyde and incubated overnight at $4^{\circ} \mathrm{C}$. The number of intact adipocytes for each tissue sample was assessed using a lipid specific stain, BODIPY 558/568 (Life Technologies D-2219, Grand Island, NY), and manual counting of adipocytes that appeared intact (as defined by absence of lipid droplets and a spherical shape consistent with untreated adipose tissue). Briefly, samples were washed three times following PFA fixation and were incubated in $100 \mu \mathrm{L}$ of $10 \mu \mathrm{g} / \mathrm{mL}$ BODIPY/PBS solution for 20 minutes at $37^{\circ} \mathrm{C}$ protected from light. Adipose tissue samples were also stained concurrently with isolectin GS-IB 4 AlexaFluor ${ }^{\circledR} 647$ (1:200 dilution, Life Technologies I32450, Grand Island, NY), which binds to endothelial cells, to visualize the extent of vascularization in the adipose tissue (Figure 6.3). The isolectin stain was incubated overnight at $4^{\circ} \mathrm{C}$ protected from light. Samples were washed three times with PBS and were mounted on gelatin-coated slides for imaging. Confocal microscopy was used to acquire 200x magnification, $40 \mu \mathrm{m}$ thick Z-stacks of four unique FOVs per sample. Representative images (Figure 6.3A, 6.3B) and quantification of these images (Figure 6.3C) via manual counting as described above of intact adipocytes revealed similar numbers of intact adipocytes per field of view in adipose with PAH and untreated adipose. It was qualitatively apparent in these images that the tissue with PAH had fewer blood vessels (less isolectin staining) than the control tissue (Figures 6.3A, 6.3B). 

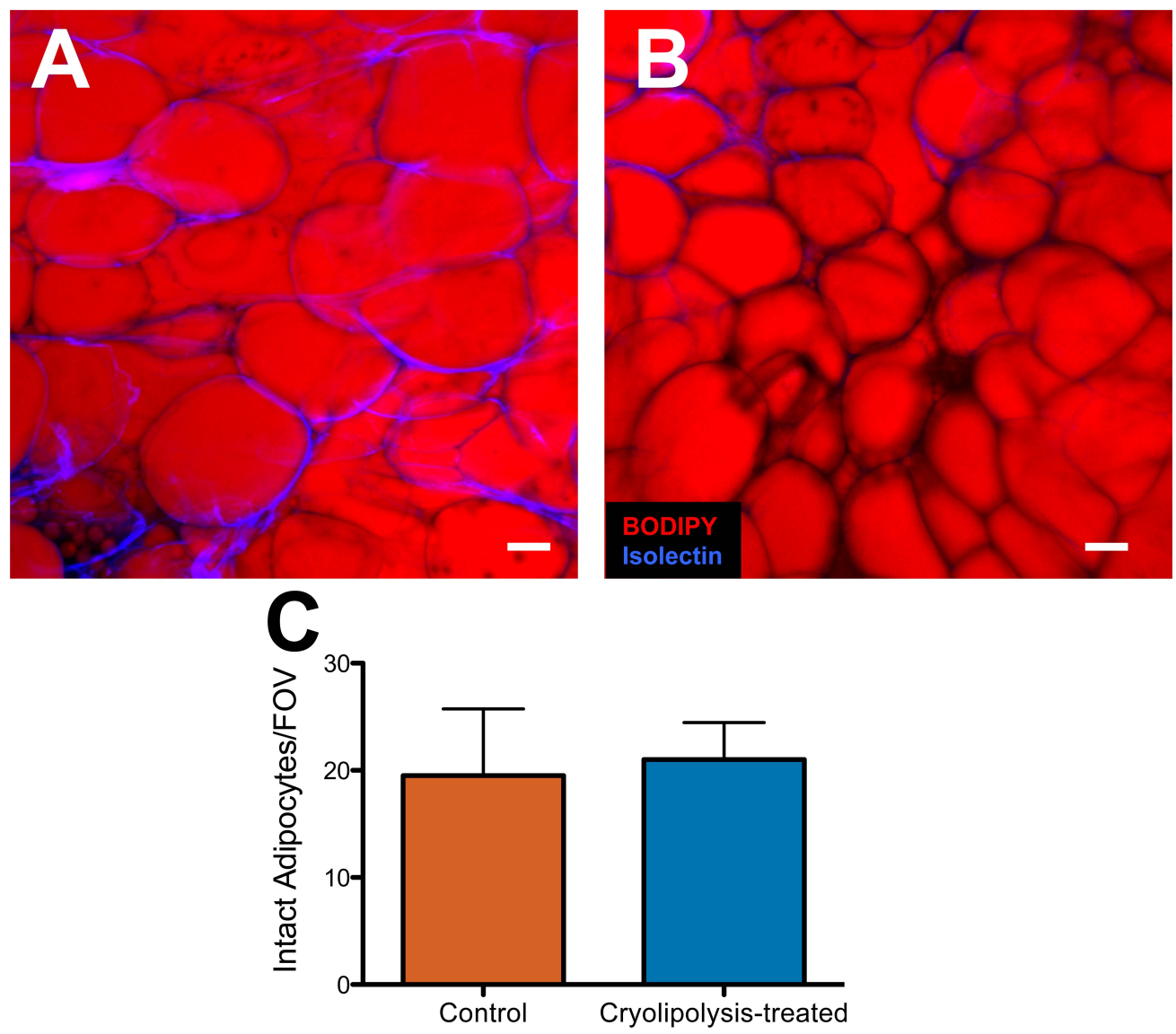

Figure 6.3: Adiposity and vascularity of control and cryolipolysis-treated tissue

BODIPY staining shows no difference in the number of intact adipocytes between groups but isolectin staining shows increased vessel staining in control tissue (panel A) when compared to cryolipolysis-treated tissue (panel B). Representative images and quantification reveal no statistical difference (two-tailed paired t-test, statistical significance asserted at p-value $<0.05)$ between the number of intact adipocytes per field of view in control adipose tissue and cryolipolysis-treated adipose tissue (panel $C$ ). Images reveal that the cryolipolysis-treated tissue (panel B) has a much lower vessel density (less isolectin staining) than the control tissue (panel A). Scale bar = $50 \mathrm{um}$.

Additional control and cryolipolysis-treated samples were processed for $\mathrm{H} \& \mathrm{E}$ staining to visualize excised fat in cross section in order to quantify vessel density and adipocyte area (Figure 6.4). Multiple images were acquired at 100x magnification using a Nikon TE 2000-E2 microscope and were montaged together to provide a complete image of the tissue slice. The entire area of the montage 
analyzed of the control tissue was $14.00 \mathrm{~mm}^{2}$ and the cryolipolysis-treated was 4.87 $\mathrm{mm}^{2}$ (data not shown). Arrows in Figure 6.4 indicate examples of vessels that were counted. The number of vessels per adipose tissue per montage area was increased in the control tissue (1.143 vessels $/ \mathrm{mm}^{2}$ adipose tissue) when compared to the tissue with PAH (0.616 vessels $/ \mathrm{mm}^{2}$ adipose tissue), consistent with observations of the BODIPY and isolectin stained tissues (Figure 6.3). The cross-sectional areas of twenty randomly selected adipocytes were measured, and the adipocytes from the $\mathrm{PAH}$ tissue were on average slightly smaller than the control adipocytes (Figure 6.4E). The average number of adipocytes per area was also quantified and there was no significant difference between the number of adipocytes per area (Figure 6.4F). Interestingly, individual adipocytes in the tissue with $\mathrm{PAH}$ appear to have irregularly contoured edges (Figure 6.4B, 6.4D) when compared to the smooth, round edges of the control tissue (Figure 6.4A, 6.4C). These irregular surfaces (possible plasma membrane blebbing) may be the preceding step to necrosis or apoptosis $^{284}$ and would be consistent with the mechanism of cryolipolysis treatment - the induction of apoptosis. 

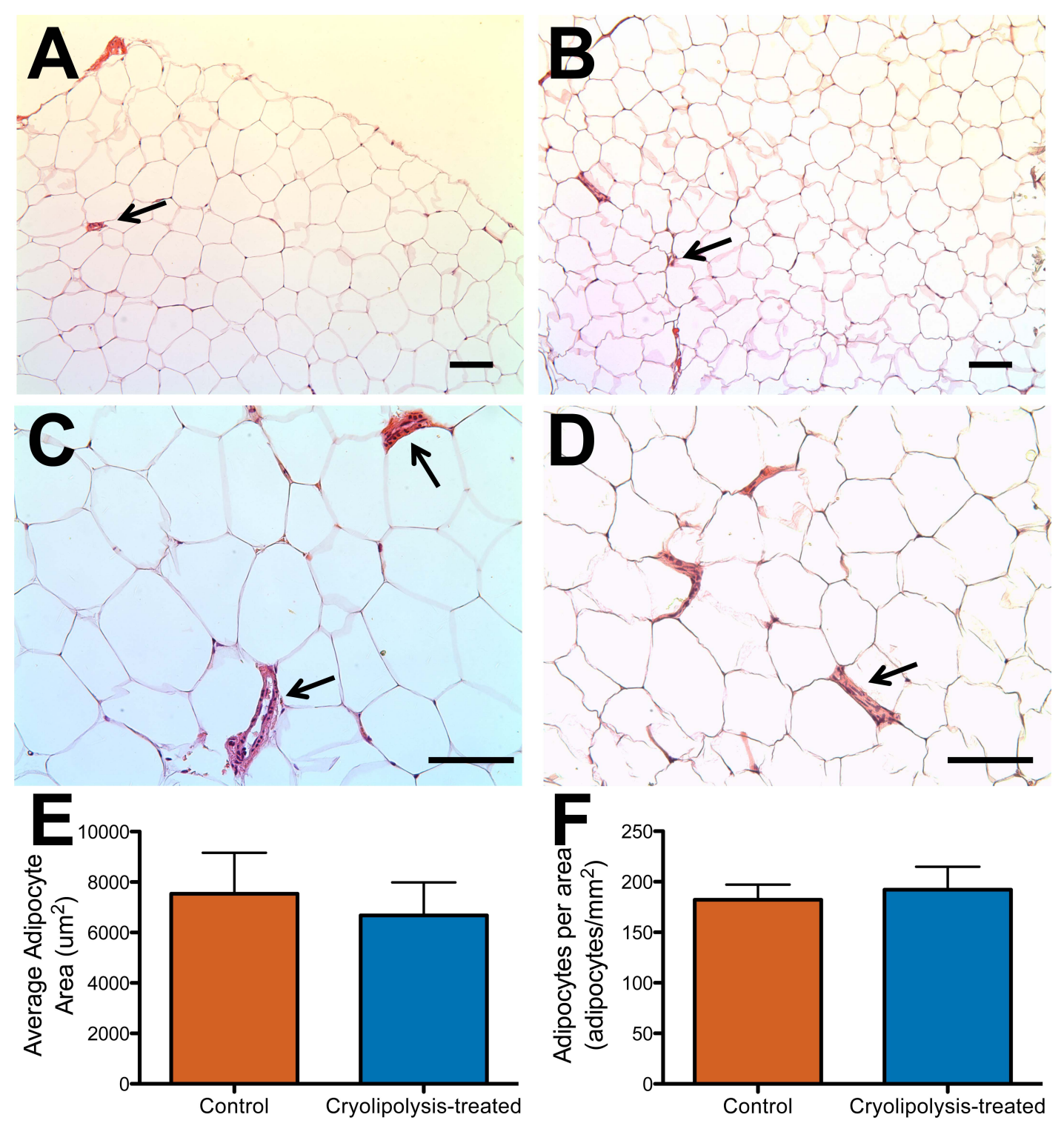

Figure 6.4: Morphological assessment of control and cryolipolysis-treated tissue

Hematoxylin and eosin staining reveals no significant difference in average adipocyte area or adipocytes per area. Vessels were counted (arrows) and the number of vessels per adipose tissue area was increased in the control tissue (panels $A, C$ ) when compared to the cryolipolysis-treated tissue (panels B,D) (data not shown). Cryolipolysis-treated tissue adipocytes (panels B,D) were on average slightly smaller than the control tissue adipocytes (panel E) with similar number of adipocytes per area in both control and cryolipolysis-treated tissues (panel F). All statistical tests: two-tailed paired $t$-test, statistical significance asserted at $p$-value $<0.05$. Scale bar $=$ $50 \mathrm{Hm}$. 


\section{Discussion}

Cryolipolysis is being increasingly commercialized in both plastic surgery and nonsurgical subspecialties as a noninvasive alternative to surgical treatment of excess adipose tissue $276-278,283$. The technique was initially approved in 2008 by the United States Food and Drug Administration for treatment of focal adiposity of the flanks, and then expanded to abdominal lipodystrophy in 2011. This innovation represents the marriage of a thoughtful observation - the recognition of fat atrophy from "popsicle panniculitis," an often-treated entity by dermatologists - with the translation of that clinical observation into a treatment modality.

The published mechanism of cryolipolysis is selective cell death of adipocytes following cold exposure. The adipocytes crystallize, undergo apoptosis, and are then eliminated by macrophage engulfment after approximately 3 months ${ }^{285}$. The inflammatory process is thought to peak at $2-4$ weeks and be complete by the 3 month mark ${ }^{286}$. CoolSculpting ${ }^{\circledR}$ treatments are reportedly specific to adipocytes and per the inventors and device manufacturers do not affect surrounding cells or tissues, but the mechanisms of cell death and eventual elimination are not well understood ${ }^{281}$. There is a rare adverse event after cryolipolysis, PAH, and the mechanism behind this process is not well understood ${ }^{280}$. Jalian et al. report a case of PAH and found decreased adipocyte organization and an increase in vascularity of $\mathrm{PAH}$ tissue when compared to control tissue after observation using $\mathrm{H} \& \mathrm{E}$ staining and MRI.

Interestingly, our findings are contrary to prior reports from the inventors of this technique regarding both the vascular changes associated with $\mathrm{PAH}$, as well as 


\section{CHAPTER 6: PARADOXICAL ADIPOSE HYPERPLASIA}

the deleterious effects of cryolipolysis on interstitial cells in adipose tissue ${ }^{5,6,8,9}$. Our use of different assays to compare cryolipolysis-treated PAH tissue with untreated adipose tissue in the same patient showed that the adipose tissue that developed PAH was both hypocellular and hypovascular. Specifically, our results of the LIVE/DEAD kit imaging reveals a decrease in the number of viable interstitial cells in tissue with $\mathrm{PAH}$ following cryolipolysis treatment. These interstitial cells include macrophages, endothelial cells, adipose derived stem cells, blood-derived cells and constitute a complex niche within the adipose tissue that plays a role in adipose tissue remodeling ${ }^{106}$. To confirm these findings, an endothelial cell-specific stain and high magnification quantification of $\mathrm{H} \& \mathrm{E}$ stain was performed to determine the qualitative vascularity in the different adipose tissue depots and found that the cryolipolysis-treated tissue with PAH was less vascularized than control tissue. Our studies do not reveal significant changes in adipocyte areas, adipocytes per area, or the number of intact adipocytes.

Although the exact mechanisms for adipocyte death and elimination following cold exposure are not known, previous studies suggest that cold exposure exclusively triggers adipocytes apoptosis ${ }^{281}$. Our study further suggests that in PAH cases, the low temperatures may have deleterious effects on the vascular cells in the interstitium of adipose tissue and may cause complications during adipose tissue recovery. Hence, it is possible that in addition to having direct effects on adipocytes, cryolipolysis may also cause vessel loss, which could lead to ischemia and/or hypoxia that further contributes to adipocyte death. 
Although $\mathrm{PAH}$ is characterized by the macroscopic appearance of adipose tissue enlargement upon clinical presentation, our data suggest that the microstructure of PAH tissue may be hypovascular and hypocellular. It is counterintuitive that a decrease in vascularity and interstitial cells could be commensurate with clinically enlarged adipose depots in PAH tissue after cryolipolysis. One would expect that an enlarged adipose depot would be accompanied or enabled by increased vessel density because a larger, more hyperplastic adipose tissue depot would require additional delivery of nutrients and oxygen.

Though it is believed that PAH clinically presents itself with enlarged mass of adipose tissue, it is difficult to measure the actual mass of the cryolipolysistreated area for our group and others ${ }^{280}$ before and after the procedure. It is possible that the affected area is not actually enlarging in mass, but rather staying the same and is merely a relative increase in adipose tissue. It may also be possible that there is a decrease in adipose tissue at the periphery of the cryolipolysis applicator, which causes a perceived increase in adipose tissue in the central areas of the cryolipolysis applicator consistent with the symptoms of PAH. MRI images helped visualize the PAH region in the study by Jalian et al. ${ }^{280}$, but both our study and theirs have not been able to confirm this perceived increase in mass. A case report of $\mathrm{PAH}$ presented by Stefani postulates that the negative suction process of the cryolipolysis instrument may stimulate adipocyte proliferation (similar to the effect of the BRAVA system on the breast), which leads to a paradoxical increase in adipose 
tissue 287 . In our study, the patient presented with palpable stiffening of the affected adipose tissue in conjunction along with a perceived increase in adipose mass. Although it is unclear what is the causing this stiffening, we surmise that it may be due to fibrosis due to the less vascularized, more hypoxic affected adipose tissue. In response to hypoxia/ischemia, resident fibroblasts rearrange the extracellular matrix and produce collagen, two hallmarks of fibrosis, in adipose tissue ${ }^{288}$, renal tissue $^{289}$, and cardiac tissue ${ }^{290}$. It is possible that this potential fibrosis caused the adipocytes within the affected PAH area to take on irregular formation (membrane blebbing, abnormal edges) in response to the changing extracellular matrix. It is also possible that the reported increased mass of PAH may be due to this fibrosis of the adipose tissue in which the extracellular matrix is expanding. The notion that fibrosis is the cause of observed PAH is speculative at this point and further studies are needed to characterize if this $\mathrm{PAH}$ phenomenon may be attributed to fibrosis of the adipose tissue.

Our studies analyzed the PAH tissue at a later timepoint than the Jalian et al. report (7 months vs. 3 months post-treatment) and it is possible that these two studies observed different stages on the spectrum of PAH. Since adipose tissue is a dynamic microenvironment with the capability to expand and decrease in size, this process is best understood as a continuum of changing cellular events. Jalian et al. may have reported the early stages of PAH (aggressive adipose tissue hyperplasia, increased angiogenesis/vessel ingrowth) and our studies may have captured the later stages (adipocyte apoptosis or homeostasis, reduction of vessel ingrowth and 
angiogenesis, ceasing of hyperplastic process). This differing time of examination may explain the discrepancy in vascularity between the two studies. The effects cryolipolysis and its induction of PAH may not even be fully captured in the assays performed in our study or the Jalian et al. study. Although our studies have focused on vascularity and adipocyte changes, there may be other cells (i.e. macrophages, tissue-resident stem cells, and fibroblasts) that have key roles in the PAH phenomenon that have yet to be examined. 


\section{Conclusions}

Additional studies are needed to better understand the mechanism of cryolipolysis and how it affects the interstitial cells and vasculature. The current study suggests that all cells (interstitial cells and adipose cells) are be affected by the treatment. As reported previously by Stefani, when PAH is suspected after multiple cryolipolysis treatments, cryolipolysis therapy should be discontinued and surgical treatment (liposuction) of the hyperplastic deposits is recommended ${ }^{287}$. Further study is needed characterize who is more at risk of developing an adverse event, and how they can be identified pre-procedure. 


\section{Chapter 7}

\section{USING DUAL-EMISSIVE OXYGEN- SENSITIVE PLA NANOPARTICLES TO MONITOR LOCAL WOUND OXYGENATION}

Acknowledgements: Christopher A. DeRosaa, Alexander S. Mathewa, Catherine M. Gorick ${ }^{b}$, Ziyi Fana , James N. Demas ${ }^{a}$, Shayn M. Peirceb, Cassandra L. Frasera,b

${ }^{a}$ Department of Chemistry, University of Virginia

${ }^{b}$ Department of Biomedical Engineering, University of Virginia 


\section{Abstract}

Objective

Optical oxygen sensing has become a premier analytical technique for oxygen quantification and could be extremely useful in a clinical setting for monitoring wounds, burns, and transferred tissue flaps. Phosphorescent materials with high $\mathrm{O}_{2}$ sensitivity and good photostability are required to reliably report oxygen levels. Dual-emissive difluoroboron $\beta$-diketonate-polylactides have both short-lived fluorescence, and long-lived, oxygen sensitive phosphorescence. The objective of this study was to validate a novel compound for sensing wound oxygenation using a murine full-thickness cutaneous wound model.

\section{Methods and Results}

We first optimized a halide (iodide) substituted naphthyl-phenyl difluoroboron $\beta$-diketone, conjugated to poly(lactic acid) $\left(\mathrm{BF}_{2} \mathrm{nbm}(\mathrm{I}) \mathrm{PLA}\right)$, which exhibits distinct fluorescence $(450 \mathrm{~nm})$ and phosphorescence $(565 \mathrm{~nm})$ emission spectra, as well as intense room temperature phosphorescence. We made 3 - $\mathrm{mm}$ diameter excisional full-thickness wounds on the dorsum of 8-week old male mice and topically delivered the compound to the wound in the form of nanoparticles. At different time points following the initial wounding, we used a high-speed, color camera for RGB ratiometry and oxygen quantification. We observed that the nanoparticles were able to spatially resolve local wound oxygenation levels within the wound bed throughout the course of healing ( $\sim 5$ days). 


\section{Conclusions}

In this study, we demonstrate the unprecedented versatility of halide substituted difluoroboron $\beta$-diketonate in the form of biodegradable nanoparticles, and their usefulness in monitoring wound oxygenation levels throughout the duration of healing in a murine full thickness skin wound model imaged with an off-the-shelf camera. We show the ability of the difluoroboron $\beta$-diketonates nanoparticles to spatially resolve local oxygenation levels within a murine wound bed, which is a first step toward their ultimate use as part of a clinical oxygen-sensing device. 


\section{Introduction}

The annual incidence of chronic wounds, including pressure, venous and diabetic ulcers, is estimated to be six million patients with estimated costs to manage these wounds exceeding $\$ 20$ billion per year ${ }^{291}$. Partial flap necrosis and ultimate failure is still a significant problem and failure rates have been reported to be as high as $31 \%$ in breast and head/neck reconstructive surgeries ${ }^{292-294}$. Plastic surgeons are often forced to determine if an open/chronic wound or burn is progressing towards healing or whether a recently transplanted tissue flap will survive after surgery. Proper vascularization and subsequent oxygenation of the tissue within the wound/burn/flap is thought to be the crux of this healing response. The oxygen level in a wound/burn or flap is a powerful indicator of healing potential, yet the current clinical methods for assessing oxygenation within these tissues are expensive, provide only point measurements, and rely on indirect measurements to quantify oxygenation (e.g. hemoglobin saturation) ${ }^{295,296}$. Clinical wound and burn assessment of healing is largely qualitative and can be highly subjective depending on which techniques are employed. The most commonly used metric to assess wound/burn healing is wound size (length and width), but shrinkage in wound size has only loosely been shown to correlate with wound healing. Plastic surgeons and nurses are required to qualitatively monitor the open wound/burn or flap after surgery on an hourly basis to try and determine the open wound/burn healing potential and the chance for flap survival.

The tools currently available for measuring tissue oxygenation levels (transcutaneous oxygen tension, TcPO2) involve the use of electrodes that are either 
temporarily adhered to the wound border or flap (cutaneously) or inserted invasively (subcutaneously) directly into the wound bed or flap ${ }^{297}$. Both of these electrode-based approaches provide tissue oxygenation read-outs at single point locations in and around the wound, depending on where they are positioned. Cutaneous electrodes that are adhered to the skin surrounding the wound elevate the temperature of the skin, which contributes substantial artifacts to the oxygen reading. Near infrared spectroscopy (NIRS) or hyperspectral spectroscopy are, on rare occasions, used clinically to provide a subsurface mapping (down to approximately 3 millimeters in depth) of oxygenated vs. deoxygenated hemoglobin levels across the area of the wound ${ }^{298}$. However, hemoglobin oxygenation levels only indicate the level of oxygen in the blood that perfused the wound bed, and this metric is not necessarily indicative of the amount of oxygen available for metabolism and healing by tissue-resident cells. The clinical need for an inexpensive, accurate, quantitative diagnostic tool that is capable of detecting an actual tissue oxygen level across the entire wound area or tissue flap is readily apparent.

Phosphorescent probes are logical alternative to hyperspectral spectroscopy or electrode diagnosis tool, as the optical properties are directly correlated with the amount of oxygen in the environment ${ }^{299,300}$. Commonly used phosphors are organometallic complexes, such as palladium and platinum metalloporphyrins ${ }^{301-}$ ${ }^{303}$, cyclometalated iridium dyes ${ }^{304}$, or ruthenium bipyridines ${ }^{305}$. These complexes exhibit only phosphorescence (P), therefore individually, these phosphors quantify 
oxygen via emission intensity or lifetime decay. Intensity based measurements are unreliable, considering the high dependence on phosphor concentration, and phosphorescence lifetimes of these metal complexes are on the magnitude of several nanoseconds to microseconds, which requires specialized equipment for detecting. When oxygen insensitive fluorophores ${ }^{305,306}$, fluorescent polymers ${ }^{307-309}$, or quantum $\operatorname{dots}^{302,310}$, are used in combination with metal phosphors, ratiometric imaging can be performed. The fluorescence (F) acts as an internal standard to monitor phosphor concentration, while the phosphorescence responds to oxygen. The resultant phosphor/fluorophore/polymer "cocktails" can be used as films or fabricated as nanoprobes. While great advances have been achieved with the described materials, it is our goal to use metal-free, biodegradable materials for biological oxygen sensing, to reduce cost, toxicity and material complexity.

In recent years, boron $\beta$-diketonates have become a versatile group of chromophores. Materials have been prepared as mechanofluorochromic powders ${ }^{311,312}$, solution-processible memory devices ${ }^{313}$, triplet emitters ${ }^{314}$, twophoton absorbers ${ }^{315}$, and aggregation-induced b-amyloid turn-on probes ${ }^{316}$. When difluoroboron is coordinated to the $\beta$-diketone core and confined to a rigid matrix, such as poly(lactic acid) (PLA), dual emission is achieved ${ }^{317-319}$. These materials exhibit both fluorescence (F), and long-lived, room temperature phosphorescence (P) in the solid state. The $\mathrm{F}$ acts as an internal standard, insensitive to oxygen, as the $\mathrm{P}$ changes intensity based on the oxygen level in the environment. By comparing the $\mathrm{F}$ to $\mathrm{P}$ (F/P ratio), the oxygen levels have been quantified by ratiometry. With PLA 
as the medium, preparation of films ${ }^{320}$, nanofibers ${ }^{321}$, or nanoparticles ${ }^{322}$, can be achieved through the various fabrication methods. In addition, this material is a metal-free, biocompatiable, and single-component, reducing both cost and nanoprobe complexity.

Here, we perform proof-of-concept studies in the development of wound diagnostic device by using this dual-emissive dye in nanoparticle form and applying these nanoparticles to a murine full thickness skin wound model to measure local wound oxygenation. We developed a portable, cost-effective high-speed color camera for this material that is capable of gathering the long-lived, phosphorescence lifetime (up to $2 \mathrm{msec}$ resolution), and red/blue/green (RGB) referenced intensity mapping for oxygen quantification ${ }^{323,324}$. The fluorescence fits within the blue channel, and the phosphorescence coincides with the red channel. The camera imaging system, in combination with this versatile set of dual-emitting dyes in nanoparticle form, can become powerful tools for monitoring molecular $\mathrm{O}_{2}$ in biological environments. The proof-of-concept in vivo studies presented in this chapter show the usefulness of these dual-emissive nanoparticles in monitoring local wound oxygenation. 


\section{Materials and Methods}

Dual-emissive iodide polymer fabrication

The iodide substituted polymer was prepared as previously described ${ }^{325}$ except the initiator $\mathrm{BF}_{2} \mathrm{nbm}(\mathrm{I}) \mathrm{OH}$ was used in place of $\mathrm{BF}_{2} \mathrm{nbm}(\mathrm{Br}) \mathrm{OH}$ (loading = initiator : lactide : catalyst; $1: 200: 0.025$ ), and stirred at $130^{\circ} \mathrm{C}$ for $5 \mathrm{~h}$, to yield a yellow crystalline powder; $345 \mathrm{mg}$ ( $51 \%$ yield, corrected for $65 \%$ polymer conversion): $\mathrm{M}_{\mathrm{n}}(\mathrm{GPC} / \mathrm{MALS})=16300 \mathrm{Da}, \mathrm{Ð}=1.17 ; \mathrm{M}_{\mathrm{w}}\left({ }^{1} \mathrm{H} \mathrm{NMR}\right)=19300 \mathrm{Da} \cdot{ }^{1} \mathrm{H}$ NMR $(600$ $\left.\mathrm{MHz}_{\mathrm{CDCl}}\right): \delta 8.71\left(\mathrm{~s}, 1 \mathrm{H}, 1^{\prime}-\mathrm{ArH}\right), 8.33\left(\mathrm{~s}, 1 \mathrm{H}, 5^{\prime}-\mathrm{ArH}\right), 8.20\left(\mathrm{~d}, \mathrm{~J}=6,2 \mathrm{H}, 2^{\prime \prime}, 6^{\prime \prime}\right.$ - $\operatorname{ArH}), 8.09\left(\mathrm{~d}, \mathrm{~J}=6,1 \mathrm{H}, 8^{\prime}-\operatorname{ArH}\right), 7.85$ (m, broad, 2H, 3', 4' - ArH) $7.73(\mathrm{~d}, \mathrm{~J}=12$, 1H, 7' - ArH), $7.16(\mathrm{~s}, 1 \mathrm{H}, \mathrm{COCHCO}), 7.04\left(\mathrm{~d}, \mathrm{~J}=12,2 \mathrm{H}, 3^{\prime \prime}, 5^{\prime \prime}-\mathrm{ArH}\right), 5.23-5.12(\mathrm{~m}$, broad, 268H, PLA- $H$ ), 4.55 (s, broad, $\left.2 \mathrm{H}, \mathrm{Ar}-\mathrm{OCH}_{2} \mathrm{CH}_{2} \mathrm{OH}\right), 4.32$ (m, broad, $2 \mathrm{H}, \mathrm{Ar}-$ $\mathrm{OCH}_{2} \mathrm{CH}_{2} \mathrm{OH}$ ), 1.58-1.53 (m, broad, $1137 \mathrm{H}, \mathrm{PLA}-\mathrm{CH}{ }_{3}$.

\section{Nanoparticle fabrication}

Nanoparticles of the iodide polymer material (termed: BNPs) were prepared as previously described by $\mathrm{DMF} / \mathrm{H}_{2} \mathrm{O}$ precipitation into deionized water at $\sim 1 \mathrm{mg} / \mathrm{mL}$ concentration $^{322}$. Cellular isotonic conditions were achieved by the addition of dextrose to achieve a $5 \%$ dextrose $/ \mathrm{NP} / \mathrm{H}_{2} \mathrm{O}$ solution. The $\mathrm{BNP}$ solution $(\sim 6 \mathrm{~mL}$ of $\sim 1$ $\mathrm{mg} / \mathrm{mL}$ ) was concentrated by centrifugation at $4000 \mathrm{RPM}$ at room temperature for three minutes (Sorval, ThermoScientific, Legend RT) in a concentrator centrifuge tube (Amicon Ultra, Regenerated Celluose, 30,000 MW cutoff) to yield $\sim 3 \mathrm{~mL}$ of $\sim 2$ $\mathrm{mg} / \mathrm{mL}$ BNP solution. The $1 \mathrm{~mL}$ of $\mathrm{BNP}(\sim 2 \mathrm{mg} / \mathrm{mL})$ solution was filtered through a $200 \mathrm{~nm}$ filter (Whatman) before being combined with $1 \mathrm{~mL}$ of $10 \%$ dextrose solution 
to yield $2 \mathrm{~mL}$ of $\sim 1 \mathrm{mg} / \mathrm{mL}$ BNP solution at $5 \%$ dextrose concentration. BNP solutions were stored at $4^{\circ} \mathrm{C}$ prior to use, and filtered (200 $\mathrm{nm}$ filter, Whatman) before application for sterilization purposes.

\section{Characterization of material luminescence}

The fluorescence emission spectra of the material were recorded on a Horiba Fluorolog-3 Model FL3-22 spectrofluorometer (double-grating excitation and doublegrating emission monochromators). The phosphorescence emission spectra were recorded with the same instrument as above but a pulsed xenon lamp (369 $\mathrm{nm}$ excitation, duration $<1 \mathrm{msec}$ ) was used and the phosphorescence spectra were collected with a one msec delay after exciting the sample. Thin films of the material were prepared on the inner wall of glass vials by dissolving the iodide polymer in $\mathrm{CH}_{2} \mathrm{Cl}_{2}(2 \mathrm{mg} / \mathrm{mL})$ and evaporating the solvent by slowly rotating the vial under a low stream of nitrogen. These solution-cast films were dried in vacuo for at least fifteen minutes before measurements (fluorescence and phosphorescence) were taken. Fluorescence spectra and lifetimes were obtained under ambient conditions (e.g., air, 21\% oxygen), while phosphorescence measurements were obtained under a purged, $\mathrm{N}_{2}$ atmosphere ( $0 \%$ oxygen) (Figure $\left.7.1 \mathrm{~A}\right)$. The vials with the solutioncast, iodide polymer films were purged of oxygen and sealed with a Teflon cap and then wrapped in Parafilm in a glove box prior to measurements. The glove box was purged for thirty minutes prior to samples being sealed. 
Oxygen sensitivity analysis of the BNPs was performed as described previously ${ }^{320}$ by making various gas mixtures of oxygen and nitrogen to make concentrations of oxygen from $0-21 \%$. Briefly, BNPs in glass vials were fitted with a 12 millimeter PTFE/silicone/PTFE seal (Chromatography Research Supplies), connected by a septum screw cap. For $21 \%$ oxygen (i.e. air), measurements were taken under ambient conditions (open vial, no cap). For 0\% $\mathrm{O}_{2}$ (analytical grade nitrogen, Praxair) was purged through the vial for fifteen minutes (Figure 7.1B). For oxygen concentrations between $0 \%$ and $21 \%$, a Cole Parmer $65 \mathrm{~mm}$ flowmeter was used to introduce varying oxygen concentrations to the spin-cast films through a septum screw cap. Ratiometric (blue to red channel) intensities were calculated for the various oxygen concentrations to construct an oxygen standard curve (Figure 7.1C). This oxygen standard curve was used to calculate approximate oxygen concentrations in future in vivo studies.
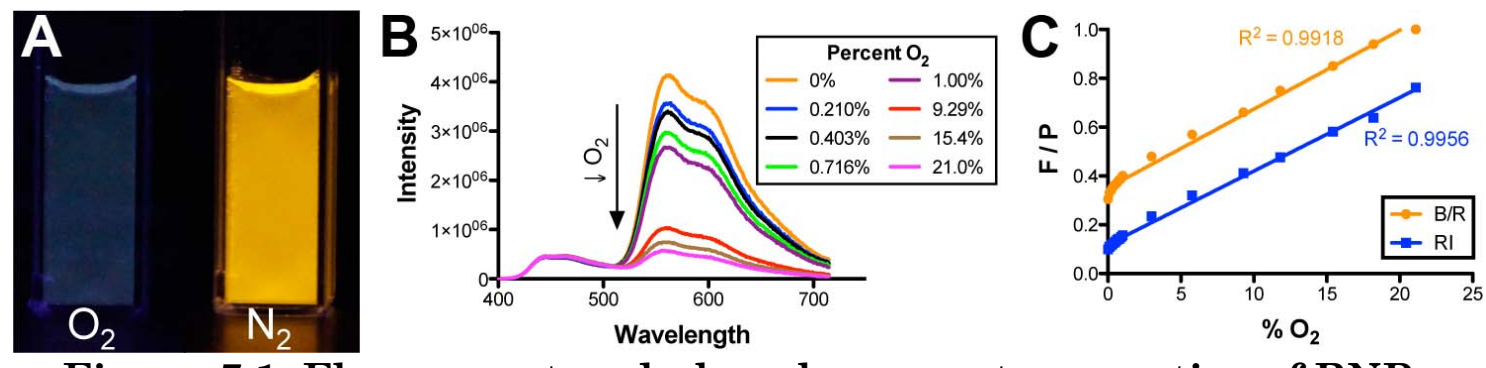

Figure 7.1: Fluorescent and phosphorescent properties of BNPs BNPs exhibit distinct fluorescent and phosphorescent emissions and display intense room temperature, oxygen-sensitive phosphorescence. A.) Images of oxygen $\left(\mathrm{O}_{2}\right)$ and nitrogen $\left(\mathrm{N}_{2}\right)$ purged nanoparticles depicted intense room temperature phosphorescence of BNPs. B.) Total emission spectra of BNPs at various oxygen levels. C.) Ratiometric calibration of BNPs between 0-21\% [O $\mathrm{O}_{2}$. $R I=$ reference intensity using fluorimeter, $B / R=$ blue channel $/$ red channel $R G B$ calibration performed by handheld camera. 


\section{Murine full thickness skin wound model}

All procedures were performed in accordance with the University of Virginia Institutional Animal Care and Use Committee. 12-16 week old female C57BL/6 mice were used for studies. A previously published non-splinted full thickness skin wound model ${ }^{326}$ was adapted and used for in vivo imaging trials. Briefly, mice were anesthetized with ketamine/xylazine/atropine $(60 / 4 / 0.2 \mathrm{mg} / \mathrm{kg})$ and the dorsum of the mice were depilated and sterilized. Mice were laid on their sides and dorsal skin was tented and pinned away from the body of the mouse to created a folded layer of skin. Three, three millimeter equidistant biopsy punches were created through the two layers of skin so as to create six, three millimeter full thickness skin wounds (Figure 7.2A). An analgesic (buprenorphine, $0.1 \mathrm{mg} / \mathrm{kg}$ ) was administered following surgery and the wounds were covered with a Tegaderm dressing.

\section{Imaging platform}

The CMOS camera (Point Grey Research, Grasshopper3 Camera) is a color CMOS digital camera capable of streaming 90 frames per second (fps) at 2048 x 2048 resolution. The camera was connected to a laptop (Lenovo ThinkPad W530) by a three meter USB 3.0 cable. For material characterization studies, the camera and lens (Spacecom one inch, f/0.95) were mounted $0.5 \mathrm{~m}$ directly above the sample and manually focused while the excitation source was placed next to the sample out of the camera's field of view. 
For in vivo studies, the camera was mounted to a Nikon Eclipse 80i equipped with an X-Cite 120 fluorescence light source filtered with a bandpass excitation filter $(360 / 20 \mathrm{~nm})$ and a longpass barrier filter (>425 nm). Analysis of RGB data was performed using a custom written MATLAB algorithms and scripts.

\section{In vivo imaging}

Mice were anesthetized with inhalable a $2 \%$ isoflurane/oxygen mixture and Tegaderm bandages were removed. Images of each wound under white light were taken using 20x magnification power to quantify wound area. Prior to imaging, wounds 1,3 , and 5 were superfused with $10 \mu \mathrm{L}$ of $5 \%$ dextrose solution, while wounds 2, 4, and 6 (right side) were superfused with ten microliters of $1 \mathrm{mg} / \mathrm{mL}$ BNPs (Figure 7.2A). Wounds 2, 4, and 6 were dosed before each imaging session. MJPEGs were acquired under UV illumination for each wound consisting of 1.) 5 frames (acquired at one frame per three seconds) of wound prior to application of $5 \%$ dextrose or BNPs, 2.) 60 frames (acquired at one frame per three seconds) of wounds after application of 5\% dextrose or BNPs, and 3.) 60 frames (acquired at one frame per three seconds) of wound after placing a sterile coverslip over wound to exclude ambient air.

\section{Wound area quantification}

Brightfield, 200x images of wounds were acquired at each time point and were imaged as described above. Image $J^{327}$ was used to quantify wound bed area at each 
day by manually tracing the wound bed and calculating the area. To keep measurements consistent, the periphery of the wound was traced at the outermost edge of the wound barrier in each image.

\section{Wound image processing}

The UV-illuminated wound images (acquired as described above) were analyzed using custom written MATLAB programs. Specific points within the wound bed were selected and the intensities of the red and blue color channels at those points were analyzed over the course of the image series. The background color intensities of the images at those points, at times prior to the addition of nanoparticles, were subtracted from the image series for all points within the image. As a result, any subsequent non-zero values for the red and blue channels were the result of nanoparticle fluorescence and phosphorescence only. The ratio of blue light intensity over red light intensity was computed for each pixel to represent the ratio of blue fluorescence (constant in the presence of BNPs) to red phosphorescence (quenched in the presence of oxygen). The upper and lower bounds for this ratio were set according the nanoparticles' sensitivity ranges. The ratiometric images were then displayed using a 256 -value color map scaled to the ratio bounds to spatiotemporally resolve fluorescence-to-phosphorescence ratios. 


\section{Results}

Iodide polymer exhibits distinct fluorescence and phosphorescence spectra

The dual-emissive iodide polymer used to make the BNPs has distinct fluorescence and phosphorescence emission spectra. The steady state spectra emission maximum under ambient air (21\% oxygen) is $459 \mathrm{~nm}$, while the delayed emission spectra maximum under nitrogen ( $0 \%$ oxygen) is $569 \mathrm{~nm}$ (Figure $7.1 \mathrm{~B}$ ). These spectrally separated fluorescence and phosphorescence maximum wavelengths make for simple detection of the emission peaks and lend themselves as a useful tool for oxygenation measurements by using ratiometry.

\section{Iodide polymer exhibits strong room temperature phosphorescence}

The iodide polymer used to make the BNPs exhibited strong room temperature phosphorescence, something that is rare for a biomaterial. Under nitrogen and UV excitation, the material showed a relative intensity $\mathrm{P} / \mathrm{F}$ of 9.1 and under ambient conditions (21\% oxygen) the $\mathrm{P} / \mathrm{F}$ was 1.2 (Figure 7.1B). This significant increase in the relative intensity under nitrogen caused a "light up" effect when the material was exposed to hypoxia. Under nitrogen (hypoxia) and UV excitation, the material emitted phosphorescence that was visible to the naked eye (Figure 7.1A). This strong room temperature phosphorescence in response to varying oxygen concentrations coupled with the distinct emission spectra of the fluorescence and phosphorescence make the iodide polymer an ideal oxygen-sensing material. 
BNPs exhibit linear fluorescence to phosphorescence ratios from $0-21 \%\left[\mathrm{O}_{2}\right]$ As described previously, the BNPs were exposed to various concentrations of oxygen to construct a ratiometric calibration curve. The nanoparticles exhibited a linear trend from $0-21 \%\left[\mathrm{O}_{2}\right]$ for both ratiometric intensity $\left(\mathrm{RI} ; \mathrm{R}^{2}=0.9956\right)$ and blue to red ratios $\left(\mathrm{RI} ; \mathrm{R}^{2}=0.9918\right)$ (Figure $7.1 \mathrm{C}$ ). The wide, linear range of ratiometric values $(\mathrm{F} / \mathrm{P})$ made the material ideal for physiological oxygen sensing applications.

\section{BNPs able to spatially resolve local oxygen levels}

Repeated application of BNPs to the wound bed allowed for effective differentiation of local oxygen levels and provided a spatial map of the oxygenation within the wound (Figure 7.2). After application of the BNPs to the wound bed (Figure 7.2A,B), UV excitation (Figure 7.2C) evoked a relatively stable blue channel signal within the wound bed, suggesting that the BNPs were evenly distributed throughout the wound bed (Figure 7.2D). A strong red channel signal (Figure 7.2E) was also emitted, which is akin to the phosphorescent emission. Application of $5 \%$ dextrose to the wounds served as the vehicle control and upon UV excitation emitted very little to no fluorescent or phosphorescent signal (data not shown). Following MATLAB processing as described previously, a spatial map within the wound bed was generated (Figure 7.2F). Figure 7.3 depicts a time course of the same wound and shows the changing oxygenation levels within the wound bed as the wound heals. 

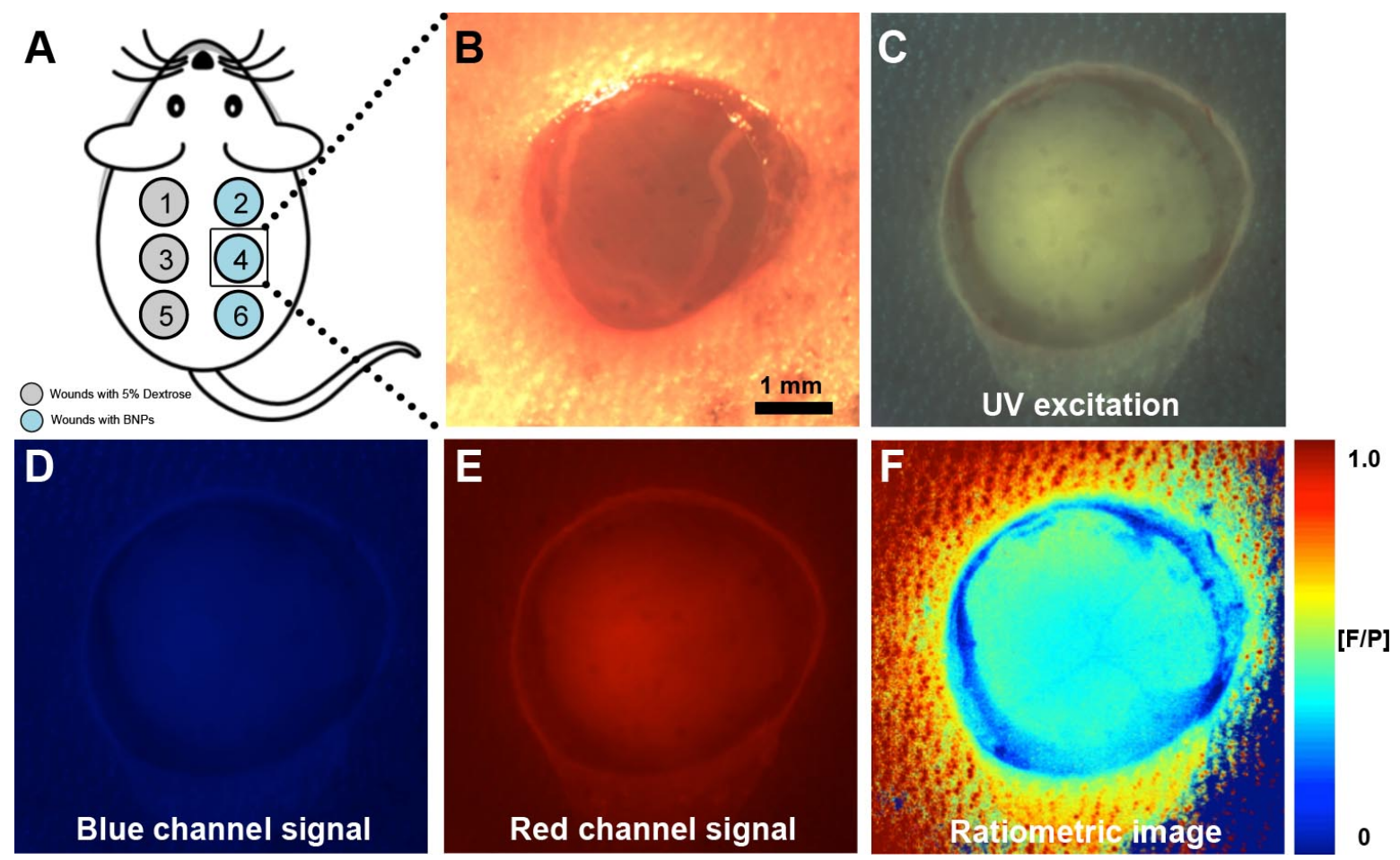

Figure 7.2: Application of BNPs to murine full thickness skin wound Ratiometric quantification of oxygenation levels within wound bed is possible by BNP application. A.) Wounds 2, 4, and 6 were superfused with BNPs while wounds 1, 3, and 5 were superfused with 5\% dextrose into $3 \mathrm{~mm}$ diameter wounds. B.) Brightfield image of full thickness skin wound. C.) Raw image of wound under UV excitation. D.) Blue channel luminescence (fluorescence). E.) Red channel luminescence (phosphorescence). F.) Processed RGB image with $F / P$ ratios performed by imaging platform.

\section{Wound bed oxygenation changes during healing}

The wound bed and wound boundaries appear to become less hypoxic as healing progresses, as evidenced by the red shift in the ratiometric images from Day 0 through Day 4 (Figure 7.3B,C). The red shift indicates a greater ratio of blue light to red, or increased fluorescence to phosphorescence, and is the result of the red phosphorescence being increasingly quenched in conditions of greater oxygen concentrations. This result can be visualized in both the uncovered and covered wound images (Figure 7.3B,C) and is consistent with expectations for a healthy 
wound, as processes necessary for wound healing, such as cell proliferation and collagen synthesis, require energy in the form of compounds whose syntheses are oxygen-dependent, such as ATP and $\mathrm{NADPH}^{296}$.

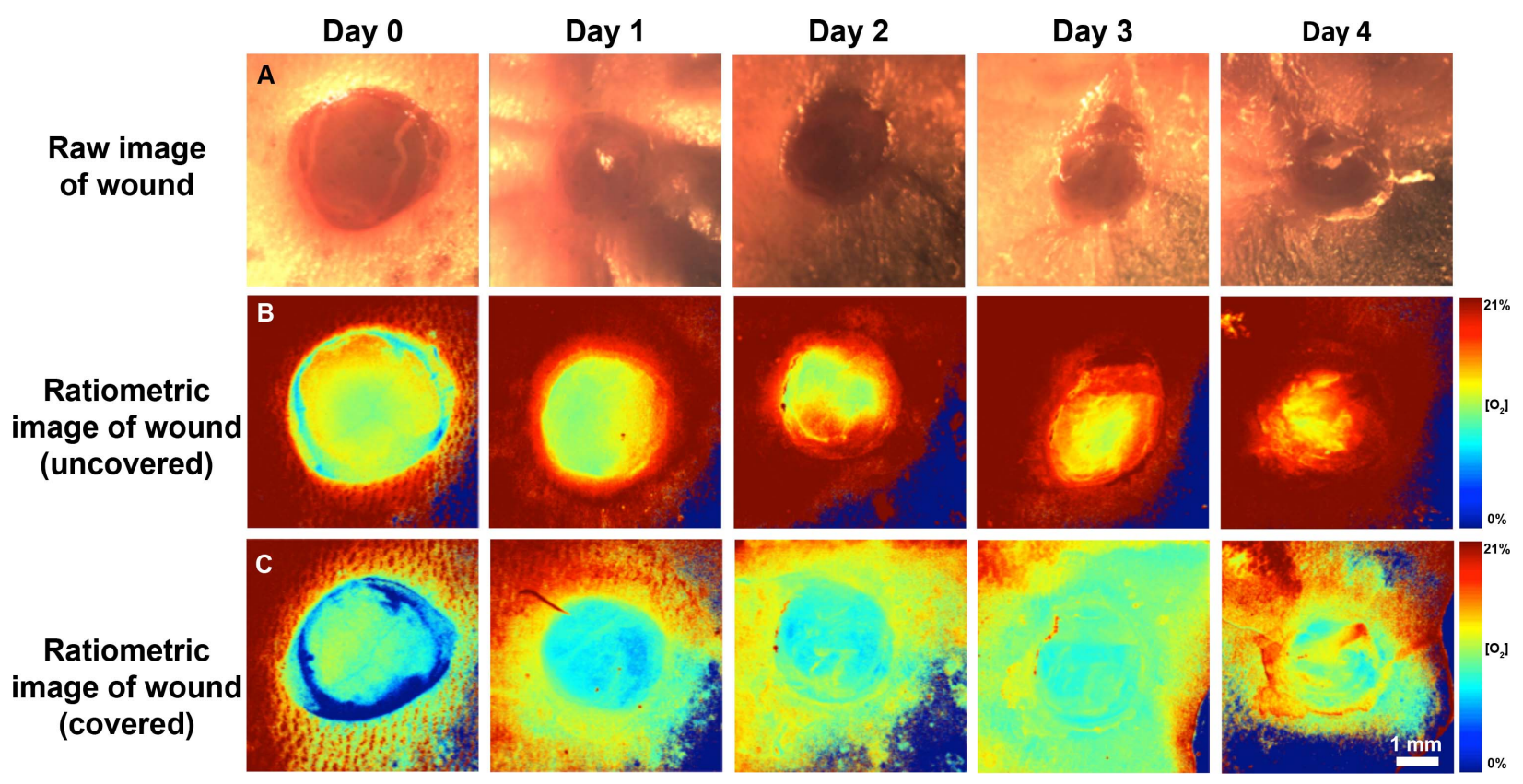

Figure 7.3: Healing time course and oxygenation levels within wound BNPs are able to spatially resolve oxygenation levels within wound bed. A.) Raw, brightfield image of wounds over the course of healing. B.) Uncovered ratiometric image acquired 5 minutes after BNP application. C.) Covered ratiometric image acquired 5 minutes after application of sterile glass coverslip.

Covering wounds reduces ambient oxygen levels near wounds and reduces resolution of oxygen distribution

Images of the BNP-treated wounds were captured both with and without a coverslip placed over the wound (Figure 7.3B,C) to determine the necessity of excluding ambient air prior to imaging. Other groups (as well as ours ${ }^{321}$ ) have used sterile coverslips or other barrier techniques to exclude ambient air in order to get an accurate oxygenation measurement within the wound bed. The application of the coverslips in these studies spread phosphorescence-quenched BNPs from the wound 
bed to the surrounding skin, resulting in a slight loss in resolution of both the wound boundaries and the oxygen distribution in the ratiometric images compared to the uncovered images (Figure 7.3B,C).

No difference in wound healing time for dextrose vs. NP application

Application of the BNPs did not delay wound healing as compared to application of the dextrose vehicle control (Figure 7.4). Healing curves were qualitatively similar between single applications of 5\% dextrose and single applications of BNPs as well as between repeated applications of 5\% dextrose and repeated applications of BNPs (Figure 7.4A). There was no statistically significant difference in the average area under the wound healing curves between single 5\% dextrose applications and single BNPs applications, or between repeated 5\% dextrose applications and repeated BNPs applications (Figure 7.4B).

Wound healing time of repeated versus single application of BNPs/5\% dextrose is not significantly different

Repeated application of BNPs or 5\% dextrose to the wound did not significantly slow wound healing when compared to single application of NPs or 5\% dextrose (one-way ANOVA, Tukey's post hoc test) (Figure 7.4A). The area under the curve for the repeated application of BNPs was slightly higher than single application of BNPs but was not significantly different (Figure 7.4B). Comparison of single 
application versus repeated application of $5 \%$ dextrose also did not reveal a significant difference in wound healing time.
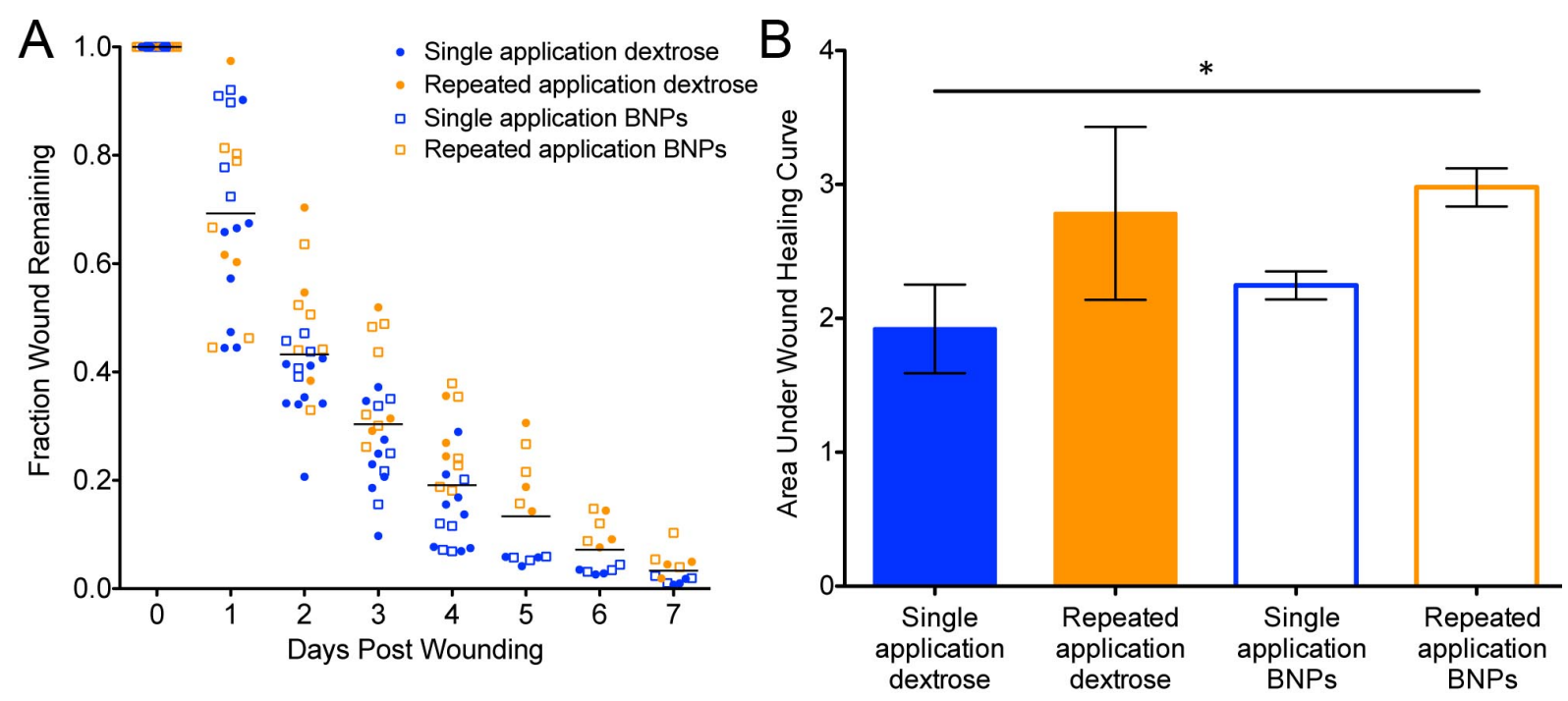

Figure 7.4: Quantification of wound healing time course

Application of BNPs and repeated dosing did not significantly slow healing time of wound. A) The fraction of the wound bed remaining after days of healing were quantified by normalizing wound area by the original wound area. B.) The area under the curve for each treatment was quantified to determine the effect of NPs on wound healing. One-way ANOVA and $P<0.05$ for single application dextrose and repeated application $B N P$ s. 


\section{Discussion}

Wound healing is directly related to oxygen levels within the wounds ${ }^{295}$. Tissue healing requires additional energy to facilitate increased extracellular matrix synthesis and cell proliferation ${ }^{296}$. The production of adenosine triphosphate (ATP) and other activated carrier molecules requires oxygen, so tissues are dependent on proper oxygenation to provide the energy to enable sufficient healing. Wounds that do not heal properly in an appropriate amount of time, due to an incomplete repair process, are known as chronic wounds. Therefore, monitoring wound oxygenation has garnered significant interest within the last 5 years $295,301,328-330$.

Much of the work focused on monitoring local wound oxygenation levels has made use of nano-probes as oxygen "sensors", with these nano-probes often being incorporated within a polymer matrix ${ }^{321,328,330}$. Li et al. showed the ability to resolve 2D oxygenation levels within a wound in a pre-clinical wound animal model by incorporating oxygen sensitive nano-probes in a paint-on bandage ${ }^{328}$. Schreml et al. developed oxygen-sensitive "sensor foils" to detect oxygenation levels in pre-clinical in vivo models that consisted of luminescent microparticles embedded within a polyurethane hydrogel that were spread thinly over poly(vinylidene-chloride) (PVdCl "Saran plastic wrap") ${ }^{330}$. Previous work by our group used oxygen-sensitive nanofibers embedded within a collagen gel to detect oxygenation levels of islet cells implanted into an in vivo mouse model ${ }^{321}$. All three of the aforementioned studies required the oxygen-sensing component (paint-on bandage, sensor foil, or nanofibers) to be isolated from ambient air $\left(\sim 21 \%\left[\mathrm{O}_{2}\right]\right)$ in order to quantify the oxygenation levels within the wound. To do so, these studies applied oxygen- 
limiting barriers (Saran plastic wrap) ${ }^{330}$. Tegaderm adhesive bandages ${ }^{328}$, or glass coverslips $^{321}$ over the wound for extended periods of time prior to imaging in order to mitigate oxygen exchange between the ambient air conditions and the oxygen sensors. Covering of the wounds in these studies may have artificially and transiently decreased the wound oxygenation level "sensed" and may not have been indicative of the actual state of the wound. To circumvent these limitations, our group made use of an aqueous NP solution that could be applied to topically to quantify oxygen levels.

To determine the ability of the aqueous BNP solution to resolve local wound oxygenation levels, we used an acute murine full thickness skin wound model ${ }^{326}$ in which we created six separate three millimeter wounds on the dorsum of the mouse and superfused the NPs solution or 5\% dextrose (vehicle control) into the individual wounds (Figures $7.2 \mathrm{~A}, \mathrm{~B}$ ). Using an image processing algorithm implemented in MATLAB, as described in the methods section, a spatial map of relative wound oxygenation levels was created (Figure 7.2F). Relative oxygen levels were visible, as the wound boundaries appeared more hypoxic than the center of the wound bed, in accordance with other materials used in the literature ${ }^{301,328,330}$. As illustrated by these studies, we believe that topical application of BNPs to a wound bed facilitates ratiometric quantification of local oxygenation levels, although we did not benchmark our measurements with the BNPs to any other "gold-standard" method, such as infrared spectroscopy (NIRS) or hyperspectral spectroscopy. 
As described above, many of the previously published oxygen nano-probes require the use of an oxygen impermeable barrier prior to imaging in order to limit oxygen diffusion from ambient air. As shown in Figure 7.3B, it appears that the BNPs are able to spatially resolve oxygenation levels within the wound bed without the need for an oxygen impermeable barrier. We wanted to compare uncovered ratiometric measurements to measurements taken in the presence of an oxygen impermeable barrier. Therefore, we amended our imaging protocol (above) to include an additional 60 frames (acquired at one frame per three seconds) after covering the wound margin with a sterile glass coverslip. As expected, the phosphorescent signal intensified over the course of image acquisition time period, as the wound area became more hypoxic due to the isolation of the wound from ambient air by the coverslip. In comparing the corresponding uncovered and covered images for each time point (Figure 7.3B,C), it was apparent that covering the wound area increased the phosphorescent signal (more hypoxic) and thus decreased the ratiometric values. Our results show an increase in phosphorescent signal following the application of the coverslip. We believe that the uncovered ratiometric measurements (Figure 7.3B) more accurately depict the oxygenation levels within the healing wound bed ${ }^{330}$, than do the covered ratiometric measurements (Figure 7.3C). Further, we believe that covering the wound with an oxygen impermeable coverslip falsely intensifies the phosphorescent signal, as evidenced by the several highly hypoxic areas observed in the spatial map (Figure 7.3C). Notably, what we believe to be artifact is also inconsistent with the wound 
healing observed three and four days post injury. To summarize, our images support that topically applied BNPs are able to resolve local oxygenation levels even in the absence of an air-impermeable coverslip.

Repeated application of the BNPs was necessary because the fluorescent and phosphorescent signals diminished to the level of being undetectable by our imaging technique upon the next imaging session. This attenuation in luminescence may have been due to boron de-coordination of the oxygen-sensitive material or to PLA degradation of the nanoparticles themselves ${ }^{322}$. Serial application of the BNPs and imaging of the wounds were performed each consecutive day for four days, and images were analyzed as described above. As expected, individual wound areas decreased as time progressed (Figure 7.3A) and oxygenation levels within the wound bed increased up to Day 4 (Figure 7.3B,C). Wound contracture and reepithelialization began to occur at Day 4, which is consistent with the woundhealing cascade. We observed a ring of low oxygenation at the wound periphery in both covered and uncovered images at Day 0 (Figure 7.3B,C). This observation is consistent with others in the field ${ }^{330}$ and we believe it is due to the clotting of blood around the excision site, as hemoglobin is excited at a similar wavelength.

Because PLA nanoparticles are known to have acidic byproducts (e.g. lactic acid) that may slow the wound healing process ${ }^{331}$, we assessed the potential detrimental effects of both single and repeated application of our BNPs. We calculated the size of the individual wound areas for wounds that were subjected to BNPs and for wounds that were subjected to $5 \%$ dextrose, which served as a vehicle 
control. Figure 7.4A plots the fraction of the wound remaining (i.e. not yet healed) when wounds were treated with BNPs (open, orange squares) vs. dextrose (closed, orange circles). When single and repeated doses were lumped together, there was no significant difference between the BNPs group and the control 5\% dextrose group with respect to wound healing over time. Figure $7.4 \mathrm{~B}$ graphs the average of the areas under the curve ${ }^{326}$ for each wound and reveals that there was no significant difference between repeated BNPs application and repeated 5\% dextrose application. Taken together, these data suggest that repeated application of the PLA nanoparticles does not deleteriously affect wound healing rates as compared to repeated application of $5 \%$ dextrose vehicle control. 


\section{Conclusions}

In these studies we presented a dual-emissive PLA based biomaterial that is fabricated into nanoparticles and calibrated for oxygen sensing applications. We coupled these nanoparticles with a cost-effective and portable imaging system to detect phosphorescence and fluorescence via RGB ratiometry techniques. This newly reported material, $\mathrm{BF}_{2} \mathrm{nbm}(\mathrm{I}) \mathrm{PLA}$, exhibited an unprecedented range of oxygen sensitivities from $0-21 \%\left[\mathrm{O}_{2}\right]$. The nanoparticles fabricated were able to spatially map oxygen levels within a murine full thickness skin wound and did not significantly impair wound healing time. The nanoparticles coupled with our custom-design imaging platform may be a powerful tool to monitor oxygenation levels in the clinical setting. Future work will be focused on these clinical applications, such as evaluation of oxygenation in keratinized healing skin, monitoring of a surgical flap after transfer, and documentation of injury/burn healing progression. 


\section{Chapter 8}

\section{Discussion AND FUTURE DIRECTIONS}




\section{Overview}

The unifying goal throughout this body of work was to enhance the volume retention of autologous fat for use in plastic and reconstructive surgery. To this end, we examined differing scales of autologous fat grafting ranging from large scale "flaps" to smaller scale "fat grafts". Proper vascularization of grafted autologous fat (both large and small scale) has long been considered to be the driving determinant of graft retention and thus we closely examined the microvasculature and its adaptations during autologous fat grafting.

The main work of this thesis focused on two current and emerging clinical realities of fat pre-treatment: flap delay prior to flap transfer (Chapters 2 and 3) and enzymatic digestion of fat grafts prior to implantation (Chapters 4 and 5). These pre-treatment strategies have been reported to increase graft survival and volume retention but several questions remained unanswered prior to our work. For example, during flap delay it was unknown what microvascular remodeling response was responsible for the observed increase in graft survival upon transfer. Additionally, the role of the immune system and its interactions with the remodeling vasculature was largely unexplored and unknown prior to our work. To the best of our knowledge we are the first to rigorously determine the interplay between microvascular remodeling and immune cell recruitment following flap delay. SVF supplementation of fat grafts at the small scale is a commonly practiced procedure, which has been reported to increase volume retention. However, it was largely unknown how the treatment of adipocytes/SVF with collagenase affects the viability of the cells themselves. We provide evidence that digestion of adipocytes 
and SVF cells is detrimental to their in vitro viability and confirmed this finding in vivo by using a xenograft model. We hope that our findings provide a better understanding of these pre-treatment techniques and will improve autologous fat grafting in the future.

This final chapter begins with a historical overview of tissue reconstruction and highlights the historical use of autologous fat in these reconstructive surgeries. I then summarize the contributions and extended applications of this work. I conclude this chapter with future directions that would be fruitful for further examination and propose several new exciting follow up experiments.

\section{History of reconstructive surgery}

Plastic and reconstructive surgery has an extensive history dating back to Ancient India (600 B.C.) with the oldest recorded instance being that of a nose reconstruction ${ }^{332}$. A Hindu surgeon, Sushruta, often reconstructed noses afflicted with battle wounds by using a flap transfer procedure of surrounding skin and fat ${ }^{332}$. The incidence of plastic surgery, specifically aesthetic surgeries, increased through Ancient Roman times ( 100 B.C.) likely due to the Roman's obsession with the beauty of the human body ${ }^{332}$. Through the middle ages, the occurrence of plastic surgery declined readily but was resurrected by the "father of modern plastic surgery", Gasparo Tagliacozzi, in the $16^{\text {th }}$ century ${ }^{30}$. Tagliacozzi, an Italian, experimented with skin flaps from the upper arms of patients and was the first to consider the vascular system and its implication in the survival of the flap upon 
transfer ${ }^{30}$. Tagliacozzi studied vascular pedicles within the upper arm skin flaps and recognized the importance of proper and sufficient blood flow to the flap ${ }^{30}$.

While the field of plastic surgery dates back many centuries, the use of autologous fat to reconstruct defects or improve aesthetic appeal is a rather new concept. The first recorded use of autologous fat was by Neuber in 1893 in which he filled facial scars with adipose tissue and reported that smaller pieces of adipose tissue led to better survival of the graft ${ }^{333}$. Autologous fat was reported to be used in rhinoplasty surgeries conducted by Brunning in 1911, breast reconstructive surgeries conducted by Lexer in 1931, and ophthalmological surgeries to reconstruct eyelids by Laubier in $1910^{333}$. Interestingly, Lyndon Peer reported a seminal finding in the 1950 s in which he stated that roughly $50 \%$ of adipose tissue volume that was grafted was resorbed by the end of the first year ${ }^{333,334}$. Surgical excision of adipose tissue was replaced with liposuction harvest of adipose tissue in the late $1970 \mathrm{~s}^{333}$ which further increased the use of autologous fat in reconstructive and aesthetic surgeries. While the use of autologous fat has increased over the past decade, the resorption rate reported by Peer et al. ${ }^{334}$ has not been mitigated and volume retention rates have not improved significantly with reports being as high as $80 \%{ }^{5}$.

Previous reports, including Tagliacozzi in the $16^{\text {th }}$ century, have recognized the importance of proper vascularization within the grafted tissue as a main determinant of survival of the graft ${ }^{14-17}$. Most previous studies have focused on the protein and cellular level to examine the vascularity of grafts and have not examined the microvascular network and network adaptations extensively. 
Further, the implications of the immune system during autologous fat grafting have not been studied significantly. In this thesis, the overarching goal was to examine the microvascular remodeling response and the contribution of the immune system on autologous fat survival.

\section{Contributions and extended applications}

Formulation of an in vivo murine model of flap delay

In this dissertation, I formulated a clinically relevant in vivo murine model of flap delay (Chapter 2) in which we rigorously characterized the microvascular remodeling responses within the flap. Previous pre-clinical studies examining flap delay have focused on changes in the cellular composition and protein levels within the flap ${ }^{33,34}$. Few studies, if any, have evaluated both angiogenesis and arteriogenesis within a flap at a vascular network level. In this dissertation, we evaluated angiogenesis and arteriogenesis within the delayed flap and determined that arteriogenesis predominates as the main vascular remodeling response. We extended our published model of flap delay and determined the mechanism underpinning vascular adaptations we observed in adipose tissue - the recruitment of anti-inflammatory macrophages. 

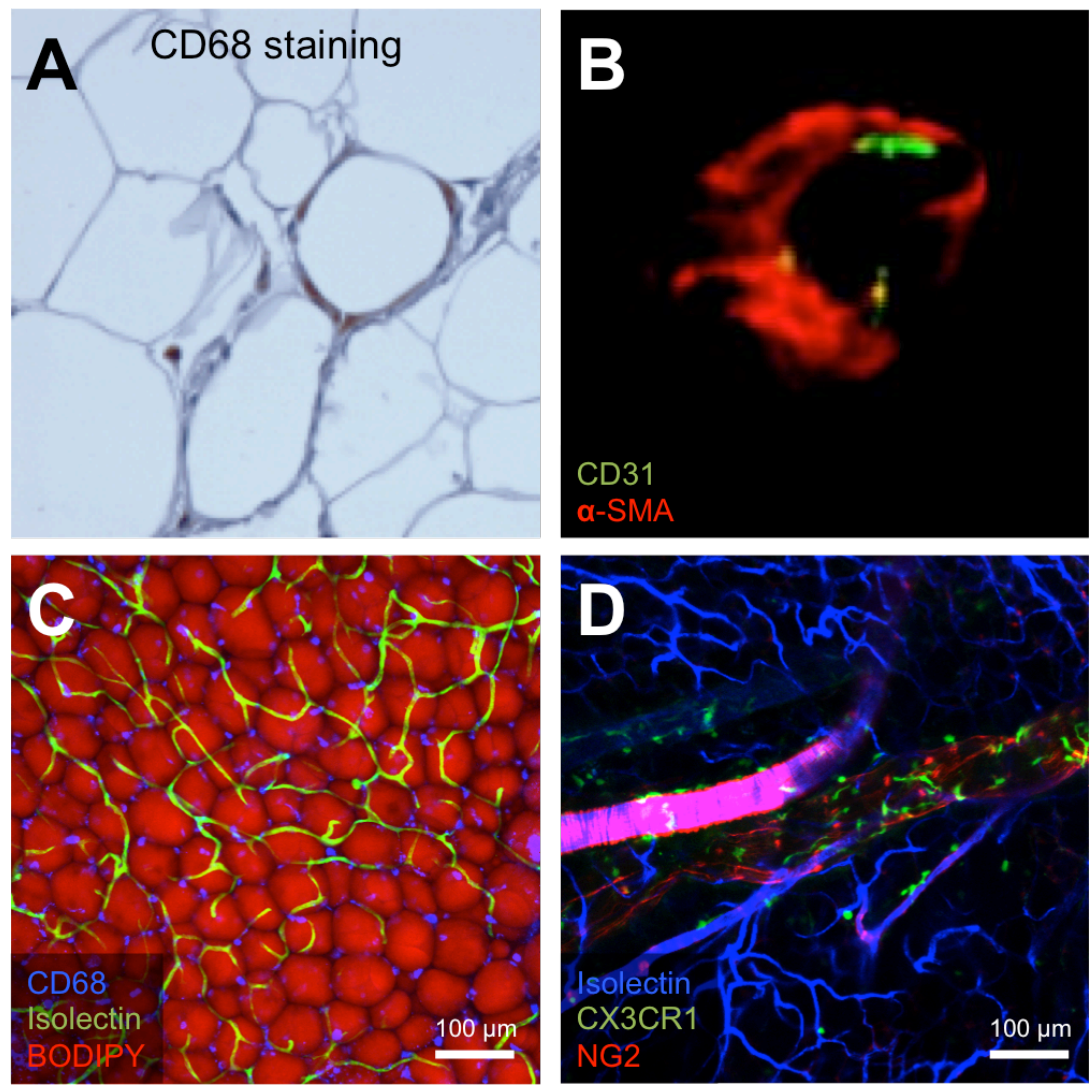

\section{Figure 8.1: Two-dimensional versus three-dimensional visualization of adipose tissue}

Three-dimensional visualization of adipose tissue allows for entire network visualization. A.) Sievers et al. two-dimensional image depicted $\mathrm{CD} 68^{+}$cells (macrophages in adipose tissue). ${ }^{335}$ B.) Lin et al. two-dimensional image of smooth muscle cells wrapping around capillary. ${ }^{336}$ C.) Our confocal micrograph z-stack (three-dimensional render) of vascular network within adipose tissue also depicting $C D 68^{+}$cells and adipocytes (BODIPY). D.) Our confocal micrograph z-stack (three-dimensional render) of vascular network and recruited monocytes $\left(\mathrm{CX} 3 \mathrm{CR} 1^{+}\right.$cells).

Three-dimensional network visualization of adipose tissue

Previous work examining the vasculature of adipose tissue has traditionally relied on cross section views to visualize the vascular network. While these techniques afford the ability to immunolabel cell types of interest, the spatial resolution is limited to two dimensions and a full image of the entire vascular network cannot be inferred (Figure 8.1A,B). Our work in this thesis thoroughly examined 
microvascular remodeling within adipose tissue, which is difficult to detect in two dimensions. Because of this specific need, I formulated a protocol to visualize vascular networks in adipose tissue by modifying our lab's whole mounting protocol of skeletal muscle. This technique afforded me a unique opportunity to visualize microvascular adaptations in three dimensions by constructing three-dimensional volume renders of confocal z-stacks, which allowed for characterization down to the capillary level (Figure 8.1C,D). Further, whole mounting of the adipose tissue allows one to capture the innate conformation of the vascular network, which was of extreme importance in Chapter 2 and 3 . We hope that this technique will be adopted and used by other labs that study vascular remodeling in adipose tissue.

\section{Implicate immune cells in vascular remodeling of adipose tissue}

The importance of recruited monocytes and anti-inflammatory macrophages during arteriogenesis has been well described in skeletal muscle ${ }^{86,138}$, but little to no work has been performed in adipose tissue in this regard. The use of our published model of flap delay provided us the ability to rigorously study the involvement of the immune system in the arteriogenic response observed. By using cell-specific reporter mice coupled with immunofluorescent staining, we were able to implicate the importance of recruited monocytes and anti-inflammatory macrophages during the vascular remodeling response. We are the first group to our knowledge that has studied the immune system and the effect on microvascular remodeling within adipose tissue. 
In Chapter 3 we extend our findings in Chapter 2 and confirm the role of anti-inflammatory macrophages in the remodeling response observed by performing a series of gain-of-function and loss-of-function studies. Clodronate liposome mediated depletion of macrophage populations within the inguinal fat pad abrogated the arteriogenic response observed in Chapter 2. We also demonstrate that direct addition of M2 macrophages and pharmacological recruitment of M2 macrophages increases the arteriogenic response observed in the inguinal fat pad. Taken together, these data confirm our findings in Chapter 2 - the importance of M2 macrophages in the remodeling response within a flap. Broadly speaking, these studies may suggest a mechanism for a translatable therapeutic to enhance the flap delay procedure.

Collagenase digestion has detrimental effect on adipocytes both in vitro and in vivo In Chapter 4, we reported that increasing durations of collagenase digestion in vitro were detrimental to adipocyte and interstitial cell viability of human and murine adipose tissue. Collagenase digestion is widely used to isolate the SVF and the adipocytes from human and murine adipose tissue ${ }^{8,24,144}$. However, the duration of enzymatic digestion in the literature is inconsistent and the effect of these varying durations was not previously explored. We are the first group, to our knowledge, who systematically correlated the effect of increased collagenase digestion with the viability of adipocytes and interstitial cells within murine and human adipose tissue. We found that increasing the duration of collagenase digestion decreased 
adipocyte viability, interstitial cell viability, and the total number of interstitial cells in both human and murine adipose tissue.

These findings have implications in both the plastic surgery and the laboratory setting. Collagenase digestion of adipose tissue is used extensively to harvest SVF and ASCs prior to "enhancing" fat grafts prior to implantation and a better understanding of the viability of these cells is of clinical importance ${ }^{8,259}$. Further, collagenase-digested adipocytes have been used as part of fat grafts so understanding the effect of collagenase digestion on adipocytes is of extreme importance in the clinic ${ }^{7,260}$. These findings are also of importance to laboratories that utilize adipocytes that have been enzymatically harvested to study the adipogenesis cascade 229,230 , adipokine secretion ${ }^{231-233}$, and adipocyte metabolism ${ }^{233}$.

In Chapter 5, we used a xenograft model of fat grafting ${ }^{141}$ to assess the effect of collagenase digestion on fat graft volume retention. Grafts that consisted of adipocytes that were collagenase digested prior to implantation had severely reduced graft volumes after twelve weeks post-implantation. Further, grafts consisting of collagenase-digested adipocytes had significant inflammation, fibrosis, and lower overall tissue health when compared to grafts that had undigested adipocytes. These studies indicate that collagenase digestion is detrimental to in vivo graft survival.

Taken together, these data provide caution to clinicians and scientists and suggest that collagenase digestion duration should be carefully controlled and the viability of cells harvested should be assessed prior to use. 


\section{Time ex vivo does not affect viability of adipose tissue}

In Chapter 4, we provide evidence that time ex vivo does not detrimentally affect adipocyte or interstitial cell viability in human or murine adipose tissue. In a clinical setting, there are often significant temporal delays between removal of the tissue from the donor site until implantation into the recipient site. This temporal delay also extends into the laboratory setting, where there can also be significant delays in removal of tissue from the organism until analysis of the tissue. In Chapter 4, we sequentially study the effect of time ex vivo on the viability of adipocytes by incubating human and murine adipose tissue up to two hours and assess viability of the adipose tissue. This finding will help inform clinicians and scientists of the effect of ex vivo incubation time of tissue samples prior to use or analysis.

\section{Novel assessment of paradoxical adipose hyperplasia tissue}

We were provided the unique opportunity to assess adipose tissue afflicted with paradoxical adipose hyperplasia (PAH) following CoolSculpting ${ }^{\circledR}$ (cryolipolysis) treatment. PAH is an extremely rare side effect of cryolipolysis treatment and the mechanism for the rapid expansion of adipose tissue is not well understood. Previous studies have mainly focused on the adipocytes and have not thoroughly examined the interstitial cellular component or microenvironment of $\mathrm{PAH}$ tissue $281,285,286$. We applied techniques optimized in Chapter 4 to evaluate the adipose tissue microenvironment (interstitial cells, adipocytes) of PAH tissue and 
control (untreated tissue). While further investigation into the mechanism of PAH is required, we provide evidence that the cellular microenvironment is disrupted and has fewer blood vessels and fewer interstitial cells. With the rising incidence of cryolipolysis, we have provided important evidence into this potential complication of cryolipolysis.

\section{Monitoring local wound oxygenation levels within tissue}

Monitoring oxygen levels within wounds, transferred tissue flaps, and burns is commonly practiced in the clinical, specifically in plastic surgery clinics. Plastic surgeons and other clinicians are often forced to determine if a wound or flap is healing. Traditional approaches to quantify oxygenation levels within a wound are expensive, rely on indirect quantification of oxygenation, and do not provide oxygenation levels within the entire wound bed ${ }^{296}$. In Chapter 7 , we report proof-ofconcept studies in which we demonstrated the ability of oxygen-sensitive nanoparticles ${ }^{317,320}$ to resolve local oxygenation levels within a murine wound bed. We presented a handheld custom-designed camera coupled with oxygen-sensitive nanoparticles as the first step toward their use as part of a clinical oxygen-sensing device.

\section{Future Directions}

Comprehensive examination of immune cell involvement in flap delay

Our results from Chapters 2 and 3 implicate macrophages, specifically antiinflammatory (M2) macrophages, as a major mediator of the observed arteriogenic 
response. These studies were founded on previous studies in our lab conducted in skeletal muscle in which we observed monocyte recruitment and anti-inflammatory macrophage accumulation around remodeling arterioles ${ }^{86,138}$. Arteriogenesis is a concerted bone marrow cell-directed inflammatory process that involved other types of immune cells such as T cells ${ }^{97,98}$, natural killer (NK) cells ${ }^{98}$, and neutrophils ${ }^{94,99}$. To our knowledge, no other studies have determined the interplay of these cell types and arteriogenesis in adipose tissue. While the work presented in this thesis focused on macrophages specifically, examination of these other immune cell types would be worthy of future investigation and may provide greater insight into the vascular remodeling process involved during flap delay.

\section{Optimization of collagenase digestion procedure prior to grafting}

In Chapter 4 we reported that the viability of adipocytes and interstitial cells decreases with increased duration of collagenase digestion. In Chapter 5 we tested the effect of collagenase digestion of the adipocytes on the long-term volume retention and described a significant decrease in the volume retention of grafts treated with collagenase prior to implantation. In Chapter 5 we tested one duration of collagenase digestion, 50 minutes. Accompanying our results in Chapter 4 that increasing collagenase digestion times decrease viability gradually over the course of 60 minutes, an important follow up study to consider shorter durations of collagenase digestion prior to implantation. It is possible that a 50 minute digestion is too harsh on the adipocytes and that a shorter digestion time is not detrimental 
to the viability of the adipocytes. Shorter durations of collagenase digestion may be beneficial to the survival of the graft by increasing the surface area to volume ratio and thus promoting more rapid revascularization of this tissue. This theory cannot be confirmed based on our current findings, but the effects of shorter durations of collagenase digestion on graft survival would certainly be of interest in future studies.

Another follow up study for the findings of Chapter 5 would be to titrate the concentration of SVF cells within the collagenase digested fat grafts. In Chapter 5 we used one concentration of SVF cells per fat graft based on previous findings by Paik et $\mathrm{al}^{254}$. As stated in the discussion of Chapter 5, it is possible that our SVF supplementation did not sufficiently "replenish" the interstitial cell compartment that was presumably removed during the digestion process. Increasing the number of SVF cells per fat graft may increase the volume retention of the graft and allow us to more accurately draw conclusions about the effect of collagenase digestion on in vivo graft survival. Other studies have examined the effect on volume retention by differing concentrations of SVF cells in the fat graft ${ }^{254}$, but to our knowledge no studies have not looked at this in conjunction with collagenase-digested fat grafts.

Mechanism responsible for collagenase-induced decreased viability and decreased volume retention

While we were able to show the detrimental effect of collagenase digestion both in vitro and in vivo, it is unclear what mechanism is responsible for the observed 
decrease in viability. As discussed in the discussion of Chapter 5 it is possible that collagenase digestion is inducing an inflammatory environment within the grafts mediated by TNF- $a^{267}$ and IL-6 ${ }^{268}$. It would be interesting to investigate the levels of these pro-inflammatory cytokines within the grafts, as we suspect that expression of these cytokines, among others, to be elevated in our grafts and may be the cause for decreased volume retention. Modulating these pro-inflammatory cytokines within the graft would be an interesting technique to improve graft retention. In that same vein, supplementing the grafts with anti-inflammatory cytokines to combat this inflammatory environment would be an interesting study to conduct in the future. Interestingly, Phipps et al. supplemented fat grafts with M2 macrophages and reported an increase in fat graft retention in a murine allograft model ${ }^{142}$. A better understanding of the effect of collagenase digestion on the proteins and cytokines within the adipose tissue may inform scientists and clinicians of ways to combat the decreased volume retention.

\section{Eliminate need for flap delay surgery}

Flap delay is an invasive, pre-treatment technique in which patients must visit the clinic one to two weeks prior to flap transfer in order to have the flap elevated/ligated. While this flap delay practice is indeed effective, the need for two separate surgical interventions is burdensome on the patient, clinician, and healthcare system. Our original goal when starting this work was to better understand the vascular changes within the flap following flap delay. With a sound 
understanding of this process, our ultimate goal was to determine if there was a way to recapitulate and induce these vascular changes without surgical intervention (i.e. the pre-treatment surgery).

This body of work did provide a better understanding of the changes induces by flap delay - an enlargement in collateral vessel diameters (arteriogenesis) accompanied by anti-inflammatory macrophages. FTY720, an FDA approved drug to treat multiple sclerosis, has been shown to induce arteriogenesis and antiinflammatory macrophage recruitment within skeletal muscle ${ }^{138,210}$. In Chapter 3 we report that delivery of FTY720 to sham inguinal fat pads increased the number of $\mathrm{CD} 206^{+}$cells and in ligated tissue FTY720 delivery increased the collateral vessel diameter and CD206 ${ }^{+}$cell number (Figure 3.4C,D). While delivery of FTY720 alone did not induce vascular changes within the inguinal fat pad, future optimization of FTY720 delivery may recapitulate the adaptation elicited by flap delay. Future studies could tailor the dosage/concentration of the drug as well as administration route (i.e. direct injection into fat pad) to determine if FTY720 alone could recapitulate the effects of flap delay. These results, coupled with previous findings ${ }^{138,210}$, provide exciting evidence that FTY720 may be used in a clinical setting. Eliminating the need for the pre-treatment surgery (flap delay) would be favorable for the health care system, specifically the patient and clinician. 
Noninvasive, serial tracking of microvascular remodeling

Photoacoustic microscopy (PAM) is an innovative tool that allows for label-free noninvasive high-resolution of structural vasculature architecture, relative oxygen saturation $\left(\mathrm{sO}_{2}\right)$, and blood flow velocity at the capillary level ${ }^{337,338}$. PAM has been used extensively to non-invasively observe vessel remodeling responses in the ear and brain ${ }^{337,339}$. This technique couples pulsed laser excitation of a tissue with ultrasound detection of the acoustic wave generated by the thermoelastic expansion to produce high-resolution images of the vasculature. The pulsed laser is often tuned to a wavelength that excites hemoglobin within the bloodstream, so as to provide contrast between the blood vessels and surrounding tissue.
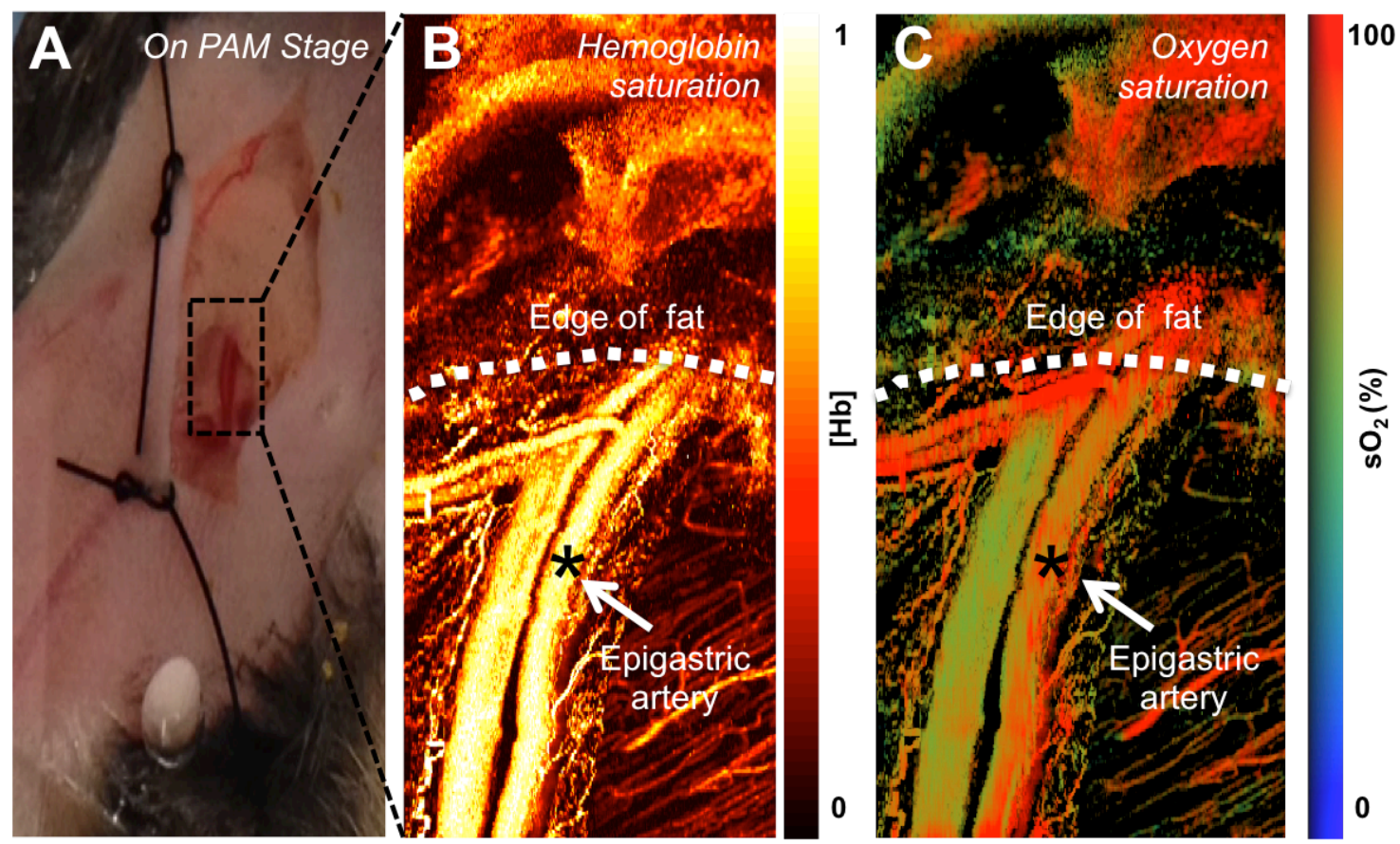

Figure 8.2: Photoacoustic microscopy of adipose tissue

Photoacoustic microscopy allows for visualization of epigastric artery entering the inguinal fat pad. A.) Macroscopic view of epigastric artery and vein pair entering inguinal fat pad. B.) Hemoglobin saturation visualization of epigastric artery and vein pair. Epigastric artery denoted by *. C.) Oxygen saturation visualization of epigastric artery and vein pair. Epigastric artery denoted by *. 
Using PAM within our flap delay model to serially and noninvasively track blood vessel architectural changes would be an interesting application and next step for this project. Previously, we performed proof-of-concept PAM studies within the adipose tissue in collaboration with Dr. Song Hu's laboratory and were able to visualize the epigastric artery (feeding vessel that is ligated in Chapters 2 and 3) entering the inguinal fat pad (Figure 8.2). We were able to quantify hemoglobin saturation (Figure 8.2B) and relative oxygen saturation (Figure 8.2C) However, visualization of the vessels within the fat pad itself proved difficult and requires further refinement in the imaging protocol. These proof-of-concept studies are exciting and provide evidence of the possibility of utilizing PAM in adipose tissue.

Our studies in Chapters 2 and 3 required the sacrifice of animals at specified time points and therefore we were not able to serially track the changes of specific blood vessels over time. A serial look at the adaptations within the vascular network may provide more insight into the remodeling process and would potentially allow us to draw conclusions about the time course of the remodeling process following flap delay.

\section{Advancement of oxygen-sensing device}

Admittedly, the BNP studies presented in this thesis are proof-of-concept studies, however; the results gathered are exciting and establish a basis for BNP use as part of an oxygen-sensing device. In Chapter 7, we applied BNPs to a full thickness skin wound of a mouse and were able to spatially resolve local oxygenation levels. The ultimate goal of this project is to formulate a clinically translatable oxygen-sensing 
device used by clinicians and nurses in a wound management or plastic surgery clinic. Oxygen levels within a wound or transferred flap are a powerful indicator of the ability of the wound or flap to heal properly 295,296 . Thus, we foresee this device being used to monitor proper oxygenation of evolving wounds/burns as well as used as a diagnostic tool to determine if a transferred flap is surviving.

The results following application of BNPs within the murine full thickness skin wound are indeed exciting and we believe that future work can be focused on several unknowns to strengthen the applicability of this technology in the clinic. For example, it is unknown if the BNPs are able to sense oxygenation levels below keratinized surfaces (intact skin) or whether the BNPs need to be directly applied to the wound or injury (as was the case in our studies of Chapter 7). We reliably and reproducibly showed the ability of BNPs to sense local oxygenation levels within a wound bed. Future studies should first be aimed at determining the feasibility of applying BNPs topically to keratinized surface. A simple feasibility test for question could be performed by topically applying BNPs to intact skin and subsequently restricting blood flow to this region via blood pressure cuff inflation. We could then measure the oxygen saturation with a pre-existing clinical device (oxygen saturation monitor) and compare these results to our spatial oxygen map garnered by the BNPs. The successful use of BNPs on keratinized surfaces would provide impetus for use of BNPs in many clinical presentations and applications.

Following flap transfer, hourly oxygenation monitoring of the newly transferred tissue is required to ensure that the flap is healing properly. This 
monitoring is typically performed by using oxygen saturation monitors, which provide only point measurements within the flap and do not provide a global map of the oxygenation within the tissue. Application of BNPs to transferred flaps may serve as a powerful tool to determine the health of the flap and may aid in the prediction of flap failure. Future studies could make use of the transverse rectus abdominis myocutaneous flap rat model in which the L-shaped flap is elevated and a subsequent, reproducible $\sim 40 \%$ loss of flap volume is observed ${ }^{340}$. Daily application of the BNPs to the elevated flap would provide spatial oxygenation maps of the flap and correlation of these maps with eventual necrosis and death of the flap would confirm ability of BNPs to predict flap failure. BNPs may also be of use for plastic surgeon or nurse when determining the depth of burn. It is often difficult for clinicians to predict the need for skin grafting following a burn injury because burns often afflict tissue much deeper than the surface of the skin. If BNPs can predict oxygenation levels below the skin surface they may serve as a powerful diagnostic and predictive tool for clinicians to determine the need for skin grafting. We could utilize a partial thickness scald burn murine model ${ }^{341}$ to determine the efficacy and feasibility of using BNPs to determine burn severity and depth.

We believe that further characterization and optimization of the oxygensensitive device will provide a powerful tool to clinicians and nurses. 


\section{Final remarks}

The goal of biomedical engineering is to design techniques and technologies to advance the treatment of disease and to better understand the human body. This thesis focused on the improvement of autologous fat grafting for use in reconstructive surgeries to repair soft tissue defects. A significant problem of using autologous fat to reconstruct these defects is the imminent and unpredictable resorption of the grafted fat. Clinicians and scientists have developed several pretreatment technique aimed to mitigate this resorption process, but significant advancements have not been achieved. In this thesis, I focused on understanding two pre-treatment techniques used in the clinic (flap delay and enzymatic digestion of grafts) and thoroughly examined the microvascular system in both cases. I first contributed a murine model of flap delay, which provided insight into the vascular remodeling processes as well as the involvement of the immune system. By using this model, I implicated anti-inflammatory macrophages as an integral part in these vascular adaptations and provide evidence for a translatable therapeutic mechanism to improve flap delay. I hope that this model will be useful in future studies aimed to improve the flap delay procedure. In a separate set of studies, I report the detrimental effect of collagenase digestion on fat grafts both in vitro and in vivo, something that was not explored previously before this dissertation but has large implications in a clinical setting. This work will hopefully provide cautionary evidence to clinicians and scientists who use collagenase-digested adipose tissue in the clinic or laboratory. In the latter part of this dissertation, I present the work in which I aided in the development of an oxygen-sensitive device that may ultimately 
be used in the clinic to diagnose and predict wound/burn healing progression and flap failure. In this thesis, I have presented many tools, techniques, and assays all aimed to improve autologous fat grafting in the clinical setting. I hope that my efforts contribute, at least in some part, to the ultimate goal of biomedical engineering: improvement of the treatment of disease and a better understanding of human health. 


\section{REFERENCES}

1. Bauer-Kreisel, P., Goepferich, A. \& Blunk, T. Cell-delivery therapeutics for adipose tissue regeneration. Adv. Drug Deliv. Rev. 62, 798-813 (2010).

2. The American Society of Plastic Surgeons. 2013 Reconstructive Demographics. (2013).

3. Trojahn Kølle, S.-F. et al. Importance of mesenchymal stem cells in autologous fat grafting: a systematic review of existing studies. J. Plast. Surg. Hand Surg. 46, 59-68 (2012).

4. Coleman, S. R. Structural fat grafting: more than a permanent filler. Plast. Reconstr. Surg. 118, 108S-120S (2006).

5. Kølle, S.-F. T. et al. Enrichment of autologous fat grafts with ex-vivo expanded adipose tissue-derived stem cells for graft survival: a randomised placebocontrolled trial. Lancet 382, 1113-20 (2013).

6. Fisher, C. et al. Comparison of harvest and processing techniques for fat grafting and adipose stem cell isolation. Plast. Reconstr. Surg. 132, 351-61 (2013).

7. Li, J. et al. Supplementing fat grafts with adipose stromal cells for cosmetic facial contouring. Dermatologic Surg. 39, 449-56 (2013).

8. Yoshimura, K. et al. Cell-assisted lipotransfer for cosmetic breast augmentation: supportive use of adipose-derived stem/stromal cells. Aesthetic Plast. Surg. 32, 48-55; discussion 56-7 (2008).

9. ISAPS. Cosmetic Procedures Performed in 2011. 1-11 (2011).

10. Peer, L. A. Loss of weight and volume in human fat grafts: with postulation of a 'cell survival theory'. Plast. Reconstr. Surg. 5, 217-230 (1950).

11. $\mathrm{Pu}, \mathrm{L}$. L. Q. Towards more rationalized approach to autologous fat grafting. $J$. Plast. Reconstr. Aesthet. Surg. 65, 413-9 (2012).

12. Kanchwala, S. K., Glatt, B. S., Conant, E. F. \& Bucky, L. P. Autologous fat grafting to the reconstructed breast: the management of acquired contour deformities. Plast. Reconstr. Surg. 124, 409-18 (2009).

13. Sherman, J. E., Fanzio, P. M., White, H. \& Leifer, D. Blindness and necrotizing fasciitis after liposuction and fat transfer. Plast. Reconstr. Surg. 126, 1358-63 (2010).

14. Carpaneda, C. A. \& Ribeiro, M. T. Study of the histologic alterations and viability of the adipose graft in humans. Aesthetic Plast. Surg. 17, 43-7 (1993).

15. Carpaneda, C. A. \& Ribeiro, M. T. Percentage of graft viability versus injected volume in adipose autotransplants. Aesthetic Plast. Surg. 18, 17-19 (1994). 
16. Nishimura, T., Hashimoto, H., Nakanishi, I. \& Furukawa, M. Microvascular angiogenesis and apoptosis in the survival of free fat grafts. Laryngoscope 110, 1333-8 (2000).

17. Hamed, S. et al. Treating fat grafts with human endothelial progenitor cells promotes their vascularization and improves their survival in diabetes mellitus. Plast. Reconstr. Surg. 130, 801-11 (2012).

18. Vecchio, D. Del \& Fichadia, H. in Breast Reconstruction: Current Techiques (2012). at <http://cdn.intechopen.com/pdfs/27951/InTechAutologous_fat_transplantation_a_paradigm_shift_in_breast_reconstruction.p df $>$

19. Mathes, S. J. \& Nahai, F. Classification of the vascular anatomy of muscles: experimental and clinical correlation. Plastic and reconstructive surgery 67, 177-187 (1981).

20. Cui, X. \& Pu, L. L. Q. The Search for a Useful Method for the Optimal Cryopreservation of Adipose Aspirates: Part II. In Vivo Study. Aesthetic Surg. J. 30, 451-456 (2010).

21. Gimble, J. M., Bunnell, B. a., Chiu, E. S. \& Guilak, F. Concise review: Adipose-derived stromal vascular fraction cells and stem cells: Let's not get lost in translation. Stem Cells 29, 749-754 (2011).

22. Bourin, P. et al. Stromal cells from the adipose tissue-derived stromal vascular fraction and culture expanded adipose tissue-derived stromal/stem cells: a joint statement of the International Federation for Adipose Therapeutics and Science (IFATS) and the International So. Cytotherapy 15, 641-648 (2013).

23. Gimble, J. M., Katz, A. J. \& Bunnell, B. a. Adipose-derived stem cells for regenerative medicine. Circ. Res. 100, 1249-60 (2007).

24. Gimble, J. M. \& Guilak, F. Adipose-derived adult stem cells: isolation, characterization, and differentiation potential. Cytotherapy 5, 362-369 (2003).

25. Shah, F. S., Wu, X., Dietrich, M., Rood, J. \& Gimble, J. M. A non-enzymatic method for isolating human adipose tissue-derived stromal stem cells. Cytotherapy 15, 979-985 (2013).

26. Raposio, E., Caruana, G., Bonomini, S. \& Libondi, G. A novel and effective strategy for the isolation of adipose-derived stem cells: minimally manipulated adipose-derived stem cells for more rapid and safe stem cell therapy. Plast. Reconstr. Surg. 133, 1406-9 (2014).

27. Dong, Z. et al. In vivo injectable human adipose tissue regeneration by adipose-derived stem cells isolated from the fluid portion of liposuction aspirates. Tissue Cell 46, 178-184 (2014). 
28. Bunnell, B. A., Estes, B. T., Guilak, F. \& Gimble, J. M. Differentiation of adipose stem cells. Methods Mol. Biol. 456, 155-71 (2008).

29. Eisenhardt, S. U. et al. Monitoring molecular changes induced by ischemia/reperfusion in human free muscle flap tissue samples. Ann. Plast. Surg. 68, 202-8 (2012).

30. Santoni-Rugiu, P. \& Sykes, P. A History of Plastic Sugery. (2007).

31. Ghali, S., Butler, P. E. M., Tepper, O. M. \& Gurtner, G. C. Vascular delay revisited. Plast. Reconstr. Surg. 119, 1735-44 (2007).

32. Wong, M. S. et al. Basic Fibroblast Growth Factor Expression following Surgical Delay of Rat Transverse Rectus Abdominis Myocutaneous Flaps. Plast. Reconstr. Surg. 113, 2030-2036 (2004).

33. Lineaweaver, W. C. et al. Vascular Endothelium Growth Factor, Surgical Delay, and Skin Flap Survival. Ann. Surg. 239, 866-875 (2004).

34. Ceradini, D. J. et al. Progenitor cell trafficking is regulated by hypoxic gradients through HIF-1 induction of SDF-1. Nat. Med. 10, 858-64 (2004).

35. Park, S. et al. Selective recruitment of endothelial progenitor cells to ischemic tissues with increased neovascularization. Plast. Reconstr. Surg. 113, 284-93 (2004).

36. Butala, P. et al. Endogenous stem cell therapy enhances fat graft survival. Plast. Reconstr. Surg. 130, 293-306 (2012).

37. Condé-Green, A. et al. Comparison of 3 techniques of fat grafting and cellsupplemented lipotransfer in athymic rats: a pilot study. Aesthet. Surg. J. 33, 713-21 (2013).

38. Bateman, R. M., Sharpe, M. D. \& Ellis, C. G. Bench-to-bedside review: Microvascular dysfunction in sepsis - hemodynamics, oxygen transport, and nitric oxide. Crit. Care 359-373 (2003). doi:10.1186/cc2353

39. Granger, D. N. \& Senchenkova, E. Inflammation and the Microcirculation. Colloquium Series on Integrated Systems Physiology: From Molecule to Function 2, (2010).

40. Yuan, S. Y. \& Rigor, R. R. Regulation of Endothelial Barrier Function. Colloq. Ser. Integr. Syst. Physiol. From Mol. to Funct. 3, 1-146 (2011).

41. Wiedeman, M. P. Dimensions of Blood Vessels from Distributing Artery to Collecting Vein. Circ. Res. 12, 375-378 (1963).

42. Sandoo, A., van Zanten, J. J. C. S. V., Metsios, G. S., Carroll, D. \& Kitas, G. D. The endothelium and its role in regulating vascular tone. Open Cardiovasc. Med. J. 4, 302-312 (2010).

43. Borisova, L., Wray, S., Eisner, D. A. \& Burdyga, T. How Structure, Ca Signals, and Cellular Communications Underlie Function in Precapillary Arterioles. Circ. Res. 105, 803-810 (2009). 
44. Cines, B. D. B. et al. The Journal of The American Society of Hematology. J. Am. Soc. Hematol. 91, 3527-3561 (1998).

45. Armulik, A., Genové, G. \& Betsholtz, C. Pericytes: Developmental, Physiological, and Pathological Perspectives, Problems, and Promises. Dev. Cell 21, 193-215 (2011).

46. Shepro, D. \& Morel, N. Pericyte Physiology. FASEB J. 1031-1038 (1993).

47. Bergers, G. \& Song, S. The role of pericytes in blood-vessel formation and maintenance. Neuro. Oncol. 7, 452-464 (2005).

48. Kelly-Goss, M. R., Sweat, R. S., Stapor, P. C., Peirce, S. M. \& Murfee, W. L. Targeting Pericytes for Angiogenic Therapies. Microcirculation 21, 345-357 (2014).

49. Stark, K. et al. Capillary and arteriolar pericytes attract innate leukocytes exiting through venules and 'instruct' them with pattern-recognition and motility programs. Nat. Immunol. 14, 41-51 (2013).

50. Peppiatt, C. M., Howarth, C., Mobbs, P. \& Attwell, D. Bidirectional control of CNS capillary diameter by pericytes. Nature 443, 700-704 (2006).

51. Hirschi, K. K., Rohovsky, S. A., Beck, L. H., Smith, S. R. \& D’Amore, P. A. Endothelial Cells Modulate the Proliferation of Mural Cell Precursors via Platelet-Derived Growth Factor-BB and Heterotypic Cell Contact. Circ. Res. 84, 298-305 (1999).

52. Hellstrom, M. et al. Lack of Pericytes Leads to Endothelial Hyperplasia and Abnormal Vascular Morphogenesis. J. Cell Biol. 153, 543-554 (2001).

53. Lauridsen, H. M., Pober, J. S. \& Gonzalez, A. L. A composite model of the human postcapillary venule for investigation of microvascular leukocyte recruitment. FASEB J. 28, 1166-1180 (2014).

54. Dvorak, H. F. Vascular permeability to plasma, plasma proteins, and cells: an update. Curr. Opin. Hematol. 1 (2010). doi:10.1097/MOH.0b013e3283386638

55. Murfee, W. L., Skalak, T. C. \& Peirce, S. M. Differential Arterial/Venous Expression of NG2 Proteoglycan in Perivascular Cells Along Microvessels: Identifying a Venule-Specific Phenotype. Microcirculation 12, 151-160 (2005).

56. Ellis, C. G., Jagger, J. \& Sharpe, M. The microcirculation as a functional system. Crit. Care 9, S3 (2005).

57. Peirce, S. M. \& Skalak, T. C. Microvascular remodeling: a complex continuum spanning angiogenesis to arteriogenesis. Microcirculation 10, 99-111 (2003).

58. Waters, R. E., Rotevatn, S., Li, P., Annex, B. \& Yan, Z. Voluntary running induces fiber type-specific angiogenesis in mouse skeletal muscle. AJP Cell Physiol. 287, C1342-C1348 (2004).

59. Kota, S. et al. Aberrant angiogenesis: The gateway to diabetic complications. Indian J. Endocrinol. Metab. 16, 918 (2012). 
60. Wilkinson-Berka, J. Vasoactive Factors and Diabetic Retinopathy: Vascular Endothelial Growth Factor, Cycoloxygenase-2 and Nitric Oxide. Curr. Pharm. Des. 10, 3331-3348 (2004).

61. Hanahan, D. \& Weinberg, R. A. Hallmarks of Cancer: The Next Generation. Cell 144, 646-674 (2011).

62. Carmeliet, P. Angiogenesis in health and disease. Nat. Med. 9, 653-660 (2003).

63. Deveci, D., Marshall, J. M. \& Egginton, S. Chronic hypoxia induces prolonged angiogenesis in skeletal muscles of rat. Exp. Physiol. 87, 287-91 (2002).

64. Hadjipanayi, E. \& Schilling, A. F. Hypoxia-based strategies for angiogenic induction. Organogenesis 9, 261-272 (2013).

65. Krock, B. L., Skuli, N. \& Simon, M. C. Hypoxia-Induced Angiogenesis: Good and Evil. Genes Cancer 2, 1117-1133 (2011).

66. Wahlberg, E. Angiogenesis and arteriogenesis in limb ischemia. J. Vasc. Surg. 38, 198-203 (2003).

67. Tang, G. L., Chang, D. S., Sarkar, R., Wang, R. \& Messina, L. M. The effect of gradual or acute arterial occlusion on skeletal muscle blood flow, arteriogenesis, and inflammation in rat hindlimb ischemia. J. Vasc. Surg. 41, $312-320$ (2005).

68. Heil, M. \& Schaper, W. Influence of Mechanical, Cellular, and Molecular Factors on Collateral Artery Growth (Arteriogenesis). Circ. Res. 95, 449-458 (2004).

69. Heil, M., Eitenmüller, I., Schmitz-Rixen, T. \& Schaper, W. Arteriogenesis versus angiogenesis: similarities and differences. J. Cell. Mol. Med. 10, 45-55 (2006).

70. Vailhé, B., Vittet, D. \& Feige, J. J. In vitro models of vasculogenesis and angiogenesis. Lab. Invest. 81, 439-452 (2001).

71. Djonov, V., Schmid, M., Tschanz, S. A. \& Burri, P. H. Intussusceptive Angiogenesis : Its Role in Embryonic Vascular Network Formation. Circ. Res. 86, 286-292 (2000).

72. Patan, S., Munn, L. L. \& Jain, R. K. Intussusceptive Microvascular Growth in a Human Colon Adenocarcinoma Xenograft: A Novel Mechanism of Tumor Angiogenesis. Microvasc. Res. 51, 260-272 (1996).

73. Patan, S., Alvarez, M. J., Schittny, J. C. \& Burri, P. H. Intussusceptive Microvascular Growth: A Common Alternative to Capillary Sprouting. Arch. Histol. Cytol. 55, 65-75 (1992).

74. Wietecha, M. S., Cerny, W. L. \& DiPietro, L. A. in 3-32 (2012). doi:10.1007/82_2012_287 
75. Eilken, H. M. \& Adams, R. H. Dynamics of endothelial cell behavior in sprouting angiogenesis. Curr. Opin. Cell Biol. 22, 617-625 (2010).

76. Potente, M., Gerhardt, H. \& Carmeliet, P. Basic and Therapeutic Aspects of Angiogenesis. Cell 146, 873-887 (2011).

77. Breier, G. Angiogenesis in Embryonic Development-A Review. Placenta 21, S11-S15 (2000).

78. Harada, H. et al. Significance of HIF-1-active cells in angiogenesis and radioresistance. Oncogene 26, 7508-7516 (2007).

79. Forsythe, J. A. et al. Activation of vascular endothelial growth factor gene transcription by hypoxia-inducible factor 1. Mol. Cell. Biol. 16, 4604-4613 (1996).

80. Hellström, M. et al. Dll4 signalling through Notch1 regulates formation of tip cells during angiogenesis. Nature 445, 776-780 (2007).

81. Walpole, J. et al. Agent-based model of angiogenesis simulates capillary sprout initiation in multicellular networks. Integr. Biol. 7, 987-997 (2015).

82. Liu, Z.-J. et al. Regulation of Notch1 and Dll4 by Vascular Endothelial Growth Factor in Arterial Endothelial Cells: Implications for Modulating Arteriogenesis and Angiogenesis. Mol. Cell. Biol. 23, 14-25 (2003).

83. Taylor, A. C., Seltz, L. M., Yates, P. a \& Peirce, S. M. Chronic whole-body hypoxia induces intussusceptive angiogenesis and microvascular remodeling in the mouse retina. Microvasc. Res. 79, 93-101 (2010).

84. Guendel, A. M. et al. Murine spinotrapezius model to assess the impact of arteriolar ligation on microvascular function and remodeling. J. Vis. Exp. e50218 (2013). doi:10.3791/50218

85. Mac Gabhann, F., Peirce, S. M. S. M., Gabhann, F. Mac \& Peirce, S. M. S. M. Collateral Capillary Arterialization following arteriolar ligation in murine skeletal muscle. Microcirculation 17, 333-347 (2010).

86. Bruce, A. C. et al. Monocytes are recruited from venules during arteriogenesis in the murine spinotrapezius ligation model. Arterioscler. Thromb. Vasc. Biol. 1-11 (2014). doi:10.1161/ATVBAHA.114.303399

87. Heil, M. et al. Collaretal Artery Growth (Arteriogenesis) after Experimental Arterial Occlusion Is Impaired in Mice Lacking CC-Chemokine Receptor-2. Circ. Res. 94, 671-677 (2004).

88. Schaper, W. \& Scholz, D. Factors Regulating Arteriogenesis. Arterioscler. Thromb. Vasc. Biol. 23, 1143-1151 (2003).

89. Lee, C. W. et al. Temporal Patterns of Gene Expression after Acute Hindlimb Ischemia in Mice: Insights into the Genomic Program for Collateral Vessel Development. J. Am. Coll. Cardiol. 43, 474-482 (2004). 
90. Hoefer, I. E. et al. Arteriogenesis Proceeds via ICAM-1/Mac-1- Mediated Mechanisms. Circ. Res. 94, 1179-1185 (2004).

91. van Royen, N. et al. Stimulation of arteriogenesis; a new concept for the treatment of arterial occlusive disease. Cardiovasc. Res. 49, 543-553 (2001).

92. Scholz, D. et al. Ultrastructure and molecular histology of rabbit hind-limb collateral artery growth (arteriogenesis). Virchows Arch. 436, 257-70 (2000).

93. Arras, M. et al. Monocyte activation in angiogenesis and collateral growth in the rabbit hindlimb. J. Clin. Invest. 101, 40-50 (1998).

94. Meisner, J. K. \& Price, R. J. Spatial and Temporal Coordination of Bone Marrow-Derived Cell Activity during Arteriogenesis: Regulation of the Endogenous Response and Therapeutic Implications. Microcirculation 17, 583-599 (2010).

95. Schaper, W. \& Scholz, D. Factors Regulating Arteriogenesis. Arterioscler. Thromb. Vasc. Biol. 23, 1143-1151 (2003).

96. Ziegelhoeffer, T. et al. Bone Marrow-Derived Cells Do Not Incorporate into the Adult Growing Vasculature. Circ. Res. 94, 230-238 (2004).

97. Stabile, E. et al. Impaired Arteriogenic Response to Acute Hindlimb Ischemia in CD4-Knockout Mice. Circulation 108, 205-211 (2003).

98. van Weel, V. et al. Natural killer cells and CD4+ T-cells modulate collateral artery development. Arterioscler. Thromb. Vasc. Biol. 27, 2310-8 (2007).

99. Soehnlein, O. et al. Neutrophil secretion products pave the way for inflammatory monocytes. Blood 112, 1461-1471 (2008).

100. Heissig, B. et al. Low-dose irradiation promotes tissue revascularization through VEGF release from mast cells and MMP-9-mediated progenitor cell mobilization. J. Exp. Med. 202, 739-750 (2005).

101. Carmeliet, P. Mechanisms of angiogenesis and arteriogenesis. Nature 6, 389395 (2000).

102. Bailey, A. M., O’Neill, T. J., Morris, C. E. \& Peirce, S. M. Arteriolar remodeling following ischemic injury extends from capillary to large arteriole in the microcirculation. Microcirculation 15, 389-404 (2008).

103. Sethi, J. K. \& Vidal-Puig, A. J. Thematic review series: adipocyte biology. Adipose tissue function and plasticity orchestrate nutritional adaptation. $J$. Lipid Res. 48, 1253-62 (2007).

104. Trayhurn, P. \& Beattie, J. H. Physiological role of adipose tissue: white adipose tissue as an endocrine and secretory organ. Proc. Nutr. Soc. 60, 329339 (2001).

105. Trayhurn, P., Bing, C. \& Wood, I. S. Adipose tissue and adipokines - energy regulation from the human perspective. J. Nutr. 136, 1935S-1939S (2006). 
106. Ouchi, N., Parker, J. L., Lugus, J. J. \& Walsh, K. Adipokines in inflammation and metabolic disease. Nat. Rev. Immunol. 11, 85-97 (2011).

107. Caruso, C., Balistreri, C. R. \& Candore, G. The role of adipose tissue and adipokines in obesity-related inflammatory diseases. Mediators Inflamm. (2010). doi:10.1155/2010/802078

108. Harms, M. \& Seale, P. Brown and beige fat: development, function and therapeutic potential. Nat. Med. 19, 1252-1263 (2013).

109. Rosell, M. et al. Brown and white adipose tissues: intrinsic differences in gene expression and response to cold exposure in mice. Am. J. Physiol. Endocrinol. Metab. 306, E945-64 (2014).

110. van der Lans, A. a J. J. et al. Cold acclimation recruits human brown fat and increases nonshivering thermogenesis. J. Clin. Invest. 123, 3395-3403 (2013).

111. Gilsanz, V., Hu, H. H. \& Kajimura, S. Relevance of brown adipose tissue in infancy and adolescence. Pediatr. Res. 73, 3-9 (2012).

112. Atzmon, G. et al. Differential Gene Expression Between Visceral and Subcutaneous Fat Depots. Horm. Metab. Res. 34, 622-628 (2002).

113. Lee, M.-J., Wu, Y. \& Fried, S. K. Adipose tissue heterogeneity: Implication of depot differences in adipose tissue for obesity complications. Mol. Aspects Med. 34, 1-11 (2013).

114. Gu, P. \& Xu, A. Interplay between adipose tissue and blood vessels in obesity and vascular dysfunction. Rev. Endocr. Metab. Disord. 14, 49-58 (2013).

115. Gregoire, F. M., Smas, C. M. \& Sul, H. S. Understanding adipocyte differentiation. Physiol. Rev. 78, 783-809 (1998).

116. Ferrante, a W. The immune cells in adipose tissue Innate Immune Cell Populations in Adipose Tissue. Diabetes, Obes. Metab. 15, 34-38 (2013).

117. Mraz, M. \& Haluzik, M. The role of adipose tissue immune cells in obesity and low-grade inflammation. J. Endocrinol. 1-35 (2014). doi:10.1530/JOE-14-0283

118. Gordon, S. \& Taylor, P. R. Monocyte and macrophage heterogeneity. Nat. Rev. Immunol. 5, 953-964 (2005).

119. van Furth, R. et al. The mononuclear phagocyte system: a new classification of macrophages, monocytes, and their precursor cells. Bull. World Health Organ. 46, 845-852 (1972).

120. Hume, D. A. The mononuclear phagocyte system. Curr. Opin. Immunol. 18, 49-53 (2006).

121. Corliss, B. A., Azimi, M. S., Munson, J., Peirce, S. M. \& Murfee, W. L. Macrophages: An Inflammatory Link between Angiogenesis and Lymphangiogenesis. Microcirculation (2015).

122. Randolph, G. J., Jakubzick, C. \& Qu, C. Antigen presentation by monocytes and monocyte-derived cells. Curr. Opin. Immunol. 20, 52-60 (2008). 
123. Soehnlein, O., Lindbom, L. \& Weber, C. Mechanisms underlying neutrophilmediated monocyte recruitment. Blood 114, 4613-4623 (2009).

124. Houghton, A. M., Hartzell, W. O., Robbins, C. S., Gomis-Rüth, F. X. \& Shapiro, S. D. Macrophage elastase kills bacteria within murine macrophages. Nature (2009). doi:10.1038/nature08181

125. Henke, C. et al. Macrophage production of basic fibroblast growth factor in the fibroproliferative disorder of alveolar fibrosis after lung injury. Am. J. Pathol. 143, 1189-1199 (1993).

126. Sunderkötter, C., Goebeler, M., Schulze-Osthoff, K., Bhardwaj, R. \& Sorg, C. Macrophage-derived angiogenesis factors. Pharmacol. Ther. 51, 195-216 (1991).

127. Arango Duque, G. \& Descoteaux, A. Macrophage Cytokines: Involvement in Immunity and Infectious Diseases. Front. Immunol. 5, (2014).

128. Pozzi, L.-A. M., Maciaszek, J. W. \& Rock, K. L. Both dendritic cells and macrophages can stimulate naive CD8 T cells in vivo to proliferate, develop effector function, and differentiate into memory cells. J. Immunol. 175, 20712081 (2005).

129. Hashimoto, D. et al. Tissue-Resident Macrophages Self-Maintain Locally throughout Adult Life with Minimal Contribution from Circulating Monocytes. Immunity 38, 792-804 (2013).

130. Mosser, D. M. The many faces of macrophage activation. J. Leukoc. Biol. 73, 209-212 (2003).

131. Martinez, F. O. \& Gordon, S. The M1 and M2 paradigm of macrophage activation: time for reassessment. F1000Prime Rep. 6, 13 (2014).

132. Murray, P. J. et al. Macrophage Activation and Polarization: Nomenclature and Experimental Guidelines. Immunity 41, 14-20 (2014).

133. Sica, A. \& Mantovani, A. Macrophage plasticity and polarization: in vivo veritas. J. Clin. Invest. 122, 787-795 (2012).

134. Gordon, S. Alternative activation of macrophages. Nat. Rev. Immunol. 3, 2335 (2003).

135. Kadl, A. et al. Identification of a novel macrophage phenotype that develops in response to atherogenic phospholipids via Nrf2. Circ. Res. 107, 737-46 (2010).

136. Rőszer, T. Understanding the Mysterious M2 Macrophage through Activation Markers and Effector Mechanisms. Mediators Inflamm. 2015, 816460 (2015).

137. Spiller, K. L. et al. The role of macrophage phenotype in vascularization of tissue engineering scaffolds. Biomaterials 35, 4477-4488 (2014).

138. Awojoodu, A. O. et al. Sphingosine 1-phosphate receptor 3 regulates recruitment of anti-inflammatory monocytes to microvessels during implant arteriogenesis. Proc. Natl. Acad. Sci. U. S. A. 110, 13785-90 (2013). 
139. Takeda, Y. et al. Macrophage skewing by Phd2 haplodeficiency prevents ischaemia by inducing arteriogenesis. Nature 479, 122-6 (2011).

140. Fantin, A. et al. Tissue macrophages act as cellular chaperones for vascular anastomosis downstream of VEGF-mediated endothelial tip cell induction. Blood 116, 829-840 (2010).

141. Atashroo, D. A. et al. Assessment of Viability of Human Fat Injection into Nude Mice with Micro-Computed Tomography. J. Vis. Exp. (2015). doi:10.3791/52217

142. Phipps, K. D. et al. Alternatively Activated M2 Macrophages Improve Autologous Fat Graft Survival in a Mouse Model through Induction of Angiogenesis. Plast. Reconstr. Surg. 135, 140-149 (2015).

143. Lumeng, C. N., Bodzin, J. L. \& Saltiel, A. R. Obesity induces a phenotypic switch in adipose tissue macrophage polarization. J. Clin. Invest. 117, 175-84 (2007).

144. Mendel, T. a et al. Pericytes derived from adipose-derived stem cells protect against retinal vasculopathy. PLoS One 8, e65691 (2013).

145. Tabit, C. J., Slack, G. C., Fan, K., Wan, D. C. \& Bradley, J. P. Fat grafting versus adipose-derived stem cell therapy: distinguishing indications, techniques, and outcomes. Aesthetic Plast. Surg. 36, 704-13 (2012).

146. Egami, K. Ischemia-induced angiogenesis: role of inflammatory response mediated by P-selectin. J. Leukoc. Biol. 79, 971-976 (2006).

147. Suga, H. et al. Adipose tissue remodeling under ischemia: death of adipocytes and activation of stem/progenitor cells. Plast. Reconstr. Surg. 126, 1911-23 (2010).

148. Xiong, Y., Mahmood, A. \& Chopp, M. Angiogenesis, neurogenesis and brain recovery of function following injury. Curr. Opin. Investig. Drugs 11, 298-308 (2010).

149. Helisch, A. \& Schaper, W. Arteriogenesis: the development and growth of collateral arteries. Microcirculation 10, 83-97 (2003).

150. Schaper, W. Collateral circulation: past and present. Basic Res. Cardiol. 104, 5-21 (2009).

151. Mac Gabhann, F. \& Peirce, S. M. Collateral Capillary Arterialization following arteriolar ligation in murine skeletal muscle. Microcirculation 17, 333-347 (2010).

152. Bloor, C. M. Angiogenesis during exercise and training. Angiogenesis 8, 263271 (2005).

153. Demir, R., Yaba, A. \& Huppertz, B. Vasculogenesis and angiogenesis in the endometrium during menstrual cycle and implantation. Acta Histochemica 112, 203-214 (2010). 
154. Rogers, P. A. W., Donoghue, J. F., Walter, L. M. \& Girling, J. E. Endometrial angiogenesis, vascular maturation, and lymphangiogenesis. Reprod. Sci. 16, 147-151 (2009).

155. Myers, B. \& Cherry, G. Causes of necrosis in pedicle flaps. Plast. Reconstr. Surg. 42, 43-50 (1968).

156. Kerrigan, C. Skin flap failure: pathophysiology. Plast. Reconstr. Surg. 72, 766-774 (1983).

157. Aydin, M. A. \& Mavili, M. E. Examining microcirculation improves the angiosome theory in explaining the delay phenomenon in a rabbit model. $J$. Reconstr. Microsurg. 19, 187-194 (2003).

158. Cederna, P. S., Chang, P., Pittet-Cuenod, B. M., Razaboni, R. M. \& Cram, A. E. The effect of the delay phenomenon on the vascularity of rabbit rectus abdominis muscles. Plast. Reconstr. Surg. 99, 194-205 (1997).

159. Rasband, W. S. ImageJ. at <http://rsb.info.nih.gov/ij/>

160. Seaman, M. E., Peirce, S. M. \& Kelly, K. Rapid analysis of vessel elements (RAVE): a tool for studying physiologic, pathologic and tumor angiogenesis. PLoS One 6, e20807 (2011).

161. Gealekman, O. et al. Depot-specific differences and insufficient subcutaneous adipose tissue angiogenesis in human obesity. Circulation 123, 186-94 (2011).

162. Jung, S. et al. Analysis of Fractalkine Receptor CX3CR1 Function by Targeted Deletion and Green Fluorescent Protein Reporter Gene Insertion. Mol. Cell. Biol. 20, 4106-4114 (2000).

163. Heil, M. et al. Blood monocyte concentration is critical for enhancement of collateral artery growth. Am. J. Physiol. Heart Circ. Physiol. 283, H2411H2419 (2002).

164. Schaper, J., König, R., Franz, D. \& Schaper, W. The endothelial surface of growing coronary collateral arteries. Intimal margination and diapedesis of monocytes - A combined SEM and TEM study. Virchows Arch. A Pathol. Anat. Histol. 370, 193-205 (1976).

165. Murray, P. J. \& Wynn, T. A. Protective and pathogenic functions of macrophage subsets. Nat. Rev. Immunol. 11, 723-737 (2011).

166. Stein, M., Keshav, S., Harris, N. \& Gordon, S. Interleukin 4 potently enhances murine macrophage mannose receptor activity: a marker of alternative immunologic macrophage activation. J. Exp. Med. 176, 287-292 (1992).

167. Willenborg, S. et al. CCR2 recruits an inflammatory macrophage subpopulation critical for angiogenesis in tissue repair. Blood 120, 613-25 (2012).

168. Porcheray, F. et al. Macrophage activation switching: an asset for the resolution of inflammation. Clin. Exp. Immunol. 0, 051006055454001 (2005). 
169. Gruionu, G., Hoying, J. B., Pries, A. R. \& Secomb, T. W. Structural Remodeling of the Mouse Gracilis Artery: Coordinated Changes in Diameter and Medial Area Maintain Circumferential Stress. Microcirculation 19, 610618 (2012).

170. Hecht, N. et al. Cerebral hemodynamic reserve and vascular remodeling in C57/BL6 mice are influenced by age. Stroke 43, 3052-3062 (2012).

171. Troidl, K. \& Schaper, W. Arteriogenesis versus angiogenesis in peripheral artery disease. Diabetes. Metab. Res. Rev. 28, 27-29 (2012).

172. Ziegler, M. et al. Marvels, Mysteries, and Misconceptions of Vascular Compensation to Peripheral Artery Occlusion. Microcirculation 17, 3-20 (2010).

173. Shireman, P. K. The chemokine system in arteriogenesis and hind limb ischemia. J. Vasc. Surg. 45, 48-56 (2007).

174. Chilian, W. M. et al. Microvascular occlusions promote coronary collateral growth. Am. J. Physiol. 258, H1103-H1111 (1990).

175. Wang, Z. et al. Specific metabolic rates of major organs and tissues across adulthood: Evaluation by mechanistic model of resting energy expenditure. Am. J. Clin. Nutr. 92, 1369-1377 (2010).

176. Marti, H. H. \& Risaut, W. Angiogenesis in ischemic disease. in Thrombosis and Haemostasis 82, 44-52 (1999).

177. Leblanc, A. J., Krishnan, L., Sullivan, C. J., Williams, S. K. \& Hoying, J. B. Microvascular Repair: Post-Angiogenesis Vascular Dynamics. Microcirculation 19, (2012).

178. Ozgentaş, H. E., Shenaq, S. \& Spira, M. Study of the delay phenomenon in the rat TRAM flap model. Plast. Reconstr. Surg. 94, 1018-1024; discussion 10251026 (1994).

179. Trayhurn, P. Hypoxia and Adipose Tissue Function and Dysfunction in Obesity. Physiol. Rev. 93, 1-21 (2013).

180. Seok, J. et al. Genomic responses in mouse models poorly mimic human inflammatory diseases. Proc. Natl. Acad. Sci. U. S. A. 110, 3507-12 (2013).

181. Seaman, S. A., Cao, Y., Campbell, C. A. \& Peirce, S. M. Macrophage Recruitment and Polarization During Collateral Vessel Remodeling in Murine Adipose Tissue. Microcirculation 75-87 (2016). doi:10.1111/micc.12261

182. Zhang, X., Goncalves, R. \& Mosser, D. M. in Current Protocols in Immunology (John Wiley \& Sons, Inc., 2008). doi:10.1002/0471142735.im1401s83

183. Weischenfeldt, J. \& Porse, B. Bone Marrow-Derived Macrophages (BMM): Isolation and Applications. Cold Spring Harb. Protoc. 2008, pdb.prot5080pdb.prot5080 (2008). 
184. Vogel, D. Y. S. et al. Human macrophage polarization in vitro: Maturation and activation methods compared. Immunobiology 219, 695-703 (2014).

185. Weisser, S. B., McLarren, K. W., Kuroda, E. \& Sly, L. M. Generation and characterization of murine alternatively activated macrophages. Methods Mol. Biol. 946, 225-39 (2013).

186. Oh, D. Y., Morinaga, H., Talukdar, S., Bae, E. J. \& Olefsky, J. M. Increased macrophage migration into adipose tissue in obese mice. Diabetes 61, 346-54 (2012).

187. Leor, J. et al. Ex vivo activated human macrophages improve healing, remodeling, and function of the infarcted heart. Circulation 114, 94-101 (2006).

188. Hirose, N. et al. The Local Injection of Peritoneal Macrophages Induces Neovascularization in Rat Ischemic Hind Limb Muscles. Cell Transplant. 17, 211-222 (2008).

189. Jetten, N. et al. Anti-inflammatory M2, but not pro-inflammatory M1 macrophages promote angiogenesis in vivo. Angiogenesis 17, 109-18 (2014).

190. Spiegel, S. \& Milstien, S. Sphingosine-1-phosphate: an enigmatic signalling lipid. Nat. Rev. Mol. Cell Biol. 4, 397-407 (2003).

191. Yatomi, Y. et al. Sphingosine 1-phosphate, a bioactive sphingolipid abundantly stored in platelets, is a normal constituent of human plasma and serum. J. Biochem. 121, 969-73 (1997).

192. Garcia, J. G. et al. Sphingosine 1-phosphate promotes endothelial cell barrier integrity by Edg-dependent cytoskeletal rearrangement. J. Clin. Invest. 108, 689-701 (2001).

193. Liu, Y. et al. Edg-1, the G protein-coupled receptor for sphingosine-1phosphate, is essential for vascular maturation. J. Clin. Invest. 106, 951-61 (2000).

194. Lee, M. et al. Vascular Endothelial Cell Adherens Junction Assembly and Morphogenesis Induced by Sphingosine-1-Phosphate. Cell 99, 301-312 (1999).

195. Sefcik, L. S. et al. Selective activation of sphingosine 1-phosphate receptors 1 and 3 promotes local microvascular network growth. Tissue Eng. Part A 17, $617-29$ (2011).

196. Kupperman, E., An, S., Osborne, N., Waldron, S. \& Stainier, D. Y. A sphingosine-1-phosphate receptor regulates cell migration during vertebrate heart development. Nature 406, 192-5 (2000).

197. Brinkmann, V. et al. The immune modulator FTY720 targets sphingosine 1phosphate receptors. J. Biol. Chem. 277, 21453-7 (2002).

198. Mandala, S. et al. Alteration of lymphocyte trafficking by sphingosine-1phosphate receptor agonists. Science 296, 346-9 (2002). 
199. Graeler, M., Shankar, G. \& Goetzl, E. J. Cutting Edge: Suppression of T Cell Chemotaxis by Sphingosine 1-Phosphate. J. Immunol. 169, 4084-4087 (2002).

200. Brinkmann, V. et al. Fingolimod (FTY720): discovery and development of an oral drug to treat multiple sclerosis. Nat. Rev. Drug Discov. 9, 883-897 (2010).

201. Van Rooijen, N. \& Sanders, A. Liposome mediated depletion of macrophages: mechanism of action, preparation of liposomes and applications. J. Immunol. Methods 174, 83-93 (1994).

202. Van Rooijen, N., Sanders, A. \& Van Den Berg, T. K. Apoptosis of macrophages induced by liposome-mediated intracellular delivery of clodronate and propamidine. J. Immunol. Methods 193, 93-99 (1996).

203. Summan, M. et al. Macrophages and skeletal muscle regeneration: a clodronate-containing liposome depletion study. Am. J. Physiol. Regul. Integr. Comp. Physiol. 290, R1488-R1495 (2006).

204. Hays, P. L. et al. The role of macrophages in early healing of a tendon graft in a bone tunnel. J. Bone Joint Surg. Am. 90, 565-579 (2008).

205. Ferenbach, D. A. et al. Macrophage/monocyte depletion by clodronate, but not diphtheria toxin, improves renal ischemia/reperfusion injury in mice. Kidney International 82, 928-933 (2012).

206. Staffend, N. A. \& Meisel, R. L. DiOlistic Labeling of Neurons in Tissue Slices: A Qualitative and Quantitative Analysis of Methodological Variations. Front. Neuroanat. 5, (2011).

207. Lumeng, C. N., DelProposto, J. B., Westcott, D. J. \& Saltiel, A. R. Phenotypic Switching of Adipose Tissue Macrophages With Obesity Is Generated by Spatiotemporal Differences in Macrophage Subtypes. Diabetes 57, 3239-3246 (2008).

208. Denholm, E. M. \& Stankus, G. P. Differential effects of two fluorescent probes on macrophage migration as assessed by manual and automated methods. Cytometry 19, 366-9 (1995).

209. Huang, C. et al. Local delivery of FTY720 accelerates cranial allograft incorporation and bone formation. Cell Tissue Res. 347, 553-566 (2012).

210. Sefcik, L. S., Petrie Aronin, C. E., Wieghaus, K. A. \& Botchwey, E. A. Sustained release of sphingosine 1-phosphate for therapeutic arteriogenesis and bone tissue engineering. Biomaterials 29, 2869-2877 (2008).

211. Kurashima, Y. et al. Sphingosine 1-Phosphate-Mediated Trafficking of Pathogenic Th2 and Mast Cells for the Control of Food Allergy. J. Immunol. 179, 1577-1585 (2007).

212. Frink, M. et al. FTY720 improves survival after transient ischemia and reperfusion of the hind limbs. J. Trauma 63, 263-7 (2007). 
213. Gorlino, C. V et al. Neutrophils exhibit differential requirements for homing molecules in their lymphatic and blood trafficking into draining lymph nodes. J. Immunol. 193, 1966-74 (2014).

214. Danenberg, H. D. Macrophage Depletion by Clodronate-Containing Liposomes Reduces Neointimal Formation After Balloon Injury in Rats and Rabbits. Circulation 106, 599-605 (2002).

215. Ohki, Y. et al. Granulocyte colony-stimulating factor promotes neovascularization by releasing vascular endothelial growth factor from neutrophils. FASEB J. 19, 2005-7 (2005).

216. van Rooijen, N., Kors, N. \& Kraal, G. Macrophage subset repopulation in the spleen: differential kinetics after liposome-mediated elimination. J. Leukoc. Biol. 45, 97-104 (1989).

217. Biewenga, J. et al. Macrophage depletion in the rat after intraperitoneal administration of liposome-encapsulated clodronate: depletion kinetics and accelerated repopulation of peritoneal and omental macrophages by administration of Freund's adjuvant. Cell Tissue Res. 280, 189-96 (1995).

218. Xie, Y. et al. An integrated fat grafting technique for cosmetic facial contouring. J. Plast. Reconstr. Aesthet. Surg. 63, 270-6 (2010).

219. Xie, Y., Li, Q., Zheng, D., Lei, H. \& Pu, L. L. Q. Correction of hemifacial atrophy with autologous fat transplantation. Ann. Plast. Surg. 59, 645-53 (2007).

220. Yang, H. \& Lee, H. Successful use of squeezed-fat grafts to correct a breast affected by Poland syndrome. Aesthetic Plast. Surg. 35, 418-25 (2011).

221. Spear, S. L., Wilson, H. B. \& Lockwood, M. D. Fat Injection to Correct Contour Deformities in the Reconstructed Breast. Plast. Reconstr. Surg. 116, 13001305 (2005).

222. Lee, J. H. et al. The effect of pressure and shear on autologous fat grafting. Plastic and reconstructive surgery 131, (2013).

223. Li, J. et al. Supplementing fat grafts with adipose stromal cells for cosmetic facial contouring. Dermatol. Surg. 39, 449-56 (2013).

224. Coleman, S. R. Structural fat grafts: the ideal filler? Clin. Plast. Surg. 28, 111-9 (2001).

225. Eto, H. et al. Characterization of structure and cellular components of aspirated and excised adipose tissue. Plast. Reconstr. Surg. 124, 1087-97 (2009).

226. Gentile, P. et al. Adipose-derived stromal vascular fraction cells and plateletrich plasma: basic and clinical evaluation for cell-based therapies in patients with scars on the face. J. Craniofac. Surg. 25, 267-72 (2014). 
227. Chang, Q., Li, J., Dong, Z., Liu, L. \& Lu, F. Quantitative volumetric analysis of progressive hemifacial atrophy corrected using stromal vascular fractionsupplemented autologous fat grafts. Dermatol. Surg. 39, 1465-73 (2013).

228. Gir, P., Oni, G., Brown, S. a, Mojallal, A. \& Rohrich, R. J. Human adipose stem cells: current clinical applications. Plast. Reconstr. Surg. 129, 1277-90 (2012).

229. Janke, J., Engeli, S., Gorzelniak, K., Luft, F. C. \& Sharma, A. M. Mature Adipocytes Inhibit In Vitro Differentiation of Human Preadipocytes via Angiotensin Type 1 Receptors. Diabetes 51, 1699-1707 (2002).

230. Stacey, D. H., Hanson, S. E., Lahvis, G., Gutowski, K. a \& Masters, K. S. In vitro adipogenic differentiation of preadipocytes varies with differentiation stimulus, culture dimensionality, and scaffold composition. Tissue Eng. Part A 15, 3389-99 (2009).

231. Lamers, D. et al. Dipeptidyl peptidase 4 is a novel adipokine potentially linking obesity to the metabolic syndrome. Diabetes 60, 1917-25 (2011).

232. Fain, J. N., Madan, A. K., Hiler, M. L., Cheema, P. \& Bahouth, S. W. Comparison of the release of adipokines by adipose tissue, adipose tissue matrix, and adipocytes from visceral and subcutaneous abdominal adipose tissues of obese humans. Endocrinology 145, 2273-82 (2004).

233. Goralski, K. B. et al. Chemerin, a novel adipokine that regulates adipogenesis and adipocyte metabolism. J. Biol. Chem. 282, 28175-88 (2007).

234. Nguyen, M. T. A. et al. A subpopulation of macrophages infiltrates hypertrophic adipose tissue and is activated by free fatty acids via Toll-like receptors 2 and 4 and JNK-dependent pathways. J. Biol. Chem. 282, 3527992 (2007).

235. Chang, H. et al. Safety of adipose-derived stem cells and collagenase in fat tissue preparation. Aesthetic Plast. Surg. 37, 802-8 (2013).

236. Adipose Tissue and Adipokines in Health and Disease. (Humana Press, 2007).

237. de Benito, J., Fernández, I. \& Nanda, V. Treatment of depressed scars with a dissecting cannula and an autologous fat graft. Aesthetic Plast. Surg. 23, 36770 (1999).

238. Eto, H. et al. The fate of adipocytes after nonvascularized fat grafting: evidence of early death and replacement of adipocytes. Plast. Reconstr. Surg. 129, 1081-92 (2012).

239. Piasecki, J. H., Gutowski, K. a, Lahvis, G. P. \& Moreno, K. I. An experimental model for improving fat graft viability and purity. Plast. Reconstr. Surg. 119, 1571-83 (2007).

240. Williams, S. K., McKenney, S. \& Jarrell, B. E. Collagenase lot selection and purification for adipose tissue digestion. Cell Transplant. 4, 281-9 
241. Gilpin, D. et al. Injectable collagenase Clostridium histolyticum: a new nonsurgical treatment for Dupuytren's disease. J. Hand Surg. Am. 35, 202738.e1 (2010).

242. Honig, S. C. Intralesional collagenase in the treatment of Peyronie's disease. Ther. Adv. Urol. 6, 47-53 (2014).

243. Gutowski, K. a. Current applications and safety of autologous fat grafts: a report of the ASPS fat graft task force. Plast. Reconstr. Surg. 124, 272-80 (2009).

244. Suga, H. et al. Numerical measurement of viable and nonviable adipocytes and other cellular components in aspirated fat tissue. Plast. Reconstr. Surg. 122, 103-14 (2008).

245. Gantenbein-Ritter, B., Sprecher, C. M., Chan, S., Illien-Jünger, S. \& Grad, S. Confocal imaging protocols for live/dead staining in three-dimensional carriers. Methods Mol. Biol. 740, 127-40 (2011).

246. Eto, H. et al. Adipose injury-associated factors mitigate hypoxia in ischemic tissues through activation of adipose-derived stem/progenitor/stromal cells and induction of angiogenesis. Am. J. Pathol. 178, 2322-32 (2011).

247. Surgery, T. A. S. for A. P. 2013 Cosmetic Surgery National Data Bank Statistics. (2013).

248. Aksu, a E., Rubin, J. P., Dudas, J. R. \& Marra, K. G. Role of gender and anatomical region on induction of osteogenic differentiation of human adiposederived stem cells. Ann. Plast. Surg. 60, 306-22 (2008).

249. Ogawa, R. et al. Adipogenic differentiation by adipose-derived stem cells harvested from GFP transgenic mice-including relationship of sex differences. Biochem. Biophys. Res. Commun. 319, 511-7 (2004).

250. Gir, P. et al. Fat grafting: evidence-based review on autologous fat harvesting, processing, reinjection, and storage. Plast. Reconstr. Surg. 130, 249-58 (2012).

251. Agha, R. A., Goodacre, T. \& Orgill, D. P. Use of autologous fat grafting for reconstruction postmastectomy and breast conserving surgery: a systematic review protocol. BMJ Open 3, e003709-e003709 (2013).

252. Topcu, A., Aydin, O. E., Ünlü, M., Barutcu, A. \& Atabey, A. Increasing the viability of fat grafts by vascular endothelial growth factor. Arch. Facial Plast. Surg. 14, 270-6

253. Sommer, B. \& Sattler, G. Current concepts of fat graft survival: histology of aspirated adipose tissue and review of the literature. Dermatologic Surg. 26, 1159-66 (2000).

254. Paik, K. J. et al. Studies in Fat Grafting: Part V. Cell-Assisted Lipotransfer to Enhance Fat Graft Retention Is Dose Dependent. Plast. Reconstr. Surg. 136, 67-75 (2015). 
255. Garza, R. M. et al. Studies in fat grafting: Part IV. Adipose-derived stromal cell gene expression in cell-assisted lipotransfer. Plast. Reconstr. Surg. 135, 1045-55 (2015).

256. Fu, S. et al. Fate of Adipose-Derived Stromal Vascular Fraction Cells after Coimplantation with Fat Grafts. Plast. Reconstr. Surg. 132, 363-373 (2013).

257. Aronowitz, J. A., Lockhart, R. A. \& Hakakian, C. S. Mechanical versus enzymatic isolation of stromal vascular fraction cells from adipose tissue. Springerplus 4, 713 (2015).

258. Agrawal, H. et al. Human adipose-derived stromal/stem cells demonstrate short-lived persistence after implantation in both an immunocompetent and an immunocompromised murine model. Stem Cell Res. Ther. 5, 142 (2014).

259. Gentile, P. et al. Adipose-Derived Stromal Vascular Fraction Cells and Platelet-Rich Plasma. J. Craniofac. Surg. 25, 267-272 (2014).

260. Youn, S., Shin, J. I., Kim, J. Do, Kim, J. T. \& Kim, Y. H. Correction of infraorbital dark circles using collagenase-digested fat cell grafts. Dermatol. Surg. 39, 766-72 (2013).

261. Gilpin, D. et al. Injectable Collagenase Clostridium Histolyticum: A New Nonsurgical Treatment for Dupuytren's Disease. J. Hand Surg. Am. 35, 20272038.e1 (2010).

262. Honig, S. C. Intralesional collagenase in the treatment of Peyronie's disease. Ther. Adv. Urol. 6, 47-53 (2014).

263. Seaman, S. A., Tannan, S. C., Cao, Y., Peirce, S. M. \& Lin, K. Y. Differential Effects of Processing Time and Duration of Collagenase Digestion on Human and Murine Fat Grafts. Plast. Reconstr. Surg. 136, 189e-199e (2015).

264. Atik, B., Ozturk, G., Erdogan, E. \& Tan, O. Comparison of Techniques for Long-Term Storage of Fat Grafts: An Experimental Study. Plast. Reconstr. Surg. 118, 1533-1537 (2006).

265. Martin, A. D., Daniel, M. Z., Drinkwater, D. T. \& Clarys, J. P. Adipose tissue density, estimated adipose lipid fraction and whole body adiposity in male cadavers. Int. J. Obes. Metab. Disord. 18, 79-83 (1994).

266. Smith, P. et al. Autologous human fat grafting: effect of harvesting and preparation techniques on adipocyte graft survival. Plast. Reconstr. Surg. 117, 1836-44 (2006).

267. Ruan, H., Zarnowski, M. J., Cushman, S. W. \& Lodish, H. F. Standard Isolation of Primary Adipose Cells from Mouse Epididymal Fat Pads Induces Inflammatory Mediators and Down-regulates Adipocyte Genes. J. Biol. Chem. 278, 47585-47593 (2003).

268. Thompson, A. C. S. et al. Mitigation of isolation-associated adipocyte interleukin-6 secretion following rapid dissociation of adipose tissue. J. Lipid Res. 53, 2797-2805 (2012). 
269. Makki, K., Froguel, P. \& Wolowczuk, I. Adipose Tissue in Obesity-Related Inflammation and Insulin Resistance: Cells, Cytokines, and Chemokines. ISRN Inflamm. 2013, 1-12 (2013).

270. Shi, C. et al. IL-6 and TNF- $\alpha$ Induced Obesity-Related Inflammatory Response Through Transcriptional Regulation of miR-146b. J. Interf. Cytokine Res. 34, 342-348 (2014).

271. Coppack, S. W. Pro-inflammatory cytokines and adipose tissue. Proc. Nutr. Soc. 60, 349-56 (2001).

272. Gentile, P. et al. A Comparative Translational Study: The Combined Use of Enhanced Stromal Vascular Fraction and Platelet-Rich Plasma Improves Fat Grafting Maintenance in Breast Reconstruction. Stem Cells Transl. Med. 1, 341-351 (2012).

273. Shultz, L. D., Ishikawa, F. \& Greiner, D. L. Humanized mice in translational biomedical research. Nat. Rev. Immunol. 7, 118-130 (2007).

274. Christianson, S. W. et al. Enhanced human CD4+ T cell engraftment in beta2microglobulin-deficient NOD-scid mice. J. Immunol. 158, 3578-86 (1997).

275. Avram, M. M. \& Harry, R. S. Cryolipolysis for subcutaneous fat layer reduction. Lasers Surg. Med. 41, 703-8 (2009).

276. Stevens, W. G., Pietrzak, L. K. \& Spring, M. a. Broad overview of a clinical and commercial experience with CoolSculpting. Aesthet. Surg. J. 33, 835-46 (2013).

277. Manstein, D. et al. Selective cryolysis: A novel method of non-invasive fat removal. Lasers Surg. Med. 40, 595-604 (2008).

278. Coleman, S. R., Sachdeva, K., Egbert, B. M., Preciado, J. \& Allison, J. Clinical efficacy of noninvasive cryolipolysis and its effects on peripheral nerves. Aesthetic Plast. Surg. 33, 482-488 (2009).

279. Sasaki, G. H., Abelev, N. \& Tevez-Ortiz, A. Noninvasive selective cryolipolysis and reperfusion recovery for localized natural fat reduction and contouring. Am. Soc. Aesthetic Plast. Surg. 34, 420-31 (2014).

280. Jalian H, Avram MM, Garibyan L, Mihm MC \& Anderson R. Paradoxical adipose hyperplasia after cryolipolysis. JAMA Dermatology 150, 317-319 (2014).

281. Nelson, A. A., Wasserman, D. \& Avram, M. M. Cryolipolysis for reduction of excess adipose tissue. Semin. Cutan. Med. Surg. 28, 244-9 (2009).

282. American Society of Aesthetic Plastic Surgeons. 2014 Cosmetic Surgery National Data Bank Statistics. doi:http://dx.doi.org/10.1177/1090820X13493905

283. Derrick, C., Shridharani, S. \& Broyles, J. The Safety and Efficacy of Cryolipolysis: A Systematic Review of Available Literature. Aesthetic Surg. J. (2015). 
284. Poon, I. K. H., Lucas, C. D., Rossi, A. G. \& Ravichandran, K. S. Apoptotic cell clearance: basic biology and therapeutic potential. Nat. Rev. Immunol. 14, 166-80 (2014).

285. Mathew M. Avram \& Rosemary S. Harry. Cryolipolysis(TM) for Subcutaneous Fat Layer Reduction. Lasers Surg. Med. 41703-708 (2009

286. Jalian, H. R. \& Avram, M. M. Body contouring: the skinny on noninvasive fat removal. Semin. Cutan. Med. Surg. 31, 121-5 (2012).

287. Stefani, W. Adipose Hypertrophy Following Cryolipolysis. Aesthetic Surg. J. (2015).

288. Halberg, N. et al. Hypoxia-inducible factor 1alpha induces fibrosis and insulin resistance in white adipose tissue. Mol. Cell. Biol. 29, 4467-83 (2009).

289. Norman, J. T., Clark, I. M. \& Garcia, P. L. Hypoxia promotes fibrogenesis in human renal fibroblasts. Kidney Int. 58, 2351-66 (2000).

290. Watson, C. J. et al. Hypoxia-induced epigenetic modifications are associated with cardiac tissue fibrosis and the development of a myofibroblast-like phenotype. Hum. Mol. Genet. 23, 2176-88 (2014).

291. Wound Care Markets, Kalorama Information, Skin Ulcers I. (2005).

292. Kroll, S. S., Reece, G. P., Miller, M. J. \& Schusterman, M. A. Comparison of the rectus abdominis free flap with the pectoralis major myocutaneous flap for reconstructions in the head and neck. Am. J. Surg. 164, 615-8 (1992).

293. Shrotria, S., Webster, D. J., Mansel, R. E. \& Hughes, L. E. Complications of rectus abdominis myocutaneous flaps in breast surgery. Eur. J. Surg. Oncol. 19, 80-3 (1993).

294. Zhang, F. et al. Improvement of skin paddle survival by application of vascular endothelial growth factor in a rat TRAM flap model. Ann. Plast. Surg. 46, 314-9 (2001).

295. Roussakis, E., Li, Z., Nichols, A. J. \& Evans, C. L. Oxygen-Sensing Methods in Biomedicine from the Macroscale to the Microscale. Angew. Chemie Int. Ed. 54, 8340-8362 (2015).

296. Schreml, S. et al. Oxygen in acute and chronic wound healing. Br. J. Dermatol. 163, 257-268 (2010).

297. Kalani, M., Brismar, K., Fagrell, B., Ostergren, J. \& Jorneskog, G. Transcutaneous oxygen tension and toe blood pressure as predictors for outcome of diabetic foot ulcers. Diabetes Care 22, 147-151 (1999).

298. Yudovsky, D., Nouvong, A. \& Pilon, L. Hyperspectral imaging in diabetic foot wound care. J. Diabetes Sci. Technol. 4, 1099-113 (2010).

299. Wolfbeis, O. S. Luminescent sensing and imaging of oxygen: Fierce competition to the Clark electrode. BioEssays 37, 921-928 (2015). 
300. Wang, X. \& Wolfbeis, O. S. Optical methods for sensing and imaging oxygen: materials, spectroscopies and applications. Chem. Soc. Rev. 43, 3666-3761 (2014).

301. Nichols, A. J., Roussakis, E., Klein, O. J. \& Evans, C. L. Click-Assembled, Oxygen-Sensing Nanoconjugates for Depth-Resolved, Near-Infrared Imaging in a 3 D Cancer Model. Angew. Chemie Int. Ed. 53, 3671-3674 (2014).

302. Lemon, C. M. et al. Micelle-Encapsulated Quantum Dot-Porphyrin Assemblies as in Vivo Two-Photon Oxygen Sensors. J. Am. Chem. Soc. 137, 9832-9842 (2015).

303. Lebedev, A. Y. et al. Dendritic Phosphorescent Probes for Oxygen Imaging in Biological Systems. ACS Appl. Mater. Interfaces 1, 1292-1304 (2009).

304. Zheng, X. et al. Hypoxia-specific ultrasensitive detection of tumours and cancer cells in vivo. Nat. Commun. 6, 5834 (2015).

305. Hara, D., Komatsu, H., Son, A., Nishimoto, S. \& Tanabe, K. Water-Soluble Phosphorescent Ruthenium Complex with a Fluorescent Coumarin Unit for Ratiometric Sensing of Oxygen Levels in Living Cells. Bioconjug. Chem. 26, 645-649 (2015).

306. Yoshihara, T., Yamaguchi, Y., Hosaka, M., Takeuchi, T. \& Tobita, S. Ratiometric Molecular Sensor for Monitoring Oxygen Levels in Living Cells. Angew. Chemie 124, 4224-4227 (2012).

307. Zhao, Q. et al. Fluorescent/phosphorescent dual-emissive conjugated polymer dots for hypoxia bioimaging. Chem. Sci. 6, 1825-1831 (2015).

308. Wu, C., Bull, B., Christensen, K. \& McNeill, J. Ratiometric SingleNanoparticle Oxygen Sensors for Biological Imaging. Angew. Chemie Int. Ed. 48, 2741-2745 (2009).

309. Dmitriev, R. I. et al. Versatile Conjugated Polymer Nanoparticles for HighResolution O 2 Imaging in Cells and 3D Tissue Models. ACS Nano 9, 52755288 (2015).

310. McLaurin, E. J., Greytak, A. B., Bawendi, M. G. \& Nocera, D. G. Two-Photon Absorbing Nanocrystal Sensors for Ratiometric Detection of Oxygen. J. Am. Chem. Soc. 131, 12994-13001 (2009).

311. Zhang, G., Lu, J., Sabat, M. \& Fraser, C. L. Polymorphism and Reversible Mechanochromic Luminescence for Solid-State Difluoroboron Avobenzone. J. Am. Chem. Soc. 132, 2160-2162 (2010).

312. Wang, L. et al. Luminescent Chromism of Boron Diketonate Crystals: Distinct Responses to Different Stresses. Adv. Mater. 27, 2918-2922 (2015).

313. Poon, C.-T., Wu, D., Lam, W. H. \& Yam, V. W.-W. A Solution-Processable Donor-Acceptor Compound Containing Boron(III) Centers for Small-MoleculeBased High-Performance Ternary Electronic Memory Devices. Angew. Chemie 127, 10715-10719 (2015). 
314. Koch, M. et al. Metal-Free Triplet Phosphors with High Emission Efficiency and High Tunability. Angew. Chemie Int. Ed. 53, 6378-6382 (2014).

315. Cogné-Laage, E. et al. Diaroyl(methanato)boron difluoride compounds as medium-sensitive two-photon fluorescent probes. Chemistry (Easton). 10, $1445-55$ (2004).

316. Zhang, X. et al. Design and Synthesis of Curcumin Analogues for in Vivo Fluorescence Imaging and Inhibiting Copper-Induced Cross-Linking of Amyloid Beta Species in Alzheimer's Disease. J. Am. Chem. Soc. 135, 1639716409 (2013).

317. Zhang, G. et al. Multi-Emissive Difluoroboron Dibenzoylmethane Polylactide Exhibiting Intense Fluorescence and Oxygen-Sensitive Room-Temperature Phosphorescence. J. Am. Chem. Soc. 129, 8942-8943 (2007).

318. Zhang, X. et al. General Design Strategy for Aromatic Ketone-Based SingleComponent Dual-Emissive Materials. ACS Appl. Mater. Interfaces 6, 22792284 (2014).

319. Lehner, P., Staudinger, C., Borisov, S. M. \& Klimant, I. Ultra-sensitive optical oxygen sensors for characterization of nearly anoxic systems. Nat. Commun. $\mathbf{5},(2014)$.

320. Zhang, G., Palmer, G. M., Dewhirst, M. W. \& Fraser, C. L. A dual-emissivematerials design concept enables tumour hypoxia imaging. Nat. Mater. 8, $747-751$ (2009).

321. Bowers, D. T. et al. Spatiotemporal Oxygen Sensing Using Dual Emissive Boron Dye-Polylactide Nanofibers. ACS Nano 8, 12080-12091 (2014).

322. Pfister, A., Zhang, G., Zareno, J., Horwitz, A. F. \& Fraser, C. L. Boron Polylactide Nanoparticles Exhibiting Fluorescence and Phosphorescence in Aqueous Medium. ACS Nano 2, 1252-1258 (2008).

323. Meier, R. J. et al. Simultaneous Photographing of Oxygen and $\mathrm{pH}$ In Vivo Using Sensor Films. Angew. Chemie Int. Ed. 50, 10893-10896 (2011).

324. Wang, X. et al. Ultra-Small, Highly Stable, and Sensitive Dual Nanosensors for Imaging Intracellular Oxygen and pH in Cytosol. J. Am. Chem. Soc. 134, 17011-17014 (2012).

325. Samonina-Kosicka, J., DeRosa, C. A., Morris, W. A., Fan, Z. \& Fraser, C. L. Dual-Emissive Difluoroboron Naphthyl-Phenyl $\beta$-Diketonate Polylactide Materials: Effects of Heavy Atom Placement and Polymer Molecular Weight. Macromolecules 47, 3736-3746 (2014).

326. Liu, M. et al. 12-hydroxyheptadecatrienoic acid promotes epidermal wound healing by accelerating keratinocyte migration via the BLT2 receptor. J. Exp. Med. 211, 1063-1078 (2014).

327. Schneider, C. a, Rasband, W. S. \& Eliceiri, K. W. NIH Image to ImageJ: 25 years of image analysis. Nat. Methods 9, 671-675 (2012). 
328. Li, Z. et al. Non-invasive transdermal two-dimensional mapping of cutaneous oxygenation with a rapid-drying liquid bandage. Biomed. Opt. Express 5, 3748 (2014).

329. Schreml, S. et al. 2D luminescence imaging of physiological wound oxygenation. Exp. Dermatol. 20, 550-554 (2011).

330. Schreml, S. et al. Luminescent Dual Sensors Reveal Extracellular pHGradients and Hypoxia on Chronic Wounds That Disrupt Epidermal Repair. Theranostics 4, 721-735 (2014).

331. Rasal, R. M., Janorkar, A. V. \& Hirt, D. E. Poly(lactic acid) modifications. Prog. Polym. Sci. 35, 338-356 (2010).

332. Banner, L. W. Venus Envy: A History of Cosmetic Surgery. Bull. Hist. Med. 73, 171-173 (1999).

333. Mojallal, A. \& Foyatier, J.-L. Historical review of the use of adipose tissue transfer in plastic and reconstructive surgery. Ann. Chir. Plast. esthétique 49, 419-25 (2004).

334. Peer, L. A. The neglected free fat graft. Plast. Reconstr. Surg. 18, 233-50 (1956).

335. Sievers, M. et al. Gene expression and immunohistochemistry in adipose tissue of HIV type 1-infected patients with nucleoside analogue reversetranscriptase inhibitor-associated lipoatrophy. J. Infect. Dis. 200, 252-262 (2009).

336. Lin, G. et al. Defining Stem and Progenitor Cells within Adipose Tissue. Stem Cells Dev. 17, 1053-1063 (2008).

337. Hu, S., Maslov, K. \& Wang, L. V. Second-generation optical-resolution photoacoustic microscopy with improved sensitivity and speed. Opt. Lett. 36, 1134-1136 (2011).

338. $\mathrm{Hu}, \mathrm{S}$. \& Wang, L. V. Photoacoustic imaging and characterization of the microvasculature. J. Biomed. Opt. 15, 011101 (2010).

339. Ning, B. et al. Ultrasound-aided Multi-parametric Photoacoustic Microscopy of the Mouse Brain. Sci. Rep. 5, 18775 (2015).

340. Mericli, A. F. et al. Deferoxamine mitigates radiation-induced tissue injury in a rat irradiated TRAM flap model. Plast. Reconstr. Surg. 135, 124e-34e (2015).

341. Cribbs, R. K., Luquette, M. H. \& Besner, G. E. A standardized model of partial thickness scald burns in mice. J. Surg. Res. 80, 69-74 (1998). 